\title{
Multiscale X-Ray Analysis of Biological Cells and Tissues by Scanning Diffraction and Coherent Imaging
}

\author{
Dissertation \\ zur Erlangung des mathematisch-naturwissenschaftlichen \\ Doktorgrades \\ „Doctor rerum naturalium“ \\ der Georg-August-Universität Göttingen \\ im Promotionsprogramm ProPhys \\ der Georg-August University School of Science (GAUSS)
}

vorgelegt von

Jan-David Nicolas

aus Bad Pyrmont

Göttingen, 2018 


\section{Mitglieder des Betreuungsausschusses:}

Prof. Dr. Tim Salditt

Institut für Röntgenphysik,

Georg-August-Universität Göttingen

Prof. Dr. Eberhard Bodenschatz

Institut für Nichtlineare Dynamik,

Georg-August-Universität Göttingen

\section{Mitglieder der Prüfungskommission:}

Referent:

Prof. Dr. Tim Salditt

Institut für Röntgenphysik,

Georg-August-Universität Göttingen

1. Korreferent: $\quad$ Prof. Dr. Eberhard Bodenschatz

Institut für Nichtlineare Dynamik,

Georg-August-Universität Göttingen

\section{Weitere Mitglieder der Prüfungskommission:}

Prof. Dr. Sarah Köster

Institut für Röntgenphysik,

Georg-August-Universität Göttingen

Prof. Dr. Wolfram-Hubertus Zimmermann

Institut für Pharmakologie und Toxikologie,

Universitätsmedizin Göttingen
Dr. Florian Rehfeldt

III. Physikalisches Institut,

Georg-August-Universität Göttingen

PD Dr. Martin Wenderoth

IV. Physikalisches Institut,

Georg-August-Universität Göttingen

\section{Tag der mündlichen Prüfung:}

5. Juli 2018 
"I am, of course, most ignorant about all things biological, but I imagine most X-ray people start that way."

Rosalind Franklin 



\section{Contents}

$\begin{array}{ll}\text { Abstract } & \text { ix }\end{array}$

1. X-ray diffraction and coherent imaging 1

1.1. X-ray diffraction on muscle . . . . . . . . . . . . . . . . . 1

1.2. Adding real space resolution . . . . . . . . . . . . . . . . 6

1.3. Mapping of neuronal tissue . . . . . . . . . . . . . . . . . . 9

1.4. Coherent imaging with hard X-rays: From cells to tissues . . . . . 10

1.5. Future directions . . . . . . . . . . . . . . . . . . 14

2. Combined scanning X-ray diffraction and holographic imaging $\begin{array}{ll}\text { of cardiomyocytes } & \mathbf{1 7}\end{array}$

2.1. Introduction . . . . . . . . . . . . . . . . . . 17

2.2. Materials and methods . . . . . . . . . . . . . . . . 20

2.2.1. Sample preparation . . . . . . . . . . . 20

2.2.2. Experimental parameters and instrumentation . . . . . 21

2.3. Analysis of cellular diffraction patterns . . . . . . . . . . . . . 22

2.4. Holographic imaging . . . . . . . . . . . . . . . . . 27

2.5. Summary, conclusion and outlook . . . . . . . . . . . . . 31

3. Scanning X-ray diffraction on cardiac tissue: automatized data analysis and processing $\quad 33$

3.1. Introduction . . . . . . . . . . . . . . . . 33

3.2. Scanning X-ray diffraction on cardiac tissue . . . . . . . . . . 35

3.2.1. Experiment . . . . . . . . . . . . . . . . 35

3.2.2. Results .................. 37

3.3. Processing and analysis of scanning diffraction data . . . . . . . . 42

3.4. Summary and conclusion . . . . . . . . . . . . . . . 44

4. X-ray diffraction imaging of cardiac cells and tissue $\mathbf{5 1}$

4.1. Introduction . . . . . . . . . . . . . . . . 51

4.2. Different sample preparation states and X-ray optical setups . . . . 54

4.2.1. Freeze-dried cells . . . . . . . . . . . . 54 
4.2.2. Micropatterning . . . . . . . . . . . . . . 58

4.2.3. Cryogenic recordings . . . . . . . . . . . . . . 60

4.2.4. Living cells . . . . . . . . . . . . . 63

4.3. Cardiac tissue . . . . . . . . . . . . . . . . 66

4.4. Adult cardiac muscle cells . . . . . . . . . . . . . . . . . . . 70

4.5. Correlative microscopy . . . . . . . . . . . . . . . . . . . 74

4.6. Summary and synopsis . . . . . . . . . . . . . . . 76

5. Imaging of neuronal tissues by X-ray diffraction and X-ray fluorescence microscopy: evaluation of contrast and biomarkers for $\begin{array}{ll}\text { neurodegenerative diseases } & \mathbf{8 1}\end{array}$

5.1. Introduction . . . . . . . . . . . . . . . . . . . . . . . . 82

5.2. Materials and methods . . . . . . . . . . . . . . 83

5.3. Results...................... 86

5.4. Discussion and outlook . . . . . . . . . . . . . . . . 98

6. The optical stretcher as a tool for single-particle X-ray imaging and diffraction $\quad 103$

6.1. Introduction . . . . . . . . . . . . . . . . . . . . 104

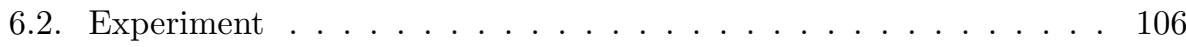

6.2.1. Optical stretcher system . . . . . . . . . . . 106

6.2.2. Integration into the experimental endstation . . . . . . . . 108

6.3. Results . . . . . . . . . . . . . . . . . . . . 112

6.3.1. Polystyrene beads . . . . . . . . . . . . . . . 112

6.3.2. Macrophages loaded with barium sulfate . . . . . . . . . . 114

6.3.3. Three-dimensional iterative reconstruction of a rotating macro-

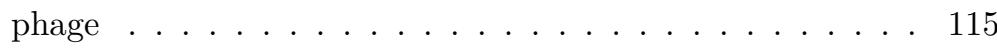

6.4. Summary and conclusion ................. 117

$\begin{array}{ll}\text { 7. Summary and outlook } & 121\end{array}$

$\begin{array}{ll}\text { Appendix } & 125\end{array}$

A. Experimental aspects of scanning X-ray diffraction 127

B. Recommendations on the data analysis of scanning diffraction data 
Authors contribution

Publications

Acknowledgements 165

Curriculum Vitae

169 



\section{Abstract}

The past 70 years of muscle research have profoundly shaped our current understanding of the structure and function of muscle. X-ray diffraction became a key method in its structural analysis and yielded valuable insights into the molecular arrangement of the contraction apparatus. With the availability of synchrotron radiation, structural changes occurring during contraction can now be observed in real time with millisecond time resolution and to date $\mathrm{X}$-ray diffraction is still being used extensively to study muscle contraction in skeletal and cardiac tissue. This work employs an extension of the X-ray diffraction methodology, scanning $\mathrm{X}$-ray diffraction, for structural imaging of biological cells and tissue. By scanning a sample through the focus of an X-ray beam, one can record diffraction patterns at each scan point, and one or multiple features from the diffraction patterns can be analyzed such that maps of the underlying structure can be generated. This approach is also termed diffraction imaging and offers a quantitative and highly selective contrast and a high real space resolution given by the focal spot size of the X-ray beam, which in principle can be well below $100 \mathrm{~nm}$. With recent improvements in X-ray optics, this technique became more easily accessible and has to date been applied to a large variety of sample systems, ranging from hard-condensed matter to biological materials such as wood or bone, however, with very little application for soft tissue. It was the primordial goal of this thesis to fill this gap and streamline the use of scanning X-ray diffraction to image isolated cardiac cells and tissue, even under physiological conditions. We show how such experiments can contribute to the understanding of cardiac tissue architecture and in particular to the development of the contraction apparatus upon cell maturation.

The development of nanofocused X-ray beams has also profited X-ray imaging methods, in particular lensless imaging. As an example for an imaging method that does not require image-forming lenses, we have used in-line holographic imaging as a tool for single cell imaging and combined this technique with scanning X-ray diffraction. Holographic imaging is ideally suited in this respect as it is highly sensitive to phase shifts from the object, produces an image of the object with a resolution typically below $100 \mathrm{~nm}$ and is minimally dose-invasive, in stark contrast to scanning X-ray diffraction. 
The technical details of scanning X-ray diffraction and holographic imaging are introduced in Chapter 1. The first proof-of-concept study that demonstrates the usefulness of combining holographic imaging with scanning X-ray diffraction is then described in Chapter 2. We show that, in combination, the two methods can cover three orders of magnitude in resolution, from approximately $10 \mathrm{~nm}$ to $10 \mu \mathrm{m}$. Furthermore, the impact of radiation damage onto the recorded signal is critically assessed as radiation damage is a key limitation in scanning X-ray diffraction experiments.

In Chapter 3, scanning X-ray diffraction is used to generate diffraction maps with more than one million scan points. The recorded diffraction patterns present a massive data load that had led to the development of a new framework for the analysis and processing of such large amounts of data. Most importantly, for the purpose of increasing the accessibility of scanning X-ray diffraction for a broader community, all tools have been made publicly available.

Technical challenges associated with sample preparation and how the experimental parameters can be optimized is described in greater detail in Chapter 4 . It is also shown what signals can be expected from scanning X-ray diffraction experiments on cardiac cells and tissue and reasonable advice is given on how to unlock the full potential of scanning X-ray diffraction in follow-up experiments.

In Chapter 5, scanning X-ray diffraction is employed in combination with X-ray fluorescence to study the nanoscale structure and chemical composition of neuronal tissue to measure changes due to the neuropathology of Parkinson's disease.

In the future, scanning X-ray diffraction and holographic imaging will profit greatly from the increasingly brilliant radiation offered by fourth generation synchrotrons and X-ray free-electron lasers. At such facilities, the accurate delivery of a sample into the intense X-ray beam is of primary concern. In view of a potential application of scanning X-ray diffraction and holographic imaging at such modern X-ray sources, in Chapter 6, an optical stretcher has been tested as a potential sample delivery system. Surprisingly, the micromanipulation capabilities by the optical stretcher enabled also a tomographic recording of a biological cell. 


\section{X-ray diffraction and coherent imaging}

\subsection{X-ray diffraction on muscle}

It is widely considered that protein crystallography has been the driving force in the development of synchrotron radiation. However, the first beamline installed at a synchrotron was not built to determine the structure of organic compounds or proteins, but to understand in greater detail the mechanism of muscle contraction [1]. What happens when muscle actively contracts? At which point in the contraction cycle is force generated in the muscle? Such questions could not be answered with conventional X-ray sources and exigently required more intense synchrotron radiation, that enables exciting experiments which continue to deepen our understanding of the subtleties of muscle contraction.

Understanding the molecular details of muscle contraction has been on the scientific agenda since the earliest experiments with X-ray tubes in the beginning of the 20th century. Following the devastating events of the second world war and in view of the bombings of Hiroshima and Nagasaki, many physicists have turned to the politically uncharged field of biophysics [2, p. 293]. In particular the field of structural biology experienced a tremendous leap with the structure determination of the desoxyribonucleic acid (DNA) by James Watson and Francis Crick in 1953 with data from Rosalind Franklin [2, 3]. Around the same time, a strong line of research developed that targeted the intricate structure of muscle, especially because, due to the resolution limitations of visible light microscopy, there existed much debate about how force is generated in the muscle.

In 1954, Hugh Huxley with Jean Hanson and Andrew Huxley with Rolf Niedergerke jointly published two seminal articles in the journal Nature that first propelled what then became to be known as the sliding filament model of muscle contraction $[4,5]$. Even though their work primarily included results obtained using visible light microscopy and electron microscopy, it is also based on earlier recordings of small-angle X-ray diffraction studies. In the following years, X-ray diffraction became a key method in muscle research as it could be applied on a hydrated specimen without the further need for sample fixation and was therefore not prone 
to fixation artifacts. One difficulty with X-ray diffraction is, however, that it does not yield an image of the object. The contraction mechanism is explained on the basis of sophisticated models that are built such that they are in agreement with the recorded diffraction patterns.

Using a rotating anode, muscle could be studied in different states: contracted, relaxed and in rigor. To record a single X-ray diffraction pattern, recording times of several hours for equatorial reflections (from the regular packing of filaments) and days for the weaker meridional reflections (from the packing of the monomers into a helical array) were necessary. This excluded the possibility to record movies of muscle upon contraction. With the discovery of 'parasitic' $\mathrm{x}$-radiation that was generated in the bending magnets of synchrotron accelerators, its potential for muscle research did not stay unnoticed [6-8]. Since the flux of such radiation was already a factor of 10 to 100 times greater than conventional X-ray sources, it was ideally suited for muscle diffraction. The first X-ray experiment on insect flight muscle using synchrotron radiation took already place in 1971 [6], five years before the first protein crystallography experiment [9]. In the following years, more elaborate models were built and finally led to a clear picture of the mechanics of the working stroke [10-12].

Most X-ray experiments on muscle are now being carried out at synchrotrons worldwide. To the present day, several research groups are devoted to sample even finer features of the sarcomere, the contractile unit of the muscle. A single diffraction pattern can now be recorded in less then $100 \mathrm{~ms}$ and the diffraction pattern is recorded on single-photon counting two-dimensional pixel detectors at frame rates that can easily exceed 100 frames per second if necessary. The tremendous improvement in the quality and intensity of the X-ray beams enabled research groups to even sample changes in the diffraction signal under load with millisecond time resolution $[13,14]$.

To underline the hierarchical nature of muscle that ultimately makes X-ray diffraction an ideal method for its structural analysis, the structure of a single sarcomere, as reprinted in many textbooks, is shown in Fig. 1.1(A). A sarcomere is the smallest contractile unit in muscle with a length of approximately $2 \mu \mathrm{m}$ when muscle is at rest and the building block of myofibrils that traverse a muscle cell. In skeletal muscle, muscle cells fuse and form an elongated multi-cellular syncytium while the myocardium is composed of cardiac cells that contain only 1-2 nuclei and which are connected via intercalated discs. The sarcomere is composed of a parallel arrangement of actin and myosin filaments. The minus end of the polar actin fibers is attached to the Z-line (from the German word Zwischenscheibe) via 
the cross-linker $\alpha$-actinin. Myosin fibers are stabilized in the center of the sarcomere through the M-band (from the German word Mittelscheibe). The overlapping and non-overlapping region between the two filament types is termed A-band (anisotropic) and I-band (isotropic), respectively, due to their dark and light appearance in the microscope under illumation with polarized light [15, p. 1027]. Myosin, more precisely myosin II, depicted in Fig. 1.1(B) is a protein composed of two light chains, two heavy chains and two globular heads that each contain an ATP- and actin binding site and thereby can form cross-bridges with the actin filaments (also shown in Fig.1.1B). The formation of cross-bridges requires sufficiently high concentrations of sarcoplasmic $\mathrm{Ca}^{2+}$ and ATP. Binding of $\mathrm{Ca}^{2+}$ to troponin-C leads to a conformational change in the troponin/tropomyosin complex that exposes the binding sites of actin. Once ATP is bound to myosin and hydrolyzed, myosin can attach to the binding site of actin, forming a cross-bridge between the two filaments. When ADP and the phosphate are released, myosin undergoes a conformational change leading to a working stroke. Myosin can only detach from the actin filament when ATP is bound and is then allowed to return to its initial conformation. The repeated attachment and detachment with an intermittent working stroke is termed crossbridge cycling and is the basis for force generation in muscle. The crossbridge cycle is described in greater detail in [10, 16]. One should note that the sketch depicted in Fig. 1.1 does exclude important components of the sarcomere for clarity, such as titin, the largest known protein in human and that is now known to be crucial for the elastic properties of muscle cells [15, p. 1028].

Both, myosin and actin filaments, are helices with different helical repeats, see Fig. 1.1(C). An actin filament is composed of globular actin (G-actin) arranged into a helix with 13/6 symmetry, i.e. 13 monomers in 6 left-handed helical turns. In contrast, myosin is a three-stranded helix composed of 9 monomers per turn, yielding a $9 / 1$ helical symmetry.

In the A-band, the two types of filaments are arranged in an hexagonal lattice. The most prominent reflections from this lattice are the $(1,0)$ and $(1,1)$-reflection. The hexagonal lattice in the M-band and A-band is sketched in Fig. 1.1(D). The interfilament spacing $d^{(1,0)}$ typically ranges between $35.0 \mathrm{~nm}$ and $38.3 \mathrm{~nm}$ for intact rat myocardium. We have published a more extensive list with literature references online (https://irpgoe.github.io/nanodiffraction/). The intensity ratio between the two reflections is in many studies used to access the relative number of myosin motors that are attached to the actin filaments. Furthermore, it is a general rule of thumb that the lattice spacing decreases with sarcomere length [17]. 


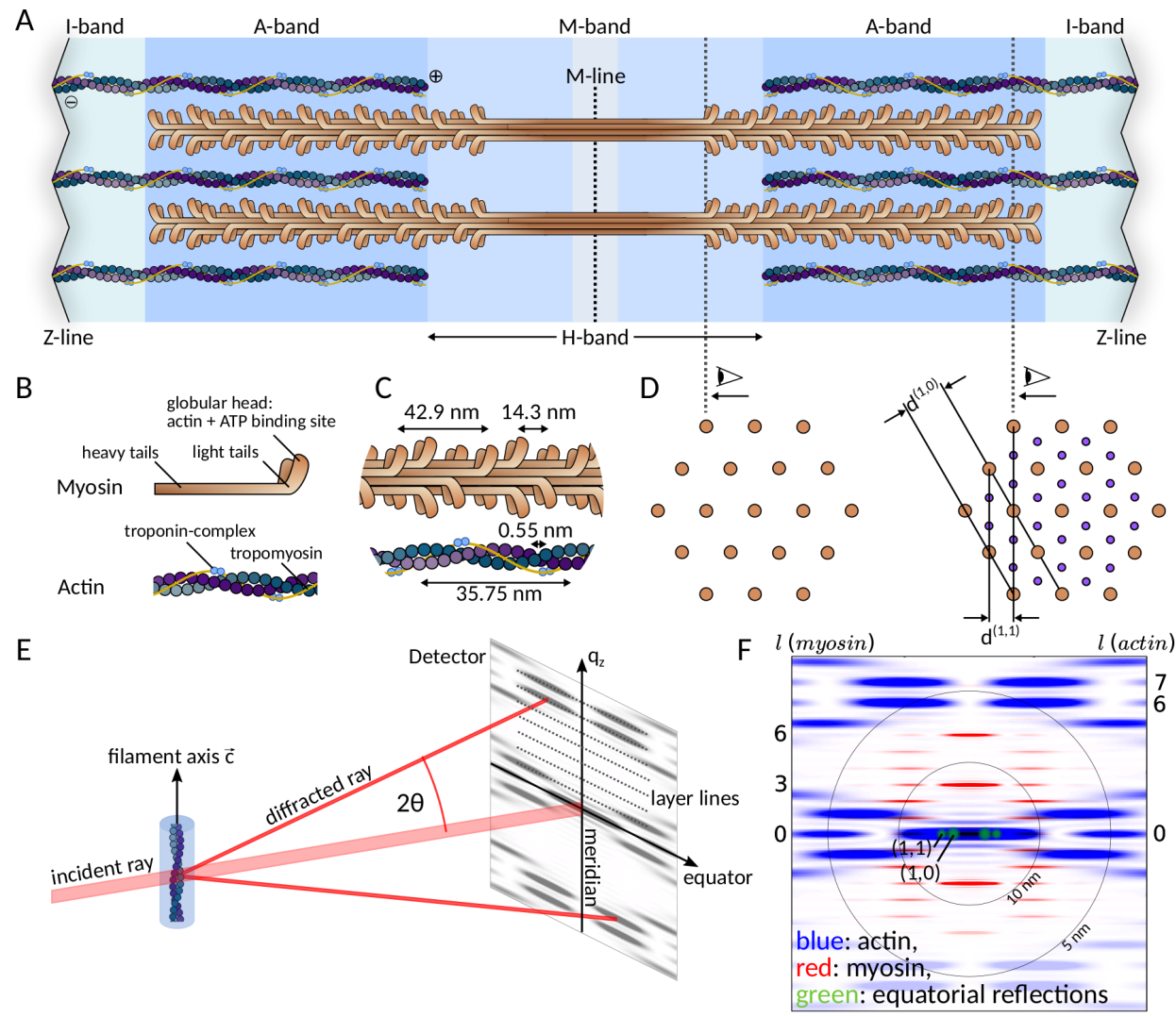

Figure 1.1.: Structure of the sarcomere and resulting layer line reflections. (A) Sketch of a sarcomere. Thick (myosin) filaments colored in orange can attach to neighbouring thin (actin) filaments. The band structure, as it appears under a microscope, is indicated in light-blue background color. (B) Close-up sketch of a single myosin molecule with a coil-coil motive forming the heavy tail and a flexible 'hinge' region, the light tails. ATPhydrolysis and actin binding takes place in the globular head region. Actin filaments are double stranded and surrounded by tropomyosin and troponin that strongly influence $\mathrm{Ca}^{2+}$ sensitivity. (C) Helical periodicities in the thin and thick filaments that give rise to meridional and off-meridional reflections. (D) View along the filament axis. (D, left) Hexagonal lattice of myosin filaments in the $M$-band. ( $D$, right) Hexagonal lattice of the myosin filaments with interdigitating actin filaments. The hexagonal lattice structure gives rise to equatorial reflections. (E) Sketch of the fiber diffraction geometry. Dashed lines indicate layer lines. (F) Simulation of the structure factor of a simplified actin and myosin filament. The merged image shows the reflections in a color code with the equatorial reflections manually added for clarity. Diffraction from the two helices were simulated using the HELIX software. 
In an X-ray diffraction experiment helical symmetry gives rise to so-called 'layer lines'. The theoretical foundation for helical diffraction is detailed in [18-21]. Here, we simply recall that layer lines are observed along the meridian at $q_{z}=(2 \pi l) / c$, where $c$ is the helical repeat or pitch and $l=0,1, \ldots$ is the layer line index. In this notation, the $z$-coordinate is along the filament axis $\vec{c}$ and $q_{z}$ is the $z$-component of the wavevector transfer $q$ defined as $q=\frac{4 \pi}{\lambda} \sin (\theta)$, where $2 \theta$ is the scattering angle and $\lambda$ the wavelength of the radiation. The general layout of a fiber diffraction experiment from which diffraction patterns on muscle fibers can be obtained is sketched in Fig. 1.1(E). Parallel to the equator, the intensity of a layer line is modulated by a sum of Bessel functions $\sum_{n} e^{i n \phi_{j}} J_{n}\left(q_{r} r_{j}\right)$ where $r_{j}$ denotes the radial position and $\phi_{j}$ the orientation of the $j$-th monomer in cylindrical coordinates. The order of the Bessel function $n$ is subject to the selection rule $l=n s+m p$, where $n$ and $m$ are integers, $s$ is the number of helical turns per repeat and $p$ is the number of monomers per repeat. The helical symmetries mentioned above are approximations to the actual structure. Most notably, in the case of myosin the helical selection rule should forbid all meridional reflections except for repeats that are multiples of a fraction of the monomer spacing, i.e. where $1=3,6,9$, etc [22]. In other words, because the crystallographic repeat is exactly three times the monomeric repeat, only the monomeric repeat should be observed. The fact that in e.g. relaxed human muscle these 'forbidden reflections' can be observed shows the deviation from ideal helical symmetry. This deviation is removed, i.e. symmetry is restored, when the muscle is brought into rigor [23].

The myosin and actin reflections as well as the $(1,0)$ and $(1,1)$ equatorial reflections are shown in Fig. 1.1(F). The myosin and actin reflections were simulated from a simplified theoretical model of the structure using the HELIX software [24]. The most intense layer line reflections are labeled with the layer line index $l$. In the literature, as a shorthand notation, actin layer lines are labeled as A1, A2, etc. while myosin layer lines are indexed as M1, M2, etc., where A1 corresponds to first actin layer line at $1 / 35.75 \mathrm{~nm}^{-1}$ and M1 to the first myosin layer line at $1 / 42.9 \mathrm{~nm}^{-1}$. Equatorial reflections (green) were simply sketched onto the simulated pattern to illustrate their respective location in the diffraction pattern.

It is not surprising that earliest experiments were conducted on skeletal muscle since single fibers can be easily extracted and prepared. X-ray diffraction was only recently applied to cardiac muscle due to the very delicate task of extracting isolated muscle fibers from the heart, now yielding valuable insight into the the molecular basis of the Frank-Starling law [25, 26]. For this purpose, a trabaeculae of the heart can for example be isolated and used. In such an experiment information 
on the cardiac muscle is obtained from a single location in the heart and due to large beam diameters of around $100 \mu \mathrm{m}$ the recorded signal corresponds to an average over many muscle cells in the muscle fiber.

\subsection{Adding real space resolution}

Although a large beam ensures a high signal level, more efforts are still being invested into the generation of smaller X-ray beams, in part also to collect similar diffraction information but now from a very localized spot in the sample [27]. With recent advances in focusing optics for X-rays, beams with nanometer spot sizes can be produced to investigate the local structure of materials. By raster scanning these so-called 'nanoprobes', diffraction information can be combined with real space information to e.g. observe variations in the diffraction signals.

Related to previous research on muscle, this now adds real space resolution and can possibly be used to identify local variations in the muscle structure. Even beams with a moderate focal spot size in the range of $10 \mu \mathrm{m}$ can still be of tremendous use in structural imaging. By lateral scanning of the sample through the focus of an X-ray beam one or multiple features of the diffraction pattern can be analyzed and an image of structural parameters can be formed as sketched in Fig. 1.2. In addition to such parameter maps sketched in Fig. 1.2(B) it is also common to investigate isolated diffraction patterns or an ensemble average over all patterns. Such nano- or microbeam scanning experiments have been applied on a vast range of samples. In the case of biological matter, most studies have focused on textured samples such as wood [28-31] or bone [32-37]. The scanning diffraction principle can even be extended to three-dimensional structures as demonstrated in [38-40] but prohibitively long recording times thus far exclude the use for soft biological specimen.

With a reduction of the beam size down to the nanometer range, it was demonstrated that scanning X-ray diffraction is even suited to analyze the structure of isolated biological cells [41-44]. It is the primary aim of this thesis to use the methods and techniques that have been developed in the previous body of work to investigate the sarcomeric organization in single heart muscle cells (cardiomyocytes). To exclude artifacts from sample processing and radiation-induced damage, we utilized different preparation methods and optimized experimental parameters to make most efficient use of photons. In addition to the use of neonatal rat cardiomyocytes, we have included human induced pluripotent stem cells (hiPS) derived into cardiomyocytes and adult cardiomyocytes from mice in this study to draw 
A

$A$

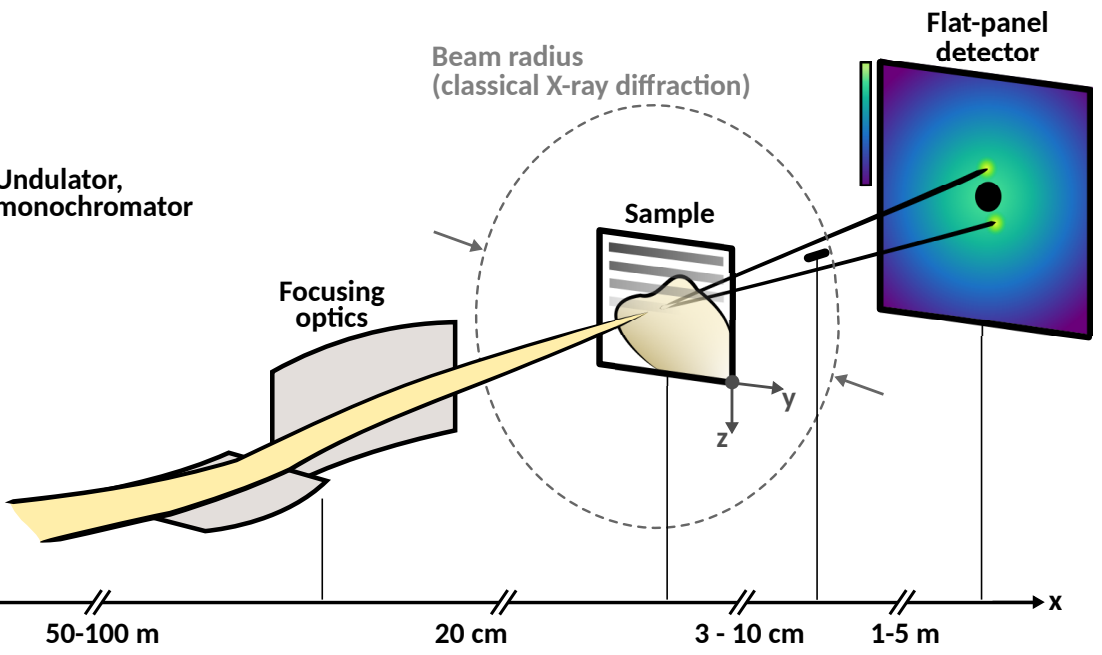

\section{B Recorded scattering}
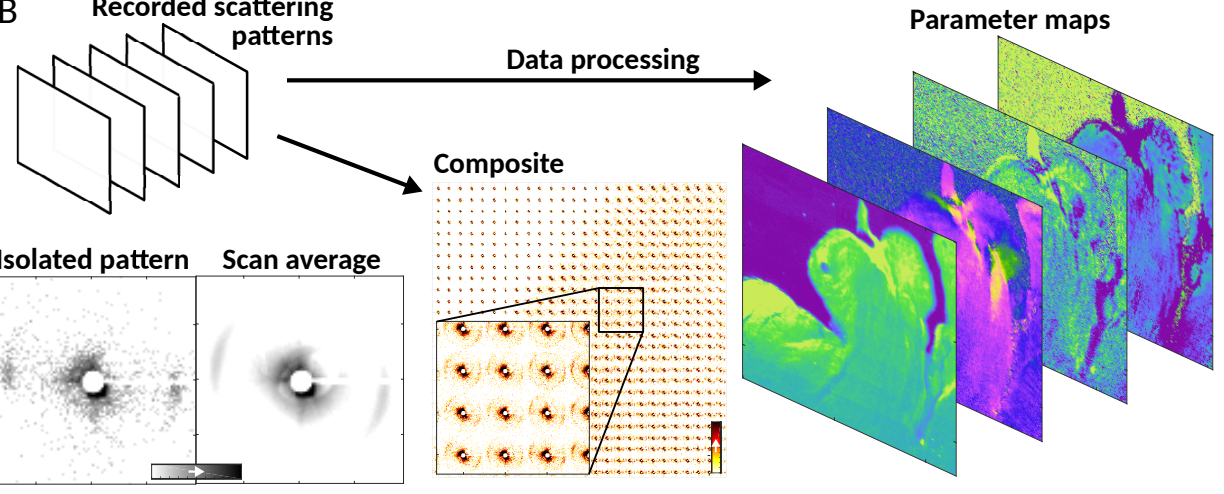

Figure 1.2.: Principle of scanning $X$-ray diffraction. (A) $X$-rays are focused using either elliptical focusing mirrors as sketched in the figure or lens-based focusing such as compound refractive lenses. In contrast to classical diffraction, the focal spot is orders of magnitude smaller and the sample is scanned through the X-ray focus and the scattered $X$-rays are recorded on a flat-panel pixel detector. The primary beam is blocked by a beam stop. (B) The recorded diffraction patterns can for example be summed or displayed with respect to their location in the scan. If a certain feature of the diffraction pattern is extracted, it can be represented as a map, hence, making scanning $X$-ray diffraction essentially an imaging technique. 
comparisons between different developmental states of the cells.

In fact, data recorded on isolated cells did not resemble the typical X-ray diffraction patterns observed on muscle fibers by means of classical X-ray diffraction. In the case of an absence of typical reflections, the small-angle X-ray scattering (SAXS) signal still encodes correlations in the electron density and can be exploited to extract statistical and structural information such as the myofibril orientation in the cell. We have observed equatorial reflections only for hydrated adult cardiomyocytes and in tissue sections from adult heart. The absence of a diffraction signal of sarcomeric origin is intriguing because one can hypothesize that the developmental state of the cardiomyocyte does indeed influence the presence of a small-angle diffraction signal. In Chapter 4, we present evidence that support the above hypothesis and discuss the results in view of possible artifacts stemming from the, in many cases, intensive sample preparation and other factors that could possibly influence the scattering signal.

In contrast, in thin sections from cardiac tissue equatorial reflections can be detected at every scan point and with larger areas that can be covered, the treatment of increasing amounts of diffraction data has become increasingly demanding. In early experiments on cardiac tissue, only a small section of the several $\mathrm{mm}^{2}$ spanning sample could be imaged. This limitation was recently overcome by the implementation of a continuous scanning mode [45] which greatly improved the speed at which the sample could be imaged. In combination with novel, fast X-ray detectors, scans with more than one million scan points are now possible. One should keep in mind that every scan point ${ }^{1}$ features a full $2 \mathrm{D}$ diffraction pattern and data storage can easily exceed one terabyte per scan. To process such large amounts of data it was of primary concern to develop and implement robust analysis routines and establish a workflow that makes efficient use of data. The developed tools have been bundled into a nanodiffraction toolbox that has recently been made publicly available [46].

The developed tools, the continuous scanning mode and the optimization of scan parameters based on experience from earlier synchrotron experiments has led to the routine application of the technique to biological cells and histological sections. The study presented in Chapter 3 is the earliest example for such an experiment on a hydrated histological cardiac section and can now be easily extended to characterize cells and tissue from a diseased heart. It has been shown that many cardiomyopathies can lead to a drastic change in the mechanical properties of the

\footnotetext{
${ }^{1}$ In continuous scanning a scan point is defined as the distance that is covered by the $\mathrm{X}$-ray beam during one exposure.
} 
heart and should therefore be linked to the molecular architecture of the actomyosin complex. Variations in the structural integrity of the sarcomere due to cardiomyopathies should therefore be observed in a diffraction contrast map.

Aside from imaging and localizing structural variations, the analysis of many isolated cells is a relevant extension of this work as it enables a complete statistical evaluation of a larger population of cells and correlations between ensembles of cells can be evaluated on a statistical basis. In Chapter 4 we have used the myosin filament spacing as a structural parameter and show how it is affected by the use of different chemical fixatives and mouse treatment before the cells were isolated. A histogram representation can in this respect condense the large amount of data in a very concise fashion and still convey most of the information. This has been shown to be equally true for the data analysis on tissue as demonstrated in Chapter 3.

\subsection{Mapping of neuronal tissue}

Thus far, we have concentrated on the analysis of small-angle diffraction from cardiac cells and tissue. However, the earliest scanning diffraction experiments on soft biological samples were performed on neuronal tissue [47]. This is owed to the presence of very intense reflections due to the lamellar stacking of lipids in the myelin sheath surrounding the axons of neurons in the brain and whose integrity is vital for neuronal function. The structural analysis of the myelin sheath is motivated by the fact that demyelination of axons is the leading cause for multiple sklerosis (MS), although the molecular details of myelination and demyelination are still being actively researched [48].

As for cardiac structure, the general understanding of myelin structure is based on results stemming from a combination of electron microscopy and X-ray diffraction experiments $[49,50]$. X-ray diffraction has proven particularly useful to assess the phase behavior of the myelin sheath [51]. By means of scanning diffraction with micro-focused X-ray beams, it was additionally found that the structure of myelin does vary to a significant degree throughout the tissue, both in fixed and unfixed tissue [52]. Together with the group of Prof. Dr. Paul Lingor from the University Medical Center Göttingen, we have utilized scanning X-ray diffraction to image the structure of myelin in neuronal tissue in combination with scanning X-ray fluorescence to map out the metal content of dopaminergic neurons in the substantia nigra $(\mathrm{SN})$ pars compacta. It was the aim of the study described in Chapter 5 to, in one part, determine the axon orientation and myelin lamellar spacing by analysis of the small-angle scattering signal. In a second part, X-ray 
fluorescence microscopy was used to compare the concentrations of intracellular metal ions between a Parkinson's diseased (PD) patient and a control patient and to possibly localize protein inclusions.

X-ray fluorescence has already been successfully used in Parkinson research, due to its high sensitivity to trace elements and the added real space resolution down to the nanometer range [53-55]. The pathology of PD, the second most common degenerative disease, is divided into two main processes: (a) the degeneration of dopaminergic neurons, and (b) the misfolding and aggregation of $\alpha$-synuclein. Trace elements play a particularly important role in the pathology of Parkinson's disease. It was for example shown that iron content is increased in PD patients [56]. Increased amounts of trace metals can lead to oxidative stress through the production of free radicals that can subsequently oxidize other cellular components such as lipids or cholesterol [57]. Also non-redox active metals can become cytotoxic at high concentrations and determining the concentration of such ions, most notably copper and iron but also calcium and zink, is important in clarifying the role of such metal ions in the development of neurological diseases such as PD, but also Alzheimer's disease and Huntington's disease [58].

Oxidative stress triggered by an increase of reactive oxygen species formed by redox active metals might also lead to protein aggregation. The misfolding and aggregation of the protein $\alpha$-synuclein and variants thereof, as it occurs along the neuropathology of PD, are termed Lewy bodys. Iron, besides its indirect effect on aggregation through the production of reactive oxygen species, is also shown to be the leading cause of aggregation through direct binding to native $\alpha$-synuclein. Iron has therefore been the key target in metal imaging and quantification as it is closely linked to the pathology of the disease. X-ray fluorescence microscopy is well suited to image and quantify transition metals since it offers high spatial resolution with a very performant detection limit of about $0.1 \mu \mathrm{g} \mathrm{g}^{-1}$ to $1 \mu \mathrm{g} \mathrm{g}^{-1}$ [58]. In many cases, it is combined with other techniques to map the content of metal ions such as Particle Induced X-ray Emission (PIXE), Electron Energy Loss Spectroscopy (EELS) and micro-X-ray Absorption Spectroscopy ( $\mu$-XAS).

\subsection{Coherent imaging with hard X-rays: From cells to tissues}

Thus far we have not taken into account the ever increasing coherence of X-ray beams produced at synchrotron radiation facilities. In the past decades, X-ray 
imaging techniques have tremendously benefited from more coherent nanofocused X-ray beams. Most notably, lensless imaging methods could be developed were an image is formed when the sample is placed into the focus or defocus position of a coherent diverging beam. Placing the sample into the focus position results in the far-field diffraction pattern being recorded on the detector. The Fraunhofer pattern can be inverted by suitable reconstruction algorithms to yield an image of the object. This approach, termed coherent diffractive imaging (CDI) [59], and refined variants thereof such as ptychography [60], can effectively be used to image microscopic biological samples such as cells [61, 62]. In contrast, in full-field holographic imaging the sample is placed into the defocus position of the diverging beam and by interference of the scattering waves with the probing beam a hologram of the sample can be recorded. This approach, also termed near-field inline holography or holographic imaging is most sensitive to gradients of the electron density and phase shifts of the sample.

The different experimental configurations in scanning diffraction, ptychography, coherent diffractive imaging and holographic imaging are sketched in Fig. 1.3. Methods that involve scanning such as scanning X-ray diffraction and ptychography can be combined with X-ray fluorescence microscopy through the straightforward addition of a suitable energy-resolving X-ray detector. In plane-wave CDI a pinhole is used with an opening that is smaller than the transverse coherence length $\xi_{r}$ of the illumination to select only the coherent portion of the beam. Conversely, without any pinhole, one could also use a sample that is smaller than $\xi_{r}$. For near-field holography, a divergent X-ray beam with sufficient lateral and transversal coherence is required that can be generated in several ways. In this work, we have used the Göttingen endstation GINIX available at beamline P10 of the German electron synchrotron DESY, where the X-ray beam is coupled into an $\mathrm{X}$-ray waveguide for coherence filtering [63]. The quasi-point source illumination is then used for propagation-based phase-contrast imaging, i.e. holographic imaging, or tomography. In holographic imaging, the field of view can be adjusted to the size of the object by choosing an appropriate defocus distance and the dose is spread over the entire field of view, making the technique appropriate for imaging of living cells [64]. The holographic projections are inverted by phase retrieval algorithms. In the most simple case, the phase shift of the object, that also encodes the electron density, can be retrieved in a single inversion step using for example the contrast transfer function [65] or transport of intensity equation [66, 67]. In many cases, due to rather stringent assumptions about the sample and the probing beam, this procedure can introduce artifacts that can be circumvented 

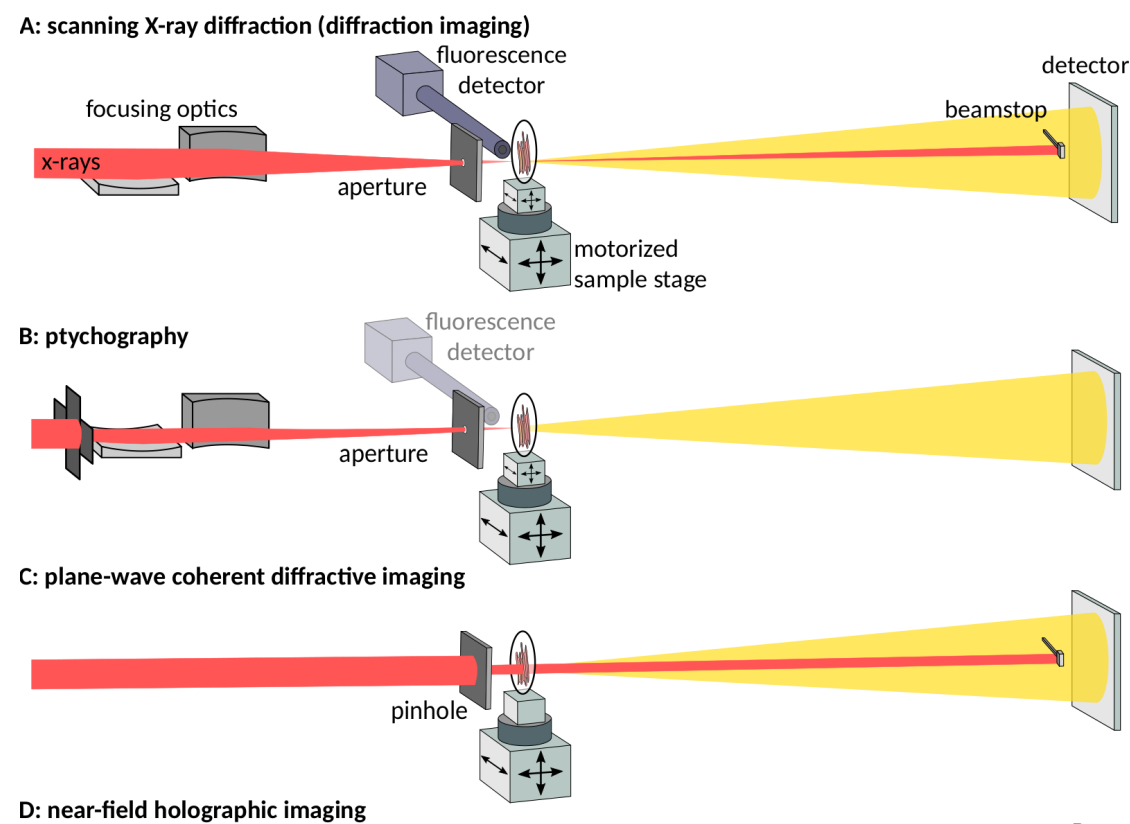

D: near-field holographic imaging

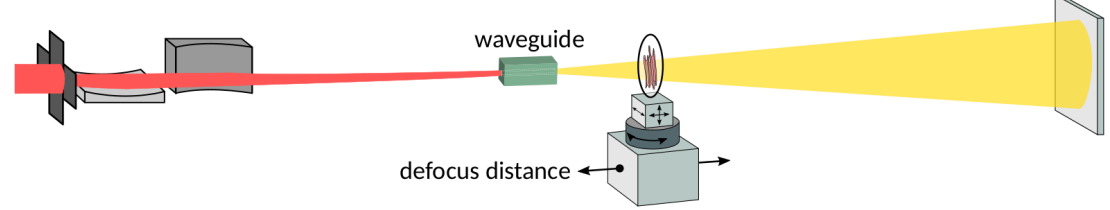

Figure 1.3.: Sketch of different $X$-ray imaging modalities. (A) Scanning X-ray diffraction. A fluorescence detector can be used for additional XRF measurements. (B) For ptychography the coherent portion of the beam is used. The sample is then again scanned through the focus of the beam, which makes ptychography also compatible with XRF recordings. (C) In plane-wave coherent diffractive imaging a pinhole was originally used for coherence filtering. The pinhole can be omitted if the sample is smaller than the coherence length of the illumination. (D) Near-field holographic imaging. The sample is placed into the defocus of a coherent divergent illumination that can be generated by e.g. coupling a pre-focused beam into a waveguide for coherence filtering.

by iterative algorithms. Such algorithms are designed to adapt an initial guess for the object to the measured intensities and a solution is searched that is compatible with the measurement constraint set [68]. The solution can then be refined or the search speed and robustness be improved by introducing other constraint sets which however require further knowledge of the sample or the optical transfer function. Both, single step and iterative reconstructions can be improved upon by including measurements at multiple defocus distances [69]. 
With holographic imaging, a resolution of typically $50 \mathrm{~nm}$ can be achieved. Beyond this limit, experimental factors such as vibrations are currently limiting the accessible real space resolution. Scanning X-ray diffraction on the other hand is sensitive to structural periodicites below approximately $50 \mathrm{~nm}$, limited at low $q$ by the size of the beamstop and at high $q$ ultimately by the signal level or the largest scattering angle that can be recorded with the detector. A combination of both techniques can therefore greatly extend the resolution range covered by a single method alone. In view of biological cells, a combined approach would perfectly match the lengths scale of a single cell, ranging from several micrometers down to the nanometer scale.

In Chapter 2 we show that holographic imaging can be rather easily combined with diffraction imaging, with little realignment of the experimental setup. From these experiments we concluded that a combined approach is even of greater benefit than simply the additional length scales covered: (i) maps of structure parameters can be correlated with an image of the retrieved electron density, (ii) full-field imaging can guide the scanning diffraction experiment and eliminate the need for intensive 'search scans', (iii) changes in the structure due to radiation damage can be observed and monitored by later inspection of the holographic reconstruction, and (iv) holographic imaging can be most easily extended to tomography.

Especially in view of the advent of even brighter fourth generation synchrotron sources that will operate at or near the diffraction limit and the increasing availability of X-ray free electron lasers (XFEL), radiation damage will be a great limitation and X-ray diffraction experiments, also at synchrotron sources, will have to rely on collecting a diffraction signal before the structure starts to decay. This diffract before destroy method is already used at XFELs to outrun radiation damage and will most certainly be of use at more intense synchrotron sources. This however makes guidance and careful planning of a scanning diffraction experiment increasingly important and holographic imaging can in this respect serve as a tool to inform the scanning diffraction experiment. In this respect it might also be beneficial to collect diffraction patterns only at isolated locations on the sample that can be selected based on electron density maps.

Coherent imaging techniques such as holographic imaging, now well established in synchrotron research, will also play a key role at future XFEL sources. The highly coherent and intense beam can deliver $10^{10}$ up to $10^{13}$ photons per X-ray pulse. At such intensities, a single hologram can be recorded in only a single shot. The short exposure times in holographic imaging experiments further eliminate any motional blurring and therefore increase the signal-to-noise ratio drastically. 
Limitations in resolution imposed by vibrations are hereby also removed. One key technical difficulty is however the accurate positioning of objects in the X-ray beam. For highly efficient usage of precious sample material it would be of great benefit to deliver a single sample into the focal spot, then image the sample and refill the focal spot with an undamaged, new sample. At XFELs, jet- or droplet injection systems [70] are used for this purpose.

To pave the way for a potential application of holographic imaging to biological cells at an XFEL source, we have tested the use of an optical stretcher as sample delivery system for synchrotron and XFEL research. The system and initial tests described in Chapter 6 have shown that it offers great potential not only in combination with holographic imaging but also for diffraction studies. An optical stretcher is well suited to manipulate micrometer-sized objects such as cells and can not only be used for trapping but also to elastically deform the trapped particle or cell. This opens up interesting possibilities, once again related to the structural diffraction studies on cardiomyocytes, by probing the local structure now under deformation and stress. The micromanipulation capabilities of an optical stretcher have also offered the possibility to perform tomographic experiments on single biological cells in suspension. Even though the three-dimensional reconstruction of a macrophage loaded with barium grains described in Chapter 6 is still poorly resolved and far from artifact-free, it still illustrates the usefulness also in view of three-dimensional imaging of micrometer-sized particles with hard X-rays.

\subsection{Future directions}

With respect to cardiomyocytes, already without an optical stretcher, we could perform holographic imaging experiments on single adult cardiomyocytes under hydrated conditions with sufficient contrast to reconstruct the electron density of the cell. In the future, we expect that holographic imaging studies will be extended to (i) image living, actively contracting cardiomyocytes, and (ii) retrieve the full $3 \mathrm{D}$ electron density of a cardiomyocyte by means of phase-contrast holographic tomography. Tomographic imaging of single cardiomyocytes will integrate well with previous results obtained on biopsies from heart tissue by means of holographic tomography, see for example [71]. Due to limitations on the sample size, in particular in synchrotron holographic tomography, a full heart can be scanned sectionwise and will be stitched together to produce a full tomogram of a heart. This would demonstrate that, by means of hard X-ray holographic tomography, the heart can be imaged in $3 \mathrm{D}$ on all length scales, from the cyto-scale to the organ scale. In 
$[71,72]$ it was already shown that the myofiber orientation can be retrieved from such datasets. We expect that these algorithms will be further improved upon in order to be able to reconstruct the myofiber orientation in 3D. For biological or medical research it would be largely beneficial to obtain a tomogram from the entire heart (potentially including the myofiber orientation), since in the context of cardiac diseases, such studies may provide important 3D structural insight into the cyto-architecture underlying the pathologies.

Multi-scale imaging does not necessariliy need to be performed with a single imaging method. Indeed, the added benefit from combining two different modalities can be greater than the sum of the two [73]. We have primarily used visible light fluorescence microscopy for this purpose as it is a very well established imaging method that offers a distinct, highly specific contrast that does not exploit the electron density. By such means we could verify the intactness of the sample after an intensive sample preparation and observe that the small-angle scattering signal from cell preparations is primarily stemming from the nucleus and the cytoskeletal network of the cell. Now, with the availability of super-resolution microscopy, the added benefit will be even greater and future, less method-driven research questions, will rely on the joint application of super-resolution microscopy and X-ray diffraction imaging with holographic imaging.

This thesis did therefore not only further establish a single method - scanning X-ray diffraction - for cellular imaging but also integrated it with other imaging modalities. Regarding the scanning diffraction technique, there are still many improvements that can be made to increase the sensitivity and minimize the impact of radiation damage. The latter is of great concern especially when beam sizes are reduced down to the nanometer range. In Chapter A in the Appendix we discuss the issue of radiation damage in greater detail and outline our latest efforts in the development of a cryogenic chamber that is specifically designed for scanning diffraction experiments. With latest experimental improvements now under way, important steps have already been taken in establishing scanning X-ray diffraction for soft matter samples. The articles reproduced in Chapter 4 and 5 further investigated the capabilities and limitations of the combination of imaging methods. These surveys formed a solid footing for further studies. They also provide a link to a medical application of the technique. It is to assume that this link will be studied more closely in the future.

At last, it is fascinating to observe how the optimization of the scanning X-ray diffraction technique for soft biological specimen was strongly driven by an interest in the molecular structure of muscle, in a similar fashion as classical X-ray 
diffraction developed by the scientific intereest in studying the architecture and function of muscle fibers in ever increasing detail. 


\title{
2. Combined scanning X-ray diffraction and holographic imaging of cardiomyocytes
}

\author{
J.-D. Nicolas, M. Bernhardt, M. Krenkel, C. Richter, S. Luther and T. Salditt
}

Reproduced from Journal of Applied Crystallography 50, 612 - 620 (2017).

This article presents scanning small-angle X-ray scattering (SAXS) experiments on the actomyosin assemblies in freeze-dried neo-natal rat cardiac muscle cells. By scanning the cells through a sub-micrometer focused beam, the local structure and filament orientation can be probed and quantified. To this end, SAXS data were recorded and analyzed directly in reciprocal space to generate maps of different structural parameters (scanning SAXS). The scanning SAXS experiments were complemented by full-field holographic imaging of the projected electron density, following a slight rearrangement of the instrumental setup. It is shown that X-ray holography is ideally suited to complete missing scattering data at low momentum transfer in the structure factor, extending the covered range of spatial frequencies by two orders of magnitude. Regions of interest for scanning can be easily selected on the basis of the electron density maps. Finally, the combination of scanning SAXS and holography allows for a direct verification of possible radiation-induced structural changes in the cell.

\subsection{Introduction}

Our current understanding of force generation in muscle is to a large extent based on the structural information on the highly ordered actomyosin assembly available at molecular resolution. Decades after the structure of the sarcomere with its functional actomyosin complexes was first elucidated by classical X-ray diffraction studies of muscle fibers [22, 74], synchrotron radiation is still used actively to study muscle contraction mechanisms in skeletal and cardiac muscle of vertebrates and 
invertebrates [14, 75-78].

In these experiments, structural information is averaged over macroscopically large volumes of the tissue, with diffraction volumes containing up to thousands of muscle cells. Contrarily, recent progress in X-ray optics has enabled diffraction experiments with spot sizes in the sub-micrometer range, well suited to illuminate only selected organelles of a single cell. Fourier components corresponding to nanometer-sized structures can be measured, like in a standard small-angle diffraction experiment, but scaled down to a sample volume corresponding to a specific part of a cell. For actin and other protein networks, one may deduce information on the orientation, density and bundling of filaments by cross-linkers [79], and on the formation of complexes and structures associated with motor proteins. Such smallangle diffraction experiments employing tightly focused X-ray beams were recently applied to study the actin network of the amoeba Dictyostelium discoideum [43], a well known model system for cellular migration.

In general, small-angle X-ray scattering (SAXS) maps of cells recorded by laterally scanning the cell in the focus position result in a two-dimensional raster image with a pixel size of the order of the beam size, typically $200 \mathrm{~nm}-300 \mathrm{~nm}$ in recent experiments $[42,43]$. A SAXS map can represent a variety of observables, such as phase shift (i.e. projected density), ion concentration from fluorescence yield, small-angle scattering intensity extracted from the far-field pattern (dark field) and other structural parameters derived from the full two-dimensional diffraction pattern.

Using micro- and nano-focused beams for X-ray diffraction, our long-term goal is to elucidate the fine structure of the protein networks forming the sarcomere in muscle cells without large averages over the tissue. Thus, it becomes possible to trace anisotropies and distribution functions and to distinguish the inter- and intra-cellular aspects of protein complex formation. This can be understood as an extension of the classical structure analysis of muscle to the level of single cells, which is important to understand the emergence of the highly ordered actomyosin structures.

As a first step towards these goals, we show in this work that micro-beam diffraction, i.e. scanning SAXS recordings, can be advantageously combined with lensless coherent imaging of the cell's (projected) electron density. In this respect, a fullfield low-dose holographic image is recorded by moving the sample downstream from the focal plane, as sketched in Fig. 2.1. By selection of the defocus position $z$, the illuminated area can be tailored to the size of the cell or to a region with several cells. Holographic recordings are analyzed by a suitable phase retrieval al- 
gorithm, here based on the modified iterative hybrid-input-output algorithm [80] and for the first time extended to multiple defocus distances [65].

Experimentally, as we demonstrate here, one can switch by a simple change of experimental parameters (defocus position, detectors, insertion of waveguide module and beamstop) between the focal-plane setting optimized for high resolution diffraction and the defocus setting optimized for full-field coherent imaging.

We have previously implemented a similar combined approach based on scanning diffraction and imaging [62], which utilized ptychographic phase retrieval [60, 61, 81]. However, the present holographic approach allows for electron density maps with significantly less dose, which is even compatible with live cell imaging [64] and is more easily extended to tomography, since no scanning is required for the holographic phasing. Compared to a standard scanning X-ray diffraction experiment (i.e. raster scanning the sample through the focused X-ray beam), the combined approach offers the following advantages: (i) correlative microscopy images of projected density and diffraction observables are obtained, (ii) a region of interest can be quickly identified by the full-field overview scan, and (iii) possible structural degradation by beam damage can be checked by investigation of changes in the projected electron density.

Furthermore, we point out the advantage of scanning diffraction with micro- or nanometer focal spot sizes, compared to performing only a lensless imaging experiment, in assessing structural information at length scales well below the pixel sizes of an imaging experiment down to the nanometer range. This is brought about by the intrinsic averaging process within the illuminated volume as well as the relaxed sampling and signal-to-noise criteria when performing the analysis directly in reciprocal space without any inversion. Radiation-induced effects can be mitigated by means of cryogenic protection and fixation of biological samples [82], as has been successfully demonstrated in a wide range of experiments by means of, for example, scanning transmission X-ray microscopy [83-86], coherent X-ray diffraction imaging [87-91], X-ray ptychography [92-94] and X-ray fluorescence imaging $[53,95]$.

The manuscript is organized as follows: Sample preparation and handling of freezedried neo-natal rat cardiac muscle cells (cardiomyocytes) is described in Sec. 2.2, which includes a brief description of the experimental imaging modalities at the micro- and nano-focus setup at the P10 beamline. In Sec. 2.3, micro-beam diffraction experiments are analyzed with respect to scattering intensity and orientation, complemented by analysis of the local structure factor recorded from different cellular compartments (regions of interest). Holographic recordings on single car- 
(a)

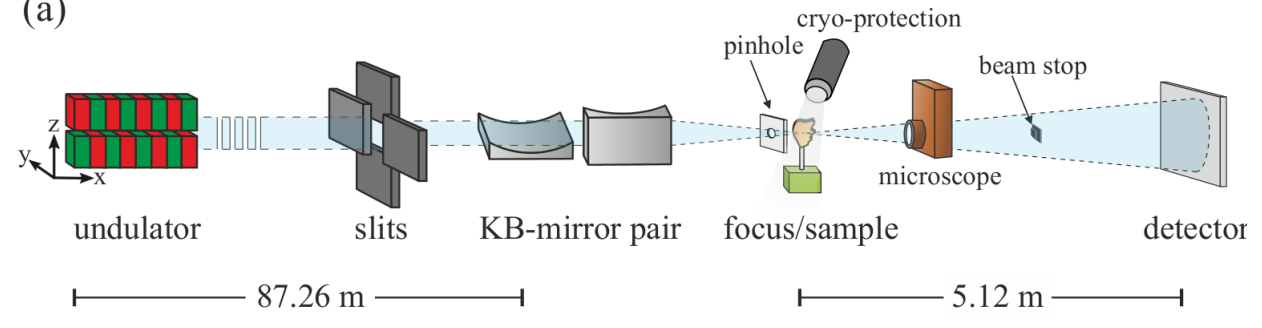

(b)
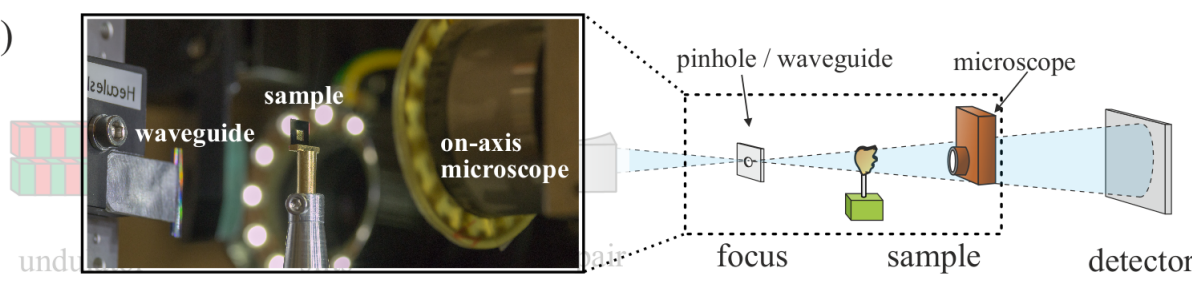

Figure 2.1.: Diffraction and imaging modalities at the micro- and nano-focus instrument of the coherence beamline P10 (PETRA III storage ring)[63]. (a) Scanning SAXS setup: the partially coherent $X$-ray beam, predefined by $K B$ entrance slits, is focused onto the sample using a pair of KB mirrors. Two consecutive soft-edge apertures remove perturbing $K B$ tails and the SAXS scattering intensity is recorded by a pixelated detector protected by two successive beamstops. Freeze-dried cells are cryo-protected during SAXS scanning. (b) Holographic imaging setup: the sample is moved to a defocus position while the KB beam is coupled into a two-dimensional waveguide for coherence filtering. After initial waveguide alignment with the pixelated detector to maximize photon flux, high-resolution holograms are acquired by an sCMOS camera. In both cases, the sample may be viewed in a fully translatable on-axis video microscope for initial alignment and supervision. $A$ drilled hole in the objective enables in situ observation during $X$-ray exposure.

diomyocytes and the reconstruction algorithms are described in Sec. 2.4, and the complementary nature of the two approaches, inverse imaging and micro-beam diffraction, is discussed. The article closes with a summary and a brief discussion of the outlook for follow-up experiments.

\subsection{Materials and methods}

\subsubsection{Sample preparation}

Neo-natal rat cardiomyocytes from wild-type rats were isolated, suspended in nutrition medium [Dulbecco's Modified Eagle Medium DMEM-F12, HEPES, no phenol red (Thermo Fisher Scientific Inc.); added 10\% fetal bovine serum and $1 \%$ 
penicillin/streptomycin] and then diluted for sample preparation (a typical con-

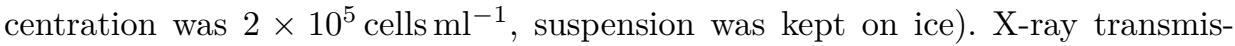
sive $\mathrm{Si}_{3} \mathrm{~N}_{4}$ windows $(1.5 \mathrm{~mm} \times 1.5 \mathrm{~mm}$ membrane size, $1 \mu \mathrm{m}$ thickness, Silson Ltd) serving as substrates were plasma-cleaned (Expanded Plasma Cleaner PDC-002, Harrick Inc.) for 5 min, transferred into Petri dishes and pre-coated by a drop of $25 \mathrm{\mu l}$ coating solution $\left(0.5 \%\right.$ fibronectin and $0.08 \%$ gelatine in sterile $\left.\mathrm{H}_{2} \mathrm{O}\right)$. After a short incubation period of approximately two hours at $310 \mathrm{~K}$ and $5 \% \mathrm{CO}_{2}$, substrates were either submersed in or covered by a drop of cell suspension. Samples were subsequently incubated, enabling cell settlement and to promote cell adherence onto the substrate. Wells were then flooded with nutrition medium for overnight cultivation. In order to find the ideal time for fixation, samples were controlled steadily by visible light microscopy. On the day of sample fixation, all samples were examined in detail under a microscope (Zeiss Observer.Z1, Carl Zeiss $\mathrm{AG}$ ), identifying beating single cells on every substrate.

In order to correlate X-ray data to actomyosin fiber networks, samples were fixed (9\% formaldehyde in phosphate-buffered saline), permeabilized ( $0.5 \%$ Triton X100, Sigma-Aldrich Co. LLC), and labeled (Phalloidin Alexa 488 and Hoechst 33342 , Invitrogen, respectively) for F-actin and DNA (to identify the nuclei). Visible light fluorescence images were recorded in detail (Zeiss Observer.Z1) before and after the freeze-drying process. In this step, cells were identified as cardiomyocytes via the weakly striated structures in the fluorescence images.

After fixation, samples were vitrified by rapid plunging of the specimen from a water-saturated atmosphere $(293 \mathrm{~K}$ relative humidity $\geq 90 \%)$ into a cold liquid ethane/propane bath (typically about $78 \mathrm{~K}$ ) using a Leica grid plunger (Leica EM GP, Leica Microsystems). For freeze-drying, samples were transferred into a homebuilt vacuum chamber while being kept under cryogenic conditions. The samples remained in the evacuated chamber for several days, ensuring the sublimation of amorphous ice. To prevent rehydration under ambient conditions, all samples were stored in a desiccator until right before the sample was mounted onto the sample stage.

\subsubsection{Experimental parameters and instrumentation}

Scanning SAXS and holographic imaging experiments were performed at the coherence beamline P10 of the storage ring PETRA III (Desy Photon Science) using the Göttingen Instrument for Nano-Imaging with X-rays (GINIX) [63, 96]. Using a $\mathrm{Si}(111)$ double-crystal monochromator, an energy of $8.0 \mathrm{keV}$ was selected. For the 
scanning SAXS experiment ( $c f$. Fig. 2.1a), the Kirkpatrick-Baez (KB) entrance slits were tuned to $0.4 \mathrm{~mm} \times 0.4 \mathrm{~mm}$. Side lobes of the $\mathrm{KB}$ beam were removed with two successive soft-edge apertures $(0.4 \mathrm{~mm} \times 0.4 \mathrm{~mm})$, yielding a very compact focal spot of about $350 \mathrm{~nm}$ (horizontal) $\times 430 \mathrm{~nm}$ (vertical) (FWHM). SAXS scattering data were recorded in this setup on a two-dimensional pixel detector [172 $\mu \mathrm{m}$ pixel size, 1 pixel point-spread function (FWHM), Pilatus 300K, Dectris, Switzerland] $5.12 \mathrm{~m}$ downstream of the sample, protected by two beamstops loosely and tightly fitting the primary beam. The sample was mounted onto a sample tower consisting of stepper motors (PI miCos $\mathrm{GmbH}$ ) and a high-precision piezoelectric stage (SmarAct) for accurate lateral scanning through the focus of the KB beam. Custom-built hardware-triggered data acquisition and motorization allowed raster scanning of the sample in a continuous mode, significantly reducing the recording time needed. Each scan point (with a typical raster scan speed of $5 \mu \mathrm{ms}^{-1}$ ) was illuminated for $0.1 \mathrm{~s}$ with continuous illumination. During SAXS acquisition, the sample was cryogenically cooled in a nitrogen cryostream (Oxford Cryosystems) to minimize radiation damage by limiting the diffusion of free radicals.

Scanning SAXS measurements were complemented by holographic imaging experiments. These distinct modalities can be seamlessly switched by slightly rearranging the setup, as depicted in Fig. 2.1. To this end, the sample was moved $30 \mathrm{~mm}$ downstream and the $\mathrm{KB}$ beam was coupled into the tapered channel of a two-dimensional waveguide (entrance and exit pupil $332.7 \mathrm{~nm} \times 142.9 \mathrm{~nm}$ and $154.1 \mathrm{~nm} \times 96.0 \mathrm{~nm}$, respectively) for coherence filtering. The waveguide fabrication and optics are detailed in [97]. After the alignment of the waveguide with a hexapod (SmarAct), a total photon flux of approximately $9.5 \times 10^{9}$ photons s $^{-1}$ was produced. The pixel detector was then replaced by a scientific CMOS (sCMOS) camera (Photonic Science, UK) with $6.5 \mu \mathrm{m}$ pixel size and pre-installed on the same detector bench. The sCMOS camera includes a gadolinium oxysulfide scintillator screen and a fiber optic coupling to the chip for photon conversion.

\subsection{Analysis of cellular diffraction patterns}

With a cleaned KB focused beam, scattering intensity can be recorded at high signal-to-noise ratio. In this experiment, the azimuthally averaged signal extended up to a resolution limited by the angular acceptance of the detector at $q_{r}^{\max }=$ $0.57 \mathrm{~nm}^{-1}$. The radial momentum transfer is defined as $q_{r}=\left(q_{y}^{2}+q_{z}^{2}\right)^{1 / 2}=$ $(4 \pi / \lambda) \sin \theta$, where $2 \theta$ is the scattering angle between the primary and scattered 
beam and $\lambda$ the radiation wavelength. Towards low spatial frequencies, the signal was limited to $q_{r}^{\min }=0.078 \mathrm{~nm}^{-1}$ owing to the beamstop, whose size was dictated by the requirement to remove residual tails of the KB beam not removed by the soft-edge apertures.

Structural parameters that can be determined from the local diffraction patterns are, for example, (i) transmission as a measure of the projected absorption coefficient, (ii) center of mass of the beam (without beamstop) as a measure of the phase shift and hence the gradient of the projected electron density, (iii) the integrated intensity outside the beamstop, the so-called dark field contrast, as a measure of the overall density of scattering units, (iv) the local anisotropy and hence orientation of the dominating scattering units, and (v) model-based structural parameters obtained from least-squares fitting of the diffraction pattern [44, 98, 99].

Fig. 2.2(a) shows one example of a SAXS map (dark field contrast, $500 \mathrm{~nm}$ step size) of freeze-dried cardiomyocytes along with a fluorescence micrograph (Fig. $2.2 \mathrm{~b})$ recorded after freeze-drying. Inspection of the local diffraction data reveals a striking diversity of diffraction patterns. For illustration, Fig. 2.2(c) shows isolated diffraction patterns at eight selected positions to visualize the variation, ranging from isotropic signals to highly anisotropic modulated signals.

As a first approach, we have adopted a model-independent analysis and quantification of scattering intensity by means of principal component analysis (PCA). Following the procedure reported in [44], the orientation and anisotropy of each diffraction pattern can be computed by batch processing the data. Briefly, the covariance matrix of the diffraction pattern is evaluated and the eigenvalues $\lambda_{1}$ and $\lambda_{2}$, corresponding to the first and second principal (orthogonal) axis, respectively, are used to define a local anisotropy parameter $\omega=\left|\lambda_{1}-\lambda_{2}\right| /\left(\lambda_{1}+\lambda_{2}\right)$. The color-coded anisotropy parameter is shown in Fig. 2.3(a), superimposed by a solid black line indicating the orientation of the first principal component rotated by $90^{\circ}$ to reflect the structural orientation in real space. For displaying purposes, no line is drawn if $\omega$ was below a threshold set to 0.1 .

Comparison of the fluorescence image (Fig. 2.3b) and PCA (Figs. 2.3a and 2.3c) suggests that the anisotropy can in part be linked to the presence of actin fiber bundles. However, not all actin fiber bundles which can be clearly identified in the fluorescence micrograph appear in the anisotropy map: see for example the zoom regions in Fig. 2.3(c). Furthermore, high eigenvalues also occur in the denser nucleus in the absence of actin. Near the cellular boundary, where modulations of the streak-like patterns are also encountered, the anisotropy can be very pronounced: see for example in Fig. 2.3(c,1). This could possibly be caused by strong filament 

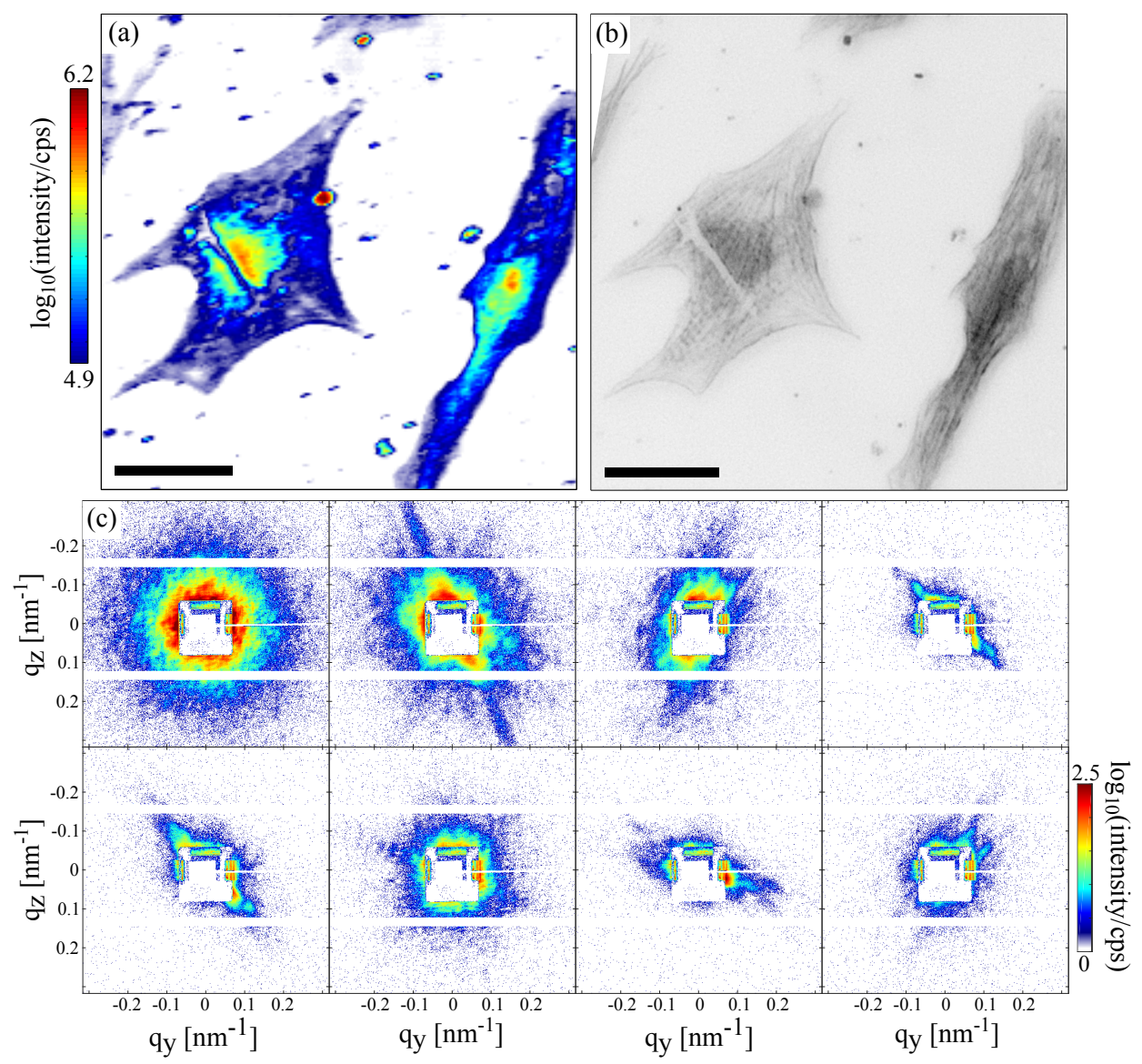

Figure 2.2.: Representative scanning X-ray diffraction dataset of single cardiomyocytes (freeze-dried state). (a) SAXS image (dark field) with $500 \mathrm{~nm}$ step size. Scale bar: $25 \mu \mathrm{m}$. (b) Optical fluorescence image of the labeled actin network recorded after freeze-drying. Scale bar: $25 \mu \mathrm{m}$. (c) Single exposures at different locations illustrate the diversity of local diffraction patterns, in particular in view of the anisotropy. 


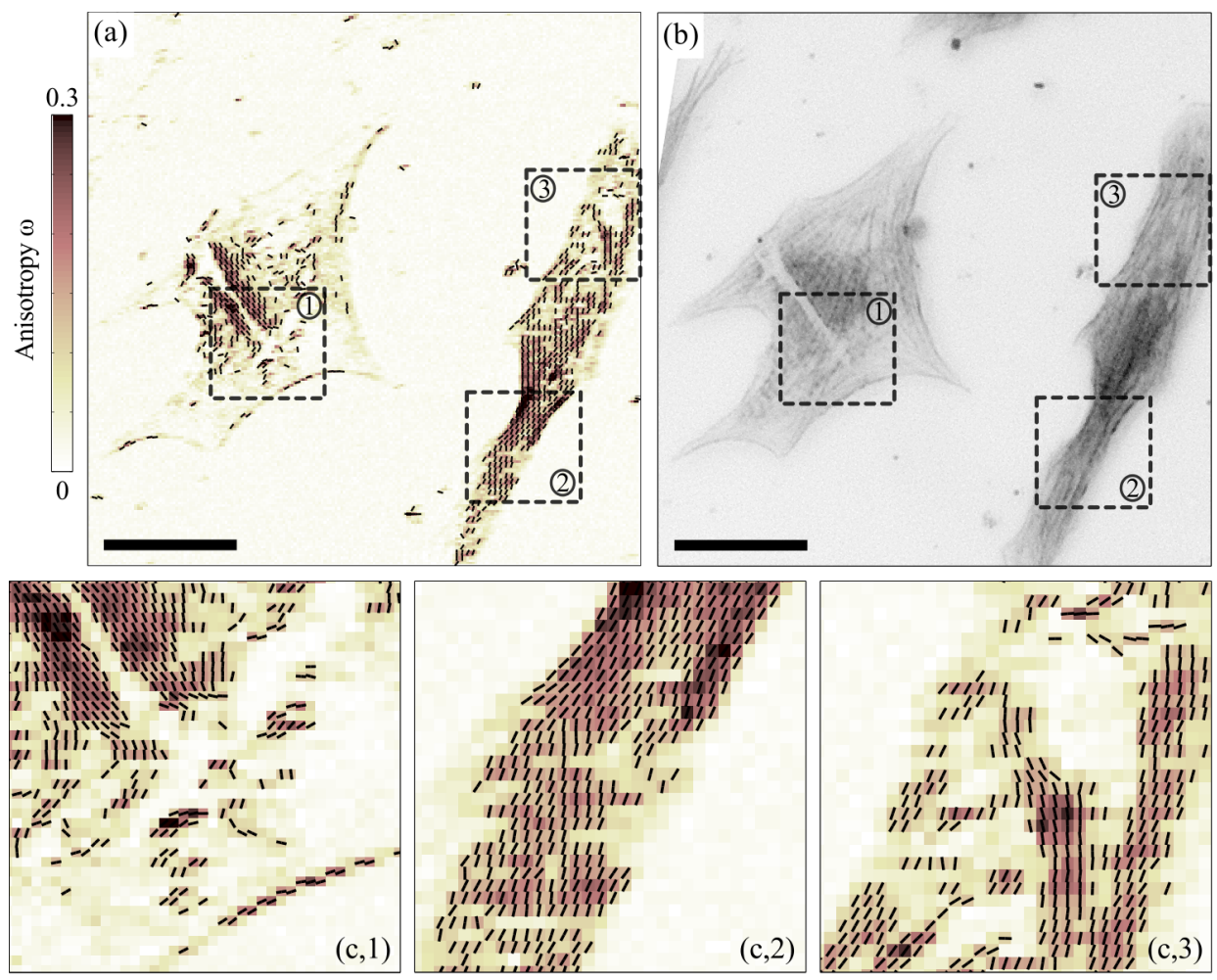

Figure 2.3.: Principal component analysis of the dataset presented in Fig. 2.2. (a) Colorcoded anisotropy parameter $\omega$. Structural orientation is marked by a solid black line indicating the fiber bundle orientation. Scale bar: $25 \mu \mathrm{m}$. (b) Fluorescence microscopy image as in Fig. 2.2. Scale bar: 25 um. (c) Enlarged regions of interest. Large eigenvalues are obtained around the nucleus and along the border of the cell, while small values occur in the cellular matrix. Notably, the anisotropy is most pronounced in $(c, 2)$ where the fluorescence image clearly shows high filament and bundle concentration and collective orientation.

bundling. Contrarily, the cellular matrix is characterized by lower eigenvalues.

Next, in order to determine the one-dimensional SAXS structure factors for three distinct cellular regions, marked in Fig. 2.4(a), diffraction data were averaged seperately for each cellular (a, b, c) and background (d) region. Then, background was subtracted from the mean scattering intensity of regions $\mathbf{a}, \mathbf{b}$ and $\mathbf{c}$ to yield three averaged and background-corrected scattering patterns. Prior to azimuthal integration, some data points, corresponding to detector gaps and beam stops, are discarded (masking). To account for anisotropy in the scattering signal, present for example in region c, we have further reduced the number of data points to a 

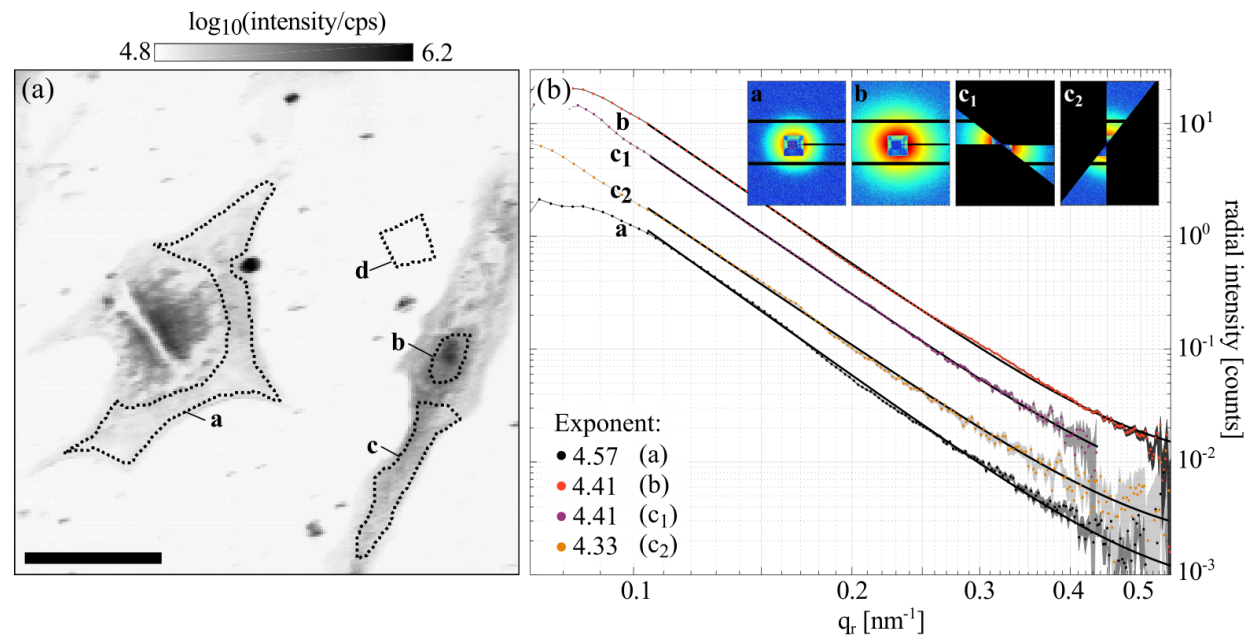

Figure 2.4.: One-dimensional structure factor obtained from multiple regions of interest. (a) Dark field image with $500 \mathrm{~nm}$ step size. Four regions of interest were defined (a: cell periphery, b: nucleus, $\boldsymbol{c}$ : cell periphery, $\boldsymbol{d}$ : background). Scattering data were averaged within each region seperately. The mean scattering intensity from region $\boldsymbol{d}$ was used for background subtraction. Scale bar: $25 \mu \mathrm{m}$. (b) Fit of the azimuthally averaged diffraction patterns (solid dots) to a power-law decay with a linear background (solid black lines). Data points corresponding to modular gaps and beam stops (marked black, see inset) have been discarded before the angular averaging process. $c_{1}$ and $c_{2}$ represent data obtained from a single averaged diffraction pattern, azimuthally integrated in a $\pm 10^{\circ}$ wedge around the first and second principal axis, respectively. Error margins are indicated by a shaded background.

$\pm 10^{\circ}$ wedge around each principal axis (here denoted $c_{1}$ and $c_{2}$, respectively), $c f$. inset in Fig. 2.4(b). Following these pre-processing steps, data can be azimuthally averaged to yield one-dimensional intensity profiles as a function of $q_{r}$, i.e. the SAXS structure factor. Error margins are shown as shaded background for each graph assuming a Poisson error model for the raw diffraction data.

As expected, scattering from the nucleus is stronger than from the cellular periphery owing to an increased electron density.

The radial intensity profiles resulting from all of the regions, shown in Fig. 2.4(b), decay algebraically, as confirmed by the corresponding power-law fits $I\left(q_{r}\right)=$ $a q_{r}^{-b}+c$. We find $b>4$ and hence a steeper decay than the Porod exponent 4 , which would be characteristic of particles with smooth interfaces. 


\subsection{Holographic imaging}

Next, we exploit the fact that the focusing setup used for scanning SAXS is compatible with holographic full-field imaging [63]. Holographic projections can be acquired by rearranging the experimental setup as described above. The acquired in-line holograms are processed numerically by phase retrieval algorithms to yield two-dimensional maps of the projected electron density.

As a first approach, we used the modified iterative hybrid-input-output (mHIO) algorithm [80] for single-distance phase retrieval based on the support constraint. For comparison, direct reconstructions based on the contrast transfer function (CTF) were performed [100]. The phase maps retrieved from such direct reconstructions could be directly used as a highly accurate support definition required for subsequent iterative phase reconstruction.

Next, we extended the original mHIO algorithm [80] to data sets with multiple propagation distances. Multiple distances are typically employed in CTF-based reconstructions to avoid missing information as a result of the zero points in the CTF. While the mHIO algorithm was originally designed for single-distance phase retrieval of pure phase objects [80], the multi-distance extension can phase also mixed objects by employing longitudinal diversity. In addition, the signal-to-noise ratio and convergence speed increases, and residual low-frequency components after background subtraction are less detrimental for image quality [65]. The extension to multiple distances is implemented such that the iteration result from one distance is used to initialize the next iteration for the consecutive distance, looping sequentially over all distances. The support constraint is kept constant during this procedure. One iteration round is completed after sequentially performing a single iteration for all distances.

A series of holograms of the sample was acquired at five defocus positions, $x_{1}=$ 29.6, 30.1,31.6, 33.6 and $38.6 \mathrm{~mm}$, with respect to the focus position at $x_{1}=0 \mathrm{~mm}$ and divided by their respective empty image. A stack of five holograms is shown as an example in Fig. 2.5(a) (front view: hologram recorded at distance $x_{1}$ ). Misalignment due to lateral drift was compensated for by sub-pixel image registration on trial reconstructions using the CTF reconstruction approach, as for example demonstrated in [101]. Rescaled and aligned projections could then be used for direct and iterative phase retrieval. All relevant experimental parameters are summarized in Tab. 2.1.

Fig. 2.5(b) depicts an mHIO reconstruction from an entire set of projections after 637 iterations of the algorithm. The phase map clearly depicts the internal 
structure of the cell, primarily the dense actin network, cell nucleus and smaller organellar structure.

Next, we compare mHIO reconstructions from a single and multiple distances with the corresponding CTF-based reconstructions. This is shown in Fig. 2.5(c) for a representative zoom region indicated by the dashed black frame in Fig. 2.5(b). The entirely different and independent reconstructions are highly consistent, proving the validity of the phase retrieval step. Despite the general agreement, however, there are notable differences in image quality.

CTF-based reconstructions from single and multiple distances both show lighter, almost bright, regions around the nucleus. Such halo-like artifacts result from erroneous constraints of the low frequencies in the CTF that are required to suppress the effects of low-frequency background variations. Contrarily, no such artifacts are observed in the multi-distance mHIO. The azimuthally averaged power spectral density (PSD) in Fig. 2.5(d) also indicates a higher capability of the mHIO to cope with artifacts from the CTF zero crossings. All curves reach the noise plateau at about 0.2 cycles per second, corresponding to a resolution of $94 \mathrm{~nm}$, at an effective pixel size of $37.7 \mathrm{~nm}$ given by the magnification of the system.

The differences in the reconstructions shown in Fig. 2.5(c) can therefore be summarized as follows: the input of five planes increases the image quality over singleplane reconstructions in particular for the CTF approach, while single-plane reconstructions from the $\mathrm{mHIO}$ algorithm are already of quite satisfactory quality and are only moderately improved upon further by taking data in five planes. The single-plane CTF has the lowest image quality of the four.

Lastly, we point out the complementary nature of the two experiments. With this aim, we show an azimuthally integrated PSD (mHIO, single distance) together with the structure factor obtained from the preceding section [graph b in Fig. 2.4(b)] in a single plot in Fig. 2.5(e). Omitting unphysical regions in the PSD and regions blocked by the beamstop in the SAXS experiment (black dots), it becomes apparent that the two modalities are complementary with regard to the window covered in momentum transfer. Two dashed black lines following a power-law decay with Porod exponent 4 were added to serve as a guide to the eye and to underline the overall Porod-law decay. In combination, the two techniques cover up to three orders of magnitude in momentum transfer.

The combination of the two techniques brings about the possibility to use holographic recordings to monitor radiation-induced structural alterations in scanning SAXS recordings.

To this end, we have recorded an X-ray hologram of an isolated cell before and 

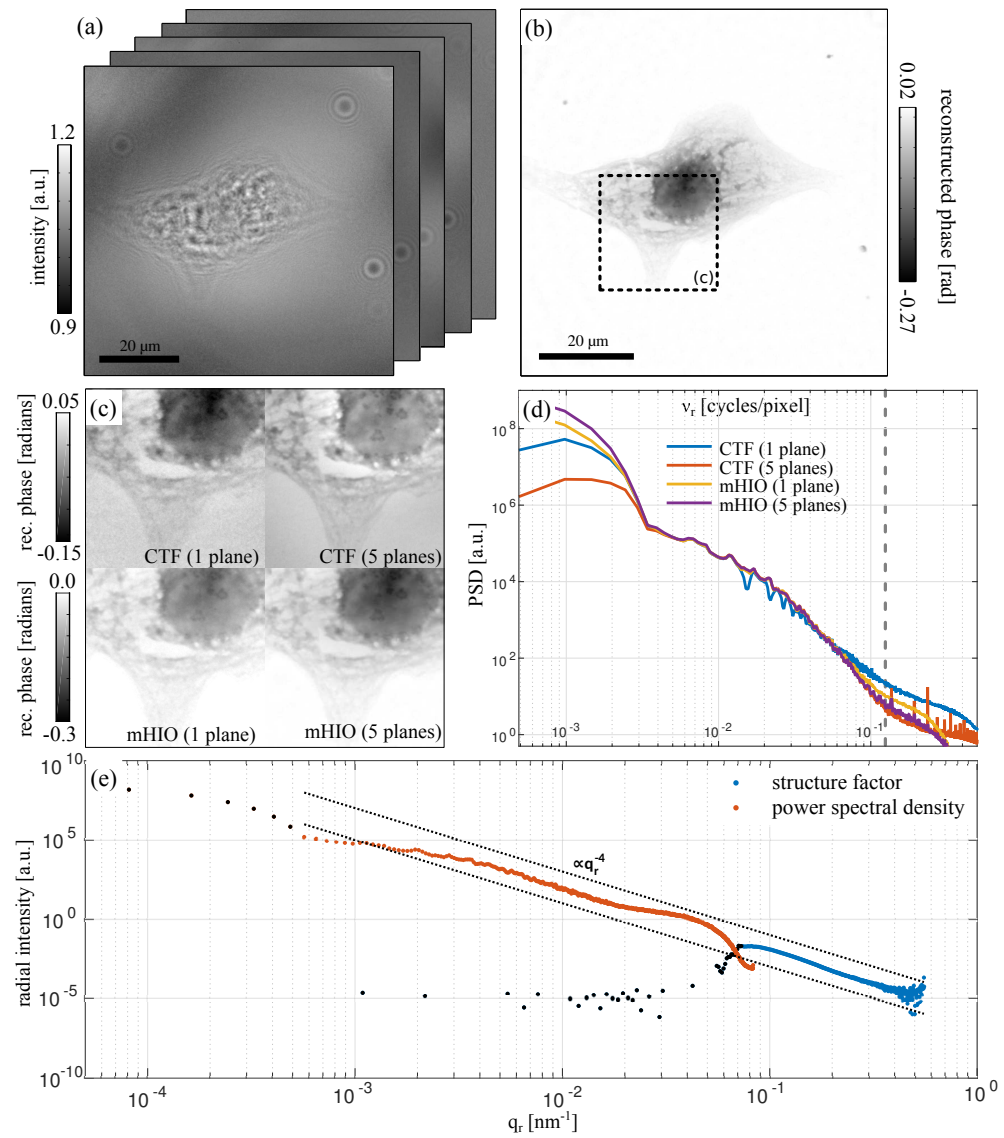

Figure 2.5.: Phase reconstruction of a single cardiomyocyte from holographic projections recorded at five defocus positions reconstructed directly on the basis of the contrast transfer function and iteratively using the mHIO algorithm. (a) Holograms recorded at five defocus positions (front view: hologram recorded at defocus distance $x_{1}$ ). Because of fluctuations in the beam profile, low-frequency components remain visible after background correction. (b) Reconstructed phase (or projected electron density) recovered from five holographic projections using the mHIO iterative algorithm. A dashed black frame marks the zoom region for direct comparison of the CTF and mHIO approaches in (c). (c) $\mathrm{CTF}$ and $\mathrm{mHIO}$ reconstructions from single and multiple distances for visual comparison. Corresponding power spectral densities are shown in (d). Single-distance CTF exhibits pronounced minima due to zero crossings in the contrast transfer function. The transition to the noise plateau occurs in all cases at around $94 \mathrm{~nm}$, as indicated by the dashed gray line. For comparison, (e) shows the PSD (single-plane mHIO) along with the structure factor (Fig. 2.4, graph b). Two dashed black lines, representing a power-law decay proportional to $q_{r}^{-4}$, serve as guide to the eye to underline the overall Porod-law decay. Note that intensity values have been scaled against each other. 


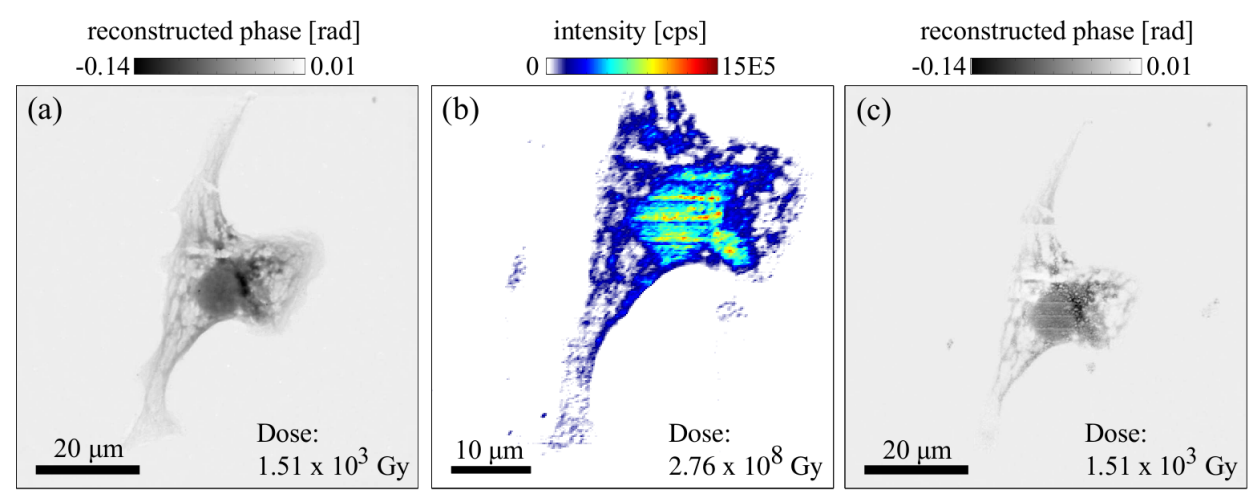

Figure 2.6.: Reconstructed (projected) electron density from holograms taken before and after a SAXS scan (background subtracted) on a single cardiomyocyte. (a) Reconstructed phase recovered from five holograms taken at defocus distance $x_{1}=29.6,30.1,31.6,33.6$ and $38.6 \mathrm{~mm}$ using the $\mathrm{mHIO}$ phase retrieval algorithm. (b) Scanning SAXS dataset. Absorbed dose includes exposure from two previous coarse 'search scans', visible as horizontal stripes along the sample. (c) Reconstructed phase from a second holographic recording with identical parameters to those in (a), acquired after the SAXS scan.

after the collection of scanning diffraction data, checking for structural alterations caused by radiation damage, as illustrated in Fig. 2.6. Fig. 2.6(a) shows the holographic reconstruction of a cardiac muscle cell prior to SAXS scanning. The consecutive SAXS scan (background subtracted) is shown in Fig. 2.6(b). Furthermore, two coarse scans, necessary to relocate the exact position of the cell, had already clearly damaged the cellular structure before the high-resolution dataset shown in (b) was recorded. These 'search scans' are also visible in the holographic reconstruction in (c). Comparing Figs. 2.6(a) and 2.6(c) it becomes obvious that a single SAXS scan alters the structure and ultimately destroys the sample under investigation, unless the dose is stringently controlled by reducing dwell time and by use of attenuators. Estimates for the dose $D$ given in Fig. 2.6 are calculated from

$$
D=\frac{I_{0} \tau E_{\mathrm{ph}}}{d \rho \Delta_{y} \Delta_{z}},
$$

given the total photon flux $I_{0}$, photon energy $E_{\mathrm{ph}}$, illuminated area $\Delta_{y} \Delta_{z}$ and exposure time $\tau$ as summarized in Tab. 2.2. The mass density $\rho$ and corresponding attenuation length $d$ are commonly estimated for cellular samples to be $\rho=$ $1.35 \mathrm{~g} \mathrm{~cm}^{-3}$ and $d=7.5 \times 10^{-4} \mathrm{~m}[102,103]$. 


\subsection{Summary, conclusion and outlook}

In summary, we have shown that scanning SAXS can be advantageously combined with full-field holography. This provides an approach to control structural degradation and beam damage online during the experiment and a means for locating sample features of interest. Importantly, full-field X-ray imaging is highly suitable to support scanning SAXS experiments by enlarging the covered length scales by two orders of magnitude. In future, this may even be extended to study timeresolved structural rearrangement during contraction of single muscle cells. Owing to the abrupt nature of the contraction process, holographic movies would have to be recorded at sampling rates exceeding ten frames per second while diffraction experiments would require a strategy of targeting isolated sampling points in order to circumvent beam damage.

Indeed, the present results show that each scan point in the scanning SAXS recordings should only be illuminated for a single time, and that at the given settings with intense KB-focused radiation only sub-second dwell times are admissible before a rapid decrease in scattering intensity is observed. Data acquired under such conditions and in particular without extensive 'search scans', however, show high consistency with visible light fluoresence microscopy, as judged by comparison of actin filament orientation and the SAXS anisotropy as well as the general shape and outline of the cells.

While the striation pattern observed in the fluorescence images of the cardiomyocytes indicated higher-order sarcomeric structures, we did not find any such indications of ordered actomyosin complexes in the X-ray structure factor. This can in part be explained by the limited accessible $q$ range due to the excessively large beamstop required to suppress the tails of the KB beam and the limited field of view of the pixel detector. Hence, in order to fully profit from the high efficiency of the KB focusing system, a larger detector must be used in future. However, since the accessible $q$ range would still allow for the detection of diffraction peaks known from muscle diffraction experiments, we point out that the lack of the typical layer line intereference maxima is indeed an interesting observation. In view of the sarcomeric structure, an extension of this work will consider three different solutions to the problem of missing data at low $q$ : (i) a cleaner KB probe by way of optimizing slit settings and apertures in front of the focal plane, (ii) alternative focusing optics such as compound refractive lenses offering a more Gaussian-like probe, and (iii) combination of micro- and nanofocused beams in scanning SAXS studies to extend the resolution in real and reciprocal space simultaneously. Finally, suitable 
Table 2.1.: Summary of experimental parameters for the aquisition of a defocus series as described in Sec. 2.4.

For single-distance recordings, the sample was placed at position 1.

\begin{tabular}{llllll}
\hline Defocus position & 1 & 2 & 3 & 4 & 5 \\
\hline Defocus distance $(\mathrm{mm})$ & 29.6 & 30.1 & 31.6 & 33.6 & 38.6 \\
Magnification ratio & 173.1 & 170.3 & 162.2 & 152.5 & 132.8 \\
Effective pixel size (nm) & 37.78 & 38.41 & 40.33 & 42.88 & 49.26 \\
Beam divergence (mrad) & \multicolumn{5}{c}{5.1} \\
Focus-detector distance (m) & \multicolumn{5}{c}{$2048 \times 2048$} \\
Pixel (horizontal $\times$ vertical) & \multicolumn{5}{c}{6.54} \\
Pixel size $(\mu \mathrm{m})$ & & \\
\hline
\end{tabular}

Table 2.2.: Experimental parameters for dose calculation.

In the case of Scan 4, the scan time for each defocal position is accumulated. Scan 1 and 2 are referred to as 'search scans' in the manuscript.

\begin{tabular}{lllllll}
\hline Scan & Energy $(\mathrm{keV})$ & $\mathrm{I}_{0}$ (counts per second) & $\Delta_{y} \times \Delta_{z}\left(\mu \mathrm{m}^{2}\right)$ & $\mathrm{N}_{y} \times \mathrm{N}_{z}$ & $\mathrm{~T}(\mathrm{~s})$ & Dose $(\mathrm{Gy})$ \\
\hline 1 (scanning SAXS) & 8.0 & $8.86 \cdot 10^{10}$ & $2.5 \times 2.5$ & $21 \times 21$ & 0.1 & $1.74 \cdot 10^{6}$ \\
2 (scanning SAXS) & 8.0 & $8.86 \cdot 10^{10}$ & $2.5 \times 2.5$ & $21 \times 21$ & 0.1 & $1.74 \cdot 10^{6}$ \\
3 (scanning SAXS) & 8.0 & $8.86 \cdot 10^{10}$ & $0.2 \times 0.2$ & $251 \times 251$ & 0.1 & $2.72 \cdot 10^{8}$ \\
4 (holography) & 8.0 & $0.57 \cdot 10^{9}$ & 11587 & $1 \times 1$ & 25.0 & $1.51 \cdot 10^{3}$ \\
\hline
\end{tabular}

models must be developed to access the full information of the local SAXS curves, including comparisons with pure actin assemblies.

\section{Acknowledgements}

We thank several members of our group, in particular Mareike Töpperwien, Aike Ruhlandt, Marius Priebe and Markus Osterhoff for the support during beamtimes and Susanne Hengst for her expertise and support in cell culture. We further acknowledge Michael Sprung and his team at DESY for excellent conditions of the P10 beamline and instrumentation. We thank Tina Althaus and Marion Kunze (Max-Planck-Institute for Dynamics and Self-Organization, Göttingen) for preparing cardiomyocytes from neo-natal rat and for further help and advice on cell preparation. Funding by the Sonderforschungsbereich 937 Collective behavior of soft and biological matter/project A11 by the German science foundation (DFG) is gratefully acknowledged. 


\title{
3. Scanning X-ray diffraction on cardiac tissue: automatized data analysis and processing
}

\author{
J.-D. Nicolas, M. Bernhardt, A. Markus, F. Alves, M. Burghammer and T. Salditt \\ Reproduced from Journal of Synchrotron Radiation 24, 1163 - 1172 (2017).
}

A scanning X-ray diffraction study of cardiac tissue has been performed, covering the entire cross section of a mouse heart slice. To this end, moderate focusing by compound refractive lenses to micrometer spot size, continuous scanning, data acquisition by a fast single-photon-counting pixel detector, and fully automated analysis scripts have been combined. It is shown that a surprising amount of structural data can be harvested from such a scan, evaluating the local scattering intensity, interfilament spacing of the muscle tissue, the filament orientation, and the degree of anisotropy. The workflow of data analysis is described and a data analysis toolbox with example data for general use is provided. Since many cardiomyopathies rely on the structural integrity of the sarcomere, the contractile unit of cardiac muscle cells, the present study can be easily extended to characterize tissue from a diseased heart.

\subsection{Introduction}

Classical small-angle X-ray scattering (SAXS) and X-ray diffraction (XRD) studies are well established tools for the characterization of biomolecular matter and biomaterials. High signal levels are achieved in particular with synchrotron radiation, but still largely rely on macroscopic ensemble-averaging. Local distributions of structural properties cannot be accessed with such an approach. For many samples, however, global average in real space leads to a signal, which is difficult to interpret and to model. In practice, this entails a severe restriction of diffraction methods to homogeneous model systems in many biophysical studies. For the 
case of biological tissues, for example, biological function relies on both a specific and well defined molecular nano-structure as well as an equally well defined cyto-architecture on the micrometer scale. Ideally, both scales should be probed simultaneously. Heart tissue underlying the physiological function of contractility is an excellent example. Classical diffraction studies on muscle (both for skeletal and cardiac muscle) have contributed to the understanding of the sarcomere as the basic structural unit of the myofibrils which make up muscle fibers. However, it is largely unknown how the molecular structure parameters vary within the tissue, in terms of local orientation, strain and variation in filament lattice spacing, and how they correlate to the textures visualized by histological microscopy.

To avoid the loss of information associated with averaging the signal over many heterogeneous areas, samples can be raster scanned with focused synchrotron beams [104-108]. However, decreasing the illuminated diffraction volume in each exposure results in a drastic increase in dose or decrease of the signal (or a combination of both). This has been a key challenge in developing X-ray experiments with microor nano-focused X-ray probes [109], in particular for soft biological samples.

The optics itself required for scanning diffraction experiments has undergone significant progress in recent years. Due to major improvements in the fabrication of focusing optics for hard X-rays, micro- and nanometer focal spot sizes can now be routinely achieved [110-112]. Recent developments in X-ray focusing optics have, for example, been review in [113]. Furthermore, signal-to-noise levels in experiments employing tightly focused beams have improved with the development of novel detection mechanisms for the recording of two-dimensional diffraction patterns $[114,115]$. Finally, accurate sample positioning has been implemented at many synchrotron instruments to generate maps of spectroscopic or scattering parameters, and are widely used for different techniques, for example in transmission X-ray microscopy, scanning X-ray fluorescence microscopy, scanning Bragg diffraction microscopy and X-ray ptychography. Scanning SAXS has also become a routine application, for example in the analysis of textured samples such as teeth [116-119], bone [32-37, 120, 121], wood [28-31], silk [122] and polymers [123]. Extensions to three-dimensional SAXS imaging include 3D-SAXS [38-40, 124, 125], SAXS-CT [47, 126] or energy-resolving SAXS [127]. However, as the experiments impose a significant radiation dose onto the sample, scanning SAXS and scanning X-ray diffraction have been largely restricted to strongly scattering materials (where the beam can be attenuated) or to materials which are comparatively radiation insensitive, such as bone for example.

In this work, we show that by combination of moderate focusing, fast scanning, 
sufficient distance between the illuminated points, background reduction and optimized detection, scanning SAXS and scanning X-ray diffraction become compatible with soft radiation-sensitive biological tissues. As an example and proofof-concept, we describe scanning X-ray diffraction experiments performed on an entire cardiac tissue slice. With scanning strategy and instrumentation cleverly chosen, meaningful signals can be recorded, and high data throughput automatized analysis becomes the main challenge. To this end, we have developed a versatile data-analysis platform to extract multiple order parameters from the scattering data of tissue. We can thus add real space information to classical SAXS and diffraction studies on cardiac tissue [25, 128] by tracing order parameters related to the sarcomere structure throughout an entire slice of cardiac tissue.

The article is outlined as follows. Firstly, in Sec. 3.2 the scanning X-ray diffraction experiment on a cardiac tissue slice is described and results are discussed. Next, in Sec. 3.3, methods for automated data treatment in scanning diffraction experiments are presented. The article closes with a summary and conclusion in Sec. 3.4. An example dataset and a description of the general layout of the data analysis platform is given in Appendix A. Data analysis software written in the programming language $M A T L A B$ (Mathworks Inc., USA) is available online to be used by the scientific community.

\subsection{Scanning $\mathrm{X}$-ray diffraction on cardiac tissue}

\subsubsection{Experiment}

Data on cardiac tissue was collected at the ID13 endstation (microbeam hutch) of the European Synchrotron Radiation Facility (ESRF, Grenoble, France). The workflow of the scanning diffraction experiment is shown in Fig. 3.1. Cardiac tissue was obtained from an excised mouse heart. After excision, the heart was embedded in agarose [4\% in phosphate-buffered saline (PBS)] and cut in a vibratome (VT1000 S, Leica Biosystems, Germany) to a thickness of $30 \mu \mathrm{m}$. Prior to the experiment, the sample was mounted between two thin polypropylene foils and a small volume of PBS was added to prevent drying of the sample. The photon energy for the experiment was set to $13.45 \mathrm{keV}$ using a $\mathrm{Si}(111)$ channel-cut monochromator. Beam-defining slits and a compound refractive lens (CRL) transfocator were tuned to focus the beam to an elliptical spot size of $2 \mu \mathrm{m}(\mathrm{h}) \times 3 \mu \mathrm{m}(\mathrm{v})$. The total photon flux was $I_{0}=1.6 \times 10^{12}$ photons $^{-1}$. The sample was aligned using an on-axis video microscope that can be moved laterally with respect to the 


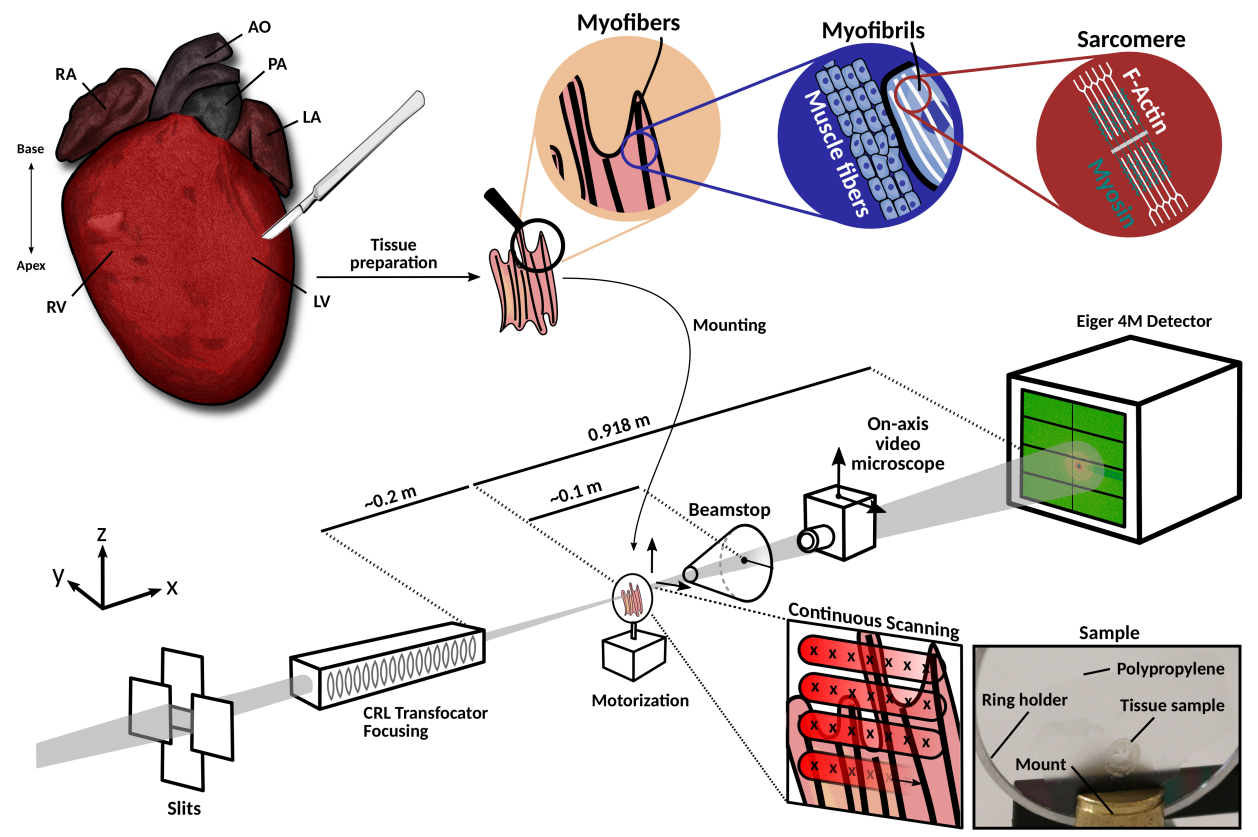

Figure 3.1.: Sketch of the scanning diffraction experiment on tissues. A $30 \mu \mathrm{m}$-thick tissue slice from an excised mouse heart, embedded in an agarose matrix, was mounted on a thin polypropylene foil and placed into the focus of the X-ray beam. Zoomed illustrations depict the highly regular arrangement of cardiac muscle fibers. Monochromated undulator radiation is focused down to micrometer spot size, using CRL transfocator optics. Scattered radiation is detected using a two-dimensional pixelated detector (here Eiger 4M, Dectris, Switzerland). An on-axis video microscope facilitates sample positioning and alignment with respect to the beam focus position. A circular beamstop housed in a helium-flushed flight tube blocks unscattered radiation downstream from the focus position. Finally, the sample is swept through the focus of the beam and diffraction patterns are recorded at regular intervals during scanning. The data are read and analyzed by a custom platform for automized data analysis for scanning diffraction experiments. $R A$, right artery; $L A$, left artery; $A O$, aorta; $P A$, pulmonary artery; $L V$, left ventricle; $R V$, right ventricle.

beam. A tailor-made beamstop housed in a flight tube flushed with helium was used at a distance of approximately $10 \mathrm{~cm}$ behind the sample stage to block the primary beam and to reduce background. The scattering patterns were recorded at equal time steps during scanning using an Eiger $4 \mathrm{M}$ detector, placed $918 \mathrm{~mm}$ downstream of the sample. Note that, in contrast to standard raster scanning of the sample through the beam focus, here the sample was moved continuously at a constant speed during data acquisition. This scheme of continuous scanning was indispensable to cover large tissue areas at tolerable scan time. 


\subsubsection{Results}

Since the goal of the present study was to image the entire cardiac tissue slice, a total sample area of approximately $6 \mathrm{~mm} \times 5 \mathrm{~mm}$ had to be covered. To this end, a total of $1090(\mathrm{~h}) \times 1331(\mathrm{v})$ scan points, an exposure time of $10 \mathrm{~ms}$ and a step size of $5 \mu \mathrm{m}$ was chosen to image the entire tissue. Since the sample was illuminated continuously and moved at a constant speed, the step size is the distance covered between the start of adjacent exposures. In total, data collection took $8 \mathrm{~h}$ and $10 \mathrm{~min}$. With a $10 \mathrm{~ms}$ exposure time, this resulted in a scan overhead of approximately $10 \mathrm{~ms}$ per scan point.

Given the above parameters, an average dose of $577 \times 10^{3}$ Gy was recieved by the sample per scan point. The dose $D$ was calculated from [102]

$$
D=\frac{\mu I_{0} \tau E_{\mathrm{ph}}}{\rho \Delta x \Delta y},
$$

using tabulated values for the mass attenuation coefficient $\mu$ and mass density $\rho$, where $\mu / \rho=2.0641 \mathrm{~cm}^{2} \mathrm{~g}^{-1}[129] . \Delta x \Delta y=36.27 \mathrm{~mm}^{2}$ corresponds to the overall sample area scanned and $\tau=29400 \mathrm{~s}$ to the overall scan time during which the beam was impinging on the sample.

The overall data storage of this scan was $1.1 \mathrm{~TB}$ (using LZ4 compression). Scattered intensity and anisotropy was analyzed in parallel on a system using eight central processing units (Intel ${ }^{\circledR} \mathrm{Xeon}^{\circledR}$ CPU E5-2609, 2.40GHz; Intel Corporation, Santa Clara, USA). The overall computation time was $11 \mathrm{~h}$, largely limited by the process of file reading.

The result of the scan and an optical micrograph that has been recorded directly after the measurement is shown in Fig. 3.2. Based on the diffraction data, the anisotropy of the $(1,1)$ reflection as well as the myofibril orientation and the filament spacing of the acto-myosin lattice (Fig. 3.2c) were extracted by fully automatized scripts, as important parameters of cardiac muscle cyto-architecture. The filament spacing was determined from the maxima of the $(1,1)$ reflection in the structure factor $I\left(q_{r}\right)$, obtained by plotting the diffraction intensity as a function of radial momentum transfer $q_{r}=\left(q_{y}^{2}+q_{z}^{2}\right)^{1 / 2}=(4 \pi / \lambda) \sin (\theta)$, where $2 \theta$ is the scattering angle between the primary and scattered beam and $\lambda$ the radiation wavelength. Note that the interfilament spacing $d^{(1,0)}$ is obtained from the position of the $(1,1)$ equatorial reflection by $d^{(1,0)}=\sqrt{3} 2 \pi / q_{r}^{(1,1)}$.

To extract anisotropy and orientation from the scattering distribution we have followed the approach described in [130]. In short, the anisotropy $\omega$ of the scattering 
distribution is defined as $\omega=\left|\lambda_{1}-\lambda_{2}\right| /\left(\lambda_{1}+\lambda_{2}\right)$, where $\lambda_{1}$ and $\lambda_{2}$ correspond to the eigenvalues of the covariance matrix of the momentum transfer along $q_{y}$ and $q_{z}$. The filament orientation in this model is defined as the angle of the largest eigenvalue rotated by $90^{\circ}$. To this end, the covariance matrix was determined within a detector region, defined by a suitable mask, in order to avoid contamination of the signal by background scattering close to the beamstop and by the noise floor at high $q_{r}$. As indicated in Fig. 3.5, values of $q_{\min }=0.22 \mathrm{~nm}^{-1}$ and $q_{\max }=0.38 \mathrm{~nm}^{-1}$ were found suitable. Myofibril orientation was color coded as presented in [131].

Given the myofibril orientation shown in Fig. 3.2(d), it becomes apparent that the myofibrils circulate around the left ventricle (LV) of the heart. The anisotropy of the scattering shown in Fig. 3.2(c) shows that the scattering is more isotropic in the bulk of the muscle. Interestingly, the position of the $(1,1)$ reflection is not constant throughout the tissue, but appears textured with reflection positions as large as $0.3 \mathrm{~nm}^{-1}$, which is significantly larger than the mean value $0.275 \mathrm{~nm}^{-1}$ of the present data. Furthermore, around the epi- and endocardium of the heart, the reflection position suddenly shifts to lower values of around 0.25 to $0.26 \mathrm{~nm}^{-1}$. The observed local changes in interfilament spacing might result from an interplay with the surrounding tissue matrix, and cannot be observed in macroscopic diffraction experiments averaging over large muscle regions. Note that the interfilament spacing is known to depend on many physiological parameters, in particular osmotic stress [132]. For a whole (unsliced), perfused and hydrated rat heart, the literature value of $q_{r}^{(1,1)}=0.29 \mathrm{~nm}^{-1}$ can be used as a reference [133].

More detailed views of the tissue within selected regions of interest (ROIs) are shown in Fig. 3.3. Based on the four ROIs marked in Fig. 3.2(b), each pixel in the ROIs has been analyzed with respect to the following four parameters: integrated scattered intensity, anisotropy, myofibril orientation and interfilament spacing. Some characteristic patterns of the tissue already become apparent in the darkfield maps, representing the local scattering strength of the tissue averaged over all $q_{r}$ values. From theses micrometer-sized structures, see for example the stripy pattern in ROI 1, preferential directions and tissue orientation be inferred. We stress, however, that the intrinsic orientation of biomolecular assemblies within the illuminated spot can only be assessed from analyzing the anisotropy. In the current case, this signal is dominated by myofibril orientation. Both the degree of orientation and the principal direction are quantified. Interesting myofibril arrangements can, for example, be observed in ROI 3. Finally, the results show that the interfilament spacing is not constant in all regions, but shows pronounced local variations. 

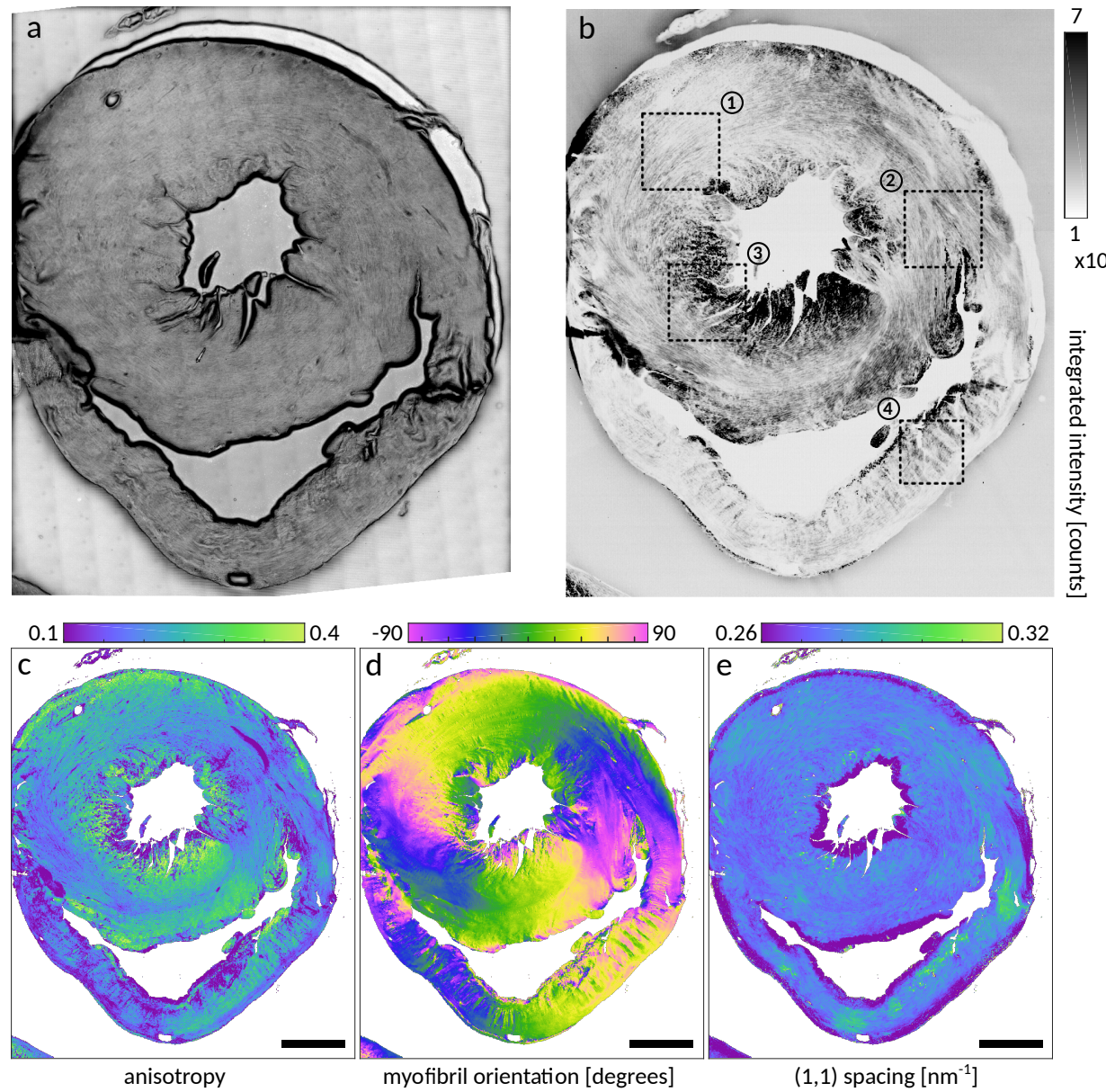

Figure 3.2.: Scanning $X$-ray diffraction dataset on tissue slices from mouse cardiac muscle. (a) Optical micrograph and scanning diffraction image (darkfield contrast) of a mouse heart. Multiple scattering parameters are extracted in a fully automated manner, such as (b) integrated scattered intensity, (c) anisotropy of the scattering resulting from the $d^{(1,1)}$ reflection from the acto-myosin lattice, (d) the corresponding myofibril orientation, and (e) the extracted mean position of the reflection along $q_{r}$. The mean positions were obtained by fitting a Gaussian and a background model to the structure factor $I\left(q_{r}\right)$. For clarity, background is plotted in white. 

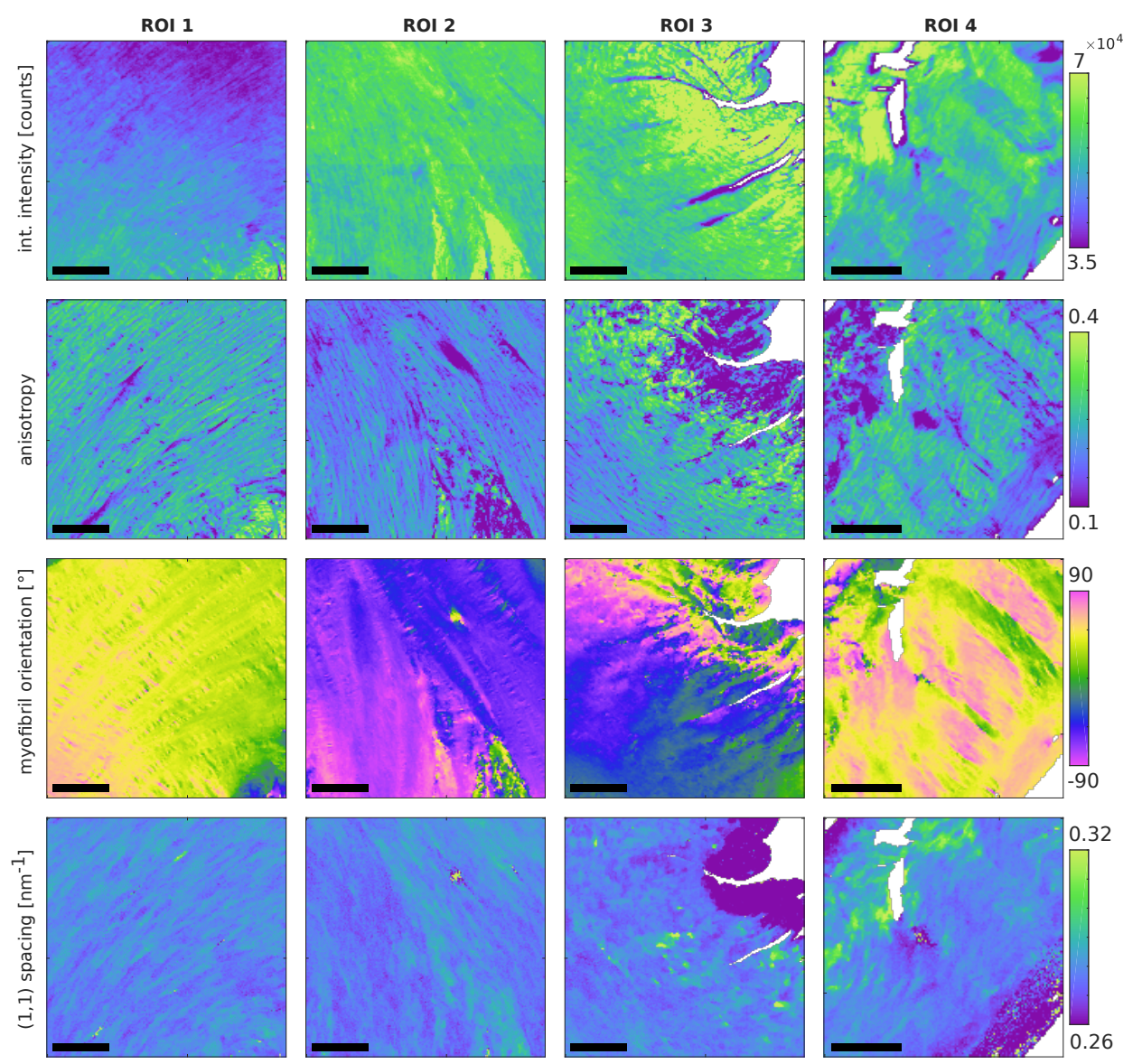

Figure 3.3.: Analysis of darkfield contrast, anisotropy, myofibril orientation and actomyosin lattice spacing, as in Fig. 3.2. Regions of interest (ROIs) are marked by black dashed boxes in the overview darkfield map in Fig. 3.2(b). Scale bar: $100 \mu \mathrm{m}$.

The interfilament spacing is deduced pixel-wise by performing an angular average of the data after background subtraction. The data are fitted by a model function, consisting of two power law decays and a Gaussian distribution describing the $q_{r}^{(1,1)}$ reflection, i.e. $I\left(q_{r}\right)=a q_{r}^{-b}+c q_{r}^{-d}+e \exp \left[-\left(q_{r}-f\right)^{2} / \sigma^{2}\right]$, where $a-f$ are fit parameters. The reflection width was determined at $\sigma=0.034 \mathrm{~nm}^{-1}$ and fixed during the fitting process.

To verify, that indeed the reflection position is changing with lateral position within the sample, the angular averaged structure factor of three examplary data points A, B and $\mathrm{C}$ are depicted together with the respective model function in Figs. 3.4(a) and 3.4(b). In general, one could depict the distribution of each fitting parameter 

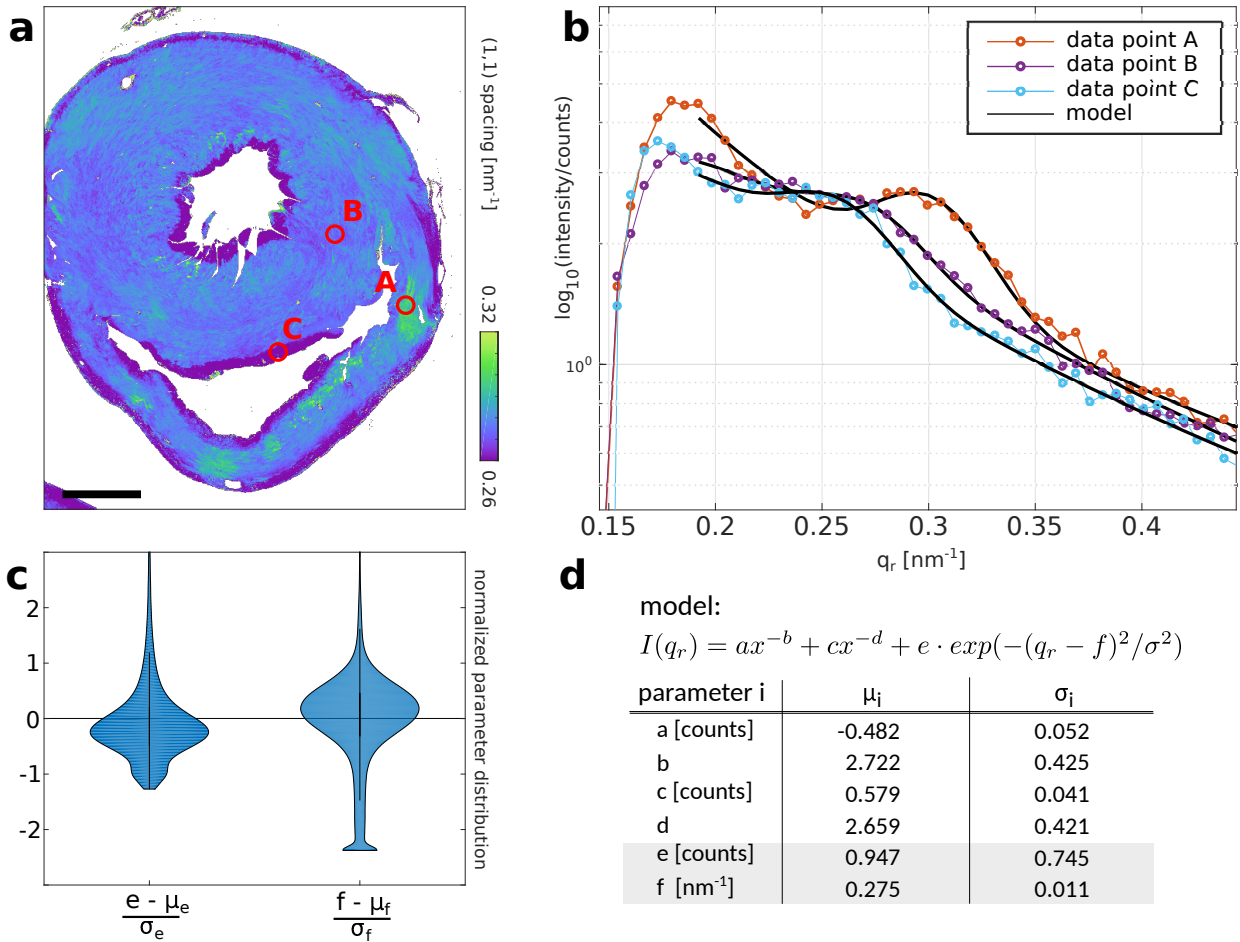

model:

$I\left(q_{r}\right)=a x^{-b}+c x^{-d}+e \cdot \exp \left(-\left(q_{r}-f\right)^{2} / \sigma^{2}\right)$

\begin{tabular}{l|c|c} 
parameter i & $\mu_{\mathrm{i}}$ & $\sigma_{\mathrm{i}}$ \\
\hline $\mathrm{a}$ [counts] & -0.482 & 0.052 \\
$\mathrm{~b}$ & 2.722 & 0.425 \\
$\mathrm{c}[$ counts] & 0.579 & 0.041 \\
$\mathrm{~d}$ & 2.659 & 0.421 \\
$\mathrm{e}[$ counts] & 0.947 & 0.745 \\
$\mathrm{f}\left[\mathrm{nm}^{-1}\right]$ & 0.275 & 0.011
\end{tabular}

Figure 3.4.: Comparison of scattering profiles and their respective fits from three selected data points on the sample. (a) Map of the $(1,1)$ lattice spacing as shown in Fig. 3.2. Red circles mark the location of the data points $A, B$ and $C$ for which azimuthally integrated structure factor profiles $I\left(q_{r}\right)$ are shown in (b) together with the respective fit. A fitting model composed of two power-law decays and a superimposed Gaussian was used to obtain the peak position $q^{(1,0)}$. (c) Violin plot of the normalized distributions of the reflection strength $I\left(q_{r}^{(1,0)}\right)$ (fit parameter e) and respective reflection position $q_{r}^{(1,0)}$ (fit parameter $f$ ). The arithmetic mean $\mu$ and standard deviation $\sigma$ of all fit parameters are summarized together with the model function in (d).

in its entirety. To this aim, all fit parameters have been normalized by subtraction of the arithmetic mean and dividing by the standard deviation $\sigma$ of the respective distribution. Fig. 3.4(c) visualizes the normalized distribution of the reflection strength (fit parameter $e$ ) and reflection position (fit parameter $f$ ). Mean and standard deviation of all distributions are tabulated for completeness in Fig. 3.4(d). 


\subsection{Processing and analysis of scanning diffraction data}

In the following section, we describe in more detail how scattering data from these scanning experiments were processed and analyzed in a robust, automated and model-independent fashion. Most of the steps a standard procedures, used in many diffraction experiments, but are described and illustrated for completeness. In particular, we want to stress that a research field which initially was started by analysis software, based on reading in a single diffraction pattern, treated by individual mouse clicks such as in [134], must now be replaced by fully automated and high-throughput scripts. All data processing tasks described here are implemented in the data analysis toolbox. Data analysis tasks not treated in this manuscript are documented in the toolbox.

One major step in data processing of two-dimensional diffraction data is a suitable data pre-processing. Many mistakes can be made, especially by an inexperienced user, in the data masking and data selection. Data masking is the task of identifying incorrect data, that will be neglected in further data processing tasks, while data selection reduces an otherwise valid data set to a desired range of $q_{r}$-values and angles, for example to discriminate a signal against background. An example of data masking and data selection is shown in Fig. 3.5. A raw diffraction pattern obtained using an Eiger 4M detector is shown in Fig. 3.5(a). Intermodular gaps and hot pixels are represented by $2^{16}$ counts. It is crucial to mask all invalid pixels by a detector mask depicted in Fig.3.5(b). Multiplying the raw detector image with the detector mask will result in an image shown in Fig. 3.5(c). Several analysis routines require data originating from within a certain $q_{r}$ range. A corresponding pixel mask that is later being used for data selection is shown in Fig. 3.5(d). Note that, in addition to defining logical data masks, data can also be selected based on identifying a certain region on the detector and binning the data by an integer fraction, as illustrated in Fig. 3.5(e) with a binning ratio of 4 .

Following data pre-processing steps such as masking, selection and binning, the two-dimensional data can be either directly analyzed or mapped to a one-dimensional representation. Common examples are azimuthal and radial integration of the data. Azimuthal integration refers to summing intensities over the azimuthal angle $\phi$ or a restricted (selectable) angular interval $\Delta \phi$, resulting in a one-dimensional $I\left(q_{r}\right)$ curve with selectable $\Delta q_{r}$ bins. If only a certain angular interval $\Delta \phi$ is evaluated, this is also commonly denoted as cake integration or wedge integration. Contrarily, radial integration refers to summation of intensities 

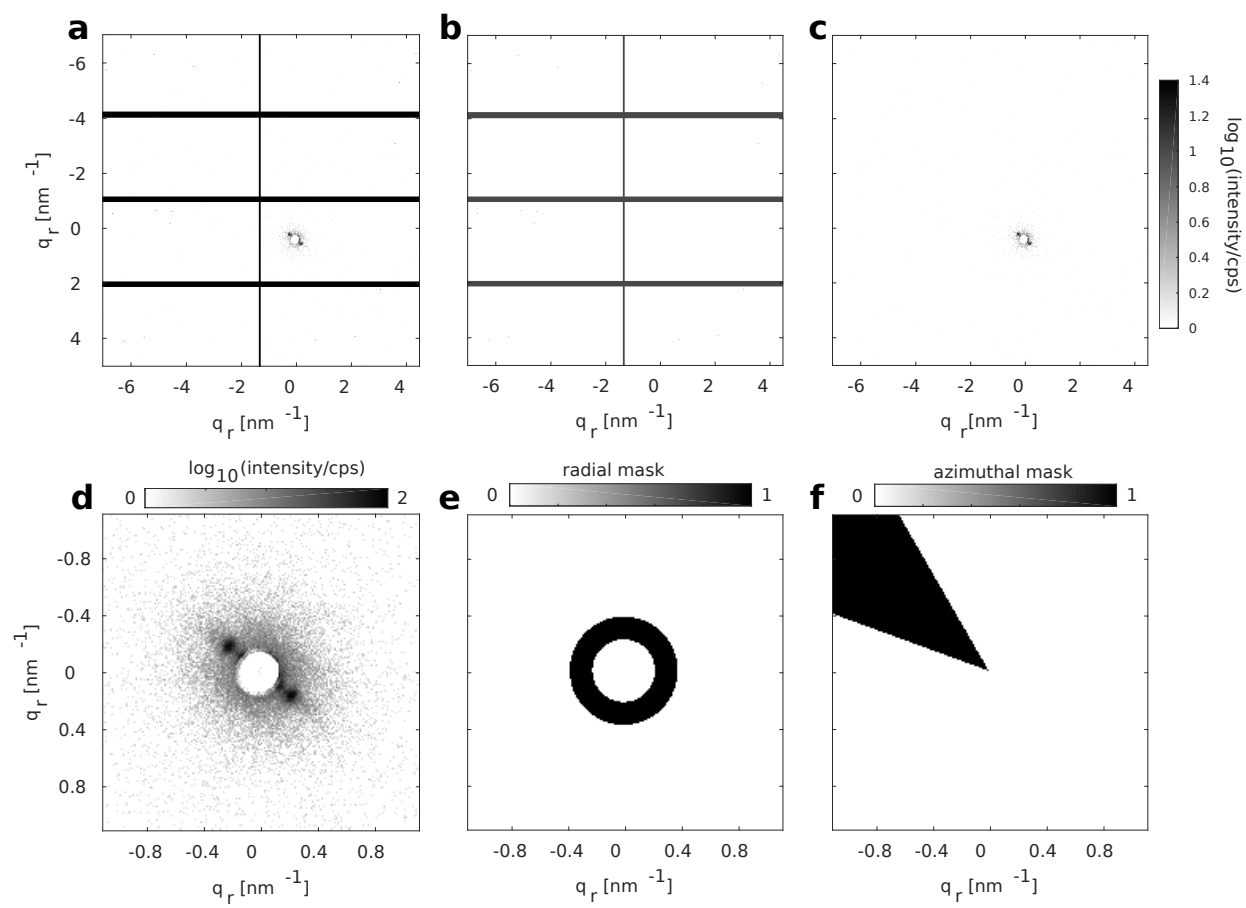

Figure 3.5.: Options for pre-processing of diffraction data. Shown in (a) is a raw detector image recorded with an Eiger $4 M$ detector. Modular gaps and hot pixels are represented by $2^{16}$ counts. By defining a logical mask for hot pixels, modular gaps and the beamstop area (b), the masked pixels are set to zero in the diffraction pattern (c). (d) In addition, ROIs can be defined and data can be binned by binning factors $i \in \mathbb{N}$ along each dimension (here, binning factor 2 along each dimension). (e) Radial mask defining a range from $0.22 \mathrm{~nm}^{-1}$ to $0.38 \mathrm{~nm}^{-1}$ and (f) azimuthal mask ranging from $120^{\circ}$ to $160^{\circ}$.

over the full radial range or a selected subset of the radial coordinate, yielding $I(\phi)$. The process of $2 d \rightarrow 1 d$ mapping is sketched in Fig. 3.6. For this purpose, different algorithmic implementations can be selected based on the users needs for accuracy, robustness or computational speed:

(i) A fast and straightforward approach for azimuthal integration simply rebins the two-dimensional data $I\left(q_{r}, \phi\right)$ in equidistant intervals $\Delta q_{r}$. Formally, this approach can be written as

$$
I(b)=\frac{\sum_{\left(q_{r}, \phi\right)} \Pi_{b}\left(q_{r}\right) I\left(q_{r}, \phi\right)}{\sum_{\left(q_{r}, \phi\right)} \Pi_{b}\left(q_{r}\right)},
$$

where

$$
\Pi_{b}\left(q_{r}\right)= \begin{cases}1 & \text { if } b \Delta q_{r} \leq q_{r}<(b+1) \Delta q_{r} \\ 0 & \text { otherwise }\end{cases}
$$



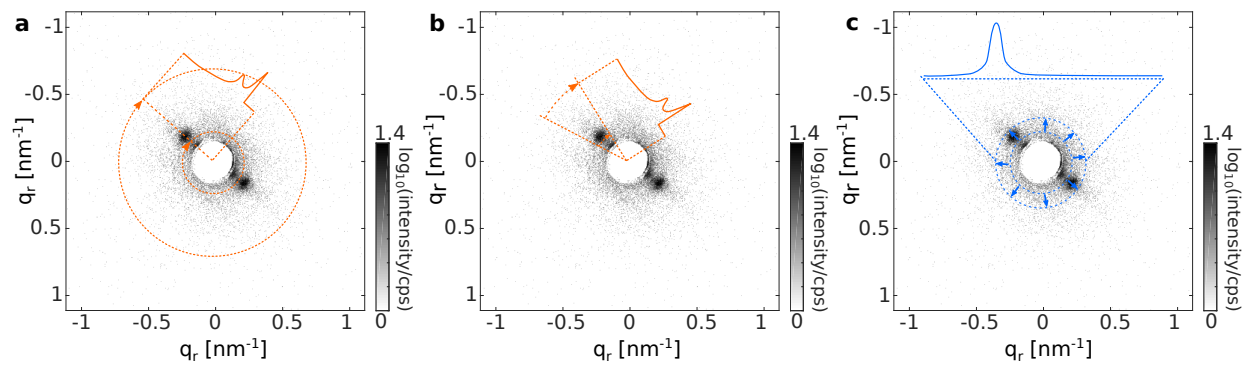

Figure 3.6.: Several $2 D \rightarrow 1 D$ mappings should be routinely feasible in scanning diffraction experiments. (a) Full azimuthal integration, (b) azimuthal integration within an angular range ('cake integration') and (c) radial integration within a certain $q_{r}$ range.

identifies all pixels corresponding to a given $q_{r}$ interval and $b$ is the corresponding integer bin index.

(ii) For more advanced data regridding, the $p y F A I$ suite [135] can be used. Since pyFAI is a computational library for azimuthal regridding written in the Python programming language, it is natively supported in the MATLAB code used here. In comparison, a simple azimuthal rebinning is faster than the basic azimuthal integration implementation (using the NumPy package for data processing). Since computational speed is of major concern, data regridding should be parallelized so that calculations can be distributed on multiple central processing units or the graphics processing unit.

\subsection{Summary and conclusion}

The experiment demonstrates that heart tissue slices can be mapped by scanning diffraction with high data throughput based on continuous scanning and fast pixel detector read-out. In particular, the entire (small animal) heart cross section can be covered in one scan, at still sufficiently fine (micrometer-sized) sampling. Plotting the result of such a diffraction scan, the signal level of the X-ray scattering provides an X-ray darkfield contrast for the tissue, which already reflects the cyto-architecture of the tissue slices quite well. By automated analysis of the local diffraction pattern, we have further shown that the local filament orientation and lattice parameter, i.e. characteristic spacing of actin and myosin filaments in myofibrils, can be extracted by automated data analysis. Furthermore, not only the direction but also the degree of orientational order can be quantified. Scanning diffraction of muscle tissue is thus complementary to macroscopic muscle 
diffraction experiments which average over large ensembles, since they allow the biomolecular structure to be correlated with the local cyto-architecture. Interestingly, we have found pronounced local variations in the acto-myosin lattice parameter. Rather than strain in crystalline materials, where here such variations in lattice spacing reflect the local stress, such variations must occur in development (assembly) of tissue, and must be interpreted with respect to mechanical functions. In this way, we are convinced that the demonstrated approach can further advance cardiovascular research, which has taken ample advantage of synchrotron radiation [133, 136, 137]. However, only now has different technological progress enabled scanning and analysis of large tissue areas with micrometer-sized sampling, single-photon-counting pixel detectors and automated diffraction analysis.

At first sight, after more than two decades of progress in scanning micro- and nano-diffraction, the present scanning diffraction study of tissue may seem rather standard from a conceptional or even technical point of view. However, we must keep in mind, that concerning biological samples and in particular tissues, scanning diffraction is to date largely restricted to strongly scattering and in particular mineralized tissues, such as bone or wood, materials for which the technique was initially developed $[28,138,139]$. To date, much fewer studies on wet and soft tissues than on hard tissue have been published. This is for a good reason, as such experiments face two main challenges: firstly, low signal-to-noise due to a weak diffraction signal, and, secondly, an inherent sensitivity to radiation damage.

The first issue requires improvements of the optical scheme, involving optimized focusing and background reduction, but also of the analysis, since weak signals often necessitate careful pre-processing of the data and analysis schemes. At the same time, meaningful results often require larger scan areas to verify whether signals are representative and correlate with optical microscopy. While this is true for most samples, it becomes vital for weak signals, which are often more difficult to interpret in practice. As a result, high-throughput data recording and analysis is required. With progress in instrumentation (pixel detectors, beamline control and continuous scanning) and in analysis (automated scripts and algorithms, compatibility with large data volume), this has now become possible.

The second issue is more difficult. In the present example, the strategy was as simple as efficient: short exposure $(10 \mathrm{~ms})$, micrometer-sized focal spot $(2 \mu \mathrm{m}-3 \mu \mathrm{m})$ and a step size approximately two times larger than the spot size, so that always a fresh part of the sample was exposed. In other words, real-space resolution was deliberately sacrificed by choosing a micro-focused rather than a nano-focused beam. This has three advantages. Firstly, it helps to obtain a cleaner beam profile and 
lower background, resulting in a higher resolution in reciprocal space. Secondly, the lower flux density helps to avoid beam damage. Thirdly, larger volumes, in this case an entire slice of mouse heart, can be scanned. With the chosen parameters, the structural results are (at least not visibly) affected by radiation damage, and high throughput and sensitivity could be achieved for the important case of cardiac tissue. In principle, multiple sections of a mouse heart making up a full heart in three dimensions could be scanned by this approach. Furthermore, the present study can easily be performed on tissue from a diseased heart, since many cardiomyopathies have their origin in the sarcomere [140]. As an example, the interplay of the muscular tissue with the surrounding matrix, e.g. in cardiac fibrosis, can be studied.

One may be tempted to concede that cardiac tissue with its regular acto-myosin structure and parallel micro-filaments is more ordered than other tissues and may therefore be exceptionally amenable to diffraction studies, similar to biological materials such as wood and bone. However, many other tissues also exhibit high and interpretable signal levels, including for example the central and peripheral nervous system, related to the myelin sheath around axons. In the case of brain tissue, scanning diffraction might also be a valuable tool in the study of protein aggregations in neurodegenerative diseases. We are therefore convinced that the particular combination of fast scanning methods in combination with a fast acquisition scheme implemented now at many beamlines, as well as improvements in optics (focusing, background reduction) in combination with novel automated analysis tools, now make scanning diffraction applicable to soft biological tissues. The technique can complement standard histology and established microscopy techniques with additional contrast and structural information.

While the entire workflow from sample preparation and mounting to beam shaping, selection of scan parameters and detection scheme are equally important, we stress in this work in particular the role of automated analysis given the nowadays vastly increased amount of data generated during scanning diffraction experiments. To this end, we have presented a straightforward but numerically efficient approach. The custom-made data analysis platform for scanning diffraction experiments as well as an example data set have been made available online or can be obtained by the authors on demand. 


\section{Appendix A}

\section{Data analysis toolbox and example data}

\section{A1. Data analysis toolbox}

Data analysis software can often facilitate access to unexperienced users of an experimental technique. Small-angle X-ray scattering is a great example of how powerful analysis tools can increase accessibility and collaboration between research fields. In the case of scanning X-ray diffraction, much progress has been made in establishing software methods for X-ray data analysis. However, due to the diverse nature of the scattering signal to be expected from inhomogeneous samples, data analysis requires a more flexible set of tools.

In order to analyze data from multiple beamlines in a robust fashion, we have developed a $M A T L A B$-based toolbox for data analysis. The toolbox consists of three separate modules to separate data reading from data processing and visualization, as sketched in Fig. 3.7. The overall structure was chosen to have data-related tasks separated syntactically; however, key functionality is also accessible via standalone methods that are not bound to a certain module. All methods are designed to make simple tasks easy and complex tasks possible. The toolbox is available on GitHub (https://irpgoe.github.io/nanodiffraction/). A detailed documentation can be found in the internal documentation.

Finally, we believe that the described toolbox can contribute well to the analysis of arbitrary scanning diffraction data, alongside more complex data analysis suites, such as $D A W N 2$ [141], DPDAK [142], SMC [143] or $M M W-I$ [144], that most often base their workflow on a graphical user interface. Hence, the described toolbox should be understood as a means to simplify data analysis of arbitrary data. A clean syntax should make it easy to understand and use offline or online, during an experiment, even for users with little background in computer programming. More experienced users should also easily be able to add new data analysis methods.

\section{A2. Example dataset and visualization}

An example dataset obtained at the ID13 nanohutch endstation on a similar sample has been made available online at Zenodo [145], that can be used to test the functionality of the data analysis toolbox. The example data set and a representative PCA analysis of the data is shown as an example in Fig. 3.8. Example code (including calibrated experimental parameters) for these data is provided in the 


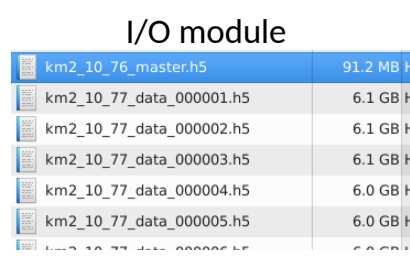

file location parameters file naming parameters

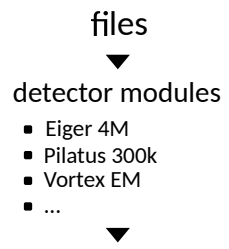

file reading

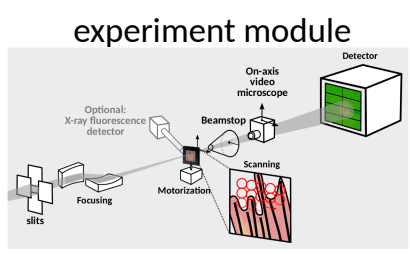

E scan parameters geometrical parameters

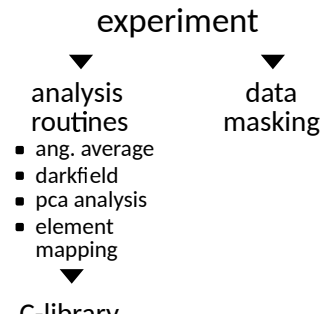

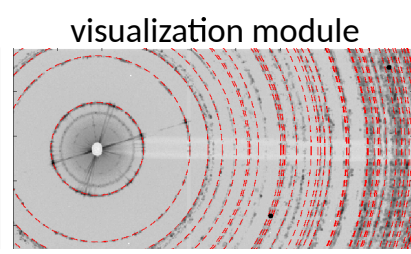

visualization settings

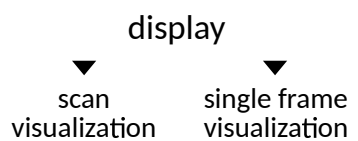

Figure 3.7.: Illustration of the modular design approach and workflow of the proposed data analysis toolbox for scanning diffraction experiments. Data treatment is separated into three main tasks: data reading, analysis and visualization. The I/O module 'files' is initialized with parameters related to the file storage and naming conventions used, and file reading routines are contained for each detector in a seperate module. Data can be requested by the experiment module that contains parameters of the experiment and can perform standard analysis procedures in a batch processing manner. Finally, parameter maps or single frames can be easily visualized by the 'display' module.
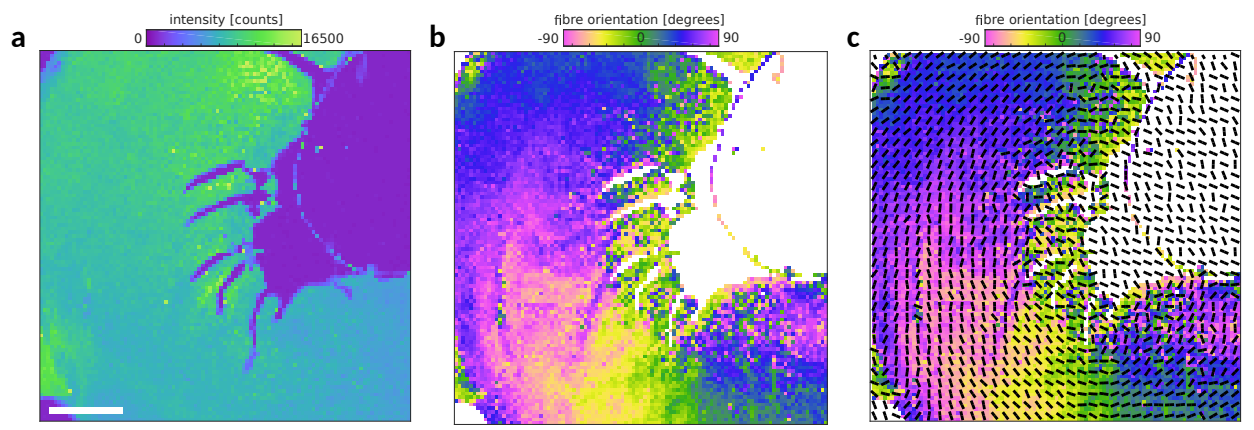

Figure 3.8.: Example dataset. Integrated scattered intensity and fiber orientation shown in (a) and (b), respectively. Note that, besides color scaling, a quiver plot (c) might be a reasonable choice to visualize orientations. Scale bar: $400 \mu \mathrm{m}$.

data analysis toolbox. 


\section{Acknowledgements}

We thank Tilman Grünewald for excellent support during beamtime. The work has been carried out within the framework of the Partnership for Soft Condensed Matter (PSCM) of the ESRF with continuous support by Diego Pontoni.

\section{Funding information}

Funding for this research was provided by: Deutsche Forschungsgemeinschaft through Sonderforschungsbereich 937/project A11. 



\section{X-ray diffraction imaging of cardiac cells and tissue}

J.-D. Nicolas, M. Bernhardt, S. F. Schlick, M. Tiburcy, W.-H. Zimmermann, A. Khan, A. Markus, F. Alves, K. Toischer and T. Salditt

Reproduced from Progress in Biophysics and Molecular Biology, in press (2018).

With the development of advanced focusing optics for X-rays, we can now use Xray beams with spot sizes in the micro- or nanometer range to scan cells and large areas of tissues and continuously record the diffraction signals. From this data, Xray scattering maps or so-called X-ray darkfield images are computed showing how different types of cells or regions of tissues differ in their diffraction intensity. At the same time a diffraction pattern is available for each scan point which encodes the local nanostructure, averaged over many contributing constituents illuminated by the beam. In this work we have exploited these new capabilities of scanning Xray diffraction to investigate cardiac muscle cells as well as cardiac tissue. We give examples of how cardiac cells, especially living, cultured cells, can be prepared to be compatible with the instrumentation constraints of nano- or micro-diffraction instruments. Furthermore, we show how the developmental stage, ranging from neonatal to adult cells, as well as the final preparation state of the cardiomyocytes influences the recorded scattering signal and how these diffraction signals compare to the structure of a fully developed cardiac muscle.

\subsection{Introduction}

Our current understanding of force generation in skeletal and heart muscle is to a large extent based on the structural analysis of the highly ordered acto-myosin assembly in the sarcomere as well as the corresponding structural dynamics, for example under different mechanical loading. Classical X-ray diffraction studies of skeletal [74] and heart muscle [128] have helped to elucidate the basic structure of the sarcomere. In contrast to electron microscopy, an important advantage of 
X-ray diffraction for the study of muscle structure is the fact that diffraction is compatible with in situ recordings under mechanical load, under different physiological parameters, and in parallel to measurements of the contractile force. With the advent of brilliant third generation synchrotron radiation, molecular details of the myosin head dynamics, binding, and stroke have been unraveled in seminal diffraction experiments $[14,25,26,75]$.

In these experiments, however, structural information is averaged over macroscopically large volumes of the muscle tissue, without any real-space resolution. This classic approach maximizes the signal-to-noise level, but impedes the observation of local structures. Without sufficient real-space resolution, the correspondance between the sarcomeric structure and the cardiac cytoarchitecture is obscured. Yet, we know that the cytoarchitecture shows characteristic variations of fibril orientation, density and histological appearance. One can speculate that these functionally relevant structures on micron scales are coupled to corresponding changes at molecular scale. In order to understand the mechanism of force generation beyond average values, mapping of structural variations is required, with real-space resolution down to sub-cellular scales.

Continued progress in X-ray optics has enabled diffraction experiments with spot sizes in the micron and even nanometer range [113], well-suited to record the smallangle X-ray scattering (SAXS) of complex soft-matter structures [146], biomaterials $[28,139]$, or biological cells with spot sizes smaller than a single organelle [41-44, $62,147]$. Recently, we have aimed at resolving the cytoskeletal structure of single cardiomyocyte cells at different preparation states. In particular, we have aimed at recording diffraction signals of the state when cells have started to actively contract and striation patterns are observed in fluorescence microscopy images. In corresponding experiments $[44,148]$ we have been able to observe sufficiently strong anisotropic diffraction patterns, for example in the case of single cardiomyocytes isolated from neonatal rats. In these experiments the probing X-ray beam was raster scanned over the sample and a scattering pattern was recorded at each scan point. The overall concept of scanning X-ray diffraction in the small-angle regime as applied to cardiac cells and tissue is summarized in Fig. 4.1. The signal could also be linked to the underlying actin cortex, for example by correlation to fluorescence microscopy using fluorescently labeled actin. At the same time, a clear diffraction signature of a sarcomeric complex was not observed. While the diffraction pattern from a micron or sub-micron spot is certainly expected to differ in strength, functional form, and resolvable range of momentum transfer (q-range), the absence of any typical muscle diffraction peak was rather obscure. Certainly, 
the level at which the acto-myosin is expressed in neonatal cells is much smaller than in adult cells [149]. The fixation and preparation of cells possibly also play a significant role. Finally, problems of radiation damage are expected to be accentuated when concentrating X-ray photons to a small spot size.

All of these issues called for a broader and deeper investigation of cell type, preparation pathway, sample environment, and X-ray optical parameters, which is now provided in the here presented work. While the results provided here are original, the present manuscript also serves as a mini-survey and review of all challenges which we have encountered over the last three years in the application of scanning micro- and nano-beam diffraction to heart muscle cells. We present results obtained for three different types of cells: (i) cardiomyocytes from neonatal rats, (ii) human induced pluripotent stem cells (iPS) differentiated to cardiac muscle cells, and (iii) cardiomyocytes from adult mice. As a benchmark, and for comparison, we also present scanning X-ray diffraction results obtained on heart tissue slices from mouse. This comparison shows that much stronger signal levels can be achieved for tissues than for isolated cells. We also show diffraction signals from engineered heart muscle (EHM), which represents early postnatal human myocardium [150]. For this overview, we have included data obtained for different preparation and fixation states, from simple freeze-dried to cryogenic vitrification and the alive state, and address the experimental challenge in each case. Finally, we include experimental results on different optical settings from micro- to sub-micro-, and nanometer spot sizes, as well as different synchrotron experiments and optical setups.

Most importantly, we can now demonstrate that for the case of adult cardiomyocytes, diffraction originating from acto-myosin complexes can indeed be observed with micro-beam focusing optics. In this way, sub-cellular resolution is now enabled by micro-focus diffraction studies of muscle cells. We expect that extension of the approach presented can shed light on how structural parameters vary within cells and tissues on functionally relevant scales.

The manuscript is organized as follows. Following this introduction, we describe micro- and nano-beam experiments on cells in different preparation states in Sec. 4.2, covering freeze-dried, cryogenically protected as well as living cell preparations. Then, we outline briefly how the data recorded on cardiac tissue can be used to generate parameter maps that show structural parameters that are specific to the acto-myosin complex. The analysis is extended to studies on adult cardiomyocytes in Sec. 4.4 and we show how such a study can profit from the correlation with fluorescence microscopy in Sec. 4.5. The article then closes with a summary 
and outlook.

\subsection{Different sample preparation states and X-ray optical setups}

\subsubsection{Freeze-dried cells}

Initial attemps to target single, isolated cells using focused $\mathrm{x}$-radiation used dried cell preparations to prevent radiation damage, or, more precisely, to limit the diffusion of free radicals generated by X-rays. Dried preparations offer secondly the advantage that the prepared samples can be very easily handled and mounted into a standard beamline setup. We have followed this approach and performed initial scanning diffraction studies on freeze-dried cardiomyocytes.

The workflow to freeze-dry cells cultured on a silicon nitride window is described elsewhere $[43,130]$ and can be summarized as follows: First, cells were cultured on a coated, $1 \mu \mathrm{m}$ thick silicon nitride window (Silson Ltd.) with a frame size of typically $5 \mathrm{~mm}$. Then, the cells were chemically fixed in $9 \%$ formaldehyde solution (Merck KGaA). Typically, after chemical fixation we permeabilized the cell membrane (0.5\% Triton X-100, SigmaAldrich Co. LLC), and specifically labeled f-actin (Phalloidin Alexa 488, Invitrogen) and DNA (Hoechst 33342, Invitrogen) to visualize the actin network as well as the cell nuclei. Right before the plunging process, the window with the cells was washed with triethylammonium acetate salt-free buffer (TEAA, 85.6 mM in $\mathrm{H}_{2} \mathrm{O}$, Merck KGaA). The sample was then blotted using standard blotting paper and plunged into a $-195^{\circ}$ cold ethane/propane bath for vitrification using a Leica Gridplunger (Leica EM GP, Leica). Finally, the cells were transferred into a cryo vial under liquid nitrogen and transported into a custom-built lyophilisator. Two synchrotron experiments were dedicated to the structure analysis of freeze-dried cells. The experiments took place at the GINIX endstation of the P10 beamline at PETRA III, DESY and the nanofocus endstation of beamline ID13, ESRF. The experimental parameters of the beamline configurations are summarized in Tab.4.1.

Figure 4.2 gives an overview on results that can be extracted from the scattering data recorded on freeze-dried cell preparations. Data shown in Fig. 4.2(A-D) was recorded at $\mathrm{P} 10$ while the remaining data was obtained at beamline ID13. In the preparations described here, the scattering data can be correlated to the fluorescence micrographs recorded prior to freeze-drying. Additional fluorescence micro- 

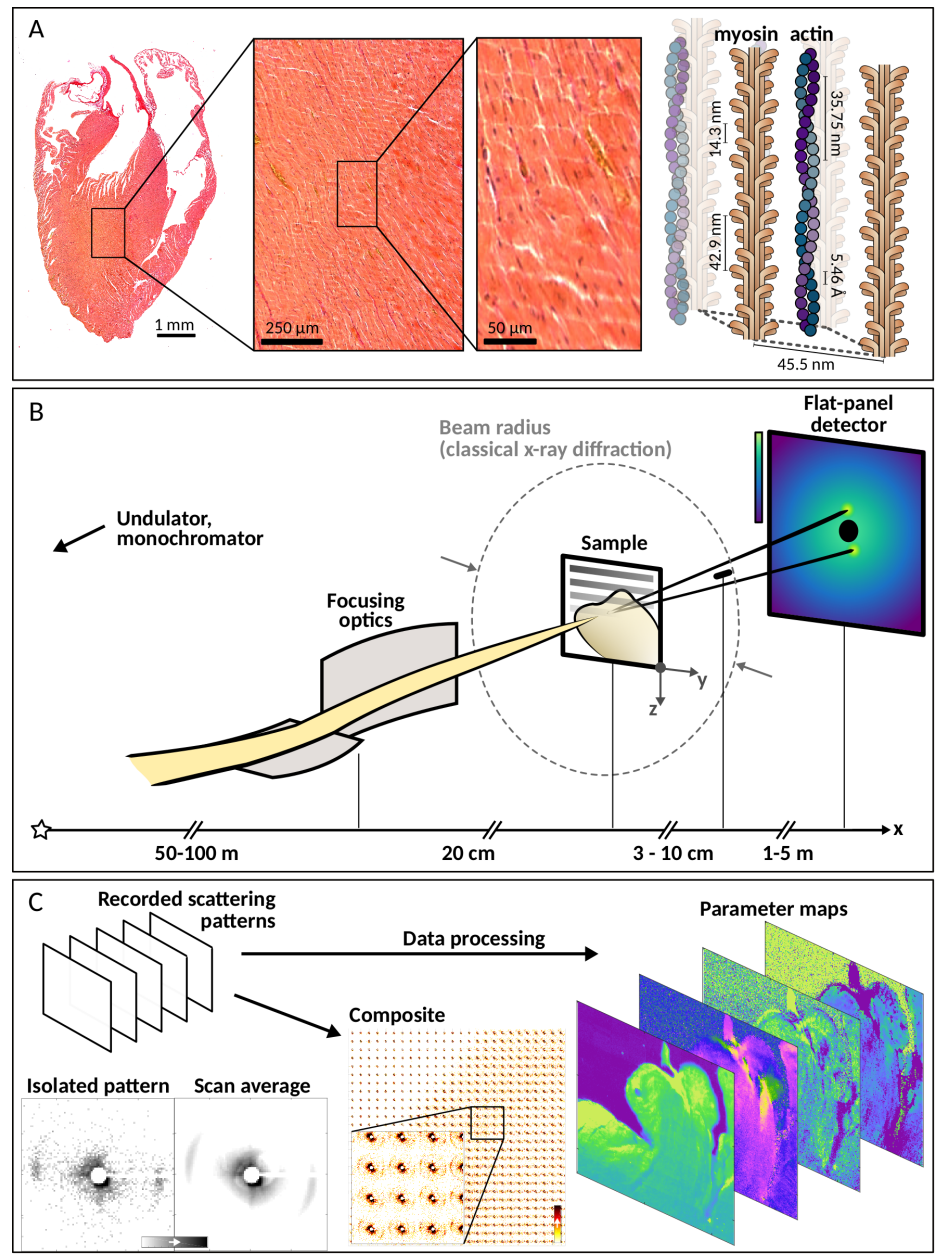

Figure 4.1.: Experimental concept. (A) Murine cardiac tissue on multiple length scales. (left) A picrosirius red-stained longitudinal heart section together with (center) two different magnifications, and scheme of myosin and actin fibers in an hexagonal arrangement (right). Fundamental helical repeat distances are indicated as well as the unit cell and the lattice constant. (B) Simplified overview of the experimental setup for scanning diffraction studies. $X$-rays can be focused by e.g. focusing mirrors, the sample is motorized with piezoelectric motors and a single photon counting detector is employed and synchronized to the motor stage for data recording. Further downstream, a small absorber blocks the primary beam to protect the detector and to reduce air scattering. Finer sample features can be mapped with this approach compared to classical diffraction experiments which employ much larger beam diameters. (C) Recorded scattering patterns obtained during a scan can either be reduced to a single scattering pattern or displayed with respect to their scan position. Each scattering pattern can also be analyzed in view of structural features (parameter maps). 
graphs were recorded after the freeze-drying process (data not shown) to verify that the cross-striation visible in the fluorescence signal is preserved, indicating that myofibril structure remained intact. Fig. 4.2(A) depicts a fluorescence micrograph of multiple cardiac muscle cells with cross-striation. Note, that in this example we have used neonatal rat cardiomyocytes obtained after isolation from young rat as previously used e.g. in [148]. The cells were isolated following the procedure described in $[151,152]$. For neonatal rat cardiomyocytes the window was pre-coated with a solution containing fibronectin and gelatine $(0.5 \%$ fibronectin and $0.08 \%$ gelatine in sterile $\mathrm{H}_{2} \mathrm{O}$ ). In the corresponding darkfield map in Fig. 4.2(B), nuclei are well contrasted while the myofibril orientation in Fig. 4.2(C) is extracted from the scattering and can be depicted by color using the cyclic color map design from [131]. In this representation, the color is representative for the myofibril orientation in the range of $\left[-\frac{\pi}{2}, \frac{\pi}{2}\right)$. The corresponding direction of the scattering is rotated by 90 degrees, into the range $[0, \pi)$.

Next, we averaged all scattering patterns from the scan and integrated the resulting image azimuthally. The intensity in this representation is plotted as a function of the wave vector transfer $q_{r}$, defined as $q_{r}=\frac{4 \pi}{\lambda} \sin (\theta)$ where $\lambda$ is the wavelength of the radiation and $2 \theta$ is the scattering angle. The index $r$ is used to clarify that we refer to the radial component of the wavevector. The azimuthally integrated curve, also referred to as the one-dimensional structure factor, is shown in Fig. 4.2(D). At large $q_{r}$ the structure factor decays according to the power law $I\left(q_{r}\right) \propto q_{r}^{-c}+d$ where c denotes the Porod exponent and $d$ the noise level of the experiment. Here, we obtained a Porod exponent of $c=3.9$ from a power-law fit. The importance of the Porod exponent is derived from its property to classify the statistical properties of the underlying structure from which photons are scattered in a very concise and condensed manner. The Porod exponent finds wide spread use in the characterization of structure of macromolecular solutions, suspensions and gels investigated by small-angle X-ray scattering experiments. While it is model independent as such, different models of geometric structure can easily be tested against the measured exponent. Since more recently, the Porod exponent is also used to characterize the more complex cytoskeletal network of cells, see e.g. [41-44].

The anisotropy of the scattering can inform on how oriented the scattering structure is on average in a given point and in which directions scattering is strongest. For cardiomyocytes the principal directions of the scattering patterns are typically correlated with the local orientation of the actin filaments [44]. More generally, it reflects the direction and strength of macromolecular polarisation, which is an important descriptor of the local cell structure. Note that the scattering signal is a 
sum over many different contributors, not just actin alone. The degree to which the scattering is anisotropic is quantified by the unitless anisotropy parameter $\omega$ [44]. Following a principal component analysis (PCA) of the scattering distribution, one can first assign two eigenvectors to each scattering pattern, where the larger eigenvector $\vec{v}_{1}$ is pointing along the primary scattering direction while the smaller eigenvector $\vec{v}_{2}$ is orthogonal to $\vec{v}_{1}$. The filament orientation shown in Fig. 4.2(C) corresponds to $\vec{v}_{2}$, based on the fact that scattering from a cylindrical structure extends furthest perpendicular to the main axis. From the corresponding eigenvalues $\lambda_{1}$ and $\lambda_{2}$, which have a meaning in themselves related to the predominant length scale of scatterers respectively, one can compute the unitless anisotropy parameter,

$$
\omega=\frac{\lambda_{1}-\lambda_{2}}{\left|\lambda_{1}+\lambda_{2}\right|} .
$$

This value is confined to the interval $[0,1]$ where 0 corresponds to a complete isotropic signal and 1 to a perfectly anisotropic, oriented scattering distribution.

In Fig. 4.2(E-G) we give an example, where the characterization of the anisotropy can be useful. In this example we have used induced pluripotent stem cells (iPS) that were reprogrammed from cells of human gingiva into their pluripotent state and redifferentiated into cardiomyocytes. For a detailed description of this procedure, see e.g. [150]. For optimal adherence of iPS cells to the silicon nitride membrane, it was coated using Synthemax $(0.025 \mathrm{mg} / \mathrm{ml}$; Corning Synthemax II-SC substrate, Merck KGaA) coating solution. Furthermore, iPS cells were chemically fixed in $4 \%$ formaldehyde solution (Roti-Histofix 4\%, Roth $\mathrm{GmbH}+$ Co. KG). The darkfield map is shown in Fig. 4.2(E). From the orientation map in (F) one can read that the actin filaments run horizontally and the myofibrils can be most specifically visualized in the anisotropy map depicted in $(G)$. Along the myofibrils shown in $(\mathrm{G})$, the anisotropy even appears periodically modulated as one could expect from the cross-striated nature of the fibrils.

One should add that the PCA approach is in certain cases not suitable for the extraction of the orientation and anisotropy of the scattering. Similar results can however be achieved by calculating the circular mean and variance of the onedimensional intensity profile $I(\phi)$ that has been obtained by radial integration over a certain $q_{r}$-range. We suggest to resort to the circular mean and variance if the overall signal is very low and other scattering contributions with a preferred directionality are superimposed onto the signal of interest. An example will be given further below. Note, that the circular variance is defined within $[0,1]$ as in the case of the anisotropy, only, that a value of 1 now corresponds to a 
perfectly isotropic signal. Further, note that all analysis routines have been implemented in the nanodiffraction toolbox, described in [46] and available from GitHub (https://irpgoe.github.io/nanodiffraction/).

Table 4.1.: Experimental configuration of beamline P10/GINIX, DESY and beamline ID13, ESRF during scanning diffraction experiments on freeze-dried and living iPS cells.

\begin{tabular}{lll}
\hline Beamline & P10 (DESY) & ID13 (ESRF) \\
\hline Energy (keV) & 13.8 & 14.64 \\
Monochromator & Si $(111)$ double cryst. & Si $(111)$ channel-cut mono. \\
Detector & Pilatus 300k (Dectris) & Eiger 4M (Dectris) \\
Detector distance $(\mathrm{m})$ & 5.12 & 1.93 \\
Focus $\left(\mathrm{nm}^{2}\right)$ & $350 \times 430$ & $150 \times 150$ \\
Focusing & Kirkpatrick-Baez mirrors & Compound refractive lenses \\
$\mathrm{I}_{0}(\mathrm{ph} / \mathrm{s})$ & $8.86 \times 10^{11}$ & $9.64 \times 10^{10}$ \\
Beamstop & $17 \times 12 \times 0.28 \mathrm{~mm}^{3} \mathrm{Si}$ foil & $300 \mu \mathrm{m}$ diameter, $\mathrm{Pb}$ wire \\
& $8 \times 7 \times 0.025 \mathrm{~mm}^{3} \mathrm{~W}$ foil & \\
\hline
\end{tabular}

\subsubsection{Micropatterning}

So far, order parameters have been deduced but no specific acto-myosin diffraction signal was observed. We have hypothesized that in contrast to fully developed muscle cells, the cytoskeleton of neonatal cells simply does not express the same degree of ordering due to missing mechanosensing cues. Multiple cues can lead to a preferential alignment and ordering of the cells, such as micropattering, shear flow or mechanical and electrical stimulation. We have chosen to use micro-patterning as a technique to stimulate the development of aligned myofibers. The sample preparation technique was adapted from protocols in [153] and [154]. All experimental parameters of the X-ray experiment are listed in Tab.4.1.

First, a master mold was fabricated. For this purpose, a negative photoresist was spin coated onto a silicon wafer and developed according to a custom design. A $100 \mathrm{~nm}$ thin layer of chromium was then deposited and the photo-resist was removed in a lift-off process. The actual grooves could now be etched into the wafer by using reactive ion etching (RIE). The final design of the master mold features $20 \mu \mathrm{m}$ thin lanes. The depth of the grooves was approximately $10 \mu \mathrm{m}$.

To cast a stamp, a polymer (Sylgard 184 silicone elastomer kit, Dow Corning) is mixed with a curing agent (Sylgard 184 silicone elastomer kit, Dow Corning) and poured over the Si master. The stamp is then degassed in a vacuum cabinet for $10 \mathrm{~min}$ and baked afterwards for $2 \mathrm{~h}$ at $65^{\circ} \mathrm{C}$. Then, the PDMS is peeled off the master wafer and cut to an appropriate size for further usage. The stamp 

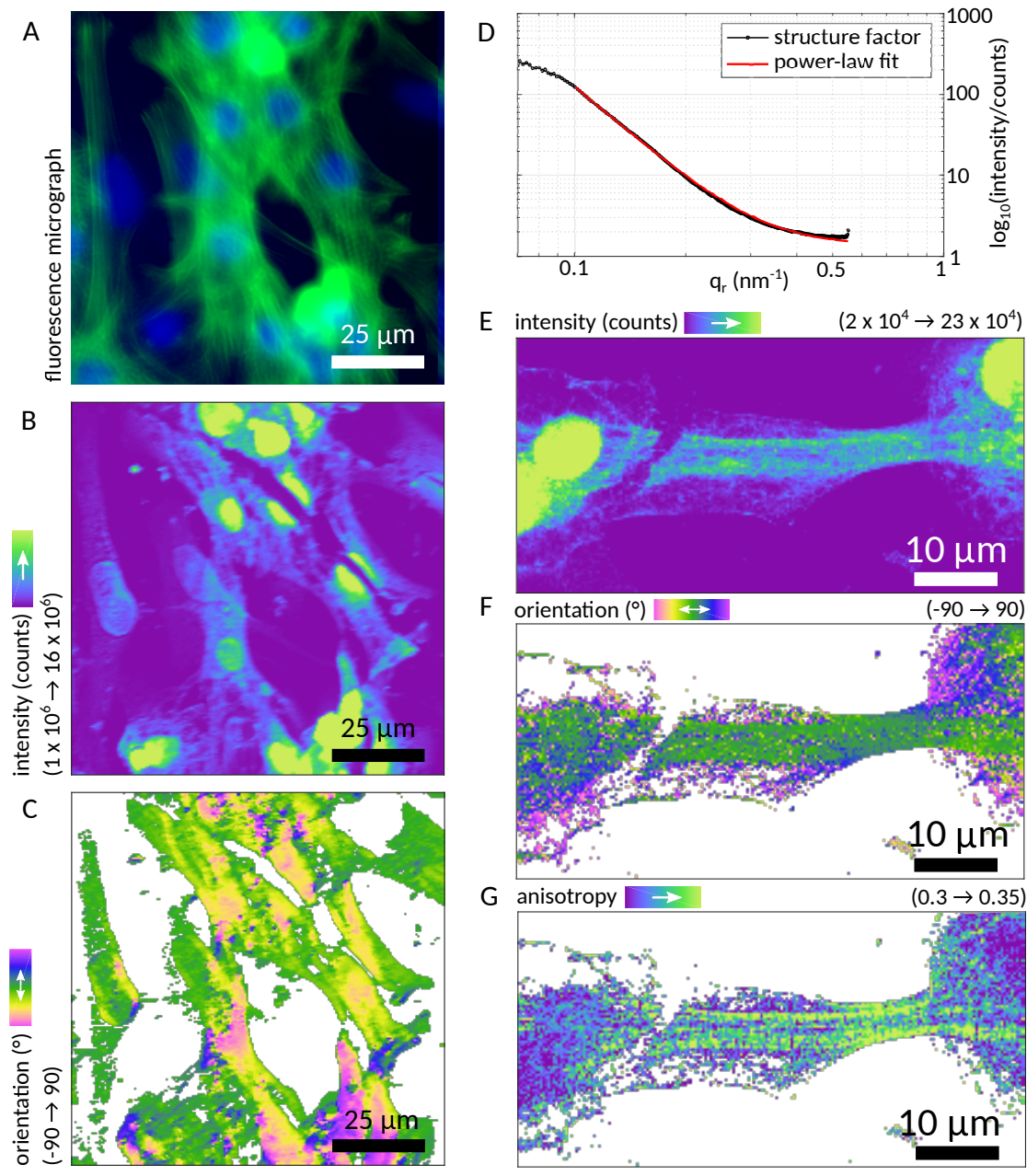

Figure 4.2.: Fluorescence micrographs, X-ray darkfield and orientation maps on single, freeze-dried cardiomyocytes. (A) Optical image and (B) X-ray darkfield map of neonatal rat cardiomyocytes. (C) Map of the local filament orientation. Scan points with a scattered intensity below $1 \times 10^{6}$ counts were colored white. Note that in regions of very low anisotropy, the orientation is biased towards zero degrees (green). The onedimensional structure factor obtained after azimuthal integration of the average of all scattering patterns from the scan is shown in (D). The power-law decay can be fitted and a Porod exponent assigned to the structure. (E) X-ray darkfield map of an iPS derived cardiomyocyte. Once again, the orientation in $(F)$ is obtained by PCA analysis. The anisotropy contrasts more specifically the highly oriented actomyosin filaments. $(G)$ Along the filament axis, the anisotropy appeared modulated. This modulation originated from the striated actomyosin filaments. Scan points in $(F-G)$ with a scattered intensity below $2 \times 10^{4}$ counts were colored white. 
is turned upside down and $10 \mu \mathrm{l}$ to $50 \mu \mathrm{l}$ fibronectin coating solution is pipetted onto the grooved stamp surface. After $30 \mathrm{~min}$, the stamp is washed three times in phosphate-buffered saline (PBS) and let dry. The stamp can now by carefully placed onto a clean silicon nitride window that was made hydrophilic by means of plasma cleaning for $5 \mathrm{~min}$ in a plasma cleaner (Expanded Plasma Cleaner PDC002, Harrick Inc.). A small weight of $30 \mathrm{mg}$ is added to enhance the transfer of the coating layer onto the silicon nitride. The weight has to be well adjusted to prevent the thin window from breaking. The stamp casting and coating process is summarized in Fig. 4.3(A).

A fluorescence micrograph proving a successful transfer of the coating layer and culture of iPS derived cardiomyocytes on the coated lines is shown in Fig. 4.3(B). We have applied this approach and imaged iPS derived cardiomyocytes cultured on a micropatterned surface. An example is shown in Fig.4.3(C). The fluorescence micrograph shows that the myofibrils were indeed intact as indicated by the cross-striations of the myofibrils in the fluorescence signal. Even though the micropatterning approach did not lead to a significant increase of the small angle scattering signal, we could observe that the anisotropy map in Fig. 4.3(E) correlates very well with the fluorescence micrograph in $(\mathrm{D})$, underlining once more that it can represent the degree of alignment of the filaments. From (E) one can even infer that one myofibril is most prominently aligned, indicated by an arrow, while the same statement cannot be made for the fluorescence signal in (D).

\subsubsection{Cryogenic recordings}

Following experiments on freeze-dried preparations, it was necessary to test whether this invasive sample preparation procedure had caused the absence of a clear actomyosin diffraction signature. To test this hypothesis, we have performed scanning diffraction experiments on (i) cryo-protected as well as (ii) vitrified cells. While (i) is simply a means to prevent rapid decay of macromolecular structure after irradiation as in cryo-crystallography, (ii) in addition avoids dehydration artefacts and structural changes already before the X-ray recording. Note that for (ii), the thin water layer surrounding the cells is quasi-instantaneously turned to an amorphous state by rapid plunge freezing (vitrification), and cells are covered by this vitreous layer at all times. The native structure is thus preserved, unless flaws in the handling and the sample transfer result in ice formation when the temperature rises above the glass point. The fact that the rapid cooling rate indeed 'fixes' macromolecular structures is very well established from electron microscopy. For both 


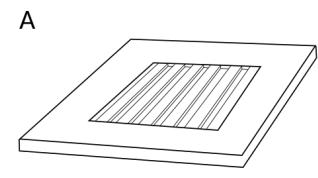

Si master

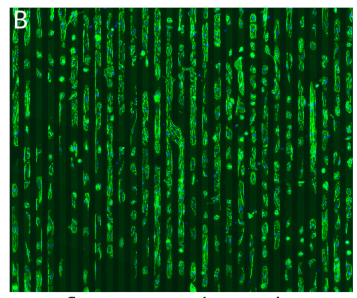

fluorescence micrograph

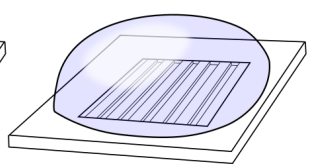

PDMS casting

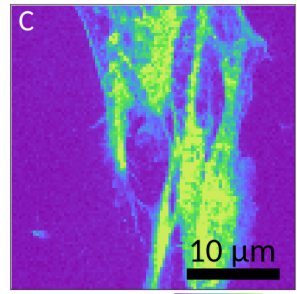

intensity (counts) $\left(1 \times 10^{4} \rightarrow 12 \times 10^{4}\right)$

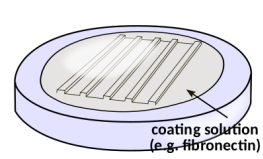

PDMS stamp

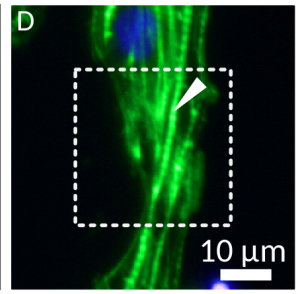

fluorescence micrograph

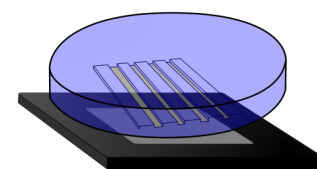

stamping on $\mathrm{SiN}$

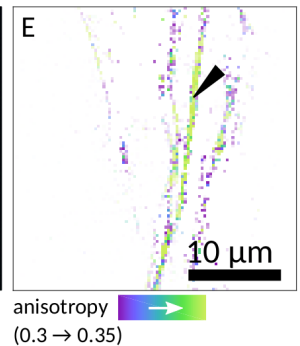

Figure 4.3.: Microcontact printing. (A) Preparation of PDMS stamps for micropatterning. Grooves of $20 \mu \mathrm{m}$ width and $10 \mu \mathrm{m}$ height were etched into the silicon wafer using reactive ion etching. PDMS was cast over the silicon master. The PDMS stamp was cut into the desired size and shape and the flat side is coated with fibronectin coating solution. Finally, the stamp could be used for transferring the fibronectin coating onto the silicon nitride window. A weight was usually placed onto the PDMS stamp to transfer the coating evenly onto the substrate. Fluorescently labeled actin filaments of iPS derived cardiomyocytes cultured onto micropatterned substrates are shown in (B). Note that cardiomyocytes attached only to the stamped fibronectin lines. (C) Darkfield map of iPS derived cardiomyocytes on a micropatterned window. Striation of the fibrils was observed in the micrograph in $(D)$. The most strongly aligned myofiber, indicated by an arrow in $(D)$ and $(E)$, can be found by referring to the anisotropy map in (E). Here, background was removed from the graphic by additionally scaling the color from white to fully saturated within the range 0.3 (white) to 0.35 (saturated).

cases, (i) and (ii), the cryogenic temperature during the X-ray recording increases the window of tolerable X-ray dose, and hence also the possibility to detect weak signals. For (i), the sample should ideally be free of water. Here, we have used freeze-dried cell preparations for this purpose, and have inserted them into a cold nitrogen gas stream $(T \approx 170 \mathrm{~K})$. Vitrified samples have to be kept at cryogenic temperatures at all times, either by mounting the sample onto a cold finger and inserting the sample into a vacuum chamber, or by rapid mounting of the sample into a cold nitrogen gas stream. Cryogenic chambers for X-ray experiments on vitrified samples have been described before [155-157] but are highly specialized developments with limited access and flexibility, even though the demand for such developments is currently rising [95, 158, 159].

In the following, we describe experiments using an $\mathrm{N}_{2}$ Cryostream (Oxford Cryosystems). The general workflow was described earlier [43] and can be summarized as 
follows: First, cells are cultured on a $1 \mu \mathrm{m}$ thick silicon nitride window with a frame size of typically $5 \mathrm{~mm}$. In this case, the frame size is chosen such that it fits the cryogenic stream of approximately $7 \mathrm{~mm}$ diameter. The samples are then vitrified as in the case of freeze-dried preparations and stored in cryovials under liquid nitrogen until right before the experiment.

The preparation at the beamline consists of four steps, see Fig. 4.4(A-E). First, a cryovial is opened and the window is placed into the groove of a metal block to facilitate mounting the sample into the magnetic sample holder. The window is then gently moved into the opening slit of the sample mount. Once inserted, the sample is fixed by a screw. The magnetic sample mount is then once again covered by a plastic vial that is attached magnetically to the mount. So far, all steps were conducted under liquid nitrogen. The sample is now prepared to be taken to the experiment, where the cryostream is put in place and operating. Placing the sample into the cryostream without exposing it to room temperature is crucial and the bottleneck in the sample processing queue. We typically proceeded by taking the vial with the sample out of the cryobath and placing it onto the magnetic mount of the sample tower. At this point, the vial should still contain liquid nitrogen. Then, the sample typically needed to be quickly rotated such that the sample surface is parallel to the stream direction and the cover is removed as fast as possible to avoid potential heating. The final arrangement is shown in Fig. 4.4(F).

Fig. 4.4(G) shows the X-ray darkfield map of cryoprotected iPS derived cardiomyocytes which are bridging two fibronectin-coated lines. The corresponding fluorescence image recorded prior to freeze-drying is shown in Fig. 4.4(H). The background in $(\mathrm{G})$ has a grainy structure stemming from ice crystals due to water adsorption at the cold sample surface. After approximately one hour of experiment time, we have observed ice crystal formation (I) at the center of the silicon nitride window. During the course of this experiment, ice crystal formation was very difficult to prevent, possibly due to the fact that the cold $\mathrm{N}_{2}$ stream was not perfectly shielded by a coating of dry $\mathrm{N}_{2}$ atmosphere. Fig. 4.4(K) shows a sample of vitrified iPS derived cardiomyocytes on a homogeneously coated silicon nitride surface. In this particular case, we could observe the vitrified water layer in the beamline's on-axis optical microscope. Again, the iPS derived cardiomyocytes can be recognized in the X-ray darkfield. In this case, the background exhibits a 'network-like' pattern. Based on the diffraction signal, it became apparent that intensity in the background was caused by ice cracks which exhibited strongly anisotropic and modulated diffraction signals. The vitrified iPS derived cardiomyocytes give rise to predominantly isotropic scattering, and with no sign of any 
particular ordered acto-myosin structure such as in muscle.

The experiments also illustrate well which technical challenges are still associated with scanning X-ray diffraction of cells. This is even more the case for studies of living cells, as reported in the next section.

Table 4.2.: Experimental configuration of beamline P10/GINIX, DESY during scanning diffraction experiments on cryoprotected and vitrified cells.

\begin{tabular}{ll}
\hline Beamline & P10 (DESY) \\
\hline Energy $(\mathrm{keV})$ & 13.8 \\
Monochromator & $\mathrm{Si}(111)$ double cryst. \\
Detector & Eiger $4 \mathrm{M}$ \\
Detector distance $(\mathrm{m})$ & 5.12 \\
Focus $\left(\mathrm{nm}^{2}\right)$ & $309 \times 301$ \\
Focusing & Kirkpatrick-Baez mirrors \\
$\mathrm{I}_{0}(\mathrm{ph} / \mathrm{s})$ & $7.75 \times 10^{10}$ \\
Beamstop $\left(\mathrm{mm}^{2}\right)$ & $10 \times 7,0.2 \mathrm{~mm}$ thick $\mathrm{Cu}$ foil \\
\hline
\end{tabular}

\subsubsection{Living cells}

For living cells, the strategy must be opposite to cryogenic recordings. Instead of 'freezing' the structure and rendering it more 'radiation resistant', one must now try to outrun radiation damage in time by very quick shots, each on pristine sample regions. Dose rate becomes the key quantity, not just dose, and scan points must hence be spaced sufficiently wide. The extreme case of such a 'diffract before destroy' principle has turned into reality by the development of free-electron laser (FEL) radiation sources, which are uniquely suited to target the living state of biological matter [160-163]. From a preparation point of view, living samples do not require processing and are hence easier to prepare. However, the accurate design of sophisticated X-ray compatible sample chambers requires great care. In this respect, three basic concepts are generally followed: (i) static chambers that typically consist of two windows glued together and containing a small, microlitersized amount of liquid (see e.g. [164]), (ii) microfluidic chambers [165, 166], and (iii) jet or droplet injection systems [70].

Here, we describe the use of a static chamber for scanning diffraction experiments with the possibility to extend the chamber design to microfluidic applications. The chamber set was purchased from Silson (Silson Ltd.). It consists of two opposing silicon nitride windows. A polymer spacer is deposited on one window. Since the height of the SU8 spacer material can be very accurately controlled in the man- 

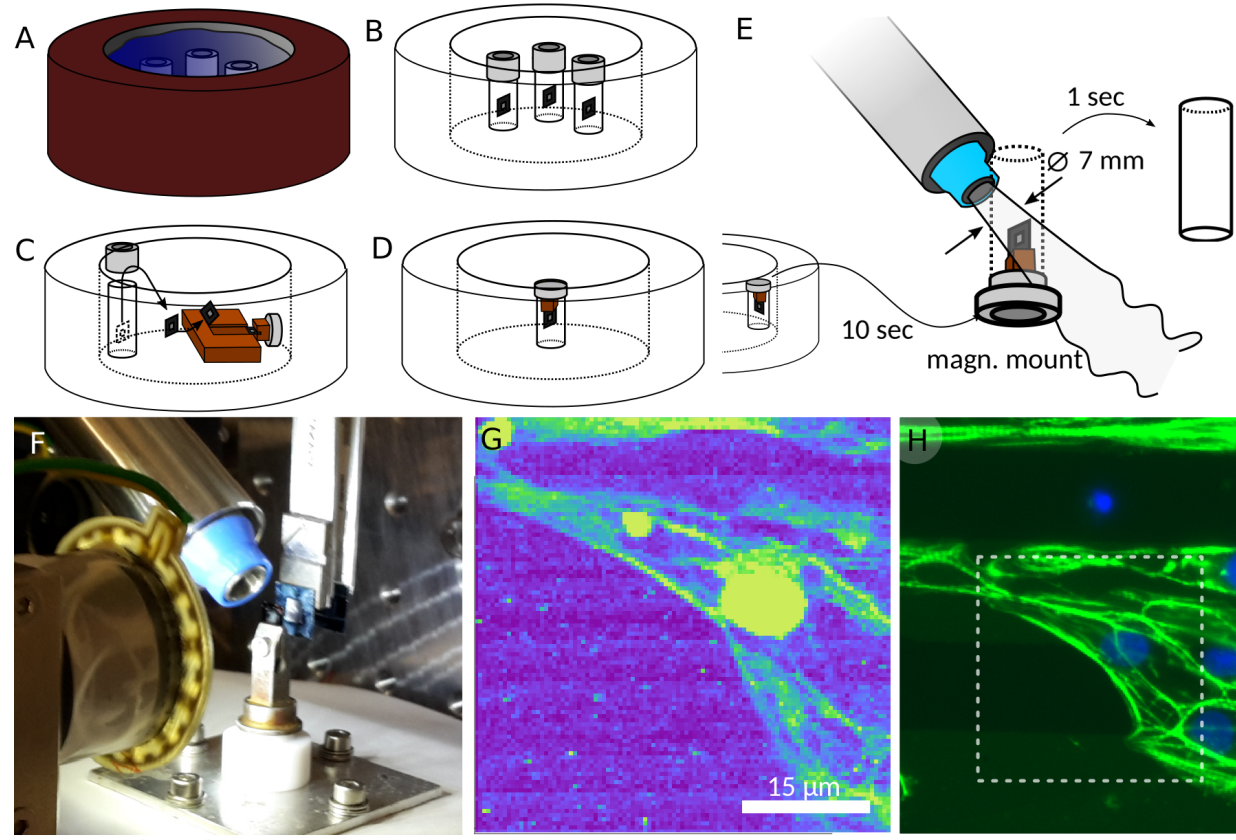

beamline setup
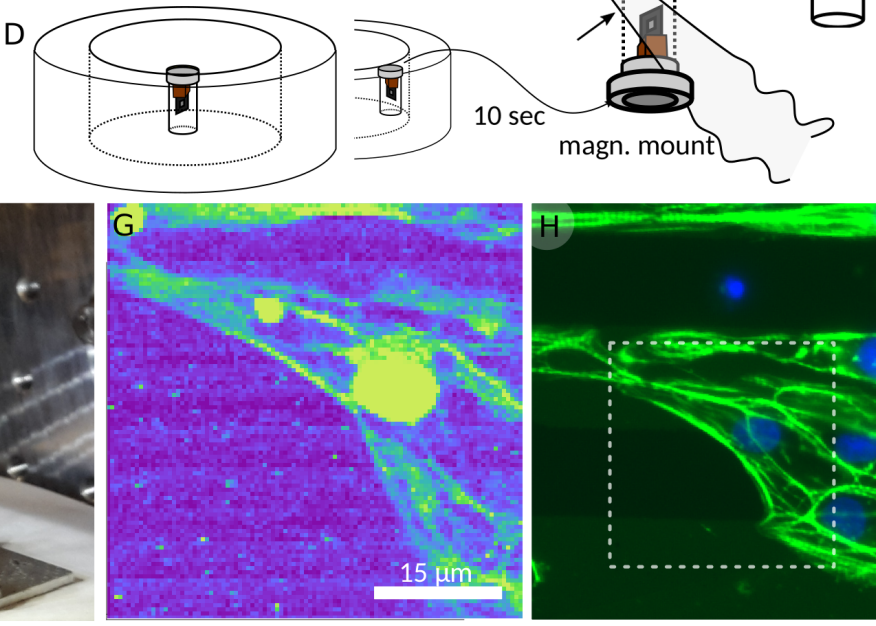

intensity [counts]

(min: $\left.2.6 \times 10^{4}, \max : 3.2 \times 10^{4}\right)$

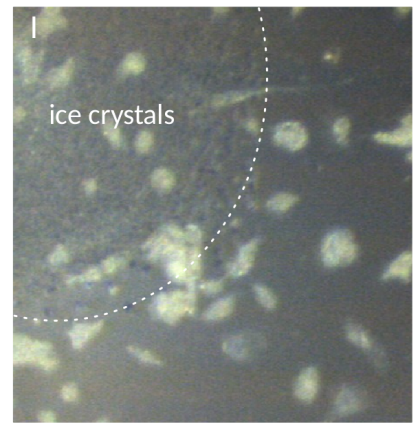

ice crystal formation

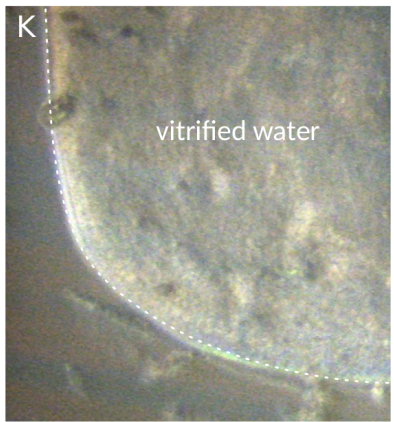

vitrified water layer

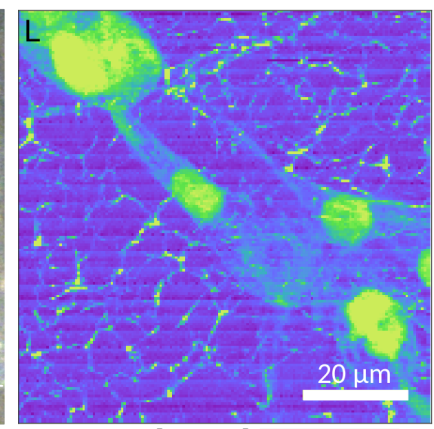

intensity [counts] $\overrightarrow{\left(\min : 19 \times 10^{4}, \max : 23 \times 10^{4}\right)}$

Figure 4.4.: Cryopreservation in scanning X-ray diffraction experiments. (A-F) Mounting of the sample into the beamline setup. (A) Dewar with vials, (B) 'X-ray vision' sketch, $(C)$ mounting of the sample into the sample holder under liquid nitrogen, $(D)$ prepared sample on magnetic mount, (E) mounting the sample onto the motorized stage into the cryostream. The cap has to be removed quickly in order to prevent thawing of the sample. (F) Photograph of the mounted sample. $(G)$ Darkfield contrast map of a freeze-dried cardiomyocyte, cryoprotected using a cryostream. (H) Fluorescence micrograph with a white dashed box indicating the area corresponding to the scanned region in $(G)$. After several minutes, ice crystals started to form on the silicon nitride membrane, as can be seen in (I). A mounted, vitrified sample is shown in $(K)$ and the corresponding darkfield map shown in $(L)$. 
ufacturing process, the overall height of the water layer can be adapted to the sample under consideration. Here, we have chosen a spacer thickness of $70 \mu \mathrm{m}$ and $100 \mu \mathrm{m}$ to accomodate the cells and leave a remaining sufficiently large volume so that products of metabolism do not accumulate too rapidly. The cells, in this case iPS-derived cardiomyocytes, are cultured for 3 to 7 days on the window without the polymer spacer. The chamber is then closed by carefully placing the window containing the polymer spacer onto its counterpart. To tightly seal the chamber, it is inserted between two aluminium frames that are carefully screwed together. The assembled chamber can then be inserted into a sample holding pin. The overall design of the chamber is shown in Fig. 4.5(A). This design was tested during two consecutive beamtimes at beamline P10 (GINIX endstation), DESY and ID13, ESRF.

The experimental parameters for both experiments are summarized in Tab. 4.1 (ID13) and Tab. 4.2 (P10). In both experiments, we have used iPS derived cardiomyocytes. Fig. 4.5(b) shows a darkfield map of a dense population of iPS derived cardiomyocytes in their liquid environment. The radiotoxic effect on the cells was most easily verified by later visual inspection of the cells in an optical stereo microscope available at the nearby safety level 2 laboratory of the Biomedical Facility at the ESRF, as shown in Fig. 4.5(c). Two scanned regions are clearly visible due to the apparent lack of cells. Interestingly, we found in this example that the adjacent cells were still actively contracting, hereby confirming that the chamber design is indeed well suited for X-ray experiments.

The average of all scattering patterns is shown in Fig. 4.5(D). The isotropic scattering can be summarized in the one-dimensional structure factor. The azimuthally integrated structure factor of a single scattering pattern, is shown in Fig. 4.5(E). The power-law fit resulted in a value of $c=3.0$ for the Porod exponent.

In the experiment at the Göttingen nanofocus setup (GINIX endstation) of beamline P10, cells were not as densely populated as in the previous case. Isolated cells can be very well seen in the darkfield image in Fig. 4.5(F), however, from their shape one can infer that the cells are already in an apoptotic or necrotic state. Re-inspecting the cell chamber under the optical microscope confirmed this assumption. It is not surprising that radiation damage progressed faster than the scan area could be covered. For this reason, we recorded scattering patterns at isolated locations that were not yet exposed to X-rays (data not shown). Such patterns were again predominantly isotropic, which gave additional evidence that myofibrils in iPS-derived cardiomyocytes are not as well ordered on the nanoscale as one might expect based on the striation pattern observed in fluorescence 


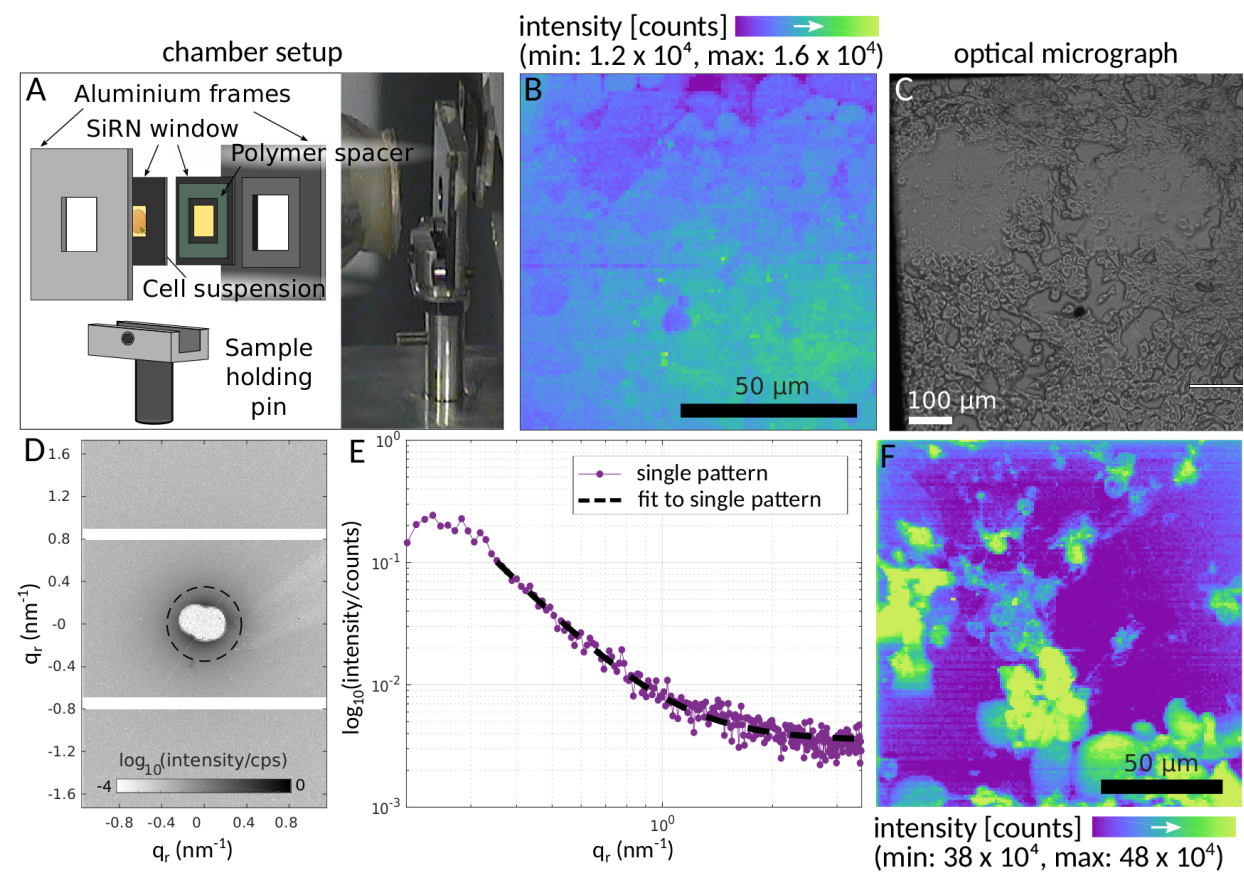

Figure 4.5.: Scanning X-ray diffraction on living iPS derived cardiomyocytes. (A) Sketch and optical image of the assembled static chamber for experiments on living cell cultures. Two silicon nitride windows were sandwiched between two aluminium holders and tightly sealed to avoid leakage. (B) Darkfield map of a dense population of cells in the liquid chamber recorded at beamline ID13, ESRF. (C) Optical micrograph recorded with a stereomicroscope after the $X$-ray experiment was performed. Damage due to the intense $X$-ray beam led to cell death in the scanned areas. Cells from non-exposed areas were still contracting. (D) Average scattering pattern obtained from the scan in (B). A Porod fit to the structure factor decay of an isolated pattern is shown in (E) with a Porod exponent of 3.0. (F) Darkfield map of a less dense population of cells obtained from an experiment at beamline P10/GINIX, DESY.

micrographs, and the ability of the cells to contract.

\subsection{Cardiac tissue}

All cell preparations presented thus far made use of cardiomyocytes either from neonatal rats or human iPS cells as indicated, which allowed for culturing of the cells on silicon nitride windows. We showed here that multiple structural parameters can be extracted from the small-angle scattering signal. However, modulations of the scattering, either originating from the filament arrangement in an hexagonal 
lattice or from helical repeats of the myosin or actin filaments, was lacking. We hypothesized that the invasive sample preparation in the case of freeze-dried cells, even when aligned by micropatterning, might be the leading cause for the absence of layer lines, i.e. interference from the helical assembly of the filament subunits, or interfilament interference. However, due to the fact that the interfilament interference was not present in the case of living and vitrified cells, we conclude that due to the immaturity of the cardiomyocytes cultured in 2D sarcomeric structure is simply not yet fully developed. We therefore conducted a scanning diffraction study on cardiac tissue to verify that in histological sections of an healthy, adult mouse heart, modulations stemming from interfilament interference can be detected. The samples were mounted between two polypropylene foils as described in [46]. Key experimental parameters are summarized in Tab.4.3(left).

Indeed, Fig. 4.6(A) shows a scattering pattern and a map of multiple averaged scattering patterns obtained on heart muscle tissue obtained from adult mouse. The interfilament interference, i.e. the $(1,0)$ and $(1,1)$ reflections, can be clearly seen together with the directionality and anisotropy of the scattering signal. Note, that these reflections stem from the hexagonal array of filaments in the A-band of the sarcomere. The interplanar spacing $d^{(1,0)}$ is related to the reflection position $q_{r}^{(1,0)}$ by $d^{(1,0)}=\frac{2 \pi}{q_{r}^{(1,0)}}$. In turn, the $(1,1)$ reflection position is obtained by $q_{r}^{(1,1)}=$ $\sqrt{3} q_{r}^{(1,0)}$. Lastly, the lattice constant $a$ is identical to the spacing of the myosin filaments and is given by $a=2 d^{(1,0)} / \sqrt{3}$. In the following maps, we report the $(1,0)$ reflection position $q_{r}^{(1,0)}$. To generate e.g. the darkfield map shown in Fig. 4.6(B), we have used a 'stitching mode' to scan the tissue: Since the scanning range of the piezo motor used for continuous scanning of the sample could only cover an area of $200 \times 200 \mu^{2}$, multiple scans (patches) were performed at adjacent locations with exactly $0 \%$ overlap and consisting of $26 \times 26$ scan points per patch. The darkfield map is generated by displaying all patches next to each other in the appropriate ordering. The tiling grid is superimposed onto the darkfield map for clarity. Note, that Fig. 4.6(A) was also generated in this fashion, only, that in this case an average scattering pattern from each patch was calculated.

In a straight forward manner, we could now compute the orientation and anisotropy map as shown in Fig. 4.6(C-D), as well as a map of the $(1,0)$ reflection position. Note, that background is colored in white in Fig. 4.6(D-E), identified by a darkfield intensity below 700 counts. As we have reported in an earlier study on cardiac tissue [46] and contrary to our initial expectation, the reflection position is not constant throughout the tissue but varies continuously within $0.14 \mathrm{~nm}^{-1}$ to $0.17 \mathrm{~nm}^{-1}$ corresponding to a lattice spacing $d^{(1,0)}$ ranging from $37.0 \mathrm{~nm}$ to $44.9 \mathrm{~nm}$. In the 
literature lattice spacings between $35.0 \mathrm{~nm}$ and $38.3 \mathrm{~nm}$ are reported for intact, relaxed rat myocardium $[17,26]$. To this point a comparison is obscured by the fact that cells and tissue in the preparations used here were chemically fixed and the sarcomere length could not be simultaneously recorded which would be necessary as the lattice spacing is found to vary strongly with sarcomere length [17]. Once again, we found that the reflection position $q_{r}^{(1,0)}$ decreases close to the left ventricle of the heart. It was in this case obtained by azimuthally integrating every scattering pattern and fitting a model function of the form

$$
I\left(q_{r}\right)=a q_{r}^{-b}+c \exp \left(-\left(q_{r}-d\right)^{2} / \sigma^{2}\right)+e \exp \left(-\left(q_{r}-f\right)^{2} / \sigma^{2}\right)+g,
$$

to the structure factor. In this model, $a-g$ are fit parameters. Here, both reflections are modeled as Gaussians with positions $d$ and $f$. The reflection width was fixed at $\sigma=0.034 \mathrm{~nm}^{-1}$ during the fitting process.

Based on such maps, it is now possible to correlate such information to other modalities. This will be of particular importance if one aims at combining the information from scanning X-ray scattering recordings of the type presented in Fig. 4.6 with histology and immunohistochemistry in a bio-medical setting.

A similar analysis can now be performed on engineered heart muscle (EHM). The experimental parameters for this experiment are given in Tab. 4.3(right). The protocol for the preparation of EHM is described in [150]. Here, we have used circularly shaped EHM. The tissue was chemically fixed after 12 culture days in $4 \%$ formaldehyde solution and embedded in an agarose block for sectioning in a vibratome (VT1000 S, Leica Biosystems). Sections were cut to a thickness of $30 \mu \mathrm{m}$ and sandwiched between polypropylene foils with a small volume of excess PBS to keep the sample hydrated.

The darkfield map of EHM is shown in Fig. 4.6(F) together with the corresponding average scattering pattern shown in $(\mathrm{G})$. The darkfield map represents the tissue morphology which appears to be dominated by the collagen matrix, while modulations in the scattering patterns were absent. Since the muscle to matrix ratio in EHM is much smaller in comparison to bona fide heart muscle, the absence of sarcomeric reflections can be explained by the very low amount of myofibers in the scattering volume and a muscle diffraction signal is therefore simply not detected. Alternatively, since the cardiac tissue is composed of iPS-derived cardiomyocytes that have only been cultured for 12 days, the sarcomere might be far from exhibiting the long range order known from the 'adult' stage due to the immaturity of the cardiomyocytes. Further research and improved protocols for in tissue imaging are 

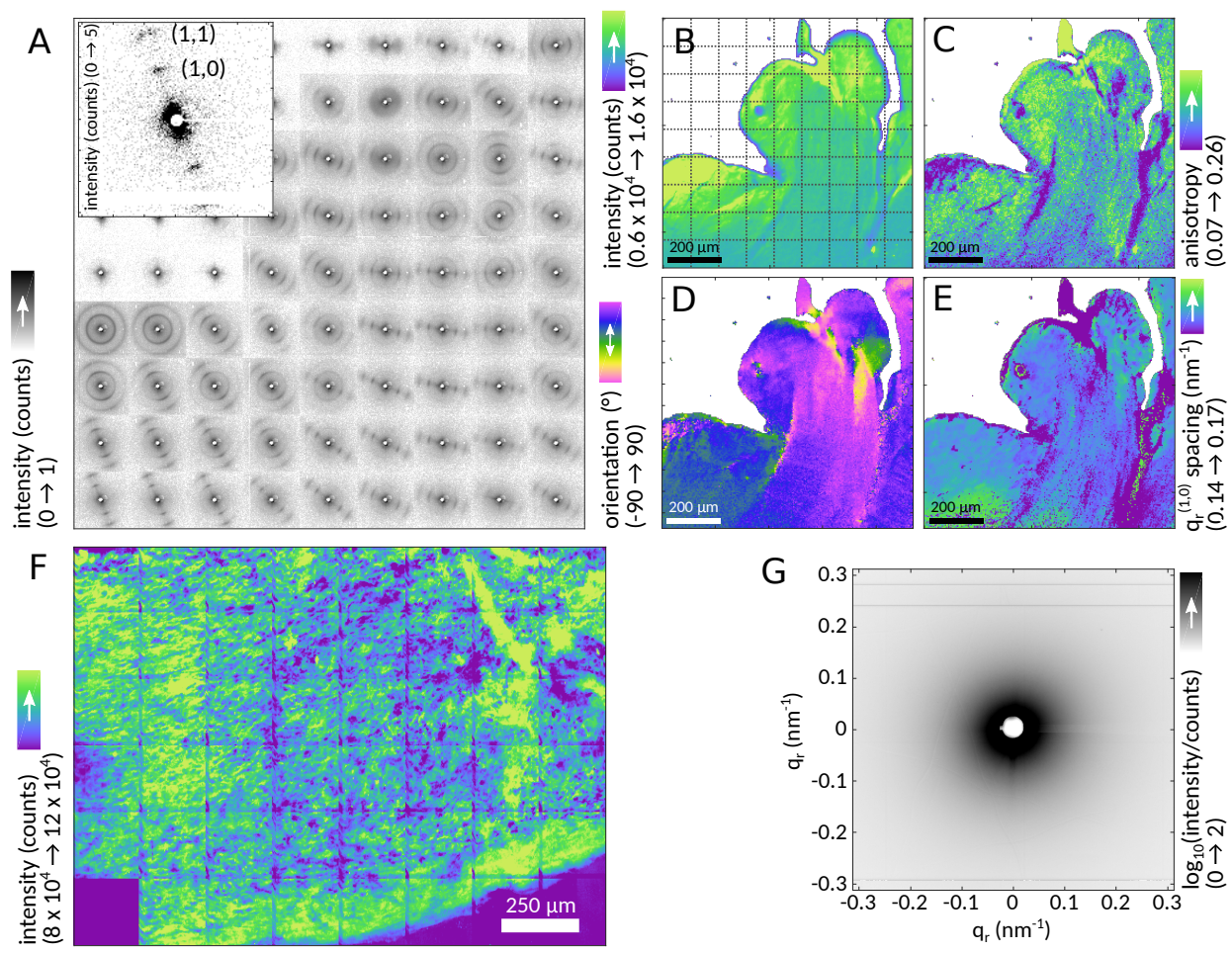

Figure 4.6.: Scanning X-ray diffraction on cardiac tissue. A section of heart muscle from an adult mouse was scanned and is shown in multiple contrast based on four different structural parameters extracted from the scattering distributions. (A) The entire image was recorded piecewise in 'stiching mode', such that small rectangular regions (fields) were scanned on a cartesian grid. Here, the fields were recorded with exactly $0 \%$ overlap. The average diffraction pattern of each field is shown in a composite image together with the scattering pattern from a single location. In (B) the darkfield map is shown and superimposed by the stitching grid for illustration. (C-E) Besides anisotropy and orientation of the scattering as in the case of the cell experiments, the $(1,0)$ reflections allowed for the extraction of the lattice constant of the hexagonal lattice of the actomyosin. In $(B-E)$ pixels with a darkfield intensity below 700 counts have been masked in white. $(F)$ Darkfield map of a $30 \mu \mathrm{m}$ thick engineered heart muscle (EHM), primarily depicting the collagen matrix. Scattering was dominantly isotropic and unlike the case of the adult cardiac tissue, reflections were not observed. The corresponding average scattering pattern is shown in $(G)$.

required to safely deduce how well, at the individual sarcomere level, the structure of EHM is developed in comparison to adult heart muscle. 
Table 4.3.: Experimental configuration of beamline P10/GINIX, DESY. Left: Parameters of the scanning diffraction experiments on adult mouse heart muscle as well as isolated, adult cardiomyocytes. Right: Parameters for the scans performed on engineered heart muscle.

\begin{tabular}{lll}
\hline Beamline & P10 (DESY) & P10 (DESY) \\
\hline Energy $(\mathrm{keV})$ & 13.8 & 13.8 \\
Monochromator & Si $(111)$ channel-cut mono. & Si(111) channel-cut mono. \\
Detector & Eiger 4M & Eiger 4M \\
Detector distance $(\mathrm{m})$ & 5.05 & 5.05 \\
Focus $\left(\mu \mathrm{m}^{2}\right)$ & $2.3 \times 2.3$ & $2.3 \times 2.3$ \\
Focusing & Compound refractive lenses & Compound refractive lenses \\
$\mathrm{I}_{0}(\mathrm{ph} / \mathrm{s})$ & $0.86 \times 10^{10}$ & $4.08 \times 10^{10}$ \\
Beamstop $\left(\mathrm{mm}^{2}\right)$ & $3 \mathrm{~mm}$ diameter, W wire & $3 \mathrm{~mm}$ diameter, W wire \\
& $0.1 \mathrm{~mm}$ diameter, W wire & $0.1 \mathrm{~mm}$ diameter, W wire \\
\hline
\end{tabular}

\subsection{Adult cardiac muscle cells}

Based on the fact that myofibers in adult tissue give rise to a specific acto-myosin signal that can be extracted from fits of model functions, it should in principle be possible to extract this signal from isolated single cells that were extracted from adult cardiac tissue. For experiments that were conducted at the GINIX endstation of beamline P10, we isolated cardiomyocytes from hearts of helthy adult mice as well as mice that developed heart fibrosis. As a model system for cardiac hypertrophy and fibrosis, we used TAC-operated (transverse aortic constriction) mice [167]. In this procedure, mice undergo a surgical procedure where the aorta is constricted by a small thread. TAC treated mice develop cardiac fibrosis after several weeks, which is characterized by an excessive deposition of collagen and other extracellular matrix proteins. We could hereby not only verify that the interfilament interference can be detected as in the case of tissue sections, as shown in Fig. 4.7(A), but also compare the internal structure of cardiomyocytes from healthy and diseased heart. Cells were isolated from the heart following the procedure described in [168].

For a thorough comparison, single cardiac cells were isolated from adult mice that have been (i) untreated, (ii) sham operated, and (iii) TAC-operated. In all cases, cells were chemically fixed immediately after the extraction from the tissue for conservation and to preserve the cellular structure until the experiment. For the experiments, once again a polypropylene chamber as in [46] was used: A small volume of cell suspension of around $30 \mu \mathrm{l}$ was pipetted onto a polypropylene window and a second window was placed on top. The chamber was sealed using nail polish to prevent drying of the cell suspension. To rule out any effect of the chem- 
ical fixative on the filament spacing and the expected scattering signal itself from the fixative used, we employed three different fixation solutions in all three heart preparations, namely $4 \%$ formaldehyde (FA) solution, $9 \%$ formaldehyde solution, and Karlsson-Schultz (K\&S) fixation solution [169, 170] containing 4\% formaldehyde and $2.5 \%$ glutaraldehyde. The absolute number of cells that were analyzed with respect to the original treatment of the heart and the chemical agent used for fixation is summarized in Tab.4.4.

In Fig. 4.7(A) we show one example of an optical micrograph of an adult cardiomyocyte, in this case extracted from a TAC heart and chemically fixed using the K\&S fixative solution. In contrast to cultured neonatal cardiomyocytes, adult cardiomyocytes have the commonly observed rod-shape. As expected, the scattering signal from such isolated cells was now similar to the scattering from adult cardiac tissue, even though the signal at a single location was much weaker. The corresponding average diffraction pattern (300 patterns, without background signal) and an isolated diffraction pattern shows the $(1,0)$ reflection in (B). This made it possible to perform a complete analysis of the diffraction signal as in the case of the tissue. In all cases, the scattered intensity, myofibril spacing, and the orientation could clearly be determined, as depicted in Fig.4.7(C),(D) and (E), respectively. In these maps, pixels with a darkfield intensity below 200 counts corresponding to background signal are masked in white. Due to the low signal levels, we have now resorted to the circular mean of the azimuthal intensity distribution as a measure for the filament orientation. Interestingly, the $(1,0)$ reflection position was varying significantly from cell to cell, while within a single cell it appeared rather constant throughout, see Fig. $4.7(\mathrm{~F})$ for a histogram of the $(1,0)$ reflection position obtained from the cell marked by an asterisk in Fig. 4.7(D).

To quantify the influence of the chemical fixation and mice treatment, in $(G)$ we show violin plots, i.e. normalized, symmetric vertical histograms, of the $(1,0)$ reflection positions of the myofibers respective treatment and fixation. From $(\mathrm{G})$ it becomes apparent, that even though the $(1,0)$ position within an isolated cell is rather constant, the reflection position from the population (cf. Tab.4.4), as we have seen previously in $(\mathrm{F})$ can vary more strongly. In $(\mathrm{G})$ the lattice spacing varies between $0.15 \mathrm{~nm}^{-1}$ and $0.20 \mathrm{~nm}^{-1}$ or in terms of $d^{(1,0)}$ between $31.4 \mathrm{~nm}$ and $41.9 \mathrm{~nm}$. It appears that the greatest difference is between the cardiomyocytes from TAC-treated mice that have been chemically fixed with K\&S and FA solution. This may reflect the pathological changes in this model which for example include calcium overload, which may lead to a variable hypercontractile state upon fixation. The results shown in $(\mathrm{G})$ are intriguing since the filament spacing and 


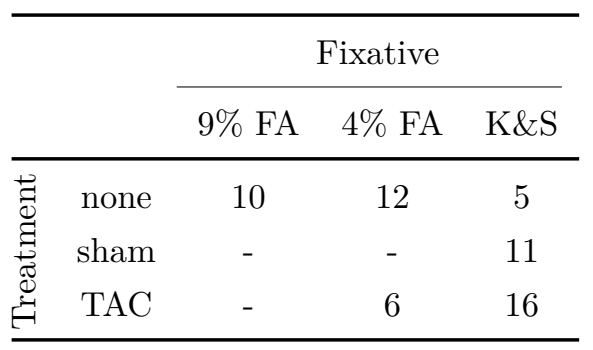

Table 4.4.: Number of cells analyzed based on preceding mouse treatment and chemical fixative used.

its distribution in an ensemble of cells might be potentially useful as a biomarker in comparative studies on cardiac diseases. At this point, however, the data obtained in this study does not yet conclusively show that the filament spacing is in fact an indicator for fibrosis. In addition, all fixation solutions employed here can in principle induce shrinkage. If one assumes isotropic shrinkage, then stronger crosslinking would induce smaller lattice spacings which is supported by the data shown in Fig. 4.7(G). However, a phosphate buffer was used in all fixation solutions, hence, relative changes inferred from Fig. 4.7(G) should still occur due to the cell model and not the fixation solution. Future experiments are required to clarify the influence of sample preparation on the filament spacing observed in such an experiment. Furthermore, the cells in this study were extracted from entire hearts which removes any local dependence. Since scanning diffraction maps of cardiac tissue shown here and in [46] have already shown that the filament spacing can vary locally within a similar range, one would have to use cells obtained from a specific location in the heart.

It is worth pointing out that the results presented in Sec. 4.3 and 4.4 where obtained using a micro-focused rather than a nano-focused beam. This relaxed focusing made it possible to image cardiac tissue and isolated cardiomyocytes at still moderate resolution while reducing the effective dose by approximately two orders of magnitude. This change was especially crucial for the case of adult cardiomyocytes where severe radiation damage by a nanofocused beam could potentially lead to structure degradation within a single exposure and would therefore inhibit the observation of interfilament reflections. 
A

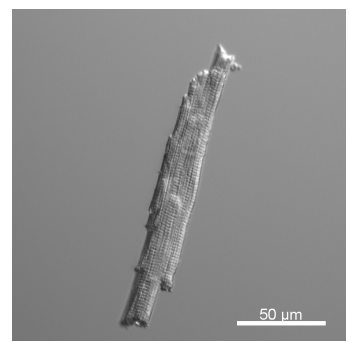

optical micrograph, brightfield

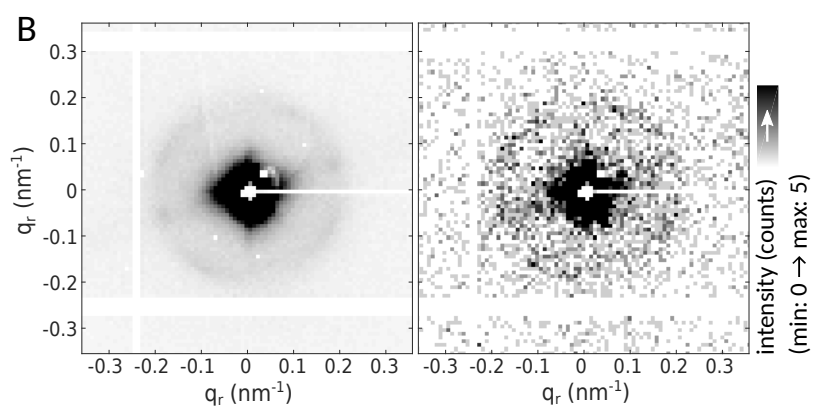

$\mathrm{q}_{\mathrm{r}}^{(1,0)}$ position $\left(\mathrm{nm}^{-1}\right) \quad \rightarrow \quad$ orientation $\left(^{\circ}\right) \rightarrow \leftrightarrow$

$(0.14 \rightarrow 0.25)$

$(-90 \rightarrow 90)$
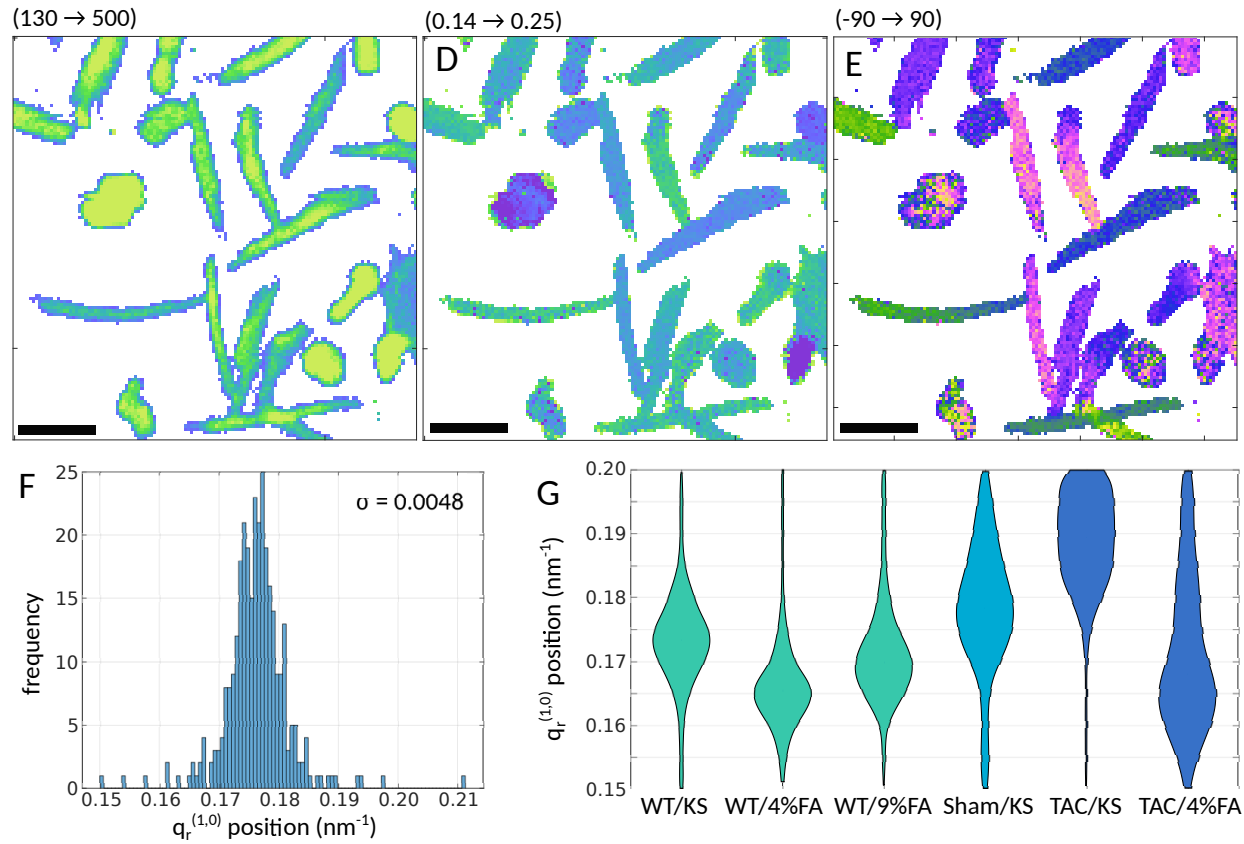

Figure 4.7.: X-ray structure analysis on isolated adult cardiomyocytes. (A) Optical micrograph of an adult cardiomyocyte. One example for an averaged and an isolated diffraction pattern from such a cell is shown in (B) and (C), respectively. (C) A darkfield map of isolated, hydrated, chemically fixed cells. (D) Position of the (1,0) reflection and (E) filament orientation could be determined pixelwise. Note, that in $(C-E)$, pixels with a darkfield intensity below 200 counts have been masked in white. Note, that the $(1,0)$ position in $(D)$ is not constant but can fluctuate from cell to cell around a mean $q_{r}^{(1,0)}$ by $\sigma=0.0048 \mathrm{~nm}^{-1}$ as shown in $(F)$ for the distribution of $q_{r}^{(1,0)}$ of the cell marked by in asterisk in $(D)$. (G) Different fixation solutions as well as different (patho)physiological conditions of the heart correspond to different filament periods. The distributions of the $(1,0)$ reflection positions of all cells from a certain preparation pathway are represented here as violin plots. Note, that the number of cells that were used for a given preparation pathway is summarized in Tab. 4.4. 


\subsection{Correlative microscopy}

Thus far we have exploited the $(1,1)$ and $(1,0)$ reflections as a structural observable that precisely reports on the order, disorder and packing of the filament lattice in the sarcomere. The real-space resolution in the scanning diffraction studies reported above was however limited by the moderate focusing required to avoid artefacts stemming from radiation damage. Incidentially, another means to specifically target the acto-myosin structure is by fluorescent labels. Using state-of-the-art fluorescence microscopy techniques such as confocal microscopy or super-resolution microscopy such as STED enables a very high spatial resolution down to the resolution range that can be accessed by means of scanning diffraction. In this respect, the limited real-space resolution in scanning diffraction can be extended by at least one order of magnitude when fluorescence micrographs of the same structure are recorded.

Such a combination of techniques can be particularly fruitful when the signal is originating from different sources, e.g. the fluorescently labeled cytoskeleton in the confocal or STED micrograph and the total scattered intensity reflecting Fourier components of the complete electron density of the sample. In this case, fluorescence microscopy can benefit from X-ray techniques which directly probe the electron density and thereby offer a highly quantitative contrast mechanism sensitive to the ensemble of biomolecular constituents.

Recently, we have introduced a novel approach of parallel STED or confocal microscopy recordings together with X-ray scanning diffraction and X-ray holography, all integrated into a single synchrotron endstation [73, 171]. Here we have made use of this setup to image adult cardiomyocytes. For instrumental simplicity and avoiding the technical challenges associated with chamber construction, we have used freeze-dried preparations as a first step. To this end, adult cardiomyocytes were deposited onto a silicon nitride window and fluorescently labeled using the STED-compatible dye Atto633 (AD 633-8X, Atto-tec), followed by freezedrying.

Fig. 4.8(A) shows a confocal stack of an isolated cardiomyocyte. In this case, we have not exploited the full STED-capability of the setup since recording an entire STED-stack of a cell would have greatly exceeded the available recording time. Fig. 4.8(B) shows a single confocal image from the stack, the average image and the maximum projection of the fluorescence intensity throughout the stack. The maximum projection hereby maps the maximum intensity that was recorded in a scan point in all frames for all scan points. The stack average appears rather 

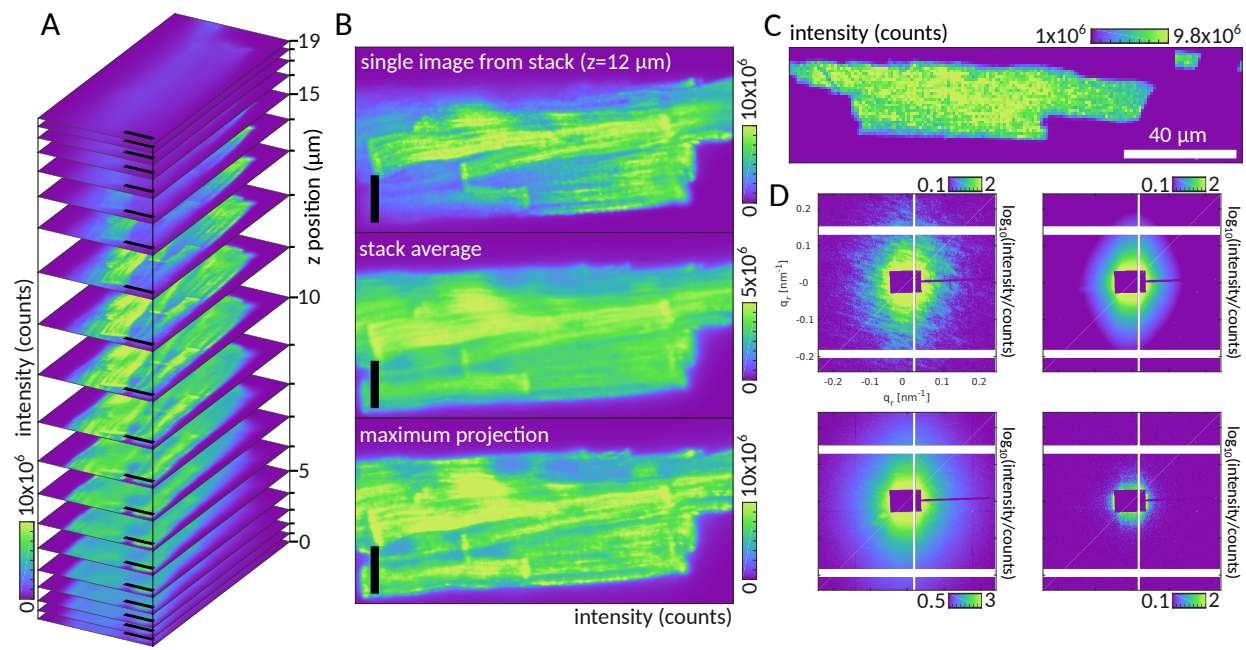

Figure 4.8.: Correlative microscopy. (A) Confocal stack through freeze-dried cardiomyocytes isolated from an adult mouse heart. The actin cortex was stained with Atto633. The image was recorded using a custom-built STED/confocal microscope that was setup next to the $X$-ray beam including a motorized sample translation stage to combine STED or confocal imaging with X-ray diffraction of X-ray holography [73, 171]. (B) Single image from the stack, stack average and a maximum projection through the entire stack. Since the X-ray darkfield map is sensitive to the projected electron density, we hypothesized that it will most closely resemble the stack average of the confocal stack. (C) Darkfield map of the cell shown in (B). All cellular components add to the signal and the filament structure is washed out. The presence of the myofibrils can be derived from from the anisotropy of the scattering. See $(D)$ for an isolated diffraction pattern $(D$, top-left), the average (D,top-right), and the maximum projection (D,bottom-left). For completeness, the background intensity is shown in ( $D$, bottom-right).

blurry, while the striated myofibrils are most clearly visible from the single image. Following the confocal recording, we have moved the sample into the focus of the Xray beam and scanned the sample through the focus position. For this experiment, the experimental parameters are given in Tab. 4.5. The darkfield image depicted in Fig. 4.8(C) is now a map of all cellular components that were traversed by the Xray beam. The anisotropic scattering due to the aligned myofibrils is apparent from the example scattering pattern shown in Fig. 4.8(D, top-left). For completeness, we have added the average intensity ( $\mathrm{D}$, top-right), maximum projection ( $\mathrm{D}$, bottomleft) and the background intensity (D, bottom-right). 
Table 4.5.: Experimental configuration of beamline P10/GINIX, DESY during confocal fluorescence microscopy and scanning diffraction experiments on freeze-dried cardiac muscle cells.

\begin{tabular}{ll}
\hline Beamline & P10 (DESY) \\
\hline Energy (keV) & 8.0 \\
Monochromator & $\mathrm{Si}(111)$ channel-cut mono. \\
Detector & Eiger 4M \\
Detector distance $(\mathrm{m})$ & 5.12 \\
Focus $\left(\mathrm{nm}^{2}\right)$ & $150 \times 150$ \\
Focusing & Kirkpatrick-Baez mirrors \\
$\mathrm{I}_{0}(\mathrm{ph} / \mathrm{s})$ & $8.86 \times 10^{11}$ \\
Beamstop $\left(\mathrm{mm}^{2}\right)$ & $17 \times 12,0.28 \mathrm{~mm}$ thick Si foil \\
& $8 \times 7,0.025 \mathrm{~mm}$ thick W foil \\
\hline
\end{tabular}

\subsection{Summary and synopsis}

In summary, we have developed, adapted and evaluated experimental tools for applying micro- and nanofocused hard X-rays to studies of cardiac cells and tissues. This includes beamline instrumentation, comparison of sample preparation and sample chambers, experimental controls, establishing dose limits, monitoring of radiation damage and automated data analysis. A main purpose of this study was to investigate how the X-ray scattering parameters and darkfield images differ in cardiomyocytes of different levels of maturity (lowest level in iPS-derived cardiomyocytes; highest level in cardiomyocytes from adult mice), and how this can be delineated from effects of sample preparation (cells vs. tissue) and experimental parameters (fixatives and cardiomyocytes from healthy and diseased heart). We have described as accurately as possible not only the approaches 'which worked best', but all configurations which were screened and tested. This is for two reasons. First, the work is still primarily method driven, and technical aspects, developments and parameters including the 'does', 'don'ts' should be clearly specified. Secondly, the absence of signal in many of the experiments are indeed the message. Precisely the fact that the highly structured diffraction pattern of classical muscle diffraction was lacking in monolayer cultures of immature cardiomyocytes and engineered heart muscle from iPS-derived cardiomyocytes, is the lesson learned.

In particular, we found that both for single cardiac cells as well as for tissue, cardiomyocytes from neonatal rats and derived from iPS cells do not give rise to interfilament interference or layer lines. The same was true for engineered tissue. The use of micropatterning for freeze-dried cells and the embedding of cardiomyocytes in a collagen matrix did lead to an alignment of cardiomyocytes in $2 \mathrm{~d}$ and 
$3 \mathrm{~d}$, respectively, but not to a more ordered internal structure on the level of the sarcomere. In all cases but the adult cardiac cells and tissues, reflections were not detected. This finding can be explained by a poor sarcomere organization, a loss of signal due to a too small number of myofibers in the scattering volume in the case of $2 \mathrm{~d}$ monolayers of cardiomyocytes, or a blurring of scattering images due to an overlay by highly abundant isotropically organized extracellular matrix.

More work has to be invested in ruling out all spurious effects and artefacts resulting from preparation, radiation damage, and background signal. Sources of artefacts are still many, owing from the still very early stage of the technique. Based on this, it will be of greatest interest to study the onset of what we would call the adult sarcomeric structure, along a maturation of the differentiation pathway. Providing a structural assay and a marker for the 'maturing structure', the presented approach could complement developments of cardiac research and in particular the translational research aming at the development of sarcomere-targeting therapeutics (e.g., acto-myosin modulators).

A second line of research to be exploited is to track down the surprisingly high inter-cell structural variability. Here we would like to understand how local structural changes relate to functions in cardiac tissue, and whether some of these changes could be exploited as structural biomarkers. In this respect, the compatibility of diffraction with screening a large number of cells, much in excess of what can be performed by electron microscopy, is encouraging. However, scaling of throughput will require more work on sample delivery techniques, and more specialized beamline endstations.

One must keep in mind that biological samples suffer from rather stringent radiation damage constraints. For this reason, we found it necessary in this study to sacrifice spatial resolution and use only moderate X-ray focusing to micron spot sizes. For future experiments, the selection of scan parameters to optimize the 'quality' of the diffraction signal, has to be drawn special attention to. We expect that with moderate focal spot sizes of around $10 \mu \mathrm{m}$ and lower background levels, achievable by reducing the propagation distance of the X-ray beam through air, higher orders of equatorial reflections as well as intense meridional reflections such as the M3 reflection will become detectable. In addition, the signal-to-noise ratio of the $(1,1)$ reflection will increase, and the ratio of the $(1,0)$ and $(1,1)$ reflection intensity could hence be exploited [137]. At the same time, experiments with nanofocused X-ray beams can still be useful, if radiation damage can be mitigated for example by cryogenic fixation. This also prevents the structural artefacts associated with freeze-drying. Scanning microscopy with nanofocused X-rays can help 
to localize and characterize acto-myosin complexes in non-adult cardiomyocytes attached to a substrate. In this case high lateral resolution in real space is more relevant than in thick adult cardiomyocytes where the structure is (a) more uniform and (b) anyway averaged over a large thickness along the optical axis. On these adult cardiomyocytes, however, alive recordings are possible since the signal level is high. Such measurements would best be performed with beamsizes on the order of the size of a single cardiomyocyte. Based on the homogeneous lattice spacing for a given cell, we expect that the overall structure per cell does not vary significantly and a larger beamsize would therefore not be a limitation, but would reduce radiation damage and help to cover a high number of dispersed cells in a chamber. In summary, micro-focus beams with spot sizes between $10 \mu \mathrm{m}$ and $100 \mu \mathrm{m}$ are recommended for structure analysis of tissue, isolated adult cardiomyocytes as well as living cells, while nano-focused X-ray beams can be useful for example to probe the acto-myosin cortex in less uniform cells such as non-adult cardiomyocytes or fibroblasts cells, provided that radiation damage can be mitigated.

In addition, correlative microscopy can help in guiding the diffraction experiment. More generally, combining X-ray imaging techniques with super-resolution fluorescence microscopy, provides additional contrast mechanisms which are to date poorly exploited. For X-rays, these contrast mechanisms are all associated with electron density, either its Fourier components (diffraction) or its projection images (coherent imaging, holography). This type of contrast is highly exploited in structural biology, but almost completely absent in cellular biology or biomedical research, except for - well - classical muscle diffraction, as addressed in the beginning of this manuscript.

\section{Acknowledgements}

We thank Kristin Müller for help in the preparation of iPS cells at the home institution and the biological laboratories of the synchrotron endstation P10 and the ESRF (Bio-medical Facility), as well as for implementing the micro-patterning technique on silicon nitride substrates. Mike Kanbach has helped in the fabrication of a silicon wafer used for micro-patterning. We are very grateful to Susanne Hengst for her dedicated help in cell culture and preparation, and Stefan Luther and Marion Kunze of Max Planck Institute for Dynamics and Self-Organization for providing the neonatal rat cardiomyocytes. We also thank Mohamed Belal for the preparation of TAC and sham mice and Alessya Jacqueline Kretzschmar for the extraction of the cardiomyocytes from the hearts. Manfred Burghammer and the 
team of the Bio-medical facility and the safety group of the ESRF provided an excellent work environment and support during beamtime at ID13. Michael Sprung is gratefully acknowledged for support during beamtimes at beamline P10. We also thank Erik Stellamanns for advice and support in using the biology laboratory and Markus Osterhoff for helpful discussions and the indispensable implementation of the continuous scanning modality at beamline P10 as well as useful tools for online data analysis. We acknowledge SFB937 Collective Behavior of Soft and Biological Matter / Project A11 and Project A18 for funding the research project on cardiac tissue cells, and BMBF/Verbundforschung (project 05K16MG2) for funding instrumentation at the GINIX endstation of the P10 beamline. 



\section{Imaging of neuronal tissues by X-ray diffraction and X-ray fluorescence microscopy: evaluation of contrast and biomarkers for neurodegenerative diseases}

E. Carboni*, J.-D. Nicolas*, M. Töpperwien, C. Stadelmann-Nessler, P. Lingor and T. Salditt

Reproduced from Biomedical Optics Express 8, 4331 - 4347 (2017).

* shared first authorship

We have used scanning X-ray diffraction (XRD) and X-ray fluorescence (XRF) with micro-focused synchrotron radiation to study histological sections from human substantia nigra (SN). Both XRF and XRD mappings visualize tissue properties, which are inaccessible by conventional microscopy and histology. We propose to use these advanced tools to characterize neuronal tissue in neurodegeneration, in particular in Parkinson's disease (PD). To this end, we take advantage of the recent experimental progress in X-ray focusing, detection, and use automated data analysis scripts to enable quantitative analysis of large fields of view. XRD signals are recorded and analyzed both in the regime of small-angle (SAXS) and wide-angle X-ray scattering (WAXS). The SAXS signal was analyzed in view of the local myelin structure, while WAXS was used to identify crystalline deposits. PD tissue scans exhibited increased amounts of crystallized cholesterol. The XRF analysis showed increased amounts of iron and decreased amounts of copper in the PD tissue compared to the control. 


\subsection{Introduction}

Parkinson's disease (PD) is the most common motor neurodegenerative disease worldwide and its prevalence is expected to dramatically increase in the near future because of the aging of the population [172]. The classical disease symptoms comprise (but are not limited to) motor symptoms such as rigidity, akinesia, rest tremor, and gait disturbance together with non-motor symptoms such as sleep disturbances, hyposmia and psychiatric symptoms. Despite intense research, PD diagnosis is still mostly relying on patients' symptoms and early biomarkers for the disease are still missing. In fact, only post-mortem analysis can ascertain PD diagnosis and exclude the presence of pathologies with similar symptomatology [173].

Histologically, PD brains show two well-known hallmarks: the depletion of dopaminergic neurons in the substantia nigra $(\mathrm{SN})$ and the presence of protein aggregates named Lewy Bodies (LB) that are mainly formed by a protein called alpha-synuclein (aSyn) [174]. In addition to that, also iron (Fe) is thought to play an important role in PD pathophysiology as Fe dyshomeostasis in the patients' brains is well documented [175]. Specifically, in PD there is an accumulation of Fe in the SN of the patients [176]. This is of great interest, since this particular brain region is one of the most affected by the disease. Similarly to other neurodegenerative diseases, PD patients display increased levels of oxidative stress and reactive oxygen species (ROS) [57]. Several cellular processes are responsible for ROS increase and, in PD, Fe accumulation is definitely one of the most prominent reasons. In fact, Fe and other metals can actively participate in the so-called Fenton and Haber-Weiss reactions leading to the formation of the highly reactive hydroxyl radicals thus exacerbating oxidative stress [177]. Together with Fe also dopamine is involved in the increase of oxidative stress. In PD, dopaminergic neurons are affected in the course of the disease leading to the appearance of motor symptoms. Dopamine metabolism is implicated in the generation of ROS [178]. Among dopamine byproducts, neuromelanin (NM) is noteworthy [179]. In contrast to other dopamine metabolites, NM is thought to exert a protective role against oxidative stress [180]. Histologically, NM is a dark-brown pigment inside dopaminergic neurons of SN (hence the name of this region). Therefore, due to its color, NM can be used in unstained sections of SN to localize the dopaminergic neurons. Oxidative stress leads to the production of unbalanced free radicals that are able to oxidize proteins, DNA and lipids in the cell. Among oxidized lipids in $\mathrm{PD}$, cholesterol and its oxidized byproducts (oxysterols) have shown to be involved 
in the exacerbation of the disease and might represent a possible biomarker for PD [181].

In this work, we explored whether scanning hard X-ray microscopy using focused synchrotron radiation can provide novel imaging modalities and 'structural biomarkers' in the context of PD research. To this end, we wanted to complement established histology of PD tissues by novel molecular contrast mechanisms and by two-dimensional (2D) mappings of these signals, yielding the micro-resolved spatial distribution of transition metals as well as the distribution of those molecular structures, which can be identified from their diffraction patterns. This is a timely effort, since significant progress in hard X-ray optics [27, 113] has now made it possible to rapidly scan tissue sections with micro- or nanometer focal spot sizes. By automated analysis, 2D maps of X-ray fluorescence (XRF) and diffraction signals can then be generated, providing advanced contrast mechanisms of scanning transmission X-ray microscopy (STXM). STXM with XRF contrast [182] has already become a fairly well established method for trace element mapping in neuronal cells and tissues, as reviewed in [53] and in [55]. In contrast to electron microscopy based X-ray microanalysis (EDX), XRF collects signal from the interior of the tissue section, with a sensitivity down to a few hundred atoms of an element in a pixel, and can also be used for speciation. In particular, the elemental distribution of $\mathrm{Fe}, \mathrm{Cu}, \mathrm{Zn}$, and $\mathrm{Mn}$, with suspected roles in redox regulation or signaling, have been addressed [54, 183-185]. In this context, the local spatial distribution is important. For example, it was shown by XRF mapping that neural cells redistribute significant pools of copper from their cell bodies to the periphery upon calcium activation [186]. Contrarily, scanning small-angle X-ray scattering (SAXS) and wide-angle X-ray scattering (WAXS) are largely unexplored for neural tissues, except for studies of myelin diffraction, see for example [47, 184]. These techniques have contributed to the understanding of biomaterials by local structure analysis $[28,122,139]$. This makes us confident that with further decrease of detection levels, scanning SAXS and WAXS can also be advantageously applied to soft biological tissues, in particular taking advantage of the recent experimental progress (e.g. focusing, detection, background suppression).

\subsection{Materials and methods}

Ethics statement: All samples were obtained from the UK Brain Bank (Parkinson's UK, London, England) and approved by the Multicenter Research Ethics Committee (07/MRE09/72). 
Demographics: PD sample (\#ID: PD102). Age: 81, Braak stage: 6, disease duration: 25 years, post-mortem interval: $16 \mathrm{~h}$. CTR sample (\#ID: MS523). Age: 63, Braak stage: N/A, disease duration: N/A, post-mortem interval: $21 \mathrm{~h}$.

Sample preparation: $30 \mu \mathrm{m}$ thick tissue sections from snap frozen tissue blocks from the midbrain of one PD patient and one non-PD control patient were cut in a cryostat (CM3050 S, Leica, Nussloch, Germany) using special teflon-coated blades (DB80 LX, Nussloch, Germany) to avoid iron particle contamination due to friction. During cutting, the sample temperature was kept at $-17^{\circ} \mathrm{C}$ given a sample holder temperature of $-15^{\circ} \mathrm{C}$. After cutting, the sections were deposited onto SiN membranes $\left(8 \times 8 \mathrm{~mm}^{2}\right.$ window size, $200 \mathrm{~nm}$ thickness, Silson Ltd., Southam, England) or onto $0.5 \mu \mathrm{m}-1 \mu \mathrm{m}$ thick, stretched polypropylene foils. The samples were then air dried for $15 \mathrm{~min}$ at room temperature (RT) and dried for $30 \mathrm{~min}$ at $60^{\circ} \mathrm{C}$. Finally, the samples were enclosed with a second $\mathrm{SiN}$ or polypropylene foil and sealed with nail polish.

X-ray fluorescence and scanning diffraction experiments: The samples were imaged at the ID13 beamline of the European Synchrotron Radiation Facility (Grenoble, France). A scheme of the experimental setup can be found in Fig. 5.1. The samples were mounted on a motorized stage and regions of inter-

a

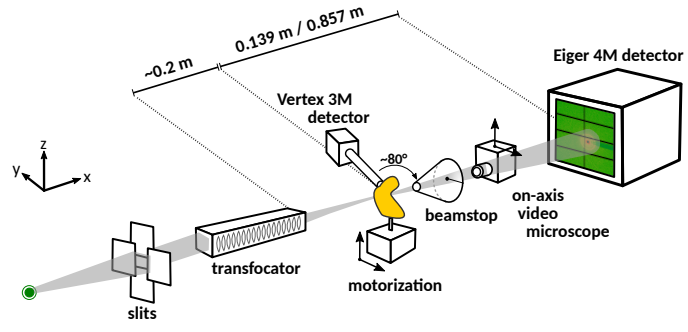

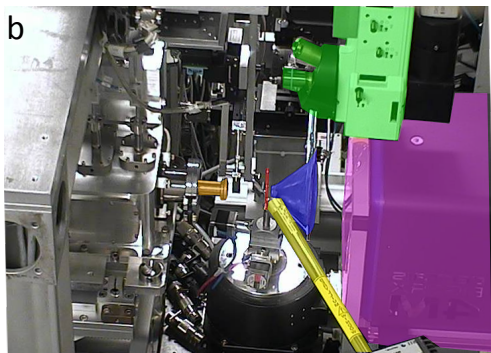

Figure 5.1.: Sketch of the experimental endstation. (a) Monochromatized undulator radiation is focused down to a spot size of $2 \times 3 \mu \mathrm{m}^{2}$ through a transfocator. The sample (tissue section on solid support, SiN window or polypropylene foil) is placed into the focus of the $X$-ray beam. Scattered radiation and fluorescence is detected simultaneously on a $2 D$ pixelated detector (Eiger 4M, Dectris) and Vortex EM detector, respectively. An on-axis video microscope facilitates sample positioning and alignment. A circular beamstop blocks unscattered radiation at approx. $10 \mathrm{~cm}$ downstream of the focus position. A photograph of the setup is shown in (b). Individual components are highlighted in color: Transfocator exit window (orange), sample holder (red), Vortex EM detector (yellow), He-flushed flighttube (blue), on-axis microscope (green), and Eiger $4 \mathrm{M}$ detector (magenta).

est were identified with a movable in-line optical microscope. Diffraction patterns were recorded using an Eiger 4M detector (Dectris, Baden-Daettwil, Switzerland) 
placed either $0.139 \mathrm{~m}$ or $0.857 \mathrm{~m}$ behind the sample to collect data in the wideand small-angle range, respectively. Simultaneously, a Vortex EM detector (Hitachi High-Technologies Science America, Northridge, USA) was positioned in the horizontal plane and tilted at $80^{\circ}$ with respect to the primary beam to record an X-ray fluorescence spectrum at each scan point. The distance of the detector entry window to the sample was $35 \mathrm{~mm}$ and $38 \mathrm{~mm}$, for the SiN mounted tissues and polypropylene mounted tissues, respectively. The energy was set to $12.8 \mathrm{keV}$ defined by the gap of the $\mathrm{Si}(111)$ channel-cut monochromator. Beam-defining slits and a transfocator were tuned to produce an elliptical spot of $2(h) \times 3(v) \mu \mathrm{m}^{2}$. The step size in all scans was therefore set to $3 \mu \mathrm{m}$ to avoid overlap of subsequent scan points. Typically, an acquisition time of $50 \mathrm{~ms}$ was chosen given an overall photon flux of $4.45 \times 10^{11}$ photons $/ \mathrm{s}$.

Immunohistochemistry (IHC) of tissue sections: All chemicals were purchased by Sigma-Aldrich unless otherwise stated. After the synchrotron analyses the tissue blocks were fixated in 4\% PFA (Roth, \#0335) for $48 \mathrm{~h}$ and subsequently embedded in paraffin. Histological evaluations were carried out on $4 \mu \mathrm{m}$ thick sections after deparaffinization [187]. Briefly, the sections were submerged two times for $10 \mathrm{~min}$ in xylene and rehydrated in ethanol solutions of decreasing concentration for 5 min each step: 100\%, 99\%, 96\%, 90\%, 70\% and 50\% ethanol. For each sample, a section was stained with hematoxylin and eosin (HE) according to Mayer's method [188]. Briefly, the sections were put in hematoxylin solution (\#H9627) for $10 \mathrm{~min}$, then washed in water, and differentiated in 1\% $\mathrm{HCl}$-ethanol solution. Then, the slides were left 10 min to bluing in running tap water and finally rinsed in distilled water. The slides were incubated $3 \mathrm{~min}$ in eosin solution made with $0.25 \%$ eosin Y (\#230251), 0.025\% acetic acid (Roth, \#6755) in 80\% ethanol. Subsequently, the slides were washed in distilled water and dehydrated through passages in alcohol with ascending concentration of 70\%, 90\%, 95\%, 99\% and two times $100 \%$ for $3 \mathrm{~min}$, lastly twice in xylene for $5 \mathrm{~min}$. Slides were mounted with mounting medium (\#06522).

The sections stained for alpha-synuclein (aSyn) and p129-aSyn underwent antigen retrieval in citrate buffer made with $10 \mathrm{mM}$ sodium citrate (\#251275), 0.05\% TWEEN 20 (\#P9416) at pH 6.0 for 3 times 5 min in the microwave. After cooling down, the slides for aSyn and myelin were only rinsed in distilled water and the endogenous peroxidases were inactivated in $3 \% \mathrm{H}_{2} \mathrm{O}_{2}$ solution (Applichem, \#121076) in phosphate-buffered saline (PBS) (Applichem, \#A0964) for $10 \mathrm{~min}$. After three washes in PBS, all the slides were blocked for $1 \mathrm{~h}$ in $10 \%$ fetal calf serum (FCS)/PBS (Biochrom, \#S0615). The sections were incubated at $4^{\circ} \mathrm{C}$ overnight 
with the following antibodies: anti-human myelin basic protein (Dako, \#A0623) dilution 1:2000; anti-human aSyn (Covance, SIG-39725) dilution 1:500, anti-p129aSyn (Abcam, \#PS129). After removal of the primary antibody, the secondary biotinylated antibodies were either sheep anti-mouse (Amersham, \#RPN1001) or donkey anti-mouse (Amersham, \#RPN1003) and were diluted in 1:200 and employed at RT for $45 \mathrm{~min}$ for the slides for aSyn and myelin. The revelation was achieved by diluting 1:1000 the avidin-peroxidase (\#A3151) and leaving it in incubation for $45 \mathrm{~min}$. After three rinses in PBS, slides were incubated in $1 \%$ DAB liquid substrate (\#D7304) in PBS with the addition of $0.01 \% \mathrm{H}_{2} \mathrm{O}_{2}$. For p129-aSyn stain, secondary antibody goat anti-rabbit conjugated with alkaline-phosphatase was used (Dako, \#D0477) at RT for 45 min. For p129-aSyn a commercial kit was used for developing (DCS, \#HK183-5K). After developing, all the slides were washed in PBS and counterstained with eosin, dehydrated and mounted as previously described.

\subsection{Results}

X-ray fluorescence results: XRF data was recorded from SN tissue sections with a total scanned area of $6.45 \mathrm{~mm}^{2}$, divided into $2.52 \mathrm{~mm}^{2}$ for the CTR and $3.93 \mathrm{~mm}^{2}$ for the PD sample. From the scanned area, $5.83 \mathrm{~mm}^{2}$ was used for a quantification of the elemental content of SN neurons. Maps of the distribution of single elements were obtained by a simple summation of the fluorescence intensity between the respective channel numbers after calibration of the detector. It was found that the $\mathrm{Zn} \mathrm{K} \alpha$ line showed the most pronounced difference in signal originating from regions identified as intra-cellular and background regions. Using the Zn signal as a cell defining threshold, we have identified $\mathrm{Zn}$ thresholds in each scan for cell identification, as e.g. shown in Fig. 5.2 together with an optical micrograph for comparison. From the optical micrograph in Fig. 5.2(a), dopaminergic neurons are easily discernible because of the presence of the dark pigment NM. Therefore, even without staining, NM positive cells can be identified under an optical microscope. Fig. 5.2 shows that there is a high correlation between the NM positive cells and the area identified by applying a simple threshold to the $\mathrm{Zn} \mathrm{K} \alpha$ map (red contours in Fig. 5.2b).

For all scans, it was possible to separate areas of cellular signal and background. Hence, the summed spectra inside and outside of cells were generated for the PD and CTR samples respectively, and divided by the scanned area, resulting in four spectra which are shown in Fig. 5.3(a). Counts were normalized to the maximum 
intensity given by the elastic scattering peak. Once again, one can observe that the peak of $\mathrm{Zn} \mathrm{K} \alpha$ fluorescence at $8.6 \mathrm{keV}$ is higher for the cells and lower for the background, thus being consistent with our initial assumption. Elemental decomposition of the summed spectrum was carried out using the analysis software pyMCA [189]. Fig. 5.3(b-e) depicts the results separately for intra- and extracellular, as well as for the PD and CTR sample. The presence of Si in the spectrum is due to the use of a Si-drift detector, while $\mathrm{Ar}$ is a major constituent of air. Contributions of $\mathrm{P}, \mathrm{S}$, and $\mathrm{Cl}$ at the lower end of the spectrum are too weak for a quantification, and will not be treated here. From the fitted spectrum, for each elemental component we have determined the variation in the relative concentration by subtracting the area under the elemental distribution from the PD sample from the CTR sample and divided by the area of the PD sample for normalization. Hence, formally, we can write (PD-CTR)/PD. This means that positive values refer to a higher concentration of an element in the PD sample relative to the CTR. All major components are summarized in this fashion in Fig. 5.3(f) along with their respective percentage variation with respect to the control sample.

In the PD sample compared to the CTR, Fe amounts show a clear increase. Relative amounts are, in fact, $90 \pm 12 \%$ in the intracellular space, while there is an increase of $277 \pm 41 \%$ for the same element in the extracellular space. Regarding $\mathrm{Cu}$, there is reduction in the PD sample compared to CTR. The intracellular relative amount of $\mathrm{Cu}$ is $-68 \pm 9 \%$, while it is $-55 \pm 13 \%$ in the extracellular space.
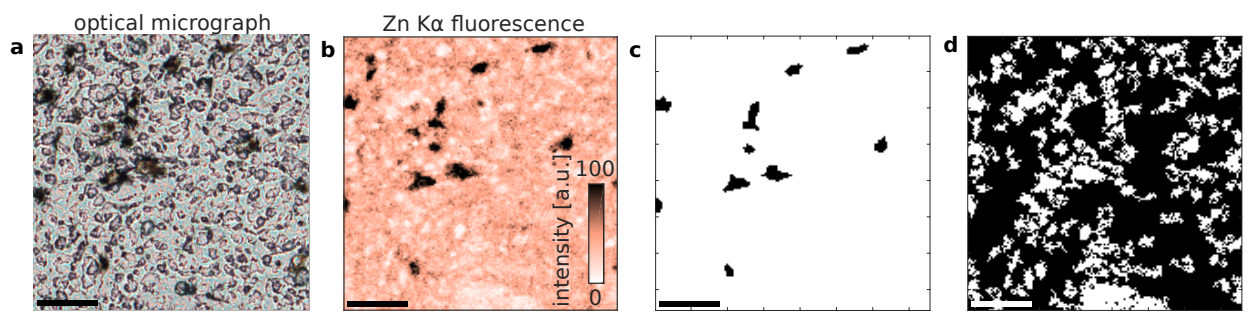

Figure 5.2.: (a) Optical micrograph corresponding to a region of a single scan on $S N$ of PD sample. (b) Corresponding Zn K $\alpha$ fluorescence intensity. Dark regions correspond to NM positive cells in the tissue. (c) Defining a fixed threshold of the $\mathrm{Zn} \mathrm{K \alpha}$ fluorescence yields a logical map to locate the neuromelanin-positive cells (black). (d) A lower threshold yields a logical map corresponding to the extracellular region (black). Scale bar: $100 \mu \mathrm{m}$.

X-ray diffraction results: In addition to the element specific contrast, structural features were analyzed by means of X-ray diffraction. Scattering patterns were collected on a single photon counting 2D pixelated detector (Eiger 4M, 1 pixel 

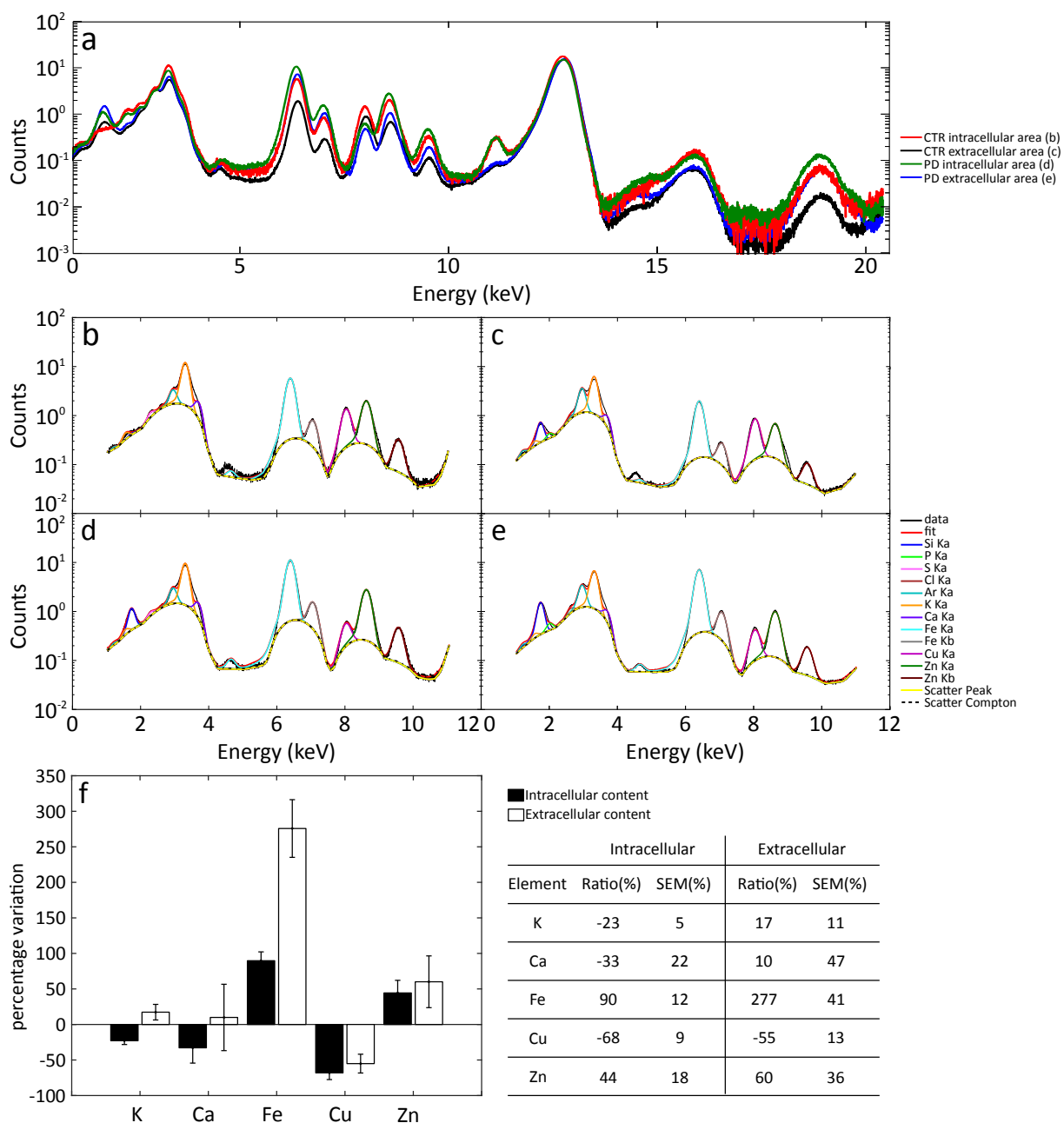

\begin{tabular}{|c|c|c|c|c|}
\hline \multirow[b]{2}{*}{ Element } & \multicolumn{2}{|c|}{ Intracellular } & \multicolumn{2}{|c|}{ Extracellular } \\
\hline & Ratio(\%) & SEM(\%) & Ratio(\%) & SEM(\%) \\
\hline K & -23 & 5 & 17 & 11 \\
\hline $\mathrm{Ca}$ & -33 & 22 & 10 & 47 \\
\hline $\mathrm{Fe}$ & 90 & 12 & 277 & 41 \\
\hline $\mathrm{Cu}$ & -68 & 9 & -55 & 13 \\
\hline $\mathrm{Zn}$ & 44 & 18 & 60 & 36 \\
\hline
\end{tabular}

Figure 5.3.: (a) Comparison of the averaged fluorescence spectra obtained from the cellular and extracellular regions of the PD and CTR sample. Note that cells of the PD sample contain a higher amount of Fe with respect to the CTR. (b-e). Spectral decomposition of the data shown in (a) was done using pyMCA. (b) Spectral decomposition of the intracellular area of CTR. (c) Spectral decomposition of the extracellular area of CTR. (d) Spectral decomposition of the intracellular area of PD. (e) Spectral decomposition of the extracellular area of PD. Meaningful elements were selected for further quantification. (f) Ratios of fitted areas of the elements between PD and CTR. There is a higher concentration of $\mathrm{Fe}$ for the $\mathrm{PD}$ samples especially in the extracellular area, while the $\mathrm{Cu}$ concentration is reduced both in the extra- and intracellular compartment.

point-spread function, Dectris, Switzerland) placed $0.139 \mathrm{~m}$ or $0.895 \mathrm{~m}$ downstream of the focus to collect the diffraction signal in the wide- and small-angle range, 
respectively. With both detector positions, we could accommodate $q_{r}$-ranges between $0.025 \mathrm{~nm}^{-1}$ to $4.1 \mathrm{~nm}^{-1}$, suitable to detect reflections resulting from larger ultrastructures such as fibers, fibrils and lipid bilayer stacks as well as reflections from crystalline aggregations.

First, we analyzed the small-angle scattering signal that we tentatively attribute to the myelin sheath which surrounds the axon of nerve cells and facilitates nerve signal conduction. Myelin is an abundant component of neuronal tissue, however, its concentration varies based on the density of axons and degree of myelination in different brain regions. From the histological staining of myelin basic protein (MPB) shown in Fig. 5.4, it can be seen that the amount of myelin is reduced in the SN due to the high density of cell bodies of dopaminergic neurons that constitute the SN (Fig. 5.4c). On the other hand, the Red Nucleus (Fig. 5.4d) and the Crus Cerebri (CC, caudal region to the SN) (Fig. 5.4b) are richer in axons and thereby myelin, hence they appear darker in the stain.

Based on the hypothesis that the SAXS signal results from the myelin sheath, this difference in density of axons should translate into a higher scattered intensity of the SAXS signal from the CC. In Fig. 5.5(a-d) we show two darkfield maps from SN and fiber tract (FT) tissue from the CC. Data resulting from a homogeneous region in (a) and (c), marked roi 1 and roi 2, respectively, have been averaged and azimuthally integrated, as shown in (b) and (c). Two structure factor peaks are observed in both cases. Importantly, and supporting the myelin hypothesis, the reflection intensity recorded in the FT is stronger, by a factor of at least two. Further, the ratio of the two reflections is found to be $3 / 2$. It is therefore plausible, that the inner and outer peak indicate a second and third order of a structure with a period of approximately $12.8 \mathrm{~nm}$. In turn, this corresponds well to periods reported for myelin nerves that have been dried to prepare the sample for electron microscopy [190]. The observed peak positions and peak widths are summarized in Tab. 5.1. In addition, one should expect a more oriented alignment of the myelin signal in a region rich in neuronal connections due to the directed nature of neuronal connections linking different parts of the brain. To confirm this, we have quantified the level of anisotropy of the myelin diffraction signal by performing a principal component analysis of the diffraction patterns, as previously introduced in [130]. In this approach, the covariance matrix of the distribution of the wavevector components $q_{y}$ and $q_{z}$ in the detection plane, defined as

$$
C=\left(\begin{array}{cc}
\operatorname{var}\left(q_{y}\right) & \operatorname{cov}\left(q_{y}, q_{z}\right) \\
\operatorname{cov}\left(q_{z}, q_{y}\right) & \operatorname{var}\left(q_{z}\right)
\end{array}\right)
$$


Imaging of neuronal tissues by X-ray diffraction and X-ray 90 fluorescence microscopy: evaluation of contrast and biomarkers for neurodegenerative diseases

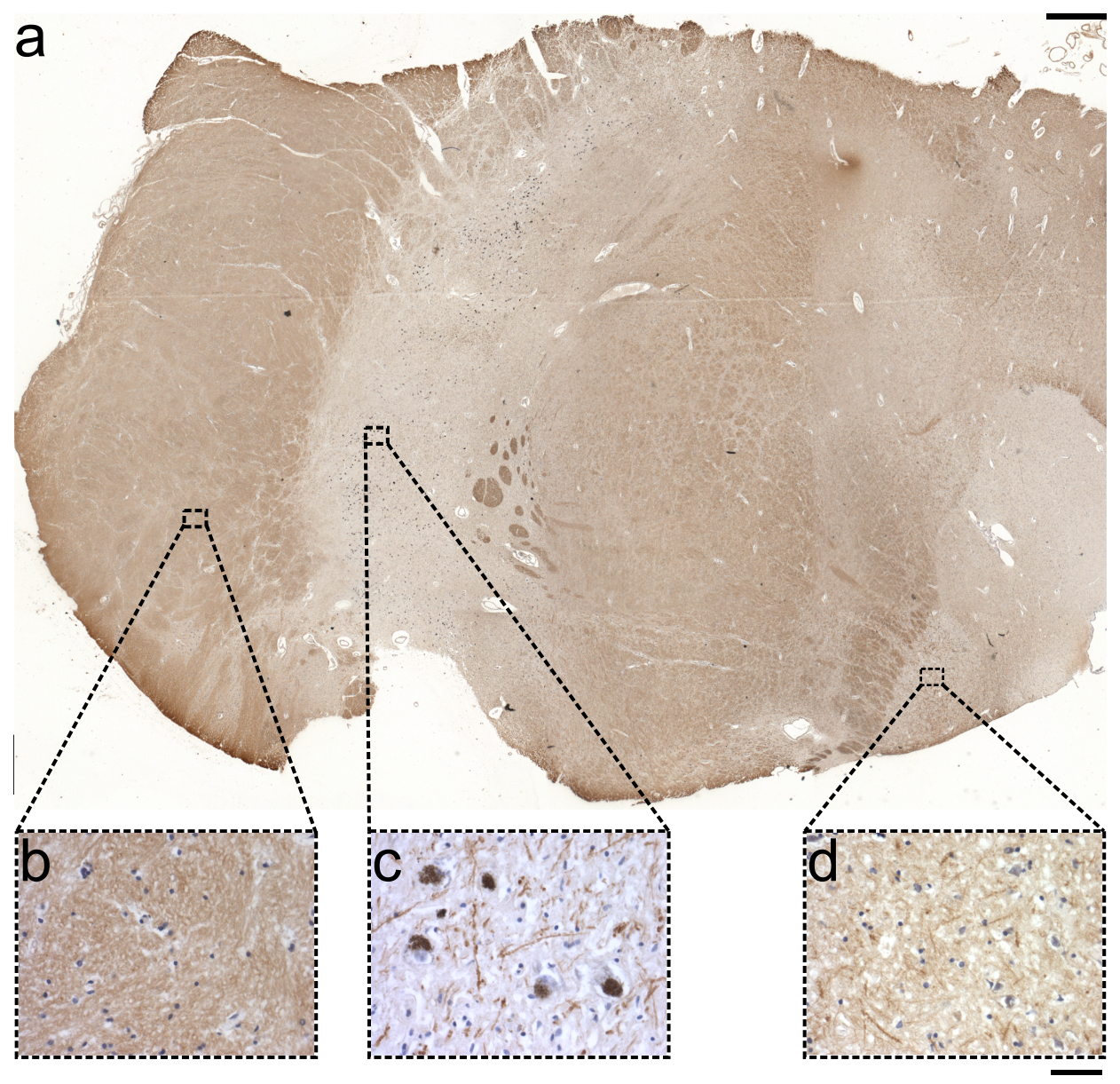

Figure 5.4.: (a) Overview of an IHC staining of MBP from tissue of the CTR sample in a neighboring section of those that have been investigated using $X$-rays. The staining is resulting in a brown color due to the product of the reaction between horseradish peroxidase and $D A B$. The intensity of the color is dependent on the amount on MPB present in the region. Scale bar: $500 \mu \mathrm{m}$. (b-d) Close-up regions show the myelin content of (b) Crus cerebri, (c) Substantia Nigra, and (d) Red Nucleus. Scale bar: $50 \mu \mathrm{m}$.

is diagonalized. The two corresponding eigenvectors $\vec{v}_{1}$ and $\vec{v}_{2}$ now form an orthogonal basis with $\vec{v}_{1}$ indicating the principal direction of scattering. The variance in each direction is given by the eigenvalues $\lambda_{1}$ and $\lambda_{2}$. In this respect, the anisotropy of the scattering can conveniently be quantified by the unitless parameter $\omega$ defined as

$$
\omega=\frac{\left|\lambda_{1}-\lambda_{2}\right|}{\lambda_{1}+\lambda_{2}} .
$$


Note that prior to the PCA analysis, one typically has to define a range of q-vectors, here an annulus of the diffraction pattern, of which the anisotropy is computed [130]. We found that indeed, the anisotropy in the Crus Cerebri region is high and an orientation can be clearly deduced from the diffraction, as visualized in Fig. 5.5. The orientation in Fig. 5.5(b) is coded in color as well as by arrows indicating the nerve fiber orientation (orthogonal to the direction of $\vec{v}_{1}$ ) in the Crus Cerebri. The anisotropy of the diffraction reported here is based on the diffraction from the second order of the myelin signal, as shown in Fig. 5.6(b), which is more anisotropic in contrast to the third order [see e.g. Fig. 5.6(b)]. In a similar fashion, we have computed the orientation and anisotropy of the third order of the myelin signal (data not shown), which showed that the orientation of the second and third order of the myelin signal are clearly correlated, with the anisotropy of the third order being far less pronounced.

In addition to the anisotropy and orientation reported above, further structural quantities can be extracted from the myelin signal, in particular lamellar repeat spacing and peak width. The spatial fine structure of this signal could be of interest for future studies addressing in particular the lamellar structure of myelin and related pathologies. In this work, however, we want to draw the attention to a particularly surprising finding: apart from the predominant lamellar signal of the myelin sheath, we also found isolated spots in the tissues of both PD and CTR, where the diffraction pattern exhibited a hexagonal symmetry. This is exemplified in Fig. 5.6, where next to a typical lamellar pattern we also present a diffraction pattern with hexagonal symmetry, along with the associated locations in the darkfield map (indicated by arrows). From the fact that higher order reflections are not visible in the diffraction signal, we infer that the underlying lipid arrangement is rather hexatic than strictly hexagonal, i.e. exhibits directional but no positional long range order. To our knowledge, such a phase behavior of the myelin sheath has so far only been observed in in vitro model systems mimicking the chemical composition of the myelin sheath [191] but has not been observed previously in situ in studies employing human tissue, even though an hexagonal phase was already proposed as early as 1963 [192]. To further quantify the hexagonal scattering, we have averaged all patterns exhibiting clear hexagonal symmetry as well as patterns in the bulk of the tissue. For both averaged patterns, we have performed an azimuthal integration to once again determine the peak positions and peak widths. Again, we find that in the bulk, the ratio of the lamellar positions of the second and third reflection order is given by the ratio $3 / 2$. The reflections of the hexatic phase are found at $q_{r}^{(\text {hex })}=1.43 \mathrm{~nm}^{-1}$ corresponding to a period 
a
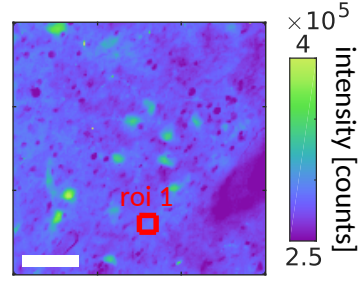

C

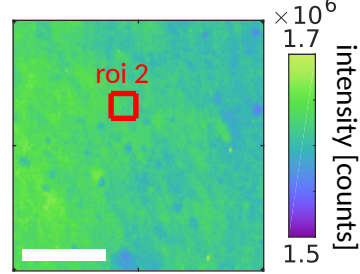

b

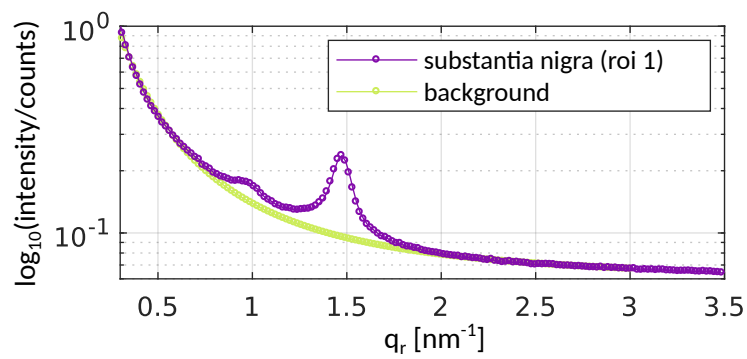

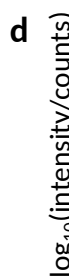

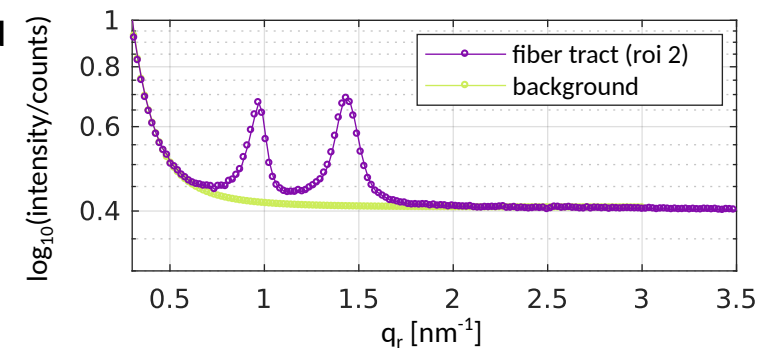

e

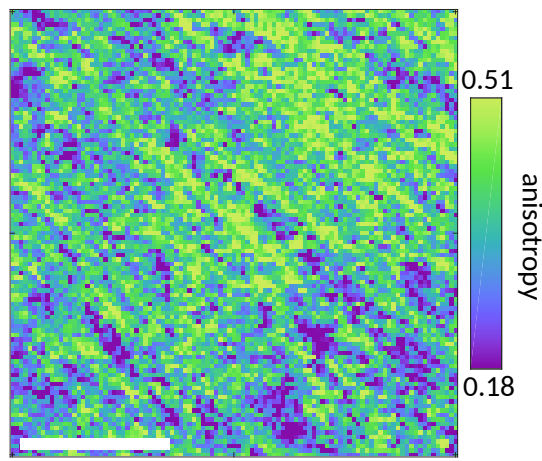

f

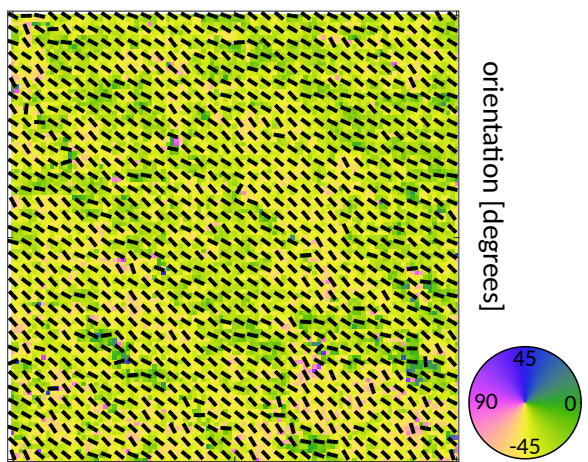

Figure 5.5.: (a-d) Structure factor analysis of the SAXS signal. (a) Darkfield map of SN tissue from CTR sample. Data from region 1, marked in red, was averaged and azimuthally integrated, as shown in (b). (c) Darkfield map of FT tissue from CTR sample. Again, data from region 2, marked in red, was averaged and azimuthally integrated, as shown in (d). (e-f) Principal component analysis of fiber tract tissue of the Crus Cerebri. (e) Anisotropy of the myelin diffraction. (f) Orientation of the long axis of the myelin sheath. Orientation of the axons is both color coded and indicated by superimposed black lines. Scale bar: $100 \mu \mathrm{m}$.

of $a_{H}=\frac{4 \pi}{\sqrt{3} q_{r}^{\text {(hex) }}}=5.1 \mathrm{~nm}$ which is well on the order of the thickness of a single bilayer. The peak positions and peak widths are summarized in Tab. 5.1.

In summary, we hypothesize that the myelin sheath, which would normally be a lamellar stack of lipid membranes surrounding the axon of the neuron as sketched in Fig. 5.6(d) forms an inverse hexatic phase, as shown on the right. Clearly, the 
presence of an hexatic phase could result from the rather invasive sample preparation. The effect of temperature, freezing and drying has been extensively studied in the literature, see e.g. [51, 190, 192-194] and references therein, however, the values reported in the references cannot be directly compared to the values reported here, primarily because the extracted nerve was typically re-immersed in a buffer solution after freezing or heating. Therefore, future extension of this work has to investigate whether myelin is indeed capable of forming hexatic phases.

a
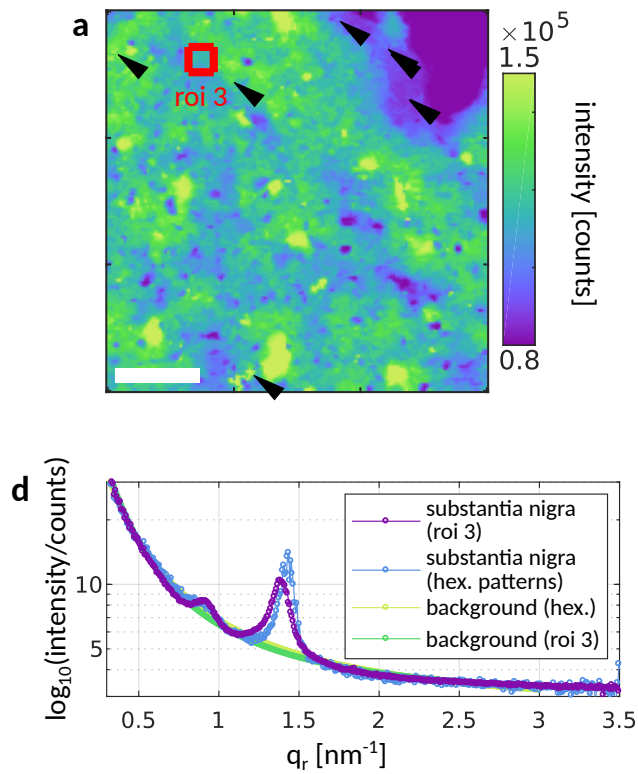
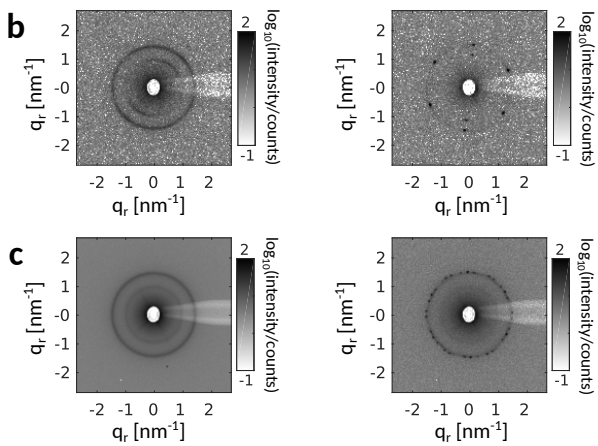

e

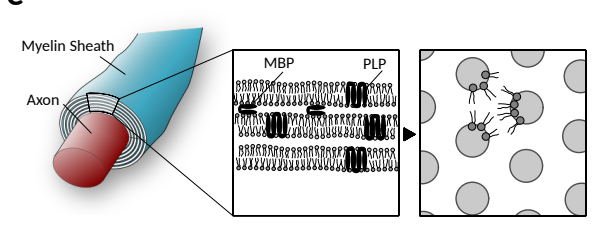

Figure 5.6.: (a) X-ray raster scan (darkfield contrast) of a brain tissue section (SN region) from a Parkinson's disease patient. Arrows indicate locations at which an hexatic lipid phase could be identified. Scale bar: $100 \mu \mathrm{m}$. (b, left) Myelin signal as can be observed throughout the scanned sample region, while ( $b$, right) a signal resembling an hexatic lipid bilayer phase can only be found at isolated locations in the sample, as marked by arrows in (a). (c, left) Averaged signal from roi 3 as marked in red in (a). (c, right) Averaged signal from locations where an hexatic phase was identified. (d) Both patterns in (c) have been angular averaged to identify the peak locations and peak widths. (e) Sketch of the myelin sheath surrounding the axon of a nerve cell. A zoom region shows a sketch of the lamellar stacking of lipid bilayers in the myelin sheath with myelin basic protein $(\mathrm{MBP})$ and proteolipid protein $(P L P)$ as major protein constituents of the myelin sheath. Based on the data it can be speculated that the myelin sheath can undergo a phase transition into an inverse hexagonal phase, as shown on the right. MBP: Myelin basic protein, PLP: Myelin proteolipid protein. Subfigure (e) with adaptions from [195].

While myelin is an abundant component of neuronal tissue, by accessing the wideangle scattering range, smaller crystalline components can be localized by scan- 
Table 5.1.: Summary of peak positions and peak widths as determined from the respective structure factors shown in Fig. 5.5 and Fig. 5.6.

\begin{tabular}{llllll} 
& $q_{r}^{(2,0)}\left[\mathrm{nm}^{-1}\right]$ & $q_{r}^{(3,0)}\left[\mathrm{nm}^{-1}\right]$ & $\sigma^{(2,0)}\left[\mathrm{nm}^{-1}\right]$ & $\sigma^{(3,0)}\left[\mathrm{nm}^{-1}\right]$ & $q_{r}^{(3,0)} / q_{r}^{(2,0)}$ \\
\cline { 2 - 6 } ROI 1 & 0.98 & 1.47 & 0.09 & 0.09 & 1.50 \\
ROI 2 & 0.96 & 1.44 & 0.08 & 0.10 & 1.50 \\
ROI 3 & 0.93 & 1.39 & 0.09 & 0.08 & 1.49 \\
\hline Hexatic phase & $q_{r}^{(\text {hex })}\left[\mathrm{nm}^{-1}\right]$ & 1.43 & $\sigma^{(\text {hex })}\left[\mathrm{nm}^{-1}\right]$ & 0.06 & \\
\hline
\end{tabular}

ning micro-diffraction. Indeed, pronounced wide-angle reflections from crystalline domains where observed in the present samples. In the following, we aimed at localizing these crystalline domains and determining their origin (Fig. 5.7). Localization was achieved in three steps: First, background was subtracted from each diffraction pattern. Secondly, crystalline reflections were identified in each diffraction pattern by applying a threshold of 10 counts (Fig. 5.7b). Thirdly, intensity of the reflections above $1.6 \mathrm{~nm}^{-1}$ and localized in the previous step was integrated. Intriguingly, following this procedure, we noticed that the PD patient sample contained approximately ten times more crystallites than the CTR patient sample (Fig. 5.7d).

To determine the origin of such diffraction, we have estimated the unit cell parameters from diffraction patterns originating from single crystalline domains. The unit cell parameters were referenced against the Cambridge Structural Database (CSD). Anhydrous cholesterol appeared the closest match to the given unit cell parameters. To verify that cholesterol had indeed crystallized primarily in the PD sample, we performed a maximum projection of a data set containing larger amounts of crystallites. In a maximum projection of a stack of $2 \mathrm{D}$ diffraction patterns, the maximum value was calculated for each pixel along the stacking dimension, to accumulate all reflections in the acquired data onto a single $2 \mathrm{D}$ diffraction pattern. In a second step, the maximum projection was angular averaged to give a onedimensional representation taking into account all reflections occurring within the scan [blue curve in Fig. 5.7(e)]. Finally, reflection positions [red lines in Fig. 5.7(e)] were simulated using the values reported in [196] for the unit cell of anhydrous cholesterol and superimposed onto the one-dimensional (powder averaged) data. Data and simulation agree well up to approximately $6 \mathrm{~nm}^{-1}$ where the degeneracy of the triclinic lattice spacing $d_{h k l}$ is low, hence making it highly plausible that the crystallites were indeed formed by anhydrous cholesterol. 

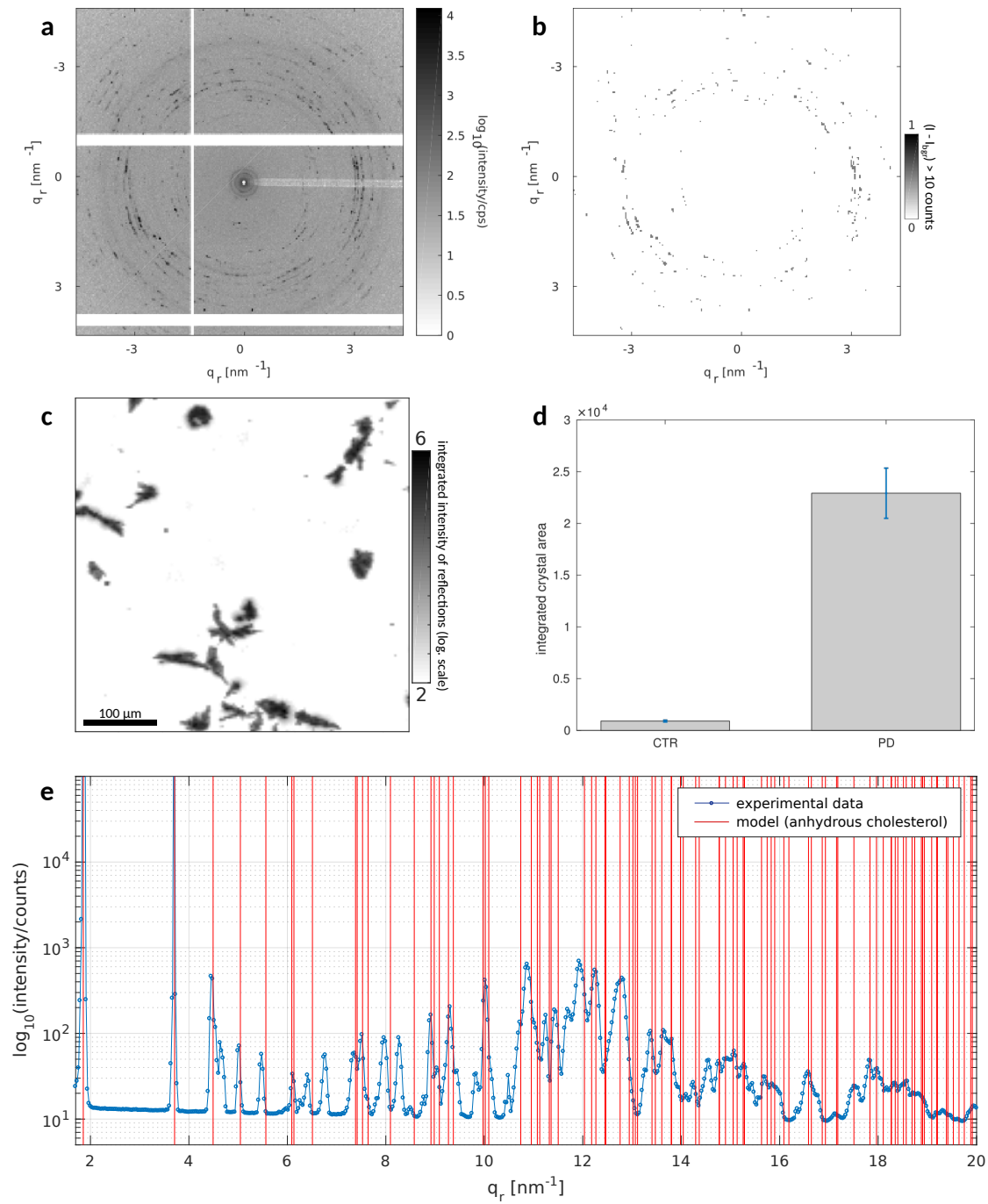

Figure 5.7.: Crystalline reflections from anhydrous cholesterol. (a-c) Illustration of the crystal localization procedure. (a) Diffraction pattern showing the presence of crystalline diffraction peaks in the wide-angle region. (b) Thresholded diffraction pattern (background subtracted) for a threshold of 10 counts. (c) Summing the intensity of the reflections identified by the logical masking procedure in (b) for each scan points yields a map of the crystalline domains of the sample. (d) Summed number of scan points exhibiting crystalline reflections within the CTR and PD sample. (e) Azimuthal integration of the maximum projection of the scan (blue lines and dots) with red lines superimposed corresponding to reflections that would result from anhydrous cholesterol. 
Imaging of neuronal tissues by X-ray diffraction and X-ray 96 fluorescence microscopy: evaluation of contrast and biomarkers for neurodegenerative diseases

Subsequently, we correlated the spatial distribution of cholesterol crystals with a $2 \mathrm{D}$ raster image [darkfield contrast, Fig. 5.8(a)], and optical micrographs (Fig. $5.8 \mathrm{~b}$ ), as well as $2 \mathrm{D}$ maps of the X-ray fluorescence signal, namely the summed fluorescence intensity (Fig. 5.8d) and the $\mathrm{Zn}$, Fe and $\mathrm{Cu}$ fluorescence maps (Fig. 5.8d-f). It can be observed that cholesterol crystals do not co-localize with the distribution of SN neurons and that the cholesterol crystals appear in all cases to be mostly situated in the extracellular space.
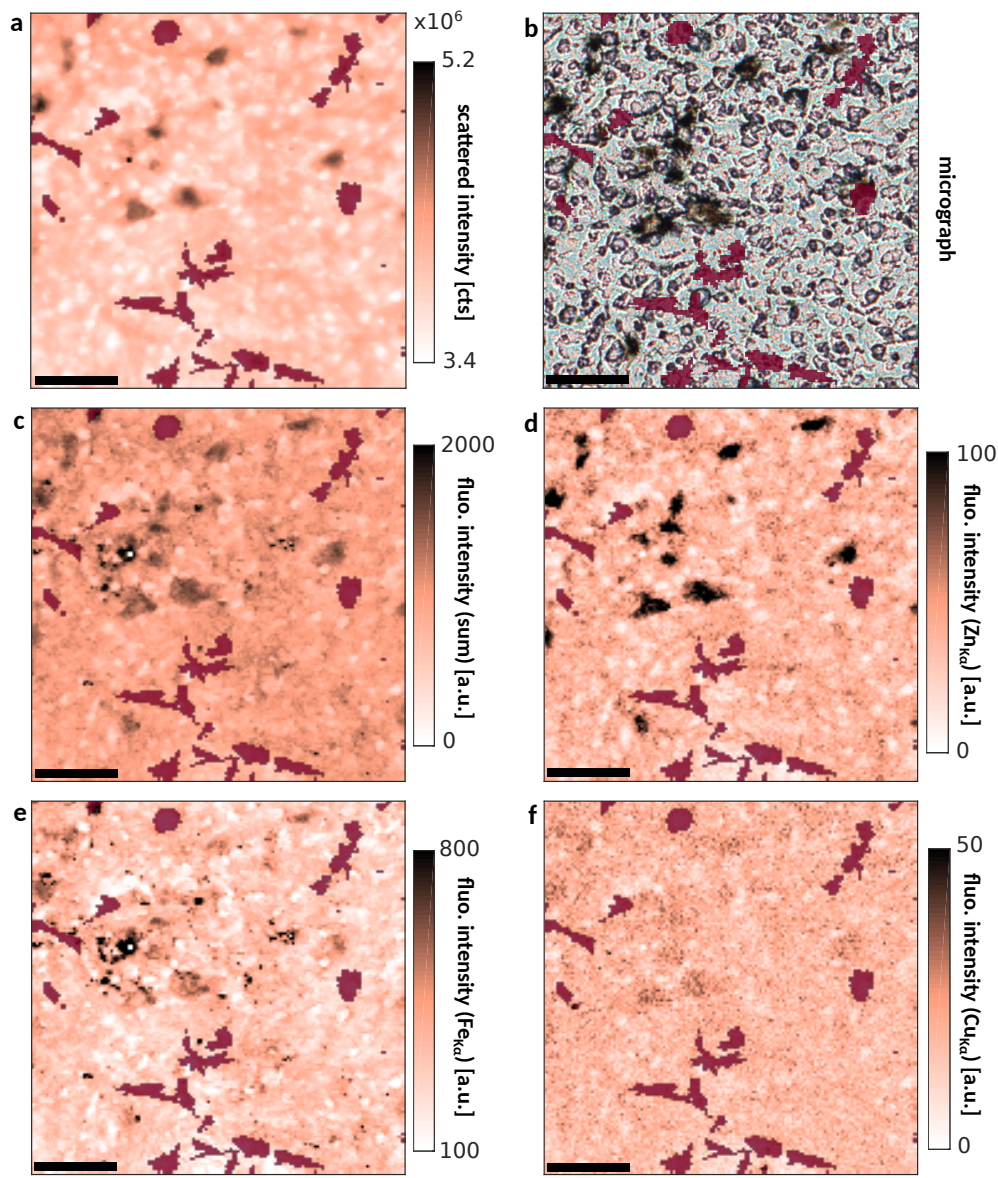

Figure 5.8.: Semitransparent map of the distribution of crystalline aggregates (red areas) overlaying (a) the STXM map (darkfield contrast), (b) optical micrograph, (c) total

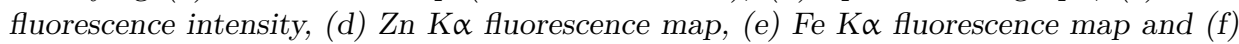
$\mathrm{Cu} \mathrm{K \alpha}$ fluorescence map. Scale bar: $100 \mu \mathrm{m}$.

Histological results: The tissue blocks were embedded in paraffin and the tissue sections were stained with hematoxylin-eosin for a morphological evaluation and 
also with two different antibodies: anti-aSyn and anti-p129-aSyn. In the PD sample, HE-stain showed the presence of intracellular aggregates that were identified as Lewy bodies (LB, Fig. 5.9, top) and no other kinds of aggregates were found. On the other hand, the CTR sample was negative for aggregates. The LB found were mostly present in the intracellular space and their diameter was ranging from 2 to $15 \mu \mathrm{m}$.

To confirm that the intracytoplasmic inclusions that resembled LB contained aSyn, the sections were stained with antibodies against aSyn and its phosphorylated form, which is mostly found in LB. Both antibodies confirmed the presence of LB solely in the PD sample.

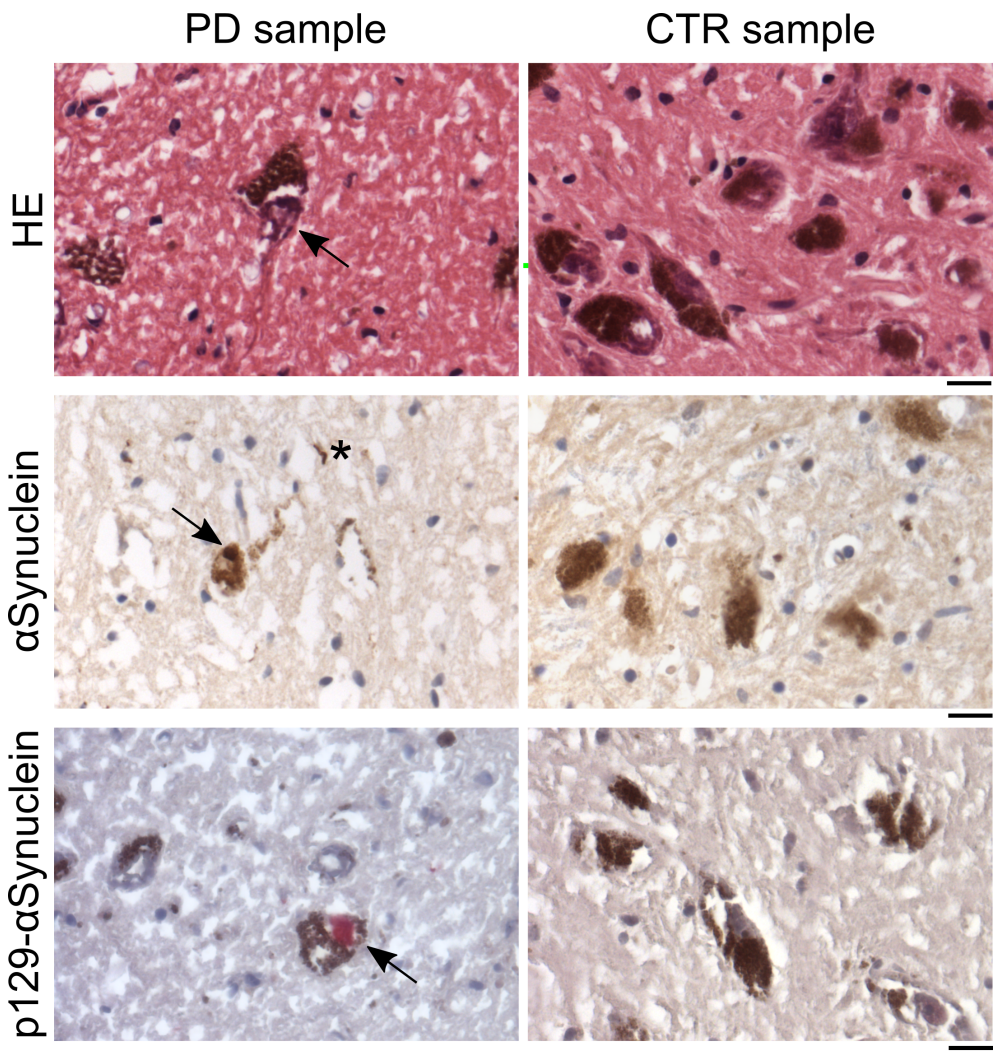

Figure 5.9.: Histological evaluations of the samples. Each row represents the primary antibody used, both for PD (left column) and CTR (right column). The arrows point to Lewy bodies and the star highlights a Lewy neurite. Scale bar: $20 \mu \mathrm{m}$. 


\subsection{Discussion and outlook}

In our experiment, we have combined scanning X-ray fluorescence and X-ray diffraction to map neuronal tissues with high spatial resolution. We chose SN of a PD patient as well as a control, aiming at a translation of advanced structural techniques to biomedical research in $\mathrm{PD}$, and more generally in neurodegeneration. We found that the scanned PD tissue displayed a well-documented metal dyshomeostatsis [176, 197]. Our data are in agreement with previous studies, which showed that the highest amount of metals on the human SN are contained inside the neurons while in the extracellular space lower concentrations are reported $[176,198]$. The entire elemental spectra of all scans were analyzed to identify a signal suitable to discriminate between intracellular and extracellular space. The neurons analyzed in SN are mostly NM positive cells, and previous reports pointed out that NM is rich in Fe, $\mathrm{Cu}$ and $\mathrm{Zn}$ [199]. Among these elements, Zn was the element that displayed the greatest concentration difference between intraand extracellular content. Therefore, a threshold on the $\mathrm{Zn}$ signal was chosen for automated identification of the two regions in the form of a logical mask. Using these masks for all scans, four different spectra were computed: intracellular and extracellular (sum) spectrum for PD, as well as intracellular and extracellular (sum) spectrum for the CTR sample.

Compared to the CTR, Fe amounts were found to be three times higher in the extracellular space for the PD tissue. This is consistent with results from other groups [200]. Intriguingly, the Fe distribution in the extracellular space does not seem to follow a regular pattern. Indeed, bright spots of Fe appear frequently in scans. The reason for the presence of these irregular Fe deposits deserves further investigation. The methods followed in sample preparation (like the special cryomatrix used, the temperature for sectioning and the special Teflon-coated blades) should have prevented the displacement of metal between the inside and outside of the cell. The extracellular Fe depositions might thus indicate some still unexplored mechanism in the pathology. $\mathrm{Cu}$ concentration in the $\mathrm{PD}$ patient, on the other hand, are reduced by about $50 \%$ in both the intracellular and extracellular space. This is in line with a recent report [197]. The analysis of metal content in tissue is further motivated by the fact that in vitro experiments indicate that the interaction of aSyn with several metals (like $\mathrm{Fe}$ and $\mathrm{Cu}$ ) promotes its aggregation [201-203].

Hematoxylin-eosin staining is routinely performed in the course of histological evaluations as it provides a morphological characterization of the tissue [187]. In the 
PD sample analyzed we could detect LBs by HE staining as they appeared with a dense eosinophilic core surrounded by a clearer halo. The stain with aSyn and p129-aSyn confirmed the presence of LBs and of Lewy neurites. All in all, the results are in line with 'classic' PD histology, while the control sample was devoid of these features. Even though LBs were identified by histological staining of adjacent sections, we were unable to locate LBs during the scanning diffraction experiment, which is a challenging task given their low number and small size ranging between $2 \mu \mathrm{m}$ and $6 \mu \mathrm{m}$ [174]. It would be an important future goal to elucidate the molecular structure of the LB by X-ray micro-diffraction, using the scanning approach presented here. A similar approach using tissue from Alzheimer's diseased patients has already proven successful in providing useful insights on the development of amyloid plaques [204].

Contrarily, we found a pronounced diffraction signal corresponding to highly ordered crystalline structures which formed extended domains, primarily in the PD sample, and could show that the crystallites are formed by cholesterol. The formation of cholesterol crystals could have been caused by the fact that the tissue was left to dry at $60^{\circ} \mathrm{C}$, which might have triggered a phase transition, typically occurring during cooling of the sample at around $44^{\circ} \mathrm{C}$ [205]. However, cholesterol crystals also seem to occur in preparations of nervous tissue where the sample was dried at temperatures lower than $44^{\circ} \mathrm{C}$ [198]. The fact that crystallization took primarily place in the $\mathrm{PD}$ sample could be explained in at least two ways. One is that the amount of cholesterol in the PD sample was higher than the control. A lipidomic study is in support of this (first) hypothesis. In fact, it has been reported that in the visual cortex of PD patients there is an increased cholesterol level compared to control patients [206]. The other explanation is that the proportion of the different phospholipids and cholesterol in the membrane was different between the patient and control. This could lead to a lowering of the phase transition temperature thus facilitating the phase change of cholesterol with consequent cholesterol crystal formation. This hypothesis is also supported by a study in which the author could correlate the variation of phospholipid composition in the membrane to its damage in PD patients [207].

Either way, cholesterol and several oxysterols have been correlated with their role in aging and in neurodegenerative pathologies such as Alzheimer disease [208]. The blood brain barrier naturally occurring in the brain prevents cholesterol and its derivatives to enter the brain from the blood stream. Astrocytes produce most of the cholesterol in the brain. Variations of cholesterol content are related to metabolic dysfunctions in the pathway connected to this lipid [181]. Animal studies 
in mice correlate a diet rich in fat or cholesterol with the worsening of neurotoxinsinduced loss of dopaminergic neurons [209]. In in vitro studies, aggregated forms of aSyn could disrupt phospholipidic bilayers that where rich in cholesterol [210]. Regarding cholesterol in PD, one should also keep in mind the role of oxysterols. These are oxidized forms of cholesterol. They have exactly the same structure of cholesterol with the addition of one (or more) hydroxyl groups on the sterol nucleus or on the side chain. They are chemically very similar to their cognate and they arise from the ROS produced in the cells that are particularly abundant in neurodegenerative diseases such as PD. These molecules have been found to be in excess in PD patients and they are also suggested to be involved in the etiopathology of PD [181].

Besides having identified and localized cholesterol as tissue component, XRD scanning of the tissue also revealed a pronounced anisotropic SAXS signal, which can be attributed to myelin. The orientation and the peak spacings of the myelin sheath can thereby be mapped throughout the tissue, quantifying the underlying axonal orientation. Interestingly, it was found that in sparse locations through the sample, the myelin scattering suddenly became hexagonal with no long-range correlation, pointing at a hexatic phase of myelin at these spots. Future work will have to corroborate this finding and verify whether it is intrinsic or results from an invasive sample preparation.

In conclusion, in our study we have combined standard histological techniques with advanced spatially resolved XRD and XRF. A pronounced difference was found in trace metal composition of the PD sample compared to the control. In addition, a crystalline diffraction signal attributed to cholesterol was observed to be more abundant in the PD tissue. Given the exiguity of patients, the biological findings need to be further characterized in a larger cohort. Overall, the approach described here for the characterization of human tissue is new and promising for future applications such as the discovery of new biomarkers for neurodegenerative diseases.

\section{Funding}

Deutsche Forschungsgemeinschaft (DFG) (EXC 171, SFB 937). 


\section{Acknowledgments}

The authors thank Elisabeth Barski for expert technical assistance and Manfred Burghammer for excellent working conditions at beamline ID13.

\section{Disclosures}

The authors declare that there are no conflicts of interest related to this article. 



\title{
6. The optical stretcher as a tool for single-particle $\mathrm{X}$-ray imaging and diffraction
}

\author{
J.-D. Nicolas, J. Hagemann, M. Sprung and T. Salditt
}

Reproduced from Journal of Synchrotron Radiation 25, 1196 - 1205 (2018).

For almost half a century, optical tweezers have successfully been used to micromanipulate micrometer and sub-micrometer-sized particles. However, in recent years it has been shown experimentally that, compared with single-beam traps, the use of two opposing and divergent laser beams can be more suitable in studying the elastic properties of biological cells and vesicles. Such a configuration is termed an optical stretcher due to its capability of applying high deforming forces on biological objects such as cells. In this article the experimental capabilities of an optical stretcher as a potential sample delivery system for X-ray diffraction and imaging studies at synchrotrons and X-ray free-electron laser (FEL) facilites are demonstrated. To highlight the potential of the optical stretcher its micromanipulation capabilities have been used to image polymer beads and labeled biological cells. Even in a non-optimized configuration based on a commercially available optical stretcher system, X-ray holograms could be recorded from different views on a biological cell and the three-dimensional phase of the cell could be reconstructed. The capability of the setup to deform cells at higher laser intensities in combination with, for example, X-ray diffraction studies could furthermore lead to interesting studies that couple structural parameters to elastic properties. By means of high-throughput screening, the optical stretcher could become a useful tool in X-ray studies employing synchrotron radiation, and, at a later stage, femtosecond X-ray pulses delivered by X-ray free-electron lasers. 


\subsection{Introduction}

X-ray structural analysis at the level of a single particle has become a reality with the advent of highly brilliant synchrotron and X-ray free-electron laser (FEL) radiation [70, 211-213]. Following a hundred years of studying matter by X-ray diffraction in terms of macroscopic ensemble averages, we now have intense focused beams at hand to probe an individual object, separately out of an ensemble. This opens up a unique opportunity to address the polydispersity and polymorphism of soft and biological matter, and to study structure in complex hydrated environments without losing information by ensemble averages. Application examples range from colloidal particles, viruses [160] and lipid vesicles to bacteria [161] and eukaryotic cells. To circumvent radiation damage, 'diffract before destroy' or 'diffract before damage' strategies have to be implemented. To this end, samples have to be replenished continuously, and a sufficiently high number of copies have to be probed consecutively. Successive exposures, each time on a new non-identical element of a set, are acquired. Analysis of the entire distribution of structural observables then provides substantially more information than the conventional ensemble average, i.e. the classical powder and population average. For identical particles, the distribution over which the acquisitions are sampled does not refer to the structure but to the orientation angles. A full dataset for threedimensional (3D) structure analysis then requires many different views, where each can be sparse in photon counts.

While the potential of single-particle diffraction and imaging in particular on identical particles is undisputed, a tremendous challenge is in realizing suitable sample delivery tools, enabling hydrated environments, precise positioning of the object in the beam, sufficient throughput and hit rate. While liquid jet and droplet technology (aerosol jets) are already quite advanced and have paved the way for studies of smaller, identical particles such as viruses wrapped in a thin hydrated shell [160, 214], sample delivery of non-identical particles such as larger colloids, bacteria or eukaryotic cells $[162,163]$ require a different approach, both in terms of cross section of the delivery system as well as in view of a need for pre-screening of the object, and eventual rotation and positioning. To this end, channel microfluidics can be made compatible with X-ray experiments using suitable thin foil window materials, as reviewed in [165] and [166]. As far as trapping, positioning and rotation are concerned, optical forces are the tool of choice to manipulate an individual particle, for example in a channel with or without flow, and to prevent the particle or cell from diffusing out of focus. 
For this reason, optical tweezer technology as introduced by Ashkin [215-217] has already received significant attention, and X-ray analysis of trapped particles has been demonstrated for different applications. Examples include diffraction of multilamellar liposomes trapped in an optical tweezer [218], and starch granules which have even been studied with full control of 3D orientation in [219] and also in $[220,221]$. Coherent diffraction imaging (CDI) experiments on particles trapped in an optical tweezer have been reported [222]. Single-molecule optical trapping and small-angle X-ray scattering studies have been reported in [223]. Finally, Xray fluorescence measurements of microalgae trapped by optical tweezers have been reported [224, 225]. The disadvantage of optical tweezers is that the trapping potential is not steep enough to avoid significant motion of the particle. This becomes possible, however, by resorting to the original version of an optical tweezer suggested by Ashkin, which was based on two unfocused counter-propagating laser beams. This configuration, as demonstrated experimentally by Guck and Käs [226], can be used to arrest a biological cell at typical laser powers of $100 \mathrm{~mW}$, and even to stress cells for mechanical testing at typical powers of $1 \mathrm{~W}$, excerting forces of the order of $100 \mathrm{pN}$. In a ray optical picture the surface stress arises by momentum transfer when a laser photon with $p=h n / \lambda$ increases its momentum by entering a medium with higher index of refraction $n$ than its surrounding medium. Alternatively, one can account for the stretching force by calculation of the Maxwell stress tensor [227, 228]. The microfluidic chip design for the dual-beam optical trap and stretcher was first described in [229], and more recently the effect of a trapped particle on the beam propagation has been studied in [230]. Apart from cell screening, previous applications of the optical stretcher have also addressed phospholipid assemblies [231] and stretching of giant unilamellar vesicles (GUV) [232].

In this work, we now demonstrate that the optical stretcher is a well suited tool for single-particle X-ray studies, given its ability to manipulate and rotate a nonadhering biological cell in a flow environment. We show this here for the example of full-field holographic imaging using synchrotron radiation. We first demonstrate the procedure based on polystyrene beads, before we turn to labeled macrophage cells, where we can even show that controlled rotations provide different views for tomographic analysis. Note that in most current studies of cellular imaging, not only by X-rays, cells are imaged while adhering to substrates or are embedded in an extracellular matrix. However, there are also a number of suspension cell types, such as blood cells and many bacteria and other unicellular organisms, where the physiological state of interest is in a suspension. Furthermore, some cases may 
require the use of fluid flow as an additional control parameter, or even elastic deformation, the application for which the stretcher was devised originally. Note that, based on the technical developments over the last decade, the stretcher has become a screening tool for cell elasticity with statistical throughput rates of about 250 cells per hour. In this work we propose to use this technology for single-cell X-ray studies using high-brilliance synchrotron radiation and FELs. To this end, we take a commercial stretcher with almost no special optimization for the Xray probe and place it in the nano-focus setup GINIX at the PETRA III storage ring [63]. Even without any further optimization (thin foil windows, background reduction) the results already prove the concept.

The article is structured quite simply in the following way. Following this introduction, the experiment is described in Sec. 6.2 along with the associated methods, including the stretcher and the integration into the beamline. Sec. 6.3 then presents the results obtained for polystyrene beads and macrophage cells, before the article closes in Sec. 6.4 with a brief conclusion and outlook.

\subsection{Experiment}

\subsubsection{Optical stretcher system}

In the following, we briefly review the essential components of an optical stretcher. For a more in-depth description of the optical stretcher, see [233] and [229].

The optical stretcher system (Optical Stretcher, RS Zelltechnik GmbH, Leipzig, Germany) is composed of a microfluidic sample chamber, a laser for cell trapping as well as a height leveling system and an air pressure pump for flow control. The infrared (IR) laser system can be used to trap or stretch cells in the microfluidic channel. We have used a fiber laser [Fibolux V3.1 (Yb-2x33), FiboTEC, Meiningen, Germany] with an exit wavelength $\lambda$ of $1060 \mathrm{~nm}$ and a maximum continuous wave output power of $1.2 \mathrm{~W}$ from two seperate single-mode fibers. The minimum emission power is $50 \mathrm{~mW}$. For the trapping experiment, we have used an output power between $70 \mathrm{~mW}$ to $150 \mathrm{~mW}$ per fiber. The two output channels of the laser are fiber-optically coupled to the microfluidic sample chamber where it is integrated into a microfluidic chip. The glass fibers with an exit pupil of approximately $6.5 \mu \mathrm{m}-7 \mu \mathrm{m}$ are terminated on opposite sites of the glass capillary and positioned such that both glass fibers are co-linear. Only when the glass fibers are well adjusted can cells be efficiently trapped in the capillary. If the output powers from the two fibers are identical, the trap is located in the center between the two 
fibers. However, detuning the laser power in one fiber results in a shift of the trap location along the beam axis. In this experiment, the output power in both fibers was identical.

The overall design of the microfluidic chip is sketched in Fig. 6.1(a). Note that, in contrast to an optical tweezer system, both laser beams are divergent which reduces radiation damage from the IR laser to a neglible minimum. The setup is also well suited to stretch cells. This can be achieved by significantly increasing the laser power up to approximately $1 \mathrm{~W}$ per fiber. The stretching of cells is due to an anisotropic stress profile on the cell contour as sketched in Fig. 6.1(b). This feature has not been used in the described experiment, but will certainly be exploited in future studies. Lastly, one should note that the center of the laser trap, defined by the positioning and alignment of the optical fibers during the assembly of the device, is not coinciding with the center of the glass capillary. Given a parabolic centro-symmetric flow profile in the capillary and a stable off-axis trapping center, a cell that is fixed in the trap will therefore experience a torque, as outlined in Fig. 6.1(c). The torque is directly proportional to the applied pressure and hence can be well adjusted by controlling the flow of the surrounding medium. The possibility of tuning the orientation of the cell in the trap can be beneficially used in, for example, tomographic experiments, as will be described later.

A combined system composed of a height leveling system and an air pressure pump was used to precisely vary the flow inside the glass capillary. The height leveling system is maintaining a $10 \mathrm{~cm}$ height offset between the cell suspension reservoir (inlet, placed $10 \mathrm{~cm}$ below the outlet) and the outlet of the fluidic channel. The air pressure pump is connected to the cell suspension reservoir and compensates the 10 mbar hydrostatic pressure. It can then be controlled within a range of $0 \mathrm{mbar}$ to 30 mbar with an accuracy of 0.1 mbar.

Before loading a new cell suspension, a syringe (1ml BD Plastipak with Luer-Lock, centric; Becton Dickinson GmbH, Germany) was used to inject an ethanol solution (95 wt\% ethanol in ultrapure water) into the microfluidic channel for disinfection and removal of residual trapped air bubbles. Then, the system was flushed with phosphate-buffered saline (PBS) and the cell suspension was injected. Lastly, the syringe was removed from the inlet tube and the tube was inserted into a vial filled with cell suspension. A magnetic stir bar was added to prevent sedimentation of the cells in suspension. The vial was then tightly sealed and the pressure pump was connected to an inlet of the vial.

The optical stretcher system is similar in terms of its operation principle to an optical tweezer. However, at this point one should point out that an optical stretcher 
offers several technical advantages compared with an optical tweezer. (i) Optical tweezers work at significantly higher flux density for cell trapping. Given an optical tweezer operating at laser powers $P_{\mathrm{OT}}$ from $50 \mathrm{~mW}$ to $200 \mathrm{~mW}$ and with a moderate beam radius $\omega_{0}$ of around $1 \mu \mathrm{m}$, the intensity in the laser focus can be estimated from $I_{\mathrm{OT}}=P_{\mathrm{OT}}\left(\pi \omega_{0}^{2}\right)^{-1}$. This yields an intensity ranging between $1500 \mathrm{kWcm}^{-2}$ and $6000 \mathrm{kWcm}^{-2}$. The optical stretcher described here was operated at approximately $20 \mathrm{kWcm}^{-2}$, approximated by $I_{\mathrm{OS}}=2 P_{\mathrm{OS}}\left\{\pi\left[d \tan \left(2 \lambda / a_{0} \pi\right)\right]^{2}\right\}^{-1}$, where $P_{\mathrm{OS}}$ was set to $100 \mathrm{~mW}$ per fiber, the distance $d$ between the exit pinhole and the trapping center was $80 \mu \mathrm{m}$ and the mode field diameter $a_{0}$ was $3.1 \mu \mathrm{m}$. (ii) Optical stretchers are well suited to trap large particles such as biological cells since the gradient of the electric field extends over the area of an entire cell, while in an optical tweezer the gradient of the electric field is typically only felt by a small portion of a cell. (iii) The elastic properties of cells can be measured by the stretching capability of the optical stretcher.

\subsubsection{Integration into the experimental endstation}

Data were recorded at the PETRA III endstation P10 (DESY, Germany). For the experiment, the sample chamber was integrated into the GINIX setup [63] which provides the opportunity for, both, X-ray nano-diffraction and propagation-based $\mathrm{X}$-ray imaging experiments [148]. The X-ray energy was set to $13.8 \mathrm{keV}$ using a $\mathrm{Si}(111)$ channel-cut monochromator to achieve a good compromise between phase contrast and a reasonable transmission through a significant amount of glass and liquid.

Due to the compact nature of the optical stretcher, the sample chamber was easily mounted on a motorized sample stage that consists of stepper motors (PI miCos GmbH, Germany) and a piezoelectric stage (SmarAct, Germany) for accurate positioning of the chamber, as depicted in Fig. 6.2(a). The microfluidic chip can be viewed in more detail in Fig. 6.2(b). In this setup, the sample can be viewed with two video cameras that can be inserted before and after the chamber. Both cameras can be used during X-ray exposures, since the beam is transmitted through a hole drilled into the mirror that projects the optical image on the respective CCD. For phase-contrast imaging experiments (see Fig. 6.2c) we have used KirkpatrickBaez (KB) focusing mirrors as a prefocusing device. The beam is hereby steered into the entrance pupil of a one-dimensional X-ray waveguide (wg) for coherence filtering. The optical stretcher is positioned along the propagation axis such that the capillary center is placed $20 \mathrm{~mm}$ into the defocus and the hologram is recorded 


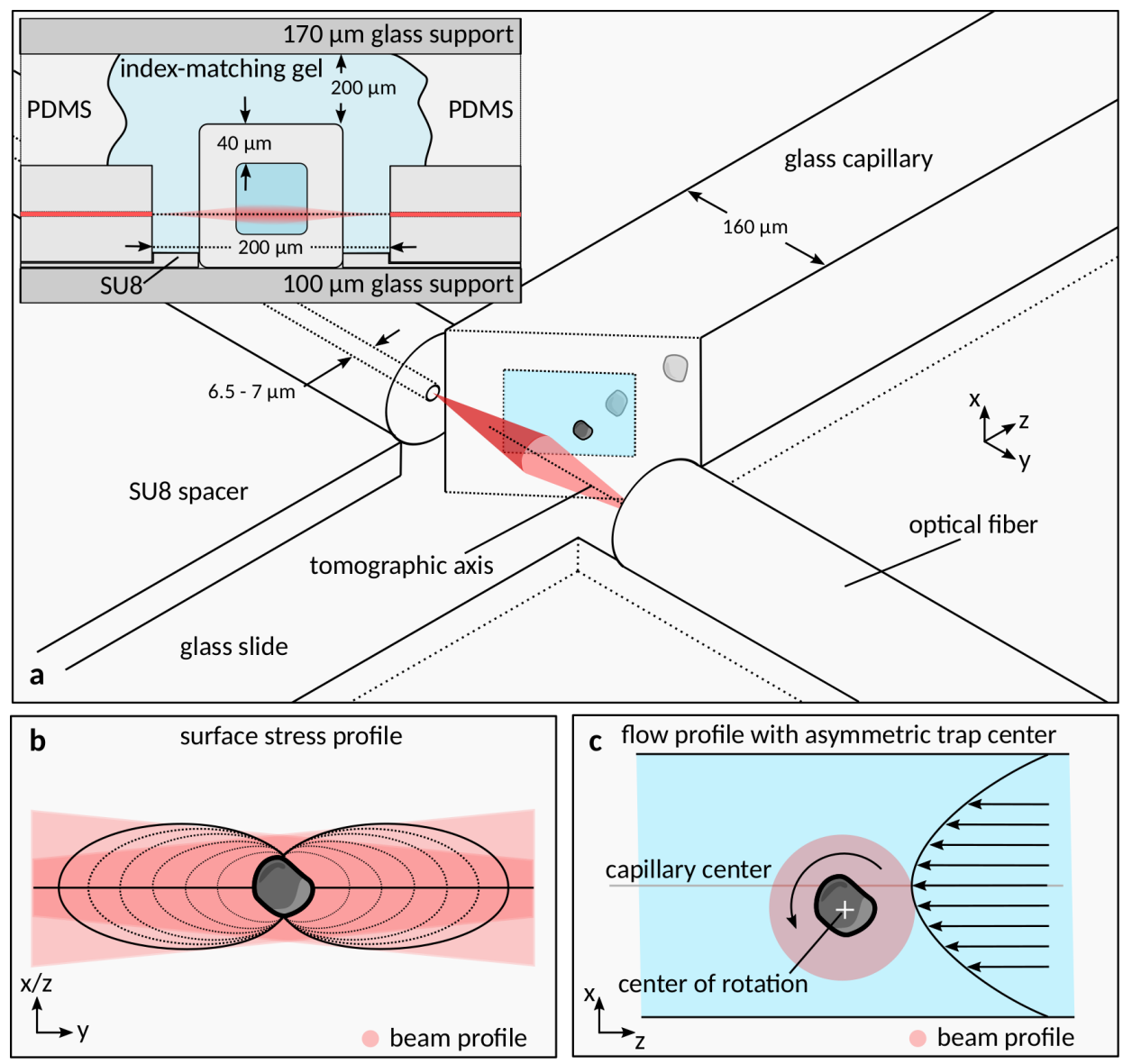

Figure 6.1.: (a) Sketch of the integration of the laser system on the microfluidic chip. The capillary is half-cut to give an inside view. Note that the laser beam axis also defines the tomographic axis. The inset shows the central $x-y$ cross section of the system. Along the propagation direction of the X-ray beam $(x)$, the beam transmits through a stack of materials: a supporting glass slide, the capillary glass and the containing fluid, a small layer of index-matching gel for optical microscopy and a covering glass cover slip. (b) Highly anisotropic stress profile on the cell contour when a cell is trapped or stretched (adapted from [229]). (c) Application of a torque on a trapped cell due to the off-axis positioning of the laser trap. 


\section{0 \\ The optical stretcher as a tool for single-particle X-ray imaging and diffraction}
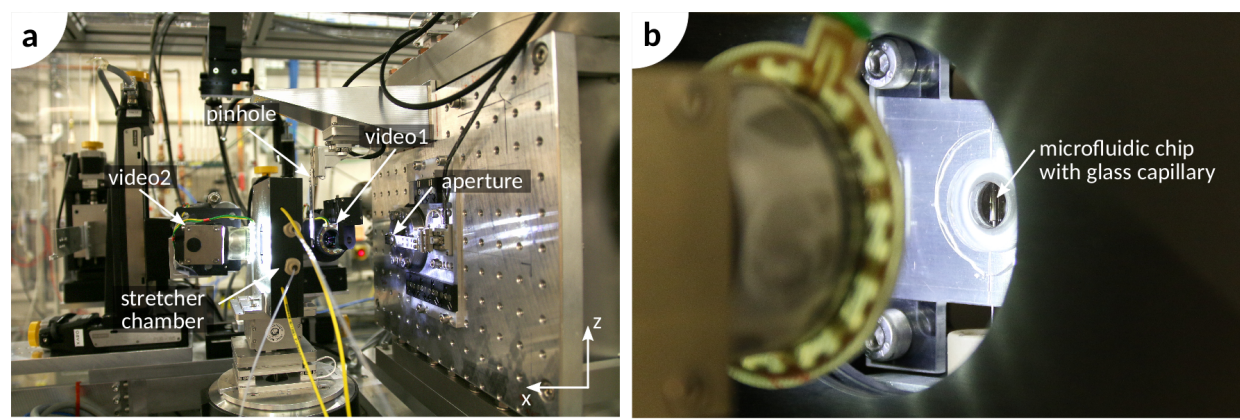

C

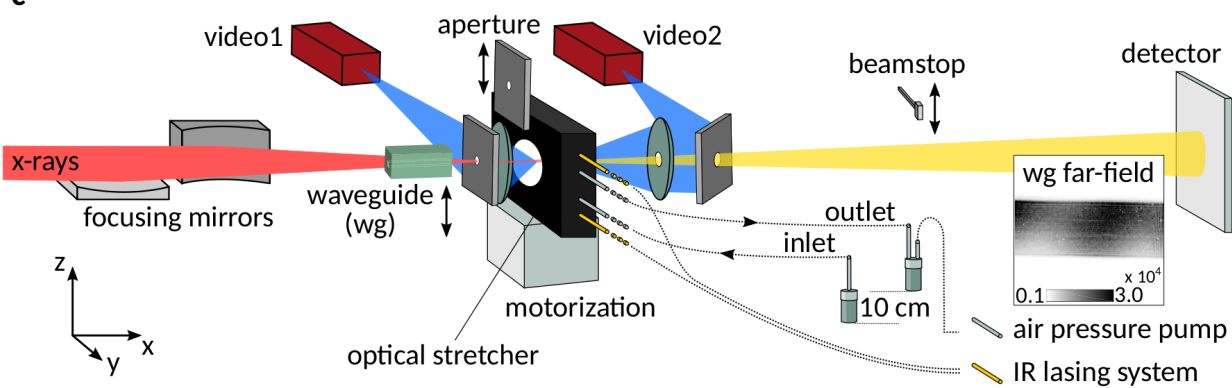

Figure 6.2.: The optical stretcher mounted into the GINIX setup of the P10 endstation at PETRA III, DESY. (a) Photograph of the setup during alignment with the X-ray beam. (b) Close-up photograph of the interior of the chamber. Upon close inspection, one can observe the $160 \mu \mathrm{m}$ thick vertical capillary and the index-matching gel. (c) Experimental configuration for $X$-ray phase-contrast imaging and scanning $X$-ray diffraction. In the phase-contrast setting, $K B$ focusing mirrors steer the beam into the entrance pupil of a one-dimensional $X$-ray waveguide (WG). The far-field pattern of the $X$-ray beam is shown in the inset. For scanning diffraction studies, the stretcher chamber is placed into the focus of the KB beam. A beamstop is inserted to block the primary beam. Note that in both configurations the stretcher chamber is connected to a height leveling system and an air pressure pump for flow control as well as an IR lasing system for optical trapping. For a detailed description of all components depicted in (c), see Sec. 6.2.2.

on a scientific CMOS-based detector (pixel size $\Delta x=6.5 \mu \mathrm{m}$; Photonic Science, UK) placed $5.04 \mathrm{~m}$ downstream of the focus position. In this configuration, the magnification $M$ is dependent on the position of the sample $z_{01}$ and the detector $z_{02}$ with respect to the focus position of the beam. The magnification is therefore $M=$ $z_{02} / z_{01}=5.046 \mathrm{~m} / 0.02 \mathrm{~m}=252.3$ and the effective pixel size is $\Delta x_{\text {eff }}=\Delta x / M=$ $6.54 \mu \mathrm{m} / 252.3=25.9 \mathrm{~nm}$. The total photon flux is $I_{0}=7.7 \times 10^{9}$ photons $^{-1}$.

For initial alignment of the chamber, we have removed the waveguide and placed the capillary directly into the focus of the KB beam. In this setting [see Fig. 6.2(c), waveguide moved out of the beam, aperture and beamstop are aligned in the beam], 

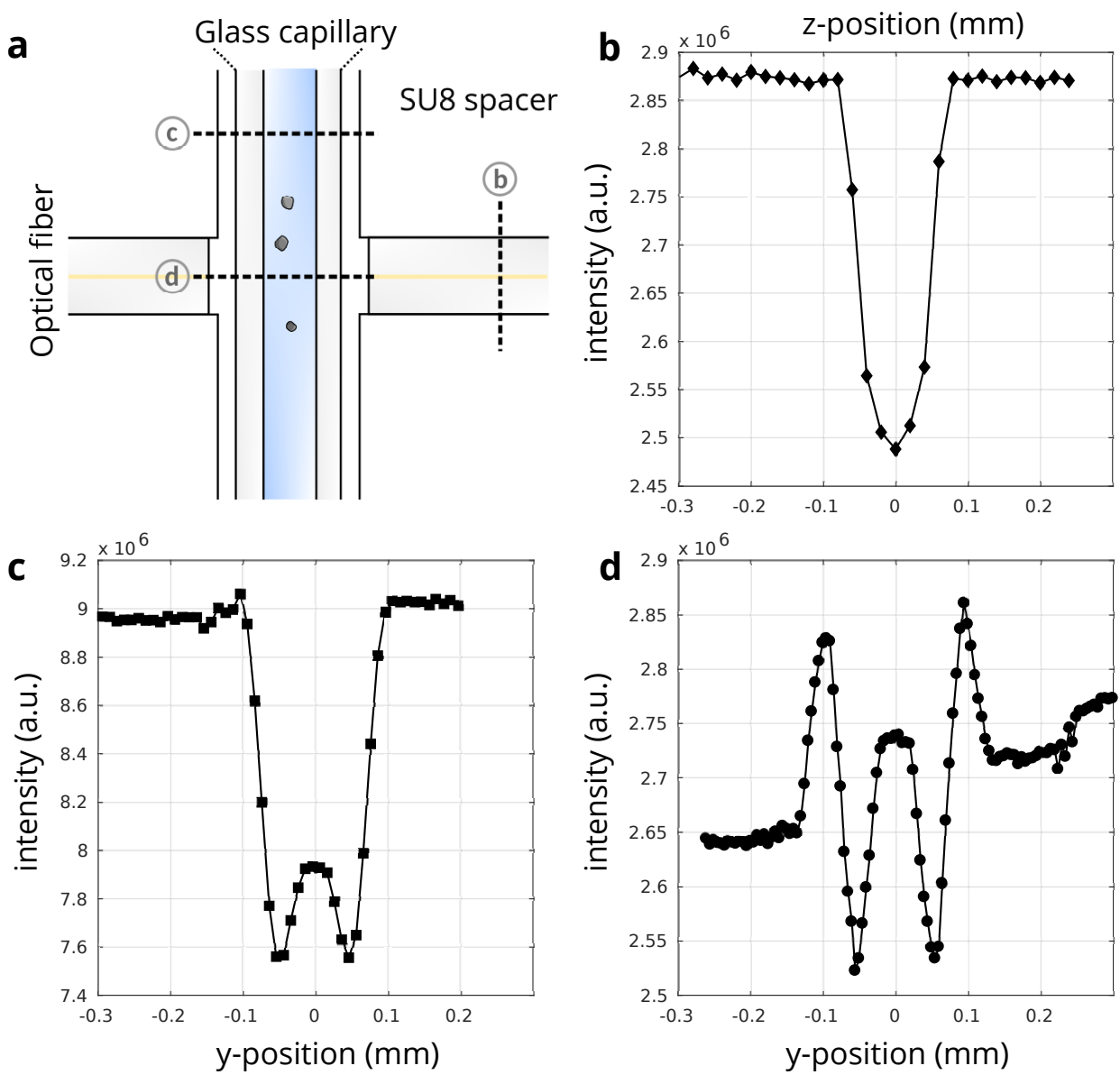

Figure 6.3.: Absorption profiles through the optical fiber, glass capillary and the trap region. (a) Sketch of the crossing region between the glass capillary and laser fibers. Black dashed lines are enumerated and correspond to the absorption line profiles in (b), (c) and (d).

typically used for microfocus and nanofocus diffraction experiments, the focus of the beam is approximately $307 \mathrm{~nm} \times 295 \mathrm{~nm}$ in size and the overall photon flux is $I_{0}=9.4 \times 10^{10}$ photons $^{-1}$. A movable aperture can be inserted to improve the beam profile several millimeters in front of the sample. To find the intersection between the optical fibers and the glass capillary (see Fig.6.3a), we have scanned the KB beam vertically along one optical fiber (Fig. 6.3b) and horizontally along the glass capillary (Fig. 6.3c). The scan along the intersection point is shown in Fig. 6.3(d). 

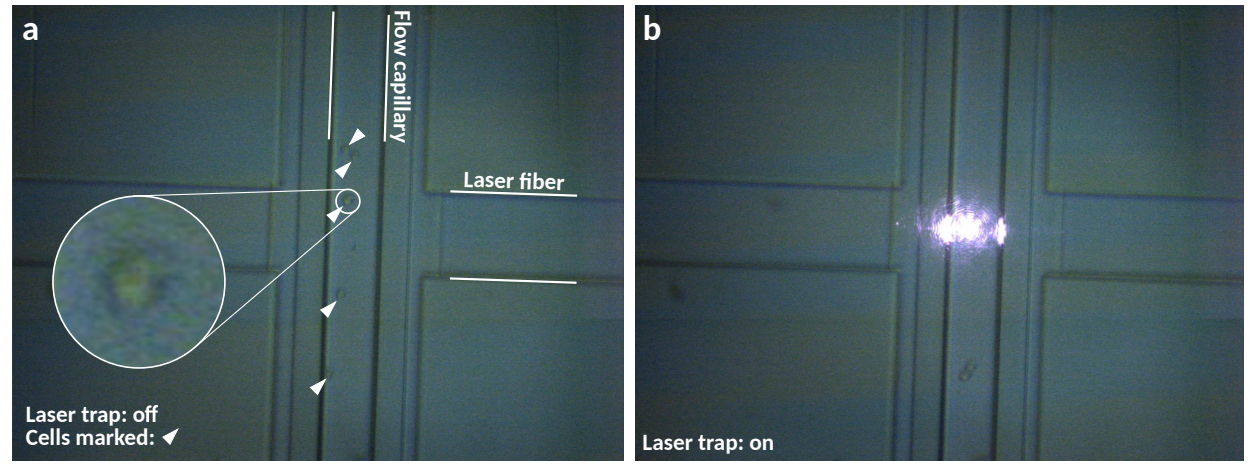

Figure 6.4.: Micrographs of the trapping region obtained using trans-illumination. In (a), chemically fixed macrophages are inserted into the microfluidic channel. Single cells are clearly visible in this imaging mode, as can be seen from the inset. (b) Upon laser trapping, the cell is pulled into the trap center. Light scattering from the walls of the capillary and from the trapped cell is clearly visible. Here, a trapping power of $100 \mathrm{~mW}$ was used.

Following this initial alignment, we have tested the optical trapping of cells in the capillary. For this, we have used chemically fixed macrophages (MH-S, ATCC CRL-2019; ATCC, USA) with a diameter typically ranging between $10 \mu \mathrm{m}$ and $25 \mu \mathrm{m}$. The cells could be clearly identified in the capillary as shown in Fig. 6.4(a). Once the trap (here, $100 \mathrm{~mW}$ output power per fiber) is activated and a cell is trapped, it is moved into the center of the laser trap. In the optical micrograph, successful trapping can be easily seen by strong scattering of the IR radiation from the cell. Due to photon conversion in the CCD chip, this is clearly visible by a bright violet glow, as depicted in Fig. 6.4(b).

\subsection{Results}

\subsubsection{Polystyrene beads}

The following experiments have been conducted in the holographic setting as described in Sec. 6.2.2 and depicted in Fig. 6.2(c). As an initial test sample that provides adequate contrast relative to the surrounding medium, we used polymer beads (4210A; Thermo Scientific) with a diameter of $10 \pm 0.08 \mu \mathrm{m}$ and a density of $\rho_{\mathrm{PS}}=1.05 \mathrm{~g} \mathrm{~cm}^{-3}$ in a solution containing $90 \mathrm{vol} \%$ ethanol and $10 \mathrm{vol} \%$ ultra-pure 
water. The maximum phase shift that can be expected is in this case

$$
\Delta \varphi=\varphi_{\mathrm{PS}}-0.9 \varphi_{\text {ethanol }}-0.1 \varphi_{\text {water }}=-0.084 \mathrm{rad}
$$

For reconstruction of the electron density we have used a phase-retrieval method based on the contrast transfer function (CTF) [100] which is a single-step procedure as well as an iterative method (using the support of the cell as well as negativity of the phase as constraints) based on the RAAR algorithm (RAAR: relaxed averaged alternating reflections) [234]. In Fig. 6.5(a) the original hologram recorded with an exposure time of $0.5 \mathrm{~s}$ is shown after division with an empty profile. A black line indicates the central line around which ten lines have been averaged to yield the horizontal line profile shown on the right.

The result of CTF reconstruction is shown in Fig. 6.5(b). Residual low-frequency components could not be removed without further deterioration of the reconstruction quality. Also, horizontal line profiles resulting from horizontal streaks from the KB illumination were amplified in the reconstruction. In contrast to the singlestep reconstruction, these artifacts could be minimized using the iterative RAAR algorithm, as shown in Fig. 6.5(c). The RAAR reconstruction was run for 500 iterations. A circle with a radius of $10.6 \mu \mathrm{m}$ was chosen as support and is indicated by a dashed red line. The corresponding line profile does not yet fit the expected half-circle shape. This may be due to the poor recovery of the small spatial frequencies during the holographic reconstruction. Despite this, the corresponding line profiles shown on the right for each image (a)-(c) confirm, that the RAAR reconstruction performs well in reproducing the phase in the interior of the bead. Taking a movie series of a single polymer bead we observed that during an observation period of approximately $10 \mathrm{~s}$ the polymer bead remained accurately trapped with a positional accuracy of $\operatorname{Var}(y)=310 \mathrm{~nm}$ and $\operatorname{Var}(z)=404 \mathrm{~nm}$. The position of the particle was obtained by calculating the center of mass of a thresholded binary image of the reconstructed phase. Based on the equipartition theorem, the variance of the particle movement would translate into a trap stiffness of $k_{x}=k_{B} T \operatorname{Var}(x)^{-1}=1.0 \times 10^{-3} \mathrm{pN} \mathrm{nm}^{-1}$ and $k_{y}=k_{B} T \operatorname{Var}(y)^{-1}=$ $0.8 \times 10^{-3} \mathrm{pN} \mathrm{nm}^{-1}$, given an ambient temperature of $23^{\circ} \mathrm{C}$. In addition to a single trapping location, further side minima of the trapping potential along the beam axis might lead to 'hopping' of a trapped particle. The trap stiffnesses reported here are based on a rough estimation of the particle movement. Future applications of the technique will require a more complete characterization of the trap stiffness taking into account, for example, different trapping powers, particle diameters and 

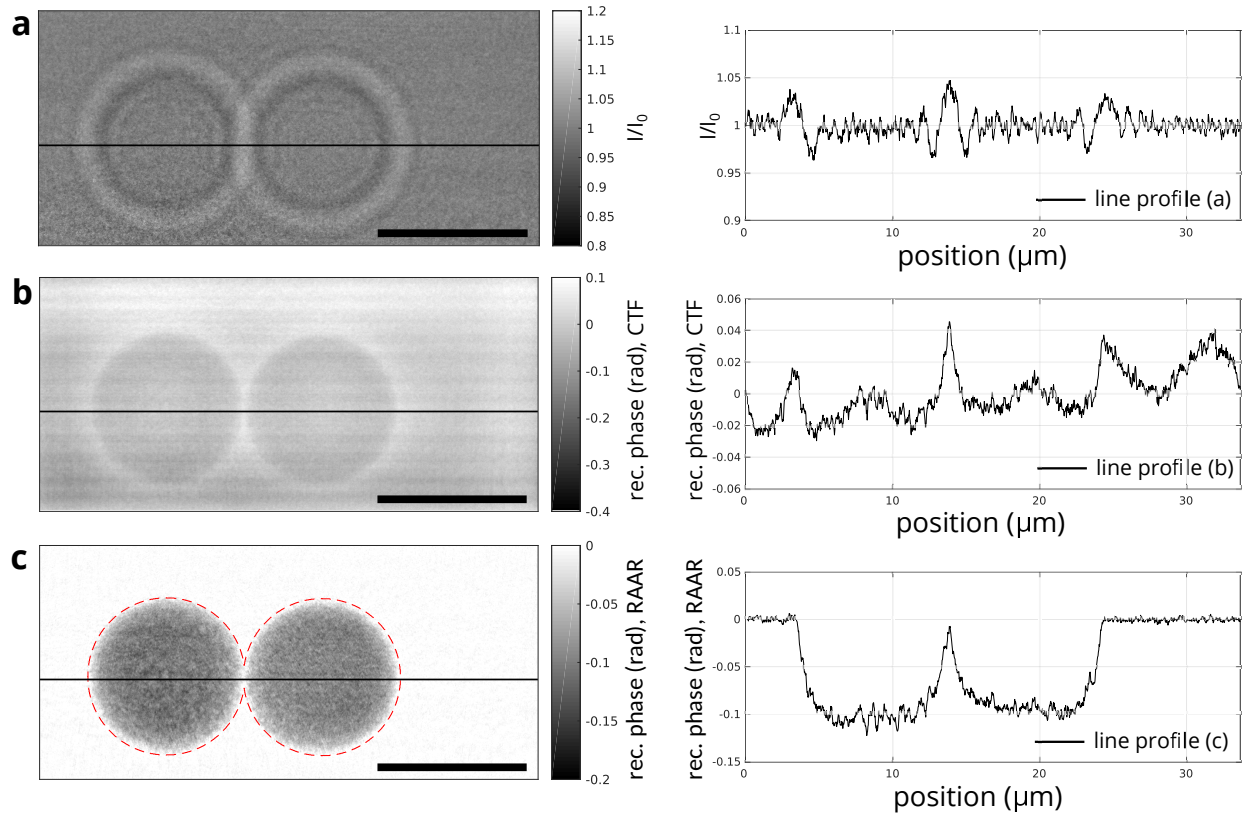

Figure 6.5.: Comparison of reconstruction results from holographic projections of latex beads. (a) Hologram of two latex beads in a laser trap. (b) Direct reconstruction based on the contrast transfer function. (c) RAAR-based iterative reconstruction after 500 iterations. Support is indicated by a red dashed line. Line profiles from the data shown in (a-c) are in each case shown on the right. The line profile was calculated by averaging ten lines around the central line. The position of the central line is indicated on the left by a solid black line. Laser trapping power: $100 \mathrm{~mW}$. Scale bar: $10 \mu \mathrm{m}$.

longer recordings of the particle movement.

\subsubsection{Macrophages loaded with barium sulfate}

As a next step, we recorded holograms from chemically fixed macrophages. Since the contrast between suspended single cells and the surrounding medium is very low, we additionally stained the macrophages with barium sulfate, a common contrast agent in computed tomography.

To label the cell culture with barium, we have added $10 \mu \mathrm{l}$ barium sulfate solution (Micropaque CT, Guerbet, Germany) per $2 \mathrm{ml}$ cell medium. The cell suspension was gently mixed with barium sulfate using a pipette. The solution was then incubated for $4 \mathrm{~h}$ at $37^{\circ} \mathrm{C}$ and $5 \% \mathrm{CO}_{2}$. After incubation, the cells were gently scratched off the surface of the petri dish and centrifuged at 700 r.p.m. for 7 min. The supernatant was then removed and the cells were chemically fixed in a formalin 
solution (9 wt\% formalin in PBS). After a fixation period of several hours, the cells were washed three times in PBS before being used in an experiment.

Barium-labeled macrophage solution was added to the optical stretcher and a single cell was trapped using a trapping power of $100 \mathrm{~mW}$ per fiber. A small pressure was applied to the capillary inlet, such that the cell remained trapped but rotated in the capillary. During one rotation, 14 projections were acquired and divided by an empty projection as shown in Fig.6.6(a). The exposure time for each projection was $0.1 \mathrm{~s}$ and the dose per projection shown in Fig. 6.6 was 927 Gy. Since the dead-time of the detector after each exposure was $1.1 \mathrm{~s}$, an exposure time of $0.1 \mathrm{~s}$ corresponds to a cell rotation of $2.1^{\circ}$ per projection and one full rotation of the cell took $16.8 \mathrm{~s}$. Each projection was then iteratively reconstructed using 500 iterations of the RAAR algorithm. The reconstructed phase is shown in Fig. 6.6(b). The support has been manually chosen and is indicated as a red dashed line.

Barium sulfate is clearly resolved in the reconstruction. Even the smallest grains of size smaller than $500 \mathrm{~nm}$ are clearly visible. The cell contour on the other hand is more difficult to discriminate against the background. Darker regions in the reconstruction seem to depict the cytosol while the contour of the cell is not traceable. It appears that the reconstruction can still recover the cell cytosol even though it is not visible by eye in the projection data.

\subsubsection{Three-dimensional iterative reconstruction of a rotating macrophage}

Based on the data shown in Fig. 6.6, it should in principle be possible to retrieve the phase of the object in three dimensions. Due to a very low number of projections, we have chosen to reconstruct the three-dimensional phase by employing the simultaneous iterative reconstruction technique (SIRT) [235]. We have used the algorithm as implemented in the ASTRA toolbox [236, 237].

For an optimal reconstruction, the data had to be preprocessed prior to the reconstruction. First, to estimate the drift of the cell along the tomographic axis (y-axis), the reconstructed phase in Fig.6.7(a) was thresholded to yield a binary image as shown in Fig. 6.7(b). The center of mass of the three largest connected components of the binary image were calculated and the procedure was repeated for all 14 reconstructions shown in Fig. 6.6(b). This procedure yields the $y$ - and $z$-position for each reconstructed frame. The shift along the $y$-axis (Fig. 6.7c) was used to register each frame such that the cell remains horizontally fixed. The $z$-axis of each grain is shown in Fig. 6.7(d) and confirms that one period of rotation is 

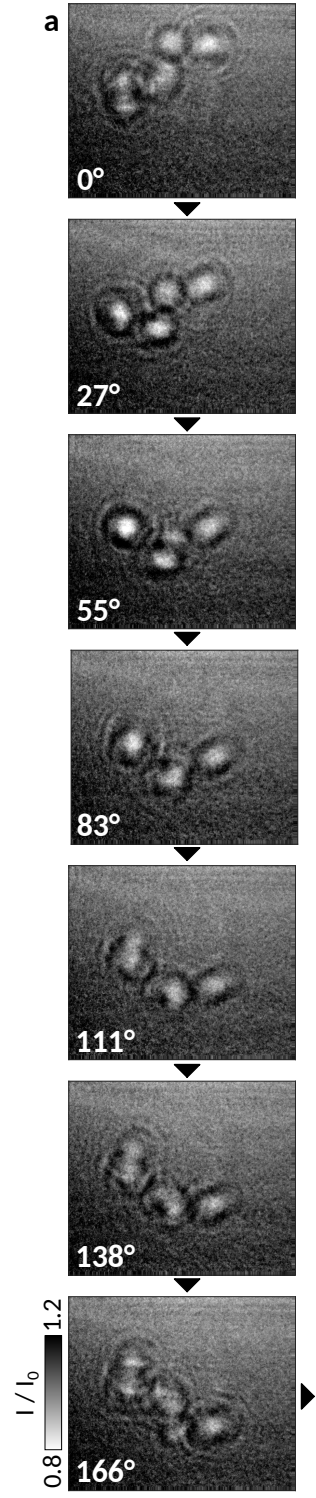
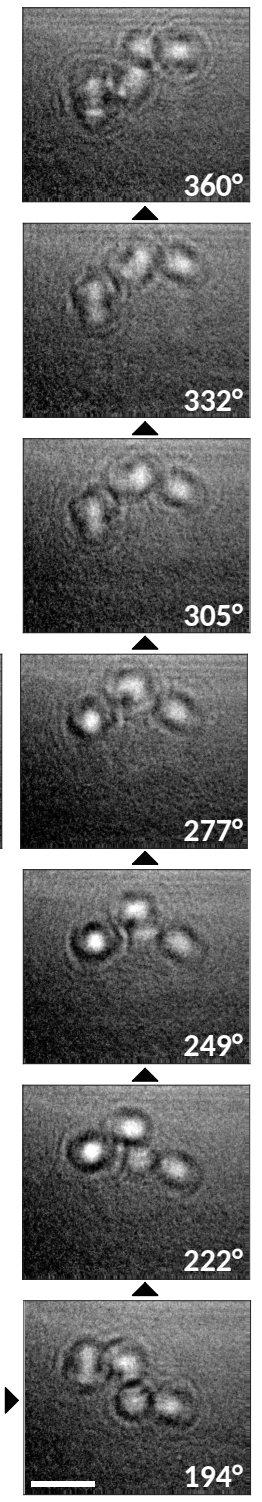
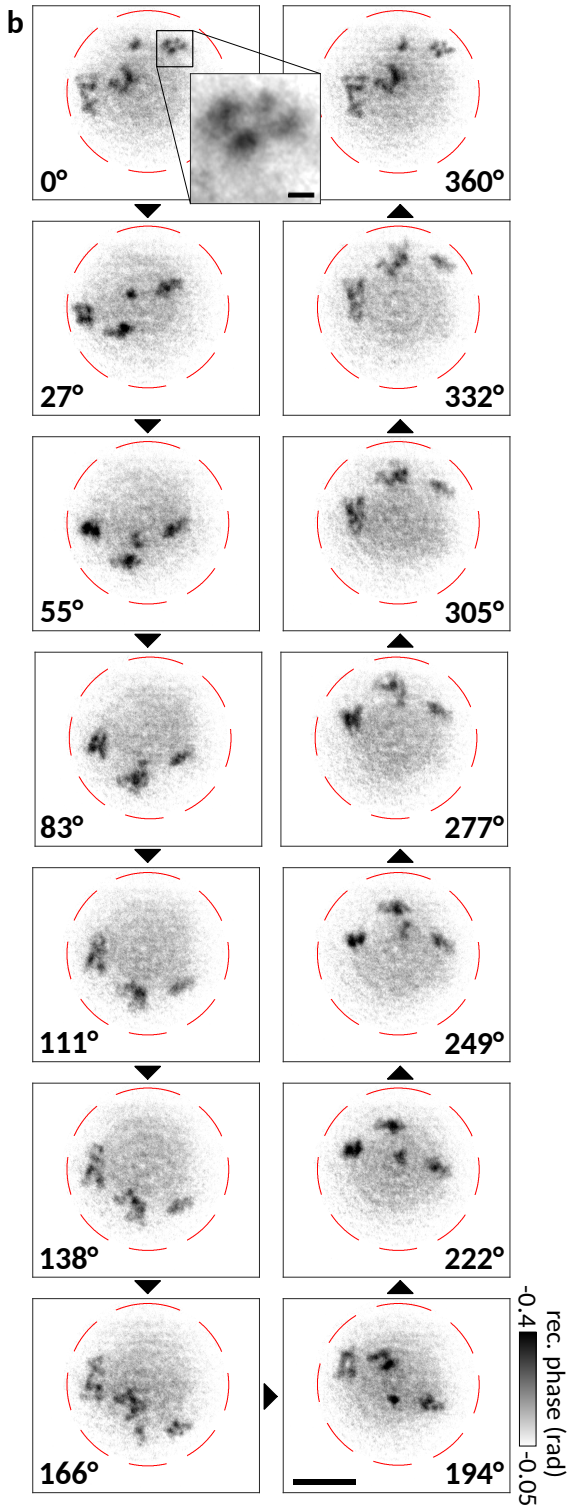

Figure 6.6.: Holographic projections and iterative reconstructions from barium-stained macrophage during rotation in an optical trap. (a) Holographic recordings of the rotating cell. Strong fringes appear due to the barium sulfate stain. (b) Barium sulfate grains become clearly resolved upon iterative reconstruction. Here, 500 iterations using the $R A A R$ scheme have been used for a good reconstruction. Higher gray values in the center of the cell seem to depict the cell body. The inset shows single barium sulfate particles. Laser trapping power: $100 \mathrm{~mW}$. Scale bar: $5 \mu \mathrm{m}$. Scale bar of inset: $500 \mathrm{~nm}$. 
sampled. Secondly, after registering the cell position, the reconstructed data were binned $(4 \times 4$ binning $)$ to reduce artifacts stemming from noise in the reconstruction. Lastly, the set of projections was reconstructed in a parallel beam geometry using the ASTRA toolbox and an effective pixel size of $103.7 \mathrm{~nm}$.

To illustrate the final reconstruction result, three views on a volume rendering are shown in Fig. 6.7(e). The volume rendering effect was generated using the Avizo $3 \mathrm{D}$ visualization software (Avizo Lite 9.2.0, FEI, USA). In orange, the cytosol of the cell is clearly visible. Single barium sulfate grains as seen in the single reconstructions were not retrieved but the barium sulfate aggregations are clearly reproduced and shown in blue. For clarity, markers are added to identify identical barium grains. Due to the limited amount of angles, streak-like artifacts are still visible in the reconstruction.

\subsection{Summary and conclusion}

As we have shown, the dual-beam optical trap and stretcher is a promising tool for X-ray imaging of biological cells in the hydrated state by synchrotron radiation and FEL. The holographic reconstructions here were severely limited by the small signal, which in turn required long exposure times. Given the fact that the particles undergo slight movements, the holographic fringes wash out and the small contrast is further diminished. Single FEL exposures unaffected by any motional blurring would therefore provide substantially higher signal, and presumably electron density maps of much higher resolution. At the same time, the background signal of the yet non-optimized microfluidic chip is still substantial and would need to be reduced. With less material to be penetrated, the photon energy could also be lowered which would in turn lead to a much stronger contrast.

We also pointed out that, in single-cell experiments, the optical stretcher offers critical advantages compared with an optical tweezer. One key advantage is that, due to the use of a dual beam optical trap with divergent laser beams, cells can be deformed using significantly higher laser powers without damaging or even opticution of the cell. This brings about the possibility to probe structural changes as a function of the stretching force. By such means, one could, for example, probe stress-induced structural variations of the actin cortex of cells. Joint investigations of structure by X-ray diffraction, and of elasticity using the stretching capability, would also be extremely interesting for studies of vesicles.

The use of a dual-beam optical trap offers the second advantage, that larger par- 


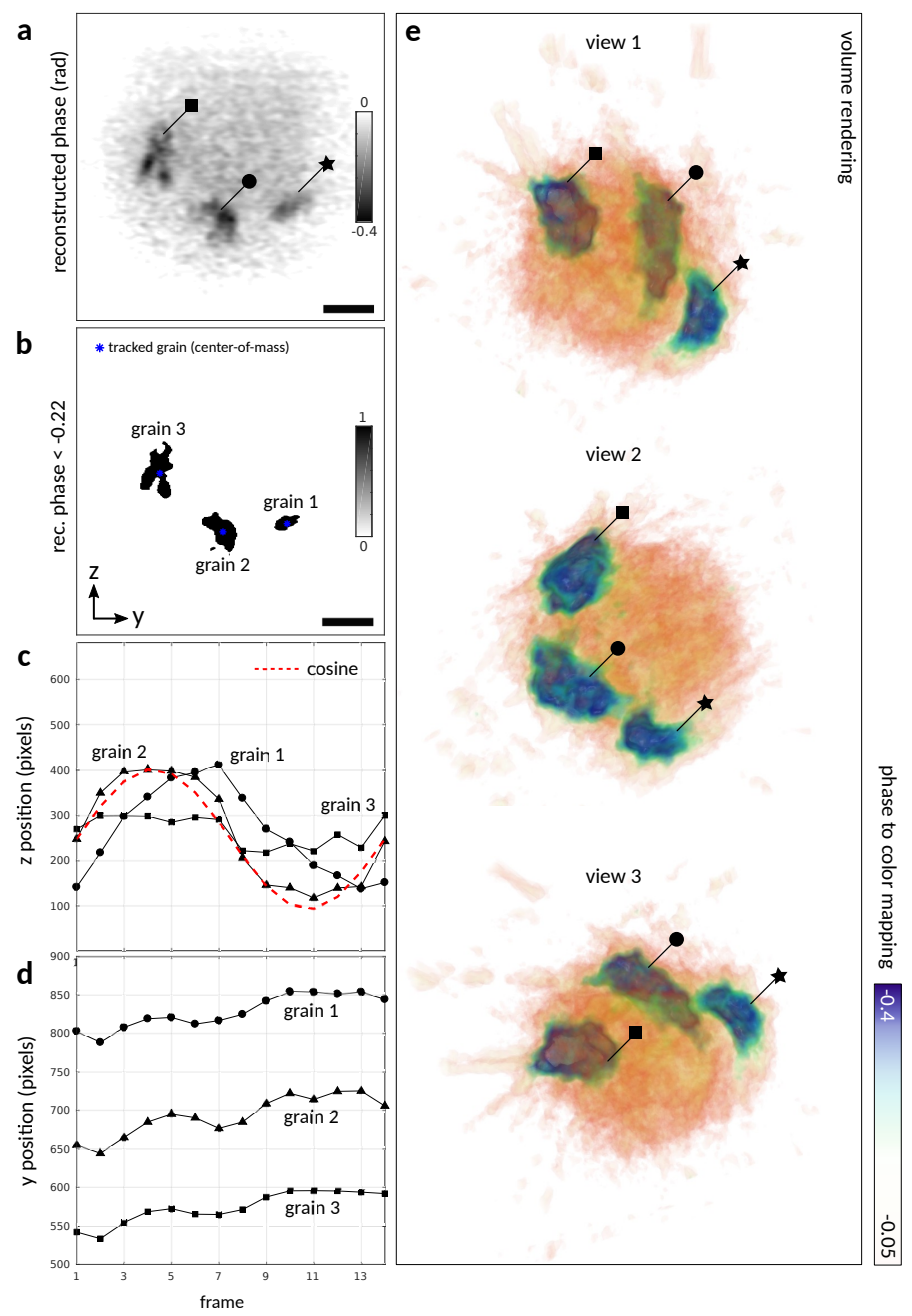

Figure 6.7.: Reconstruction of the barium grains in a three-dimensional volume. (a) Iterative reconstruction (low-pass filtered) of a single frame as shown in Fig. 6.6. (b) Binary image using a threshold of $-0.22 \mathrm{rad}$. Black regions indicate phases smaller than the threshold value. The three largest connected components in the binary image are tracked in all frames. (c,d) Tracked $y$ - and z- position of the barium grains throughout the scan. Note that all frames are shown in Fig. 6.6 and used for iterative reconstruction of the three-dimensional volume. (e) Volume rendering of the reconstructed $3 D$ phase. Reconstruction was achieved using 50 iterations of the SIRT algorithm implemented in the ASTRA toolbox. Prior to reconstruction, the data had been corrected for shifts along the $y$-axis and the rotation axis was placed manually into the center of rotation. Markers are used to indicate identical barium grains. Scale bar: $2.5 \mu \mathrm{m}$. 
ticles, in particular suspended cells, can be more easily trapped. We have furthermore shown that, in combination with an X-ray probe, flow control can be very effectively used as a micromanipulation technique that even enables tomographic imaging of cells. This manipulation method, however, has a very low position accuracy and does not work well for particles with an irregular surface. In future experiments, the trapping functionality will therefore be modified such that the sample can be micromanipulated more precisely by optical means.

For all these applications, high throughput and automated analysis is indispensable for conclusive and relevant results, given the polydispersity and polymorphism of the objects studied. While automation relies on the detection of objects in the microfluidic channel, as we saw here, even the modest beamline optical microscope would already provide the required optical control. To this end, it seems that the dual-beam optical trap and microfluidic sample conditioning and delivery is an ideal tool, given the high throughput already demonstrated in its conventional operation without an X-ray probe.

\section{Appendix}

\section{A1. Rotation of a macrophage}

Movie S1 of the supporting information contains a short movie sequence showing the trapping and rotation of a macrophage as viewed in the standard commercial phase-contrast setup. The pump pressure was adjusted such that the cell did not move inside the trap, then the pressure was increased by approximately $100 \mu$ bar and the cell started rotating smoothly around its fixation point in the optical trap. Note that the rotation velocity as well as the precise rotation axis is dependent on the shape of the cells. The maximum rotation speed of the cell also critically depends on the trap stiffness. Here, the cell was performing one revolution in approximately $16 \mathrm{~s}$.

\section{A2. Tracking of macrophages in the microfluidic channel}

In movie S2 of the supporting information the outline of the cell was tracked while it was flowing through the capillary channel. In (a) the raw data aquired with the on-axis beamline microscope are shown, while in (b) each raw frame was empty subtracted and smoothed. To track the cell, the pre-processed image was thresholded and the center of mass of each connected component in the binary 
image was tracked (here, a single component is shown). The tracked cell is outlined with a red circle. This demonstrates that the limited capability of the on-axis microscope is sufficient to detect cells in the channel, hence enabling an automatic mode that traps, images and releases cells automatically in a high-throughput fashion.

\section{A3. Trapping of a macrophage}

Movie S3 of the supporting information shows an unstained macrophage that is trapped and released multiple times during the clip duration.

All videos are available as avi files using Motion JPEG encoding and can be viewed, for example, using the VideoLAN VLC software (https://www.videolan. org/vlc/).

\section{Acknowledgements}

We thank Markus Osterhoff for continuous developments at GINIX, Bastian Hartmann for engineering support, and Malte Vassholz for support in the alignment of the experimental setup. We are also grateful for the competent advice by the DESY laser safety team prior, during and after the experiment. We thank RS Zelltechnik and in particular Roland Stange for providing the instrument and for excellent support as well as helpful discussions.

\section{Funding information}

Funding for this research was provided by: Deutsche Forschungsgemeinschaft (grant No. SFB 937, project A11); Bundesministerium für Bildung und Forschung (grant No. O5K16M62). 


\section{Summary and outlook}

This thesis has implemented scanning X-ray diffraction and near-field holographic imaging for the imaging of cardiac cells and tissues. For this purpose, sample preparation protocols, experimental parameters and setups as well as data analysis routines have been evaluated and were optimized. In particular, a data analysis framework has been developed to automatize and standardize the analysis routines and process large amounts of data at high throughput, as described in Chapter 3. Importantly, this progress was motivated by advances in the recording of scanning diffraction data, most importantly the implementation of a continuous scanning scheme and fast, synchronized recordings with pixel detectors and data storage.

Even with the right tools at hand, the scattering signals from neonatal cells were found to be surprisingly difficult to interpret and due to the absence of any typical muscle peak, the data was interpreted heuristically. This situation has changed when imaging adult cardiomyocytes and cardiac tissue. In this case, the characteristic equatorial reflections have been observed and could be mapped with unprecedented resolution in real space. The cause for the discrepancy between the scattering signal from neo-natal and adult cells is still elusive since the diffraction signal strongly dependents on (i) the signal-to-noise ratio, (ii) the radiation sensitivity of the sample and (iii) structural changes due to the sample preparation.

To disentangle these separate effects, we have studied different sample preparation methods, as presented in Chapter 4 . While adult cardiomyocytes and adult tissue could be chemically fixed and imaged in solution without further processing, neonatal cells were rendered radiation resistant by freeze-drying or cryogenic fixation and in addition have also been studied in the living state. The absence of the muscle diffraction signal in neo-natal cells for all preparation states was surprising in view of the observed contractile function in these cells. We must conclude that signal levels in these experiments were simply too low. Whether a larger beam size will be sufficient to accumulate more signal remains to be seen. In any case, we can advise that nanofocused X-rays should only be used for projects where high real space resolution is indispensable. In this case, radiation damage must be mitigated by cryogenic fixation. Furthermore, in adult cardiomyocytes from mice, we could observe significant changes due to the chemical fixation protocol and 
mouse treatment. Based on this technical progress and proof of concept, it now remains to be studied in greater detail whether the lattice spacing could be used as a structural biomarker for clinical studies.

To this end, Chapter 5 gives an example how scanning X-ray diffraction can be used to study variations in the tissue ultrastructure and elemental composition due to Parkinson's disease. In this example we showed that iron is enriched and copper diminished in dopaminergic neurons, in line with the general understanding of the neuropathology of the disease. Interestingly, crystals have formed on the tissue sections which we could identify as aggregates of anhydrous cholesterol and localize by diffraction contrast. Along with X-ray fluorescence and crystalline diffraction, we also observed myelin signals by scanning small-angle X-ray diffraction. The myelin signal was surprisingly heterogeneous and at sparse locations the myelin sheath appeared to have undergone a phase transition to an hexatic phase. The underlying cause for this phase transition is yet unknown and will be subject to future studies.

Chapter 6 presented an approach to combine scanning X-ray diffraction with holographic imaging which offered several advantages. First and foremost, X-ray holography could increase the resolution range accessed in the diffraction experiment by two orders of magnitude with almost no gap between the two resolution ranges. Secondly, it could be used as a tool to observe radiation damage, although not simultaneously.

The combination of holographic imaging with scanning X-ray diffraction has proven useful because two different contrasts are exploited that are however based directly or indirectly (in form of correlations) on the electron density of the sample. Chapter 4 has extended this correlative approach by combining scanning X-ray diffraction with fluorescence microscopy. The specific labeling of a structure can in this case be used to understand the origin of the scattering signal.

The capabilities of scanning X-ray diffraction and holographic imaging can be expected to increase further with the availablity of fourth-generation synchrotrons as well as XFELs. Chapter 6 presented the development of an optical stretcher that could be used for scanning X-ray diffraction and holographic imaging on hydrated, suspended cells. Besides cells, other micrometer-sized particles could be studied, with applications ranging from the study of neurodegenerative diseases in model systems to the development of micrometer-sized containers for drug delivery. Although the current design of the optical stretcher would require adaptation, most certainly when used at XFELs, in its current state it already seems to be a promising tool for research at fourth generation synchrotrons and XFELs. The 
ability to deform cells is again an interesting link to the diffraction studies on cardiomyocytes as this could enable stretch-and-release type of experiments on the level of a single cardiomyocytes which would clearly be a revealing extension of diffraction experiments that are currently being performed on isolated muscle fibers.

Much of what has been presented here can be labeled as exploratory. However, based on the data presented in the previous chapters, interesting trends can already be identified and the application of scanning X-ray diffraction and holographic imaging for cardiac research in particular seems to be a path absolutely worth taking. 

Appendix 



\section{A. Experimental aspects of scanning X-ray diffraction}

The principle of scanning X-ray diffraction seems intriguing as it potentially offers similar information as a diffraction experiment with the added benefit of real space resolution. However, as for classical X-ray diffraction, scanning diffraction experiments require careful planning and adjustment of all experimental parameters influencing the signal level. Two key limitations that have been mentioned in this thesis are radiation damage and the overall experimental layout. Several strategies to circumvent radiation damage have been outlined here. Motivated by the findings presented in this work, it became clear that recordings of vitrified samples would be necessary in the future and that cryogenic protection in a flow of cold nitrogen gas is not sufficiently reliable for this purpose. Recording scanning diffraction data on $\mathrm{LN}_{2}$-cooled samples then requires the use of a vacuum chamber. The initial development of a cryogenic chamber for scanning applications is described in the following.

Furthermore, signal levels can be improved by minimizing spurious background scattering. For beamlines that operate in air, it is particularly important to minimize the distance the X-ray beam propagates in air. One way to remove air scattering from the intense X-ray beam downstream of the sample is by blocking the beam as early as possible without interfering with the scattered radiation. For scanning diffraction experiments at beamline P10, DESY we have developed a 3D printed device that contains a beamstop placed into a helium-flushed tube mounted directly behind the sample to remove downstream air scattering. The design is strongly influenced by an implementation at beamline ID13 of the ESRF developed by Britta Weinhausen and Manfred Burghammer.

Cryogenic environments for scanning applications In the sample environment development for X-ray applications, cryogenic systems have become of great interest, especially for highly dose-demanding applications such as X-ray diffraction microscopy [83, 238], ptychography [157], X-ray fluorescence [156], or ptychographic tomography [239]. It is still an open question how small a cryo- 
genic chamber can be manufactured to contain a fully motorized sample stage and a sample transfer system and still provide adequate thermal isolation. Thus far, cryogenic environments at synchrotron endstations are bulky developments with little modularity, e.g. the experimental setup at beamlines ID16A or ID21 of the ESRF or [155-157]. Beamlines operating in air would benefit from a smaller design that could be shared by multiple endstations. In fact, cryostats have been used for this purpose but their use is limited as they (i) do not usually contain a cryogenic sample transfer, (ii) are generally not compatible with the constraints on nano-diffraction beamlines such as short working distances of multilayer Laue lenses and nanofocusing lenses, and (iii) would require external scanning which can not be done with high resolution scanning stages (e.g. piezo-stages) due to load constraints. Regarding (iii), the necessity to scan the entire cryostat can be circumvented by scanning the focusing optic through the X-ray beam. This approach is however also flawed as the beam profile is usually not homogeneous throughout the scanning range.

Martin Rosenthal and I have therefore proposed to develop a cryogenic chamber with minimal dimensions that integrates a piezo scanner into the vacuum chamber. The cryogenic system is based on a vacuum chamber with very small dimensions compatible with the constraints of nano-diffraction setups that should allow for fast and accurate scanning of the sample. The sample will be mounted on a flexible element of a liquid nitrogen-cooled heat exchanger and will be scanned with the piezo scanner that is placed inside the cryostat chamber. The chamber is constructed to be highly modular in design to allow for later extensions of the setup to be compatible with other requirements such as X-ray fluorescence or tomographic measurements. The chamber shown in Fig. A.1 was designed within the Partnership for Soft Condensed Matter (PSCM) by Peter van der Linden with help from Pierre Lloria. Fig. A.1 shows the key components necessary for scanning X-ray diffraction at cryogenic temperatures in vacuum:

- A piezo-electric scanner and attached cryogenically cooled sample support,

- X-ray transmissive entrance and exit windows enabling small-angle and wideangle scattering experiments (exit window not shown in Fig. 4.4),

- A heat exchanger for efficient sample cooling,

- A connector box for tunneling of electrical signals from the piezo scanner, temperature sensors and heaters,

- A sample transfer connector for a future in-cryo sample exchange system.

The chamber is foreseen to operate at a vacuum pressure of $1 \times 10^{-6}$ mbar and 
temperatures below $100 \mathrm{~K}$. Since the main objective of the proposed project would be to facilitate cryogenic experiments on biological samples such as biological cells or tissues, the sample support is designed to be ideally suited for silicon nitride windows $(5 \mathrm{~mm} \times 5 \mathrm{~mm}$ frame size $)$ or TEM grids which are already heavily used in such experiments. A design for the sample support has to meet several important requirements: (i) The piezo-electric scanner should be thermally isolated from the cryogenic cooling unit, (ii) it should prevent too high lateral forces on the scanner during cooling, and (iii) should ideally be very compact. To meet these requirements, high-quality engineering plastic such as Torlon 4203L polyamide-imide would be suitable as isolating material. The design is symmetric to minimize resulting torques during cooling. We have conducted thermal simulations to ensure that the proposed design is capable of meeting the above mentioned requirements. As a candidate model for the piezo, we have chosen the PI P-733.2 two-axis scanning stage (Physik Instrumente (PI) GmbH \& Co. KG), as it provides a large aperture $(4 \mathrm{~cm} \times 4 \mathrm{~cm})$ for transmission experiments and a suitable scanning range of $100 \mu \mathrm{m}$. The second basic element is the cryogenic unit. It is equipped with two symmetrically placed heat exchangers that should be manufactured out of copper. Cold shields are needed to protect the piezo scanner against radiative cooling. Sample cooling is facilitated by two flexible copper braids that are attached to the heat exchangers and the sample support (not shown in Fig. A.1). This unit also provides connections for vacuum, sample transfer, and $\mathrm{LN}_{2}$ inlet and outlet. Finally, the cryogenic unit should be equipped with a connector box that contains electrical feedthroughs for the piezo scanner, heating elements and sensors.

An initial test of the chamber will be performed in 2018.

Flighttube for scanning diffraction experiments Inspired by the original design of a small, funnel-shaped and He-flushed flighttube available at the endstation ID13 of the ESRF, we have designed a similar flighttube for the use at the modular nanodiffraction and imaging setup GINIX at beamline P10, DESY. The flighttube was sketched in a 3D drawing studio such that it could be exported and $3 \mathrm{D}$ printed. A beamstop can be inserted into and removed from the interior of the flighttube and the exit window can be easily replaced since the large polypropylene or Kapton window can be pierced by the intense focused Xray beam. To prevent a too fast degradation of the window the beam is therefore blocked by the beamstop before the exit window. The assembly procedure and the $3 \mathrm{D}$ drawings are available by the author on demand. A final printed version of the flighttube and the corresponding 3D sketch are shown in Fig. A.2. The flighttube 


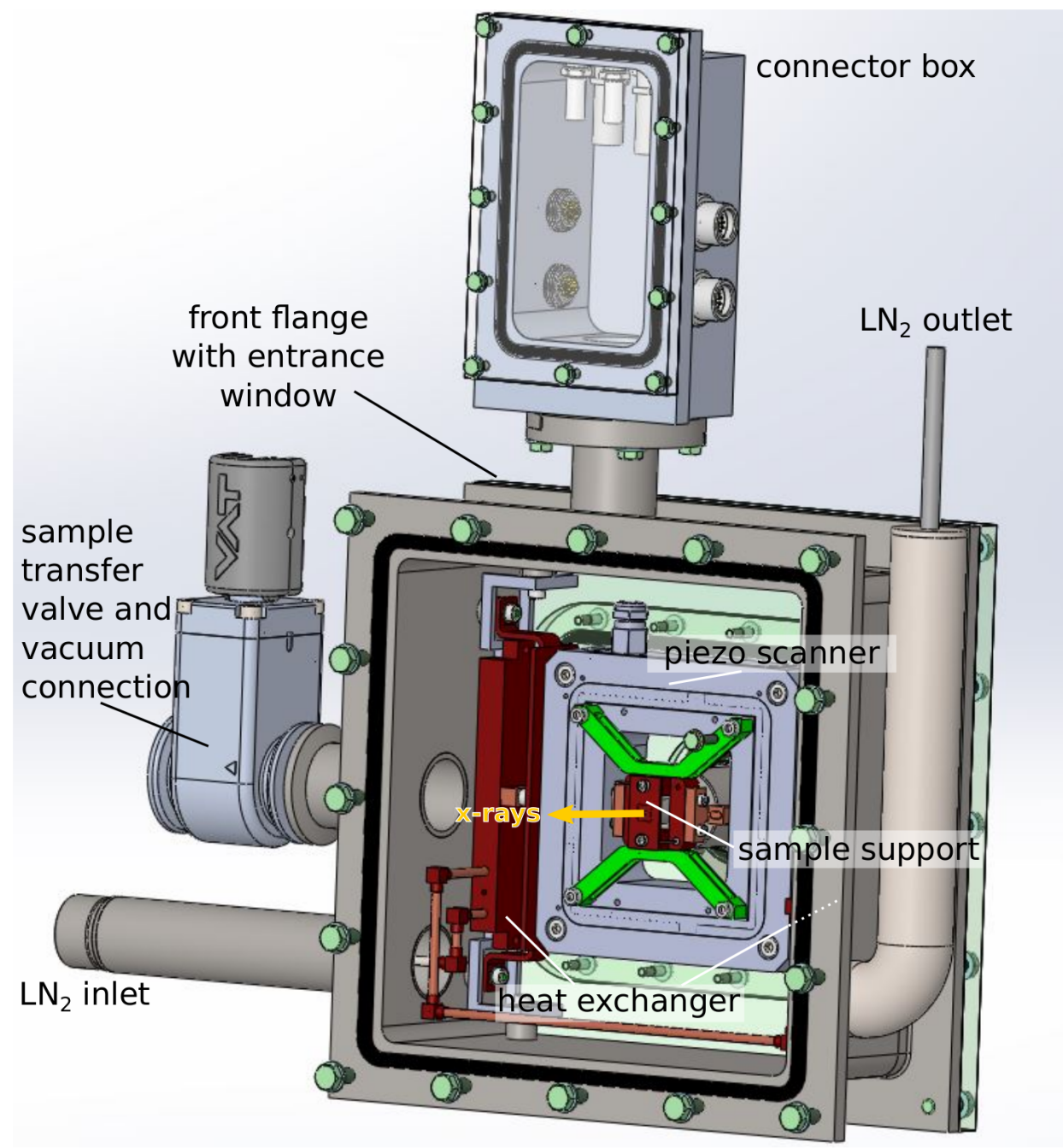

Figure A.1.: Assembled cryo-chamber for scanning X-ray diffraction. The sample will be placed on the sample support, which is cooled by two attached copper barbed wires (not shown) connected to two heat exchange units. The cabling of the piezo scanner is guided into a connector box that offers multiple electrical feedthroughs for the scanner and additional feedthrough options for thermal sensors and heating elements. The piezo scanner is protected against cooling by a cold shield (Kapton with resistive elements). The exit window is hidden in the figure for visual clarity. The direction of the X-ray beam is indicated in yellow. 
was printed using polylactic acid (PLA) with a standard, commercially available 3D printer (MakerBot Replicator 2, MakerBot). The flighttube was designed with Autodesk Inventor (Autodesk). The beamstop was built using an internal protocol and requires gluing of the beamstop material (typically lead wire) onto a glass capillary. The glass capillary is then inserted into a cylindrical mount. A protocol for beamstop manufacturing is available by the author on demand.

The assembly process of the $3 \mathrm{D}$ printed parts and the mounting of the flighttube can be summarized as follows:

1. Remove residual PLA (support structures) from all 3D printed parts.

2. Insert a screw in the beamstop holder (square piece of the inner ring). This is used to fix the beamstop.

3. Insert a beamstop into the holder of the inner ring.

4. Align the beamstop in the inner ring. For this purpose, a laser can be used to define the optical axis.

5. Screw the helium feedthrough into the inlet and outlet ports (due to inaccurate printing margins the openings might have to be slightly dilated using a $6 \mathrm{~mm}$ drill).

6. Seal the helium feedthrough using insulating tape.

7. Glue the entrance window with two component adhesive into the slot in the $3 \mathrm{D}$ print.

8. Insert an O-ring into the fitting.

9. Squeeze the inner ring with the mounted beamstop into the flighttube. You might want to use nailpolish, glue or a screw to fix the ring.

10. Finally, place the polypropylene window onto the O-ring and place the aluminium ring on top. Use screws and nuts to fix the aluminium ring and to tighten the seal.

11. Glue the flighttube into its beamline holder.

12. Insert the holder into a corresponding mount. We have used an optomechanical mount for precise adjustment of the beamstop tilt angles (e.g. ULMTILT, Newport Corporation, USA).

13. Fix the mount onto an aluminium frame.

14. Insert the flighttube into the beamline.

15. Attach the helium supply to a flowmeter.

16. Connect the flowmeter to the helium inlet of the flighttube. 
17. Connect the helium outlet to a bottle filled with water and seal the outlet using a metal clip. A tube should reach into the water such that the water level can generate an adjustable counter-pressure against the gas pressure.

18. Slowly start helium filling. The polypropylene window should bulge outwards and bubbles should appear inside the water-filled bottle.

The flighttube has been assembled and commissioned at the GINIX setup. The impact of the beamstop and the helium filling on the scattering signal was seperately tested as shown in Fig. A.3. First, the background level without the flighttube was recorded. Then, the flighttube was inserted but not yet flushed with helium, which already greatly reduced the background level, see Fig. A.3(B). Then, in Fig. A.3(C), the beamstop before the detector was removed and, lastly, the signal level could be further reduced by filling the flighttube with helium gas, see Fig. A.3(D). 

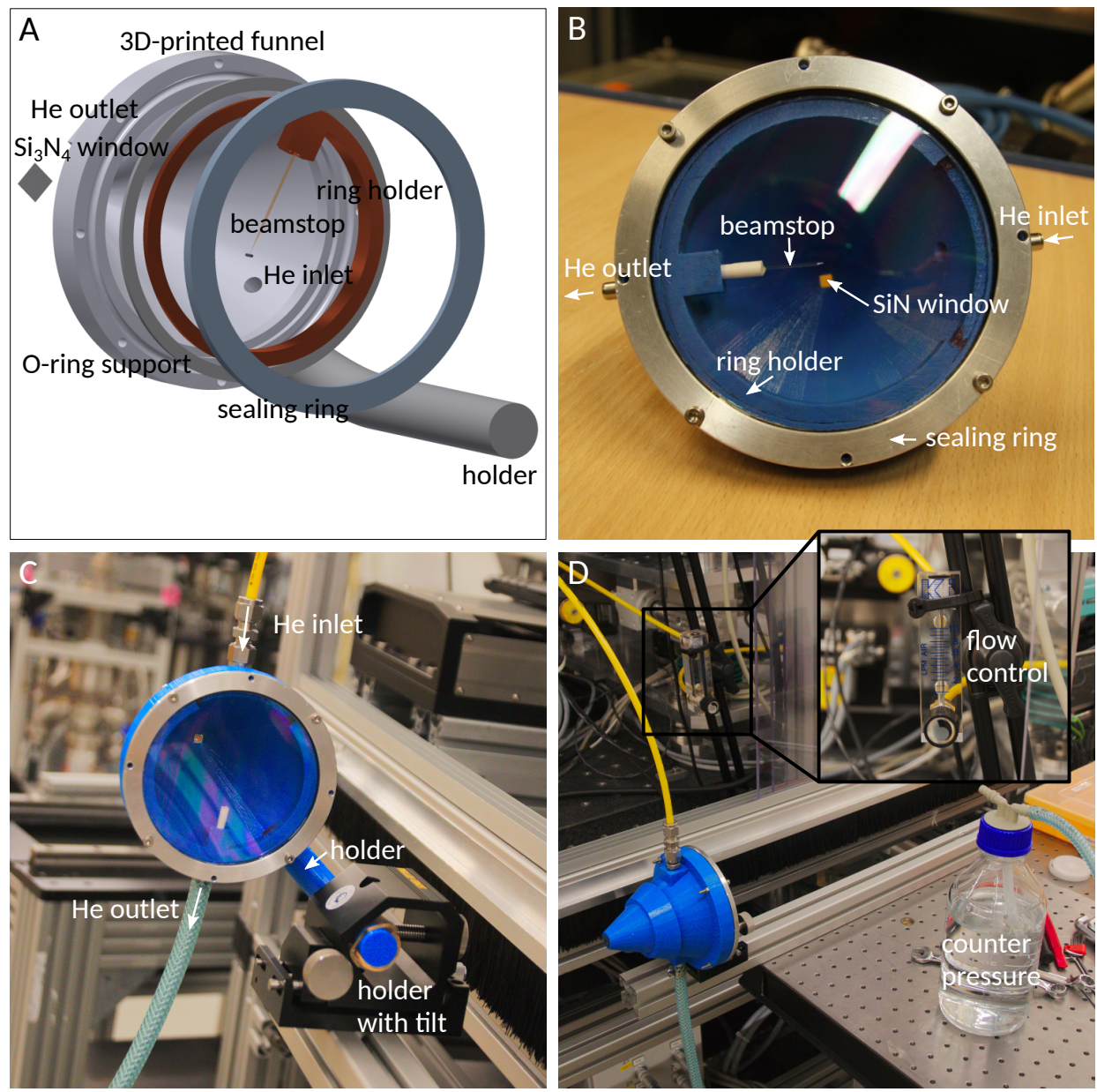

Figure A.2.: A 3D-printed flighttube for scanning X-ray diffraction experiments. In operation, the flighttube is flushed with helium to minimize background scattering. A beamstop is placed into the flighttube to remove the unscattered ray before it reaches the exit window. The overall flighttube design has been adapted from Manfred Burghammer and Britta Weinhausen, ESRF. (A) 3D-drawing of the flighttube. (B) Photograph of the assembled flighttube. For a description of the components and the assembly procedure, see the main text. (C) Photograph of an assembled flighttube attached to He-supply and mounted onto a holder such that the device can be inserted 'as is' into the experimental setup. (D) Different view on the assembled flighttube. The He outlet through a water-filled bottle is used to counterbalance the gas pressure and thus to pressurize the flighttube chamber. The helium gas pressure can be regulated and monitored using the flow control device shown in the inset of $(D)$. 

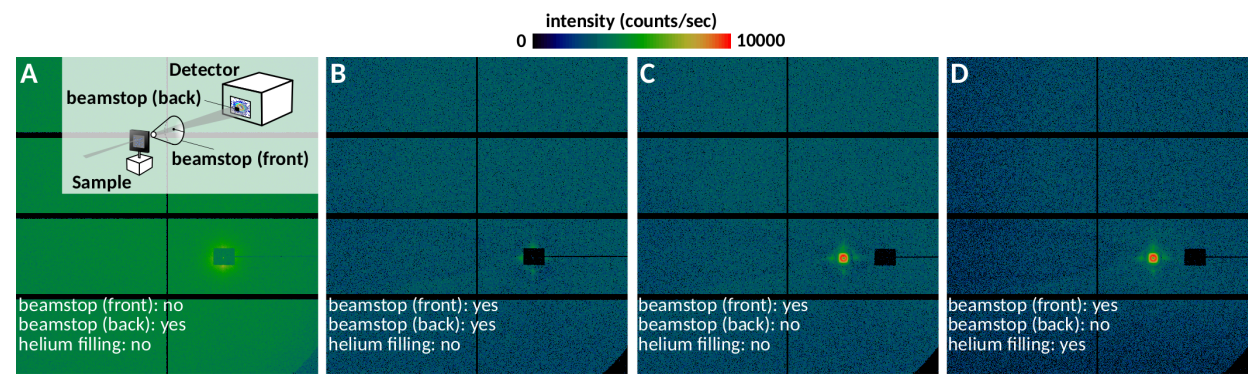

Figure A.3.: Flighttube commissioning. Background signal without a sample in place was recorded (A) with no flighttube in place and a beamstop placed shortly before the detector, $(B)$ with the flighttube in place but without helium and with a rear beamstop, $(C)$ as in (B) but without a beamstop directly before the detector, and $(D)$ as in $(C)$ but with the flighttube filled with helium which could further reduce the background scattering level. 


\section{B. Recommendations on the data analysis of scanning diffraction data}

In many instances, the analysis of scattering data can be obscured by artifacts that arise due to experimental difficulties or unknown parameters. This chapter explores some common pitfalls in the analysis of scattering data. It is not attempted to provide a complete description of all experimental factors that can be corrected for in subsequent analysis steps but to raise awareness by the experimenter that the amount of software-side correction can and should be minimized by careful planning of the experiment.

Beamstops Beamstops are a necessary evil in diffraction experiments as they are required to lower background levels but can in many instances lead to spurious scattering that can overlay the small-angle diffraction signal. In many cases, beamstops are prepared from lead or tungsten wire and glued onto thin glass capillaries. Glue and glass absorb a marginal amount of the scattering signal. Fortunately, absorption by the glass capillary and glue can be compensated for. First, background scattering should be accumulated to resonable signal levels, yielding an average pattern $I_{\text {avg }}$. Then, the background without the capillary can be integrated azimutally and in a second step be reinterpolated onto the entire detector to yield a filtered background $I_{\text {interp }}$. Each diffraction pattern that is to be analyzed can then be multiplied by $I_{\text {interp }} / I_{\text {avg }}$. An example of such a correction is shown in Fig. B.1.

Interpolation of missing data Missing data due to modular gaps, beamstops and other factors can in many cases be interpolated. Several measures can be taken to interpolate missing data. Since many experiments presented here record solely the small-angle scattering signal, the observed pattern should be point symmetric with respect to the primary beam position and one simple way to fill in data gaps is by copying data from the opposite side of the primary beam position. This approach has been termed 'healing' in the literature [240] and is implemented in the nanodiffraction toolbox in the heal routine. An example for a healed diffraction 


\section{Recommendations on the data analysis of scanning diffraction data}

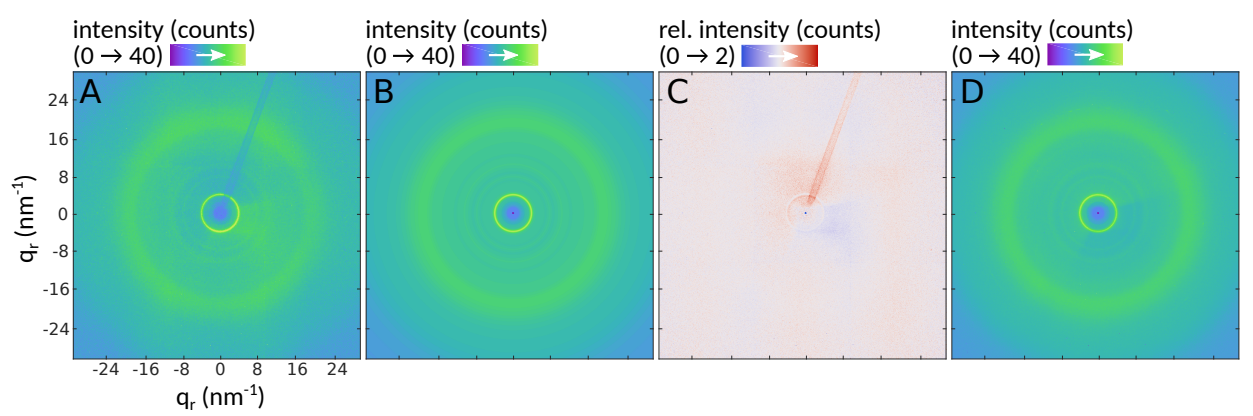

Figure B.1.: Correction for semi-transparent objects. In (A) a glass capillary is used as a beamstop mount. The region without the glass capillary was azimuthally averaged and re-interpolated onto the $2 d$ detector shown in (B). (C) Correction image resulting from dividing images $(A)$ and (B). (D) Average of 100 frames where each frame was multiplied with the correction image in $(C)$ before the averaging, confirming successfull removal of the beamstop.

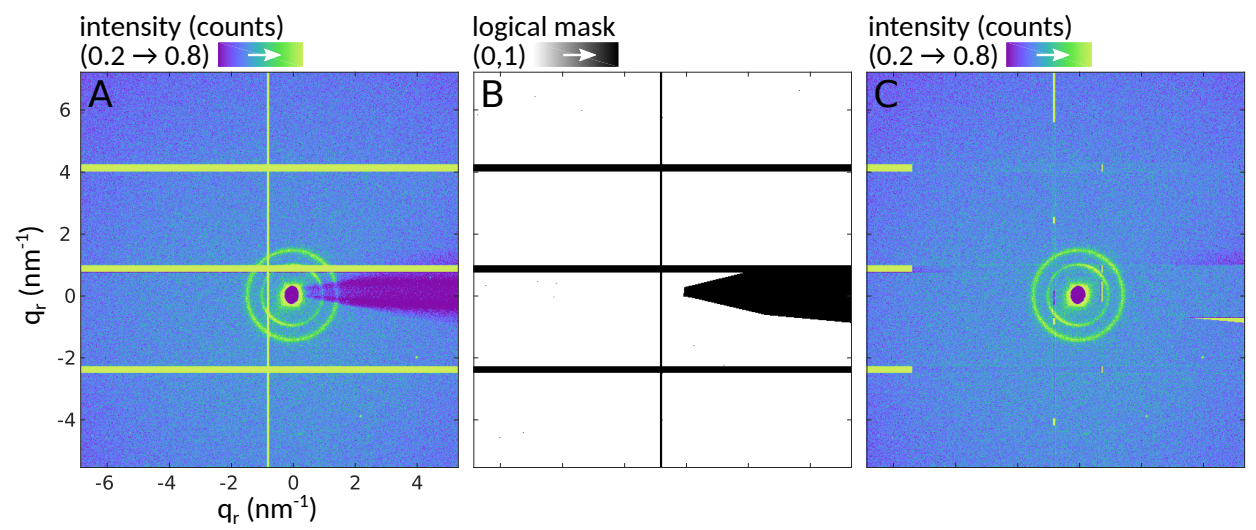

Figure B.2.: 'Healing' of scattering images. In (A) parts of the powder reflections could not be measured due to intermodular detector gaps. Furthermore the beamstop interferes with the powder rings. At low scattering angles, point symmetry of the diffraction pattern allows for copying of valid datapoints into invalid pixels on the opposite side of the primary beam location. Invalid pixels are identified in $(B)$. The diffraction pattern shown after the copy operation $(C)$ shows an almost completely recovered small-angle diffraction signal.

pattern is shown in Fig. B.2. It is advisable to choose the location of the primary beam with great care such that healing can be avoided or at least be used most efficiently. Semi-transparent beamstop holders should not be corrected for by healing but instead by a separate correction as described in the previous paragraph. 


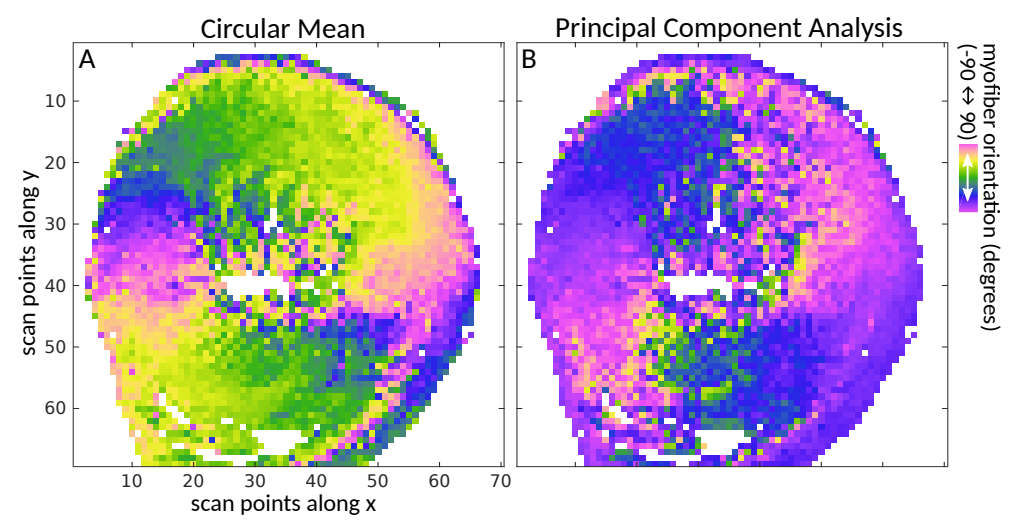

Figure B.3.: Myofiber orientation estimation in a histological section (short-axis view) of a mouse heart. (A) Circular mean. Myofiber orientation appears to run circular around the left ventricle of the heart. (B). Principal component analysis. The same background as in (A) was used. The orientation is strongly biased towards 80 degrees due to scattering from the beamstop into this direction.

Orientation analysis of the scattering distribution Orientation analysis of the scattering distribution relies in many cases on a determination of the maximum in the radially integrated intensity $I(\phi)$, averaged over a certain $q_{r}$-range. For circular data, we have used the circular mean and variance to characterize the azimuthal scattering distribution. The circular mean is in this respect indicating the orientation of the scattering signal. However, a similar (in many cases almost identical) result can be achieved by performing a principal component analysis on the $2 \mathrm{D}$ scattering pattern. This yields a different definition of the anisotropy of the scattering in contrast to the variance of the signal but was shown to give almost identical results. However, in the case when an anisotropic scattering signal is superimposed onto the diffraction pattern that is to be analyzed, it is preferable to use the circular mean. A comparison between the two methods is shown in Fig. B.3. For this example, a transverse histological section of a mouse heart was analyzed. The PCA result in (B) is strongly biased into the direction of the beamstop scattering. $2 \mathrm{D}$ background subtraction is insufficient in this case to remove the artifacts introduced by the spurious beamstop scattering. The same background was used for the calculation in (A). However, the background was radially integrated before it was subtracted which reduces noise in the data and increases the robustness of the method. 
Calibration of the detector distance and primary beam position The detector distance and primary beam position is routinely determined from measurements with calibration standards. In the experiments described here, we have frequently used silver behenate $(\mathrm{AgBh})$ and corundum $\left(\mathrm{Al}_{2} \mathrm{O}_{3}\right)$ for this purpose. For reasonable signal levels without damaging the silver behenate or corundum, we have laterally scanned a glass capillary containing the calibration standard with exposure times below one second per point and collected around 100 scattering patterns per detector distance. The average scattering pattern was calculated in Matlab (Mathworks Inc.) using the nanodiffraction toolbox and exported as '.tif', which was converted to the ESRF-custom '.edf' format using the python fabio package. The detector distance was then calibrated using the pyFAI-calib package. If multiple calibrations were performed at different detector distances, the energy of the radiation can also be determined. The linear relation between the actual movement of the detector motor and the determined detector distance should ideally be one. The assumed energy of the radiation should be divided by the slope of the linear regression to yield the correct energy of the radiation. 


\section{Bibliography}

1. Holmes, K. C. \& Rosenbaum, G. How X-ray Diffraction with Synchrotron Radiation Got Started. Journal of Synchrotron Radiation 5, 147-153 (1998).

2. Maddox, B. Rosalind Franklin: The Dark Lady of DNA (Harper Perennial, 2003).

3. Watson, J. D. \& Crick, F. H. C. Molecular Structure of Nucleic Acids: A Structure for Deoxyribose Nucleic Acid. Nature 171, 737-738 (1953).

4. Huxley, A. F. \& Niedergerke, R. Structural Changes in Muscle During Contraction: Interference Microscopy of Living Muscle Fibres. Nature 173, 971973 (1954).

5. Huxley, H. \& Hanson, J. Changes in the Cross-Striations of Muscle during Contraction and Stretch and their Structural Interpretation. Nature 173, 973-976 (1954).

6. Rosenbaum, G., Holmes, K. C. \& Witz, J. Synchrotron Radiation as a Source for X-ray Diffraction. Nature 230, 434-437 (1971).

7. Leigh, J. B. \& Rosenbaum, G. Synchrotron X-Ray Sources: A New Tool in Biological Structural and Kinetic Analysis. Annual Review of Biophysics and Bioengineering 5, 239-270 (1976).

8. Huxley, H. E., Faruqi, A. R., Bordas, J., Koch, M. H. J. \& Milch, J. R. The use of synchrotron radiation in time-resolved X-ray diffraction studies of myosin layer-line reflections during muscle contraction. Nature 284, 140 143 (1980).

9. Phillips, J. C., Wlodawer, A., Yevitz, M. M. \& Hodgson, K. O. Applications of Synchrotron Radiation to Protein Crystallography: Preliminary Results. Proceedings of the National Academy of Sciences 73, 128-132 (1976).

10. Huxley, A. F. \& Simmons, R. M. Proposed Mechanism of Force Generation in Striated Muscle. Nature 233, 533 (1971).

11. Piazzesi, G., Reconditi, M., Linari, M., Lucii, L., Sun, Y.-B., Narayanan, T., Boesecke, P., Lombardi, V. \& Irving, M. Mechanism of force generation by myosin heads in skeletal muscle. Nature 415, 659-662 (2002). 
12. Spudich, J. A. The myosin swinging cross-bridge model. Nature Reviews Molecular Cell Biology 2, 387 (2001).

13. Reconditi, M. et al. The myosin motor in muscle generates a smaller and slower working stroke at higher load. Nature 428, 578-581 (2004).

14. Linari, M., Brunello, E., Reconditi, M., Fusi, L., Caremani, M., Narayanan, T., Piazzesi, G., Lombardi, V. \& Irving, M. Force generation by skeletal muscle is controlled by mechanosensing in myosin filaments. Nature $\mathbf{5 2 8}$, 276-279 (2015).

15. Alberts, B., Johnson, A., Lewis, J., Raff, M., Roberts, K. \& Walter, P. Molecular Biology of the Cell Fifth edition (Garland Science, 2012).

16. Lymn, R. W. \& Taylor, E. W. Mechanism of adenosine triphosphate hydrolysis by actomyosin. Biochemistry 10, 4617-4624 (1971).

17. Irving, T. C., Konhilas, J., Perry, D., Fischetti, R. \& de Tombe, P. P. Myofilament lattice spacing as a function of sarcomere length in isolated rat myocardium. American Journal of Physiology-Heart and Circulatory Physiology 279, H2568-H2573 (2000).

18. Vă̈nshteĭn, B. K. Diffraction of X-rays by chain molecules (Elsevier, 1966).

19. Cantor, C. R. \& Schimmel, P. R. Biophysical Chemistry Part II: Techniques for the study of biological structure and function (W. H. Freeman and Company, 1980).

20. Angelini, T. E., Liang, H., Wriggers, W. \& Wong, G. C. L. Direct observation of counterion organization in F-actin polyelectrolyte bundles. The European Physical Journal E 16, 389-400 (2005).

21. Prodanovic, M., Irving, T. C. \& Mijailovich, S. M. X-ray diffraction from nonuniformly stretched helical molecules. Journal of Applied Crystallography 49, 784-797 (2016).

22. Huxley, H. E. \& Brown, W. The Low-angle X-ray Diagram of Vertebrate Striated Muscle and its Behaviour during Contraction and Rigor. Journal of Molecular Biology 30, 383-434 (1967).

23. Yagi, N., O’Brien, E. J. \& Matsubara, I. Changes of thick filament structure during contraction of frog striated muscle. Biophysical Journal 33, $121-137$ (1981).

24. Knupp, C. \& Squire, J. M. HELIX: a helical diffraction simulation program. Journal of Applied Crystallography 37, 832-835 (2004). 
25. Reconditi, M., Caremani, M., Pinzauti, F., Powers, J. D., Narayanan, T., Stienen, G. J. M., Linari, M., Lombardi, V. \& Piazzesi, G. Myosin filament activation in the heart is tuned to the mechanical task. Proceedings of the National Academy of Sciences 114, 3240-3245 (2017).

26. Ait-Mou, Y., Hsu, K., Farman, G. P., Kumar, M., Greaser, M. L., Irving, T. C. \& de Tombe, P. P. Titin strain contributes to the Frank-Starling law of the heart by structural rearrangements of both thin- and thick-filament proteins. Proceedings of the National Academy of Sciences 113, 2306-2311 (2016).

27. Stangl, J., Mocuta, C., Chamard, V. \& Carbone, D. Nanobeam X-ray Scattering: Probing Matter at the Nanoscale (Wiley-VCH Verlag GmbH \& Co. KGaA, 2014).

28. Lichtenegger, H., Müller, M., Paris, O., Riekel, C. \& Fratzl, P. Imaging of the helical arrangement of cellulose fibrils in wood by synchrotron X-ray microdiffraction. Journal of Applied Crystallography 32, 1127-1133 (1999).

29. Müller, M. Synchrotron Radiation X-Ray Scattering Techniques for Studying the Micro- and Nanostructure of Wood and their Relation to the Mechanical Properties in Advances in Materials Science of Wood 599 (Trans Tech Publications, 2009), 107-125.

30. Storm, S., Ogurreck, M., Laipple, D., Krywka, C., Burghammer, M., Di Cola, E. \& Müller, M. On radiation damage in FIB-prepared softwood samples measured by scanning X-ray diffraction. Journal of Synchrotron Radiation 22, 267-272 (2015).

31. Merk, V., Berg, J. K., Krywka, C. \& Burgert, I. Oriented Crystallization of Barium Sulfate Confined in Hierarchical Cellular Structures. Crystal Growth E Design 17, 677-684 (2017).

32. Wagermaier, W., Gupta, H. S., Gourrier, A., Paris, O., Roschger, P., Burghammer, M., Riekel, C. \& Fratzl, P. Scanning texture analysis of lamellar bone using microbeam synchrotron X-ray radiation. Journal of Applied Crystallography 40, 115-120 (2007).

33. Rinnerthaler, S., Roschger, P., Jakob, H. F., Nader, A., Klaushofer, K. \& Fratzl, P. Scanning Small Angle X-ray Scattering Analysis of Human Bone Sections. Calcified Tissue International 64, 422-429 (1999). 
34. Gourrier, A., Li, C., Siegel, S., Paris, O., Roschger, P., Klaushofer, K. \& Fratzl, P. Scanning small-angle X-ray scattering analysis of the size and organization of the mineral nanoparticles in fluorotic bone using a stack of cards model. Journal of Applied Crystallography 43, 1385-1392 (2010).

35. Granke, M., Gourrier, A., Rupin, F., Raum, K., Peyrin, F., Burghammer, M., Saïed, A. \& Laugier, P. Microfibril Orientation Dominates the Microelastic Properties of Human Bone Tissue at the Lamellar Length Scale. PLoS One 8, e58043 (2013).

36. Giannini, C. et al. Scanning SAXS-WAXS microscopy on osteoarthritisaffected bone - an age-related study. Journal of Applied Crystallography 47, 110-117 (2014).

37. Bukreeva, I. et al. High-Resolution X-Ray Techniques as New Tool to Investigate the 3D Vascularization of Engineered-Bone Tissue. Frontiers in Bioengineering and Biotechnology 3, 133 (2015).

38. Georgiadis, M., Guizar-Sicairos, M., Zwahlen, A., Trüssel, A. J., Bunk, O., Müller, R. \& Schneider, P. 3D scanning SAXS: A novel method for the assessment of bone ultrastructure orientation. Bone 71, 42-52 (2015).

39. Liebi, M., Georgiadis, M., Menzel, A., Schneider, P., Kohlbrecher, J., Bunk, O. \& Guizar-Sicairos, M. Nanostructure surveys of macroscopic specimens by small-angle scattering tensor tomography. Nature 527, 349-352 (2015).

40. Schaff, F., Bech, M., Zaslansky, P., Jud, C., Liebi, M., Guizar-Sicairos, M. \& Pfeiffer, F. Six-dimensional real and reciprocal space small-angle X-ray scattering tomography. Nature 527, 353-356 (2015).

41. Weinhausen, B., Nolting, J.-F., Olendrowitz, C., Langfahl-Klabes, J., Reynolds, M., Salditt, T. \& Köster, S. X-ray nano-diffraction on cytoskeletal networks. New Journal of Physics 14, 085013 (2012).

42. Weinhausen, B., Saldanha, O., Wilke, R. N., Dammann, C., Priebe, M., Burghammer, M., Sprung, M. \& Köster, S. Scanning X-Ray Nanodiffraction on Living Eukaryotic Cells in Microfluidic Environments. Physical Review Letters 112, 088102 (2014).

43. Priebe, M., Bernhardt, M., Blum, C., Tarantola, M., Bodenschatz, E. \& Salditt, T. Scanning X-Ray Nanodiffraction on Dictyostelium discoideum. Biophysical Journal 107, 2662 -2673 (2014). 
44. Bernhardt, M., Priebe, M., Osterhoff, M., Wollnik, C., Diaz, A., Salditt, T. \& Rehfeldt, F. X-Ray Micro- and Nanodiffraction Imaging on Human Mesenchymal Stem Cells and Differentiated Cells. Biophysical Journal 110, 680-690 (2016).

45. Pelz, P., Guizar-Sicairos, M., Thibault, P., Johnson, I., Holler, M. \& Menzel, A. On-the-fly scans for X-ray ptychography. 105, 251101 (2014).

46. Nicolas, J.-D., Bernhardt, M., Markus, A., Alves, F., Burghammer, M. \& Salditt, T. Scanning X-ray diffraction on cardiac tissue: automatized data analysis and processing. Journal of Synchrotron Radiation 24, 1163-1172 (2017).

47. Jensen, T. H., Bech, M., Bunk, O., Menzel, A., Bouchet, A., Duc, G. L., Feidenhans'l, R. \& Pfeiffer, F. Molecular X-ray computed tomography of myelin in a rat brain. Neuroimage 57, 124-129 (2011).

48. Kerman, B. E. et al. In vitro myelin formation using embryonic stem cells. Development 142, 2213-2225 (2015).

49. Kirschner, D. A., Hollingshead, C. J., Thaxton, C., Caspar, D. L. \& Goodenough, D. A. Structural states of myelin observed by x-ray diffraction and freeze-fracture electron microscopy. The Journal of Cell Biology 82, 140149 (1979).

50. Akers, C. K. \& Parsons, D. F. X-Ray Diffraction of Myelin Membrane: I. Optimal Conditions for Obtaining Unmodified Small Angle Diffraction Data from Frog Sciatic Nerve. Biophysical Journal 10, 101-115 (1970).

51. Elkes, J. \& Finean, J. B. X-ray Diffraction Studies on the Effect of Temperature on the Structure of Myelin in the Sciatic Nerve of the Frog. Experimental Cell Research 4, 69-81 (1953).

52. Inouye, H., Kuo, F.-H., Denninger, A. R., Weinhausen, B., Burghammer, M. \& Kirschner, D. A. Myelin structure in unfixed, single nerve fibers: Scanning X-ray microdiffraction with a beam size of $200 \mathrm{~nm}$. Journal of Structural Biology 200, 229-243 (2017).

53. Fahrni, C. J. Biological applications of X-ray fluorescence microscopy: exploring the subcellular topography and speciation of transition metals. Current Opinion in Chemical Biology 11, 121-127 (2007). 
54. Bohic, S., Murphy, K., Paulus, W., Cloetens, P., Salomé, M., Susini, J. \& Double, K. Intracellular Chemical Imaging of the Developmental Phases of Human Neuromelanin Using Synchrotron X-ray Microspectroscopy. Analytical Chemistry 80, 9557-9566 (2008).

55. Salditt, T. \& Dučić, T. in Super-Resolution Microscopy Techniques in the Neurosciences (eds Fornasiero, E. F. \& Rizzoli, S. O.) 257-290 (Humana Press, 2014).

56. Jellinger, K., Paulus, W., Grundke-Iqbal, I., Riederer, P. \& Youdim, M. B. H. Brain iron and ferritin in Parkinson's and Alzheimer's diseases. Journal of Neural Transmission - Parkinson's Disease and Dementia Section 2, 327340 (1990).

57. Dias, V., Junn, E. \& Mouradian, M. M. The Role of Oxidative Stress in Parkinson's Disease. Journal of Parkinson's Disease 3, 461-491 (2013).

58. Bourassa, M. W. \& Miller, L. M. Metal imaging in neurodegenerative diseases. Metallomics 4, 721-738 (2012).

59. Miao, J., Sayre, D. \& Chapman, H. N. Phase retrieval from the magnitude of the Fourier transforms of nonperiodic objects. Journal of the Optical Society of America A, Optics and Image Science 15, 1662-1669 (1998).

60. Rodenburg, J. M. \& Faulkner, H. M. L. A phase retrieval algorithm for shifting illumination. Applied Physics Letters 85, 4795-4797 (2004).

61. Giewekemeyer, K., Thibault, P., Kalbfleisch, S., Beerlink, A., Kewish, C. M., Dierolf, M., Pfeiffer, F. \& Salditt, T. Quantitative biological imaging by ptychographic x-ray diffraction microscopy. Proceedings of the National Academy of Sciences 107, 529-534 (2010).

62. Wilke, R. N., Priebe, M., Bartels, M., Giewekemeyer, K., Diaz, A., Karvinen, P. \& Salditt, T. Hard X-ray imaging of bacterial cells: nano-diffraction and ptychographic reconstruction. Optics Express 20, 19232-19254 (2012).

63. Salditt, T., Osterhoff, M., Krenkel, M., Wilke, R. N., Priebe, M., Bartels, M., Kalbfleisch, S. \& Sprung, M. Compound focusing mirror and X-ray waveguide optics for coherent imaging and nano-diffraction. Journal of Synchrotron Radiation 22, 867-878 (2015).

64. Bartels, M., Krenkel, M., Haber, J., Wilke, R. N. \& Salditt, T. X-Ray Holographic Imaging of Hydrated Biological Cells in Solution. Physical Review Letters 114, 048103 (2015). 
65. Krenkel, M. Cone-beam x-ray phase-contrast tomography for the observation of single cells in whole organs $\mathrm{PhD}$ thesis (University of Göttingen, 2015).

66. Teague, M. R. Deterministic phase retrieval: a Green's function solution. Journal of the Optical Society of America 73, 1434-1441 (1983).

67. Gureyev, T. E., Roberts, A. \& Nugent, K. A. Phase retrieval with the transport-of-intensity equation: matrix solution with use of Zernike polynomials. Journal of the Optical Society of America A, Optics and Image Science 12, 1932-1941 (1995).

68. Luke, D. R., Burke, J. V. \& Lyon, R. G. Optical Wavefront Reconstruction: Theory and Numerical Methods. SIAM Review 44, 169-224 (2002).

69. Allen, L. J. \& Oxley, M. P. Phase retrieval from series of images obtained by defocus variation. Optics Communications 199, 65-75 (2001).

70. Van der Schot, G. et al. Imaging single cells in a beam of live cyanobacteria with an X-ray laser. Nature Communications 6, 5704 (2015).

71. Reichardt, M., Frohn, J., Töpperwien, M., Nicolas, J.-D., Markus, A., Alves, F. \& Salditt, T. Nanoscale holographic tomography of heart tissue with $x$-ray waveguide optics in Developments in X-Ray Tomography XI (eds Müller, B. \& Wang, G.) 10391 (2017), 1039105.

72. Varray, F., Mirea, I., Langer, M., Peyrin, F., Fanton, L. \& Magnin, I. E. Extraction of the 3D local orientation of myocytes in human cardiac tissue using X-ray phase-contrast micro-tomography and multi-scale analysis. Medical Image Analysis 38, 117 -132 (2017).

73. Bernhardt, M., Nicolas, J.-D., Osterhoff, M., Mittelstädt, H., Reuss, M., Harke, B., Wittmeier, A., Sprung, M., Köster, S. \& Salditt, T. Correlative microscopy approach for biology using X-ray holography, X-ray scanning diffraction and STED microscopy. Nature Communications 9, 3641 (2018).

74. Huxley, H. E. A Personal View of Muscle and Motility Mechanisms. Annual Review of Physiology 58, 1-19 (1996).

75. Lombardi, V. et al. X-ray Diffraction Studies of the Contractile Mechanism in Single Muscle Fibres. Philosophical Transactions: Biological Sciences 359, 1883-1893 (2004).

76. Squire, J. M., Knupp, C., Roessle, M., AL-Khayat, H. A., Irving, T. C., Eakins, F., Mok, N.-S., Harford, J. J. \& Reedy, M. K. in (ed Sugi, H.) 45-60 (Springer US, 2005). 
77. Brunello, E., Bianco, P., Piazzesi, G., Linari, M., Reconditi, M., Panine, P., Narayanan, T., Helsby, W., Irving, M. \& Lombardi, V. Structural changes in the myosin filament and cross-bridges during active force development in single intact frog muscle fibres: stiffness and X-ray diffraction measurements. The Journal of Physiology 577, 971-984 (2006).

78. Wakabayashi, K., Sugimoto, Y., Takezawa, Y., Oshima, K., Matsuo, T., Ueno, Y. \& Irving, T. C. in Encyclopedia of Life Sciences (ELS) (John Wiley \& Sons, Ltd., 2010).

79. Töpperwien, M., Priebe, M. \& Salditt, T. Actin bundles cross-linked with $\alpha$ actinin studied by nanobeam X-ray diffraction. European Biophysics Journal 45, 383-392 (2015).

80. Giewekemeyer, K., Krüger, S. P., Kalbfleisch, S., Bartels, M., Beta, C. \& Salditt, T. X-ray propagation microscopy of biological cells using waveguides as a quasipoint source. American Physical Society 83, 23804 (2011).

81. Thibault, P., Dierolf, M., Menzel, A., Bunk, O., David, C. \& Pfeiffer, F. High-resolution scanning x-ray diffraction microscopy. Science 321, 379 382 (2008).

82. Beetz, T. \& Jacobsen, C. Soft X-ray radiation-damage studies in PMMA using a cryo-STXM. Journal of Synchrotron Radiation 10, 280-283 (2003).

83. Schneider, G. Cryo X-ray microscopy with high spatial resolution in amplitude and phase contrast. Ultramicroscopy 75, 85-104 (1998).

84. Weiß, D, Schneider, G, Niemann, B, Guttmann, P, Rudolph, D \& Schmahl, G. Computed tomography of cryogenic biological specimens based on X-ray microscopic images. Ultramicroscopy 84, 185-197 (2000).

85. Meyer-Ilse, W., Hamamoto, D., Nair, A., Lelièvre, S. A., Denbeaux, G., Johnson, L., Pearson, A. L., Yager, D., Legros, M. A. \& Larabell, C. A. High resolution protein localization using soft X-ray microscopy. Journal of Microscopy 201, 395-403 (2001).

86. Schneider, G., Guttmann, P., Heim, S., Rehbein, S., Mueller, F., Nagashima, K., Heymann, J. B., Muller, W. G. \& McNally, J. G. Three-dimensional cellular ultrastructure resolved by X-ray microscopy. Nature Methods 7, 985-987 (2010).

87. Huang, X. et al. Soft X-Ray Diffraction Microscopy of a Frozen Hydrated Yeast Cell. Physical Review Letters 103, 198101 (2009). 
88. Lima, E., Wiegart, L., Pernot, P., Howells, M., Timmins, J., Zontone, F. \& Madsen, A. Cryogenic X-Ray Diffraction Microscopy for Biological Samples. Physical Review Letters 103, 198102 (2009).

89. Jones, M. W. M. et al. Whole-cell phase contrast imaging at the nanoscale using Fresnel Coherent Diffractive Imaging Tomography. Scientific Reports 3, 2288 (2013).

90. Rodriguez, J. A. et al. Three-dimensional coherent X-ray diffractive imaging of whole frozen-hydrated cells. IUCrJ 2, 575 -583 (2015).

91. Takayama, Y. \& Yonekura, K. Cryogenic coherent X-ray diffraction imaging of biological samples at SACLA: a correlative approach with cryo-electron and light microscopy. Acta Crystallographica Section A 72, 179-189 (2016).

92. Lima, E., Diaz, A., Guizar-Sicairos, M., Gorelick, S., Pernot, P., Schleier, T. \& Menzel, A. Cryo-scanning x-ray diffraction microscopy of frozen-hydrated yeast. Journal of Microscopy 249, 1-7 (2013).

93. Deng, J., Vine, D. J., Chen, S., Nashed, Y. S. G., Jin, Q., Phillips, N. W., Peterka, T., Ross, R., Vogt, S. \& Jacobsen, C. J. Simultaneous cryo X-ray ptychographic and fluorescence microscopy of green algae. Proceedings of the National Academy of Sciences 112, 2314-2319 (2015).

94. Diaz, A. et al. Three-dimensional mass density mapping of cellular ultrastructure by ptychographic X-ray nanotomography. Journal of Structural Biology 192, 461-469 (2015).

95. De Jonge, M. D., Ryan, C. G. \& Jacobsen, C. J. X-ray nanoprobes and diffraction-limited storage rings: opportunities and challenges of fluorescence tomography of biological specimens. Journal of Synchrotron Radiation 21, 1031-1047 (2014).

96. Kalbfleisch, S., Neubauer, H., Krüger, S. P., Bartels, M., Osterhoff, M., Mai, D. D., Giewekemeyer, K., Hartmann, B., Sprung, M. \& Salditt, T. The Göttingen Holography Endstation of Beamline P10 at PETRA III/DESY. AIP Conference Proceedings 1365, 96-99 (2011).

97. Chen, H.-Y., Hoffmann, S. \& Salditt, T. X-ray beam compression by tapered waveguides. Applied Physics Letters 106, 194105 (2015).

98. Thibault, P., Dierolf, M., Kewish, C. M., Menzel, A., Bunk, O. \& Pfeiffer, F. Contrast mechanisms in scanning transmission x-ray microscopy. Physical Review A 80, 043813 (2009). 
99. Hémonnot, C. Y. J., Mauermann, M., Herrmann, H. \& Köster, S. Assembly of Simple Epithelial Keratin Filaments: Deciphering the Ion Dependence in Filament Organization. Biomacromolecules 16, 3313-3321 (2015).

100. Cloetens, P., Ludwig, W., Baruchel, J., Van Dyck, D., Van Landuyt, J., Guigay, J. P. \& Schlenker, M. Holotomography: Quantitative phase tomography with micrometer resolution using hard synchrotron radiation $\mathrm{x}$ rays. Applied Physics Letters 75, 2912-2914 (1999).

101. Krenkel, M., Markus, A., Bartels, M., Dullin, C., Alves, F. \& Salditt, T. Phase-contrast zoom tomography reveals precise locations of macrophages in mouse lungs. Scientific Reports 5, 9973 (2015).

102. Shen, Q., Bazarov, I. \& Thibault, P. Diffractive imaging of nonperiodic materials with future coherent X-ray sources. Journal of Synchrotron Radiation 11, $432-438$ (2004).

103. Howells, M. R. et al. An assessment of the resolution limitation due to radiation-damage in X-ray diffraction microscopy. Journal of Electron Spectroscopy and Related Phenomena 170, 4-12 (2009).

104. Kaulich, B., Thibault, P., Gianoncelli, A. \& Kiskinova, M. Transmission and emission x-ray microscopy: operation modes, contrast mechanisms and applications. Journal of Physics: Condensed Matter 23, 083002 (2011).

105. Fratzl, P., Jakob, H. F., Rinnerthaler, S., Roschger, P. \& Klaushofer, K. Position-Resolved Small-Angle X-ray Scattering of Complex Biological Materials. Journal of Applied Crystallography 30, 765-769 (1997).

106. Holt, M., Harder, R., Winarski, R. \& Rose, V. Nanoscale Hard X-Ray Microscopy Methods for Materials Studies. Annual Review of Materials Research 43, 183-211 (2013).

107. Bunk, O., Bech, M., Jensen, T. H., Feidenhans'l, R., Binderup, T., Menzel, A. \& Pfeiffer, F. Multimodal x-ray scatter imaging. New Journal of Physics 11, 123016 (2009).

108. Seidel, R., Gourrier, A., Burghammer, M., Riekel, C., Jeronimidis, G. \& Paris, O. Mapping fibre orientation in complex-shaped biological systems with micrometre resolution by scanning X-ray microdiffraction. Micron 39, 198-205 (2008).

109. Ice, G. E., Budai, J. D. \& Pang, J. W. L. The Race to X-ray Microbeam and Nanobeam Science. Science 334, 1234-1239 (2011). 
110. Mimura, H. et al. Breaking the $10 \mathrm{~nm}$ barrier in hard-X-ray focusing. Nature Physics 6, 122-125 (2010).

111. Vila-Comamala, J., Pan, Y., Lombardo, J. J., Harris, W. M., Chiu, W. K. S., David, C. \& Wang, Y. Zone-doubled Fresnel zone plates for high-resolution hard X-ray full-field transmission microscopy. Journal of Synchrotron Radiation 19, 705-709 (2012).

112. Da Silva, J. C., Pacureanu, A., Yang, Y., Bohic, S., Morawe, C., Barrett, R. \& Cloetens, P. Efficient concentration of high-energy x-rays for diffractionlimited imaging resolution. Optica 4, 492-495 (2017).

113. Sakdinawat, A. \& Attwood, D. Nanoscale X-ray imaging. Nature Photonics 4, 840-848 (2010).

114. Ryan, C. G. et al. Maia X-ray fluorescence imaging: Capturing detail in complex natural samples. Journal of Physics: Conference Series 499, 012002 (2014).

115. Johnson, I. et al. Capturing dynamics with Eiger, a fast-framing X-ray detector. Journal of Synchrotron Radiation 19, 1001-1005 (2012).

116. Tesch, W., Eidelman, N., Roschger, P., Goldenberg, F., Klaushofer, K. \& Fratzl, P. Graded Microstructure and Mechanical Properties of Human Crown Dentin. Calcified Tissue International 69, 147-157 (2001).

117. Märten, A., Fratzl, P., Paris, O. \& Zaslansky, P. On the mineral in collagen of human crown dentine. Biomaterials 31, 5479-5490 (2010).

118. Deyhle, H., Bunk, O. \& Müller, B. Nanostructure of healthy and cariesaffected human teeth. Nanomedicine: Nanotechnology, Biology and Medicine 7, 694-701 (2011).

119. Deyhle, H., White, S. N., Bunk, O., Beckmann, F. \& Müller, B. Nanostructure of carious tooth enamel lesion. Acta Biomaterialia 10, 355-364 (2014).

120. Karunaratne, A., Davis, G. R., Hiller, J., Esapa, C. T., Terrill, N. J., Brown, S. D. M., Cox, R. D., Thakker, R. V. \& Gupta, H. S. Hypophosphatemic rickets is associated with disruption of mineral orientation at the nanoscale in the flat scapula bones of rachitic mice with development. Bone 51, 553562 (2012).

121. Davies, R. J., Koenig, C., Burghammer, M. \& Riekel, C. On-axis microbeam wide- and small-angle scattering experiments of a sectioned poly(p-phenylene terephthalamide) fiber. Applied Physics Letters 92, 101903 (2008). 
122. Riekel, C., Burghammer, M., Dane, T. G., Ferrero, C. \& Rosenthal, M. Nanoscale Structural Features in Major Ampullate Spider Silk. Biomacromolecules 18, 231-241 (2017).

123. Zafeiropoulos, N. E., Davies, R. J., Roth, S. V., Burghammer, M., Schneider, K., Riekel, C. \& Stamm, M. Microfocus X-Ray Scattering Scanning Microscopy for Polymer Applications. Macromolecular Rapid Communications 26, 1547-1551 (2005).

124. Fratzl, P. Imaging techniques: Extra dimension for bone analysis. Nature 527, 308-309 (2015).

125. Seidel, R., Gourrier, A., Kerschnitzki, M., Burghammer, M., Fratzl, P., Gupta, H. S. \& Wagermaier, W. Synchrotron 3D SAXS analysis of bone nanostructure. Bioinspired, Biomimetic and Nanobiomaterials 1, 123-131 (2012).

126. Jensen, T. H., Bech, M., Bunk, O., Thomsen, M., Menzel, A., Bouchet, A., Duc, G. L., Feidenhans'l, R. \& Pfeiffer, F. Brain tumor imaging using small-angle x-ray scattering tomography. Physics in Medicine and Biology 56, 1717 (2011).

127. Grünewald, T. A., Rennhofer, H., Tack, P., Garrevoet, J., Wermeille, D., Thompson, P., Bras, W., Vincze, L. \& Lichtenegger, H. C. Photonenenergie als dritte Dimension bei der Analyse der kristallographischen Textur. Angewandte Chemie 128, 12376-12381 (2016).

128. Matsubara, I. X-Ray Diffraction Studies of the Heart. Annual Review of Biophysics and Bioengineering 9, 81-105 (1980).

129. Hubbell, J. H. \& Seltzer, S. M. Tables of X-Ray Mass Attenuation Coefficients and Mass Energy-Absorption Coefficients from $1 \mathrm{keV}$ to $20 \mathrm{MeV}$ for Elements $Z=1$ to 92 and 48 Additional Substances of Dosimetric Interest (version 1.4) [Online] Available: http://physics.nist.gov/xaamdi [2017, August, 1]. 2004.

130. Bernhardt, M., Nicolas, J.-D., Eckermann, M., Eltzner, B., Rehfeldt, F. \& Salditt, T. Anisotropic x-ray scattering and orientation fields in cardiac tissue cells. New Journal of Physics 19, 013012 (2017).

131. Kovesi, P. Good Colour Maps: How to Design Them. ArXiv e-prints (2015).

132. Konhilas, J. P., Irving, T. C. \& de Tombe, P. P. Myofilament Calcium Sensitivity in Skinned Rat Cardiac Trabeculae. Circulation Research 90, 59-65 (2002). 
133. Yagi, N. et al. X-ray Diffraction from a Left Ventricular Wall of Rat Heart. Biophysical Journal 86, 2286-2294 (2004).

134. Hammersley, A. P. FIT2D: An Introduction and Overview. ESRF Internal Report ESRF97HA02T (1997).

135. Kieffer, J. \& Karkoulis, D. PyFAI, a versatile library for azimuthal regrouping. Journal of Physics: Conference Series 425, 202012 (2013).

136. Shirai, M., Schwenke, D. O., Tsuchimochi, H., Umetani, K., Yagi, N. \& Pearson, J. T. Synchrotron Radiation Imaging for Advancing Our Understanding of Cardiovascular Function. Circulation Research 112, 209-221 (2013).

137. Matsubara, I, Maughan, D. W., Saeki, Y \& Yagi, N. Cross-bridge movement in rat cardiac muscle as a function of calcium concentration. The Journal of Physiology 417, 555-565 (1989).

138. Fratzl, P. Cellulose and collagen: from fibres to tissues. Current Opinion in Colloid $\&$ Interface Science 8, 32-39 (2003).

139. Fratzl, P. \& Weinkamer, R. Natures hierarchical materials. Progress in Materials Science 52, 1263-1334 (2007).

140. Lopes, L. R. \& Elliott, P. M. A straightforward guide to the sarcomeric basis of cardiomyopathies. Heart 100, 1916-1923 (2014).

141. Filik, J. et al. Processing two-dimensional X-ray diffraction and small-angle scattering data in DAWN 2. Journal of Applied Crystallography 50, 959-966 (2017).

142. Benecke, G. et al. A customizable software for fast reduction and analysis of large X-ray scattering data sets: applications of the new DPDAK package to small-angle X-ray scattering and grazing-incidence small-angle X-ray scattering. Journal of Applied Crystallography 47, 1797-1803 (2014).

143. Davies, R. J. A new batch-processing data-reduction application for X-ray diffraction data. Journal of Applied Crystallography 39, 267-272 (2006).

144. Bergamaschi, A., Medjoubi, K., Messaoudi, C., Marco, S. \& Somogyi, A. MMX-I: data-processing software for multimodal X-ray imaging and tomography. Journal of Synchrotron Radiation 23, 783-794 (2016).

145. Nicolas, J.-D., Bernhardt, M., Markus, A., Alves, F., Burghammer, M. \& Salditt, T. Scanning X-ray diffraction data on cardiac tissue. Zenodo, https://doi.org/10.5281/zenodo.1027712. 
146. Lutz-Bueno, V., Zhao, J., Mezzenga, R., Pfohl, T., Fischer, P. \& Liebi, M. Scanning-SAXS of microfluidic flows: nanostructural mapping of soft matter. Lab on a Chip 16, 4028-4035 (2016).

147. Hémonnot, C. Y. J. \& Köster, S. Imaging of Biological Materials and Cells by X-ray Scattering and Diffraction. ACS Nano 11, 8542-8559 (2017).

148. Nicolas, J.-D., Bernhardt, M., Krenkel, M., Richter, C., Luther, S. \& Salditt, T. Combined scanning X-ray diffraction and holographic imaging of cardiomyocytes. Journal of Applied Crystallography 50, 612-620 (2017).

149. Tiburcy, M. et al. Terminal Differentiation, Advanced Organotypic Maturation, and Modeling of Hypertrophic Growth in Engineered Heart Tissue. Circulation Research 109, 1105-1114 (2011).

150. Tiburcy, M. et al. Defined Engineered Human Myocardium With Advanced Maturation for Applications in Heart Failure Modeling and Repair. Circulation 135, 1832-1847 (2017).

151. Rother, J., Richter, C., Turco, L., Knoch, F., Mey, I., Luther, S., Janshoff, A., Bodenschatz, E. \& Tarantola, M. Crosstalk of cardiomyocytes and fibroblasts in co-cultures. Open Biology 5, 150038 (2015).

152. Richter, C., Christoph, J., Lehnart, S. E. \& Luther, S. in Optogenetics: Methods and Protocols (ed Kianianmomeni, A.) 293-302 (Springer, 2016).

153. Shen, K., Qi, J. \& Kam, L. C. Microcontact Printing of Proteins for Cell Biology. Journal of Visualized Experiments 22, e1065 (2008).

154. McDevitt, T. C., Angello, J. C., Whitney, M. L., Reinecke, H., Hauschka, S. D., Murry, C. E. \& Stayton, P. S. In vitro generation of differentiated cardiac myofibers on micropatterned laminin surfaces. Journal of Biomedical Materials Research 60, 472-479 (2002).

155. Nakasako, M. et al. KOTOBUKI-1 apparatus for cryogenic coherent X-ray diffraction imaging. Review of Scientific Instruments 84, 093705 (2013).

156. Chen, S. et al. The Bionanoprobe: hard X-ray fluorescence nanoprobe with cryogenic capabilities. Journal of Synchrotron Radiation 21, 66-75 (2014).

157. Hitchcock, A. P. Soft X-ray spectromicroscopy and ptychography. Journal of Electron Spectroscopy and Related Phenomena 200, 49-63 (2015).

158. Yusuf, M., Zhang, F., Chen, B., Bhartiya, A., Cunnea, K., Wagner, U., Cacho-Nerin, F., Schwenke, J. \& Robinson, I. K. Procedures for cryogenic Xray ptychographic imaging of biological samples. IUCrJ 4, 147-151 (2017). 
159. Meisburger, S., Warkentin, M., Chen, H., Hopkins, J., Gillilan, R., Pollack, L. \& Thorne, R. Breaking the Radiation Damage Limit with Cryo-SAXS. Biophysical Journal 104, 227-236 (2013).

160. Ekeberg, T. et al. Three-Dimensional Reconstruction of the Giant Mimivirus Particle with an X-Ray Free-Electron Laser. Physical Review Letters 114, 098102 (2015).

161. Seibert, M. M. et al. Femtosecond diffractive imaging of biological cells. Journal of Physics B: Atomic, Molecular and Optical Physics 43, 194015 (2010).

162. Hantke, M. F. et al. High-throughput imaging of heterogeneous cell organelles with an X-ray laser. Nature Photonics 8, 943-949 (2014).

163. Kimura, T. et al. Imaging live cell in micro-liquid enclosure by X-ray laser diffraction. Nature Communications 5, 3052 (2014).

164. Strelnikova, N., Sauter, N., Guizar-Sicairos, M., Göllner, M., Diaz, A., Delivani, P., Chacón, M., Tolić, I. M., Zaburdaev, V. \& Pfohl, T. Live cell $\mathrm{X}$-ray imaging of autophagic vacuoles formation and chromatin dynamics in fission yeast. Scientific Reports 7, 13775 (2017).

165. Denz, M. et al. Cyclic olefin copolymer as an X-ray compatible material for microfluidic devices. Lab on a Chip 18, 171-178 (2018).

166. Ghazal, A., Lafleur, J. P., Mortensen, K., Kutter, J. P., Arleth, L. \& Jensen, G. V. Recent advances in X-ray compatible microfluidics for applications in soft materials and life sciences. Lab on a Chip 16, 4263-4295 (2016).

167. deAlmeida, A. C., van Oort, R. J. \& Wehrens, X. H. T. Transverse Aortic Constriction in Mice. Journal of Visualized Experiments 38, e1729 (2010).

168. Toischer, K. et al. Differential Cardiac Remodeling in Preload Versus Afterload. Circulation 122, 993-1003 (2010).

169. Karlsson, U. \& Schultz, R. L. Fixation of the central nervous system for electron microscopy by aldehyde perfusion: I. Preservation with aldehyde perfusates versus direct perfusion with osmium tetroxide with special reference to membranes and the extracellular space. Journal of Ultrastructure Research 12, 160-186 (1965).

170. Schultz, R. L. \& Karlsson, U. Fixation of the central nervous system for electron microscopy by aldehyde perfusion: II. Effect of osmolarity, pH of perfusate, and fixative concentration. Journal of Ultrastructure Research $\mathbf{1 2}$, 187-206 (1965). 
171. Bernhardt, M., Nicolas, J.-D., Osterhoff, M., Mittelstädt, H., Reuss, M., Harke, B., Wittmeier, A., Sprung, M., Köster, S. \& Salditt, T. A Beamlinecompatible STED-Microscope for Combined Visible Light and X-Ray Studies on Biological Matter. submitted (2018).

172. Bertram, L. \& Tanzi, R. E. The genetic epidemiology of neurodegenerative disease. The Journal of Clinical Investigation 115, 1449-1457 (2005).

173. Hughes, A. J., Daniel, S. E., Ben-Shlomo, Y. \& Lees, A. J. The accuracy of diagnosis of parkinsonian syndromes in a specialist movement disorder service. Brain 125, 861-870 (2002).

174. Spillantini, M. G., Schmidt, M. L., Lee, V. M.-Y., Trojanowski, J. Q., Jakes, R. \& Goedert, M. $\alpha$-Synuclein in Lewy bodies. Nature 388, 839-840 (1997).

175. Dexter, D. T., Wells, F. R., Lee, A. J., Agid, F., Agid, Y., Jenner, P. \& Marsden, C. D. Increased Nigral Iron Content and Alterations in Other Metal Ions Occurring in Brain in Parkinson's Disease. Journal of Neurochemistry 52, 1830-1836 (1989).

176. Szczerbowska-Boruchowska, M., Krygowska-Wajs, A. \& Adamek, D. Elemental micro-imaging and quantification of human substantia nigra using synchrotron radiation based x-ray fluorescence - in relation to Parkinson's disease. Journal of Physics: Condensed Matter 24, 244104 (2012).

177. Carboni, E. \& Lingor, P. Insights on the interaction of alpha-synuclein and metals in the pathophysiology of Parkinson's disease. Metallomics 7, 395404 (2015).

178. Meiser, J., Weindl, D. \& Hiller, K. Complexity of dopamine metabolism. Cell Communication and Signaling 11, 34 (2013).

179. Sulzer, D. et al. Neuromelanin biosynthesis is driven by excess cytosolic catecholamines not accumulated by synaptic vesicles. Proceedings of the National Academy of Sciences 97, 11869-11874 (2000).

180. Zucca, F. A., Segura-Aguilar, J., Ferrari, E., Muñoz, P., Paris, I., Sulzer, D., Sarna, T., Casella, L. \& Zecca, L. Interactions of iron, dopamine and neuromelanin pathways in brain aging and Parkinson's disease. Progress in Neurobiology 155, 96-119 (2017).

181. Doria, M., Maugest, L., Moreau, T., Lizard, G. \& Vejux, A. Contribution of cholesterol and oxysterols to the pathophysiology of Parkinson's disease. Free Radical Biology and Medicine 101, 393-400 (2016). 
182. Vogt, S., Maser, J. \& Jacobsen, C. Data analysis for X-ray fluorescence imaging. Journal de Physique IV 104, 617-622 (2003).

183. Szczerbowska-Boruchowska, M. X-ray fluorescence spectrometry, an analytical tool in neurochemical research. X-Ray Spectrometry 37, 21-31 (2008).

184. Dučić, T., Quintes, S., Nave, K.-A., Susini, J., Rak, M., Tucoulou, R., Alevra, M., Guttmann, P. \& Salditt, T. Structure and composition of myelinated axons: A multimodal synchrotron spectro-microscopy study. Journal of Structural Biology 173, 202-212 (2011).

185. James, S. A. et al. Quantitative comparison of preparation methodologies for x-ray fluorescence microscopy of brain tissue. Analytical and Bioanalytical Chemistry 401, 853-864 (2011).

186. Dodani, S. C., Domaille, D. W., Nam, C. I., Miller, E. W., Finney, L. A., Vogt, S. \& Chang, C. J. Calcium-dependent copper redistributions in neuronal cells revealed by a fluorescent copper sensor and X-ray fluorescence microscopy. Proceedings of the National Academy of Sciences 108, 59805985 (2011).

187. Lynch, M. J., Raphael, S. S., Mellor, L. D., Spare, P. D. \& Inwood, M. J. Medical Laboratory Technology and Clinical Pathology Second edition (WB Saunders Co., 1969).

188. Fischer, A. H., Jacobson, K. A., Rose, J. \& Zeller, R. Fixation and Permeabilization of Cells and Tissues. Cold Spring Harbor Protocols 3, 1-2 (2008).

189. Solé, V. A., Papillon, E., Cotte, M., Walter, P. \& Susini, J. A multiplatform code for the analysis of energy-dispersive X-ray fluorescence spectra. Spectrochimica Acta Part B: Atomic Spectroscopy 62, 63-68 (2007).

190. Kirschner, D. A. \& Hollingshead, C. J. Processing for electron microscopy alters membrane structure and packing in myelin. Journal of Ultrastructure Research 73, 211-232 (1980).

191. Shaharabani, R., Ram-On, M., Avinery, R., Aharoni, R., Arnon, R., Talmon, Y. \& Beck, R. Structural Transition in Myelin Membrane as Initiator of Multiple Sclerosis. Journal of the American Chemical Society 138, 1215912165 (2016).

192. Joy, R. T. \& Finean, J. B. A comparison of the effects of freezing and of treatment with hypertonic solutions on the structure of nerve myelin. Journal of Ultrastructure Research 8, 264-282 (1963). 
193. Finean, J. B. The Nature and Stability of Nerve Myelin. International Review of Cytology 12, 303-336 (1962).

194. Worthington, C. R. \& Worthington, A. R. Effect of heat on frog sciatic nerve determined by X-ray diffraction. International Journal of Biological Macromolecules 3, 159-164 (1981).

195. Israelachvili, J. N. Intermolecular and Surface Forces (Academic Press, 2015).

196. Shieh, H. S., Hoard, L. G. \& Nordman, C. E. Crystal structure of anhydrous cholesterol. Nature 267, 287-289 (1977).

197. Davies, K. M. et al. Copper pathology in vulnerable brain regions in Parkinson's disease. Neurobiology of Aging 35, 858-866 (2014).

198. Surowka, A. D., Töpperwien, M., Bernhardt, M., Nicolas, J.-D., Osterhoff, M., Salditt, T., Adamek, D. \& Szczerbowska-Boruchowska, M. Combined in-situ imaging of structural organization and elemental composition of substantia nigra neurons in the elderly. Talanta 161, 368-376 (2016).

199. Zecca, L., Shima, T., Stroppolo, A., Goj, C., Battiston, G., Gerbasi, R., Sarna, T. \& Swartz, H. Interaction of neuromelanin and iron in substantia nigra and other areas of human brain. Neuroscience 73, 407-415 (1996).

200. Szczerbowska-Boruchowska, M., Lankosz, M., Ostachowicz, J., Adamek, D., Krygowska-Wajs, A., Tomik, B., Szczudlik, A., Simionovici, A. \& Bohic, S. Topographic and quantitative microanalysis of human central nervous system tissue using synchrotron radiation. X-Ray Spectrometry 33, 3-11 (2004).

201. Binolfi, A., Rasia, R. M., Bertoncini, C. W., Ceolin, M., Zweckstetter, M., Griesinger, C., Jovin, T. M. \& Fernández, C. O. Interaction of $\alpha$ Synuclein with Divalent Metal Ions Reveals Key Differences: A Link between Structure, Binding Specificity and Fibrillation Enhancement. Journal of the American Chemical Society 128, 9893-9901 (2006).

202. Paik, S. R., Shin, H.-J., Lee, J.-H., Chang, C.-S. \& Kim, J. Copper(II)induced self-oligomerization of $\alpha$-synuclein. Biochemical Journal 340, 821$828(1999)$.

203. Uversky, V. N., Li, J. \& Fink, A. L. Metal-triggered Structural Transformations, Aggregation, and Fibrillation of Human $\alpha$-Synuclein: A Possible Molecular Link Between Parkinson's Disease and Heavy Metal Exposure. Journal of Biological Chemistry 276, 44284-44296 (2001). 
204. Liu, J., Costantino, I., Venugopalan, N., Fischetti, R. F., Hyman, B. T., Frosch, M. P., Gomez-Isla, T. \& Makowski, L. Amyloid structure exhibits polymorphism on multiple length scales in human brain tissue. Scientific Reports 6, 33079 (2016).

205. Loomis, C. R., Shipley, G. G. \& Small, D. M. The phase behavior of hydrated cholesterol. Journal of Lipid Research 20, 525-35 (1979).

206. Cheng, D. et al. Lipid Pathway Alterations in Parkinson's Disease Primary Visual Cortex. PLoS One 6, e17299 (2011).

207. Li, Z., Zhang, J. \& Sun, H. Increased plasma levels of phospholipid in Parkinson's disease with mild cognitive impairment. Journal of Clinical Neuroscience 22, 1268-1271 (2015).

208. Zarrouk, A., Vejux, A., Mackrill, J., O'Callaghan, Y., Hammami, M., O'Brien, N. \& Lizard, G. Involvement of oxysterols in age-related diseases and ageing processes. Ageing Research Reviews 18, 148-162 (2014).

209. Bousquet, M., St-Amour, I., Vandal, M., Julien, P., Cicchetti, F. \& Calon, F. High-fat diet exacerbates MPTP-induced dopaminergic degeneration in mice. Neurobiology of Disease 45, 529-538 (2012).

210. Van Maarschalkerweerd, A., Vetri, V. \& Vestergaard, B. Cholesterol facilitates interactions between $\alpha$-synuclein oligomers and charge-neutral membranes. FEBS Letters 589, 2661-2667 (2015).

211. Miao, J., Charalambous, P., Kirz, J. \& Sayre, D. Extending the methodology of X-ray crystallography to allow imaging of micrometre-sized noncrystalline specimens. Nature 400, 342-344 (1999).

212. Gaffney, K. J. \& Chapman, H. N. Imaging Atomic Structure and Dynamics with Ultrafast X-ray Scattering. Science 316, 1444-1448 (2007).

213. Mancuso, A. P., Aquila, A., Borchers, G., Giewekemeyer, K. \& Reimers, N. Technical Design Report: Scientific Instrument Single Particles, Clusters, and Biomolecules (SPB), 1-232 (2013).

214. Seibert, M. M. et al. Single mimivirus particles intercepted and imaged with an X-ray laser. Nature 470, 78-82 (2011).

215. Ashkin, A. Trapping of Atoms by Resonance Radiation Pressure. Physical Review Letters 40, 729-732 (1978).

216. Ashkin, A \& Dziedzic, J. Optical trapping and manipulation of viruses and bacteria. Science 235, 1517-1520 (1987). 
217. Ashkin, A. Acceleration and Trapping of Particles by Radiation Pressure. Physical Review Letters 24, 156-159 (1970).

218. Cojoc, D., Ferrari, E., Garbin, V., Fabrizio, E. D., Amenitsch, H., Rappolt, M., Sartori, B., Laggner, P., Burghammer, M. \& Riekel, C. Scanning x-ray microdiffraction of optically manipulated liposomes. Applied Physics Letters 91, 234107 (2007).

219. Cojoc, D., Amenitsch, H., Ferrari, E., Santucci, S. C., Sartori, B., Rappolt, M., Marmiroli, B., Burghammer, M. \& Riekel, C. Local x-ray structure analysis of optically manipulated biological micro-objects. Applied Physics Letters 97, 244101 (2010).

220. Santucci, S. C., Cojoc, D., Amenitsch, H., Marmiroli, B., Sartori, B., Burghammer, M., Schoeder, S., DiCola, E., Reynolds, M. \& Riekel, C. Optical Tweezers for Synchrotron Radiation Probing of Trapped Biological and Soft Matter Objects in Aqueous Environments. Analytical Chemistry 83, 48634870 (2011).

221. Santucci, S. C., Amenitsch, H., Cojoc, D. \& Riekel, C. in Synchrotron Radiation and Structural Proteomics (eds Pechkova, E. \& Riekel, C.) 183-211 (Pan Stanford Publishing, 2011).

222. Gao, Y., Harder, R., Southworth, S., Guest, J., Scherer, N., Yan, Z., Ocola, L., Pelton, M. \& Young, L. Bragg diffraction from sub-micron particles isolated by optical tweezers. AIP Conference Proceedings 1741, 050010 (2016).

223. Sivaramakrishnan, S., Sung, J., Ali, M., Doniach, S., Flyvbjerg, H. \& Spudich, J. A. Combining Single-Molecule Optical Trapping and Small-Angle X-Ray Scattering Measurements to Compute the Persistence Length of a Protein ER/K $\alpha$-Helix. Biophysical Journal 97, 2993-2999 (2009).

224. Vergucht, E. et al. In vivo X-ray elemental imaging of single cell model organisms manipulated by laser-based optical tweezers. Scientific Reports 5, 9049 (2015).

225. Vergucht, E. et al. Methodological challenges of optical tweezers-based X-ray fluorescence imaging of biological model organisms at synchrotron facilities. Journal of Synchrotron Radiation 22, 1096-1105 (2015).

226. Guck, J., Ananthakrishnan, R., Moon, T. J., Cunningham, C. C. \& Käs, J. Optical Deformability of Soft Biological Dielectrics. Physical Review Letters 84, 5451-5454 (2000). 
227. Boyde, L., Chalut, K. J. \& Guck, J. Interaction of Gaussian beam with nearspherical particle: an analytic-numerical approach for assessing scattering and stresses. Journal of the Optical Society of America A, Optics and Image Science 26, 1814-1826 (2009).

228. Boyde, L., Ekpenyong, A., Whyte, G. \& Guck, J. Comparison of stresses on homogeneous spheroids in the optical stretcher computed with geometrical optics and generalized Lorenz-Mie theory. Applied Optics 51, 7934-7944 (2012).

229. Lincoln, B., Schinkinger, S., Travis, K., Wottawah, F., Ebert, S., Sauer, F. \& Guck, J. Reconfigurable microfluidic integration of a dual-beam laser trap with biomedical applications. Biomedical Microdevices 9, 703-710 (2007).

230. Grosser, S., Fritsch, A. W., Kießling, T. R., Stange, R. \& Käs, J. A. The lensing effect of trapped particles in a dual-beam optical trap. Optics Express 23, 5221-5235 (2015).

231. Delabre, U., Feld, K., Crespo, E., Whyte, G., Sykes, C., Seifert, U. \& Guck, J. Deformation of phospholipid vesicles in an optical stretcher. Soft Matter 11, 6075-6088 (2015).

232. Solmaz, M. E., Biswas, R., Sankhagowit, S., Thompson, J. R., Mejia, C. A., Malmstadt, N. \& Povinelli, M. L. Optical stretching of giant unilamellar vesicles with an integrated dual-beam optical trap. Biomedical Optics Express 3, 2419-2427 (2012).

233. Guck, J., Ananthakrishnan, R., Mahmood, H., Moon, T. J., Cunningham, C. C. \& Käs, J. The Optical Stretcher: A Novel Laser Tool to Micromanipulate Cells. 81, 767-784 (2001).

234. Luke, D. R. Relaxed averaged alternating reflections for diffraction imaging. Inverse Problems 21, 37-50 (2005).

235. Gilbert, P. Iterative methods for the three-dimensional reconstruction of an object from projections. Journal of Theoretical Biology 36, 105-117 (1972).

236. Van Aarle, W., Palenstijn, W. J., De Beenhouwer, J., Altantzis, T., Bals, S., Batenburg, K. J. \& Sijbers, J. The ASTRA Toolbox: A platform for advanced algorithm development in electron tomography. Ultramicroscopy 157, 35-47 (2015). 
237. Van Aarle, W., Palenstijn, W. J., Cant, J., Janssens, E., Bleichrodt, F., Dabravolski, A., Beenhouwer, J. D., Batenburg, K. J. \& Sijbers, J. Fast and flexible X-ray tomography using the ASTRA toolbox. Optics Express 24, 25129-25147 (2016).

238. Jiang, H. et al. Quantitative 3D imaging of whole, unstained cells by using Xray diffraction microscopy. Proceedings of the National Academy of Sciences 107, 11234-11239 (2010).

239. Giewekemeyer, K. et al. Tomography of a Cryo-immobilized Yeast Cell Using Ptychographic Coherent X-Ray Diffractive Imaging. Biophysical Journal 109, 1986-1995 (2015).

240. Liu, J., Lhermitte, J., Tian, Y., Zhang, Z., Yu, D. \& Yager, K. G. Healing X-ray scattering images. IUCrJ 4, 455-465 (2017). 


\section{Authors contribution}

Five peer-reviewed articles are considered for this cumulative thesis to fulfill the rules and regulations of the $\mathrm{PhD}$ program ProPhys that is part of the Georg-August University School of Science (GAUSS):

(1) J.-D. Nicolas, M. Bernhardt, A. Markus, F. Alves, M. Burghammer and T. Salditt, "Scanning X-ray diffraction on cardiac tissue: automatized data analysis and processing", Journal of Synchrotron Radiation 24, 1163 - 1172 (2017). doi:10.1107/S1600577517011936.

(2) J.-D. Nicolas, M. Bernhardt, M. Krenkel, C. Richter, S. Luther and T. Salditt, "Combined scanning X-ray diffraction and holographic imaging of cardiomyocytes", Journal of Applied Crystallography 50, 612 - 620 (2017). doi:10.1107/S1600576717003351.

(3) E. Carboni*, J.-D. Nicolas*, M. Töpperwien, C. Stadelmann-Nessler, P. Lingor and T. Salditt, "Imaging of neuronal tissues by x-ray diffraction and $\mathrm{x}$-ray fluorescence microscopy: evaluation of contrast and biomarkers for neurodegenerative diseases", Biomedical Optics Express 8, 4331 - 4347 (2017). doi:10.1364/BOE.8.004331.

(4) J.-D. Nicolas, J. Hagemann, M. Sprung and T. Salditt, "The optical stretcher as a tool for single particle X-ray imaging and diffraction", Journal of Synchrotron Radiation 25, 1196 - 1205 (2018). doi:10.1107/S1600577518006574.

(5) J.-D. Nicolas, M. Bernhardt, S. F. Schlick, M. Tiburcy, W.-H. Zimmermann, A. Khan, A. Markus, F. Alves, K. Toischer and T. Salditt, "X-ray diffraction imaging of cardiac cells and tissue", Progress in Biophysics and Molecular Biology, in press (2018). doi:10.1016/j.pbiomolbio.2018.05.012.

* shared first authorship

Article (1) describes the analysis tools developed by JDN and made them publicly available. The analysis routines have been written by JDN, the experiment was performed by JDN with assistance by $\mathrm{MBu}$. The sample was provided by AM and FA. The results were discussed by all authors. JDN and TS wrote the manuscript. In (2), a combination of X-ray holography with scanning X-ray scattering is described. JDN, MB and TS performed the experiment and JDN, MB 
further prepared the samples that have initially been provided by SL and CR. JDN analyzed the data. MK assisted in the analysis of the X-ray holography dataset. JDN and TS wrote the manuscript. Scanning X-ray diffraction was performed on neuronal tissue in (3). The tissue samples were prepared by PL, EC and CSN. JDN, EC, MT, PL and TS performed the experiment. EC performed the histological examination. JDN and EC analyzed the X-ray fluorescence dataset. X-ray scattering data was analyzed by JDN. JDN, EC, PL and TS wrote the manuscript. Article (4) describes the integration of a commercially available optical stretcher into a synchrotron experiment. JDN prepared the experiment. JDN and TS performed the experiment with assistance by MS. JH provided the framework for the analysis of the X-ray dataset. JDN and JH analyzed the data. JDN and TS wrote the manuscript. The results presented in (5) were analyzed by JDN, KT provided adult cardiomyocytes. SS, MT and WHZ prepared human iPS cells derived into cardiomyocytes that were then further processed by JDN and MB. WHZ and MT provided engineered heart tissue that was further processed by JDN. AK, AM and FA provided heart tissue sections. JDN and TS wrote the manuscript. 


\section{Publications}

(1) J.-D. Nicolas, M. Bernhardt, A. Markus, F. Alves, M. Burghammer and T. Salditt, "Scanning X-ray diffraction on cardiac tissue: automatized data analysis and processing", Journal of Synchrotron Radiation 24, 1163 - 1172 (2017). doi:10.1107/S1600577517011936.

(2) J.-D. Nicolas, M. Bernhardt, M. Krenkel, C. Richter, S. Luther and T. Salditt, "Combined scanning X-ray diffraction and holographic imaging of cardiomyocytes", Journal of Applied Crystallography 50, 612 - 620 (2017). doi:10.1107/S1600576717003351.

(3) J.-D. Nicolas, M. Bernhardt, S. F. Schlick, M. Tiburcy, W.-H. Zimmermann, A. Khan, A. Markus, F. Alves, K. Toischer and T. Salditt, "X-ray diffraction imaging of cardiac cells and tissue", Progress in Biophysics and Molecular Biology, in press (2018). doi:10.1016/j.pbiomolbio.2018.05.012.

(4) E. Carboni*, J.-D. Nicolas* ${ }^{*}$ M. Töpperwien, C. Stadelmann-Nessler, P. Lingor and T. Salditt, "Imaging of neuronal tissues by x-ray diffraction and $\mathrm{x}$-ray fluorescence microscopy: evaluation of contrast and biomarkers for neurodegenerative diseases", Biomedical Optics Express 8, 4331 - 4347 (2017). doi:10.1364/BOE.8.004331.

(5) J.-D. Nicolas, J. Hagemann, M. Sprung and T. Salditt, "The optical stretcher as a tool for single particle X-ray imaging and diffraction", Journal of Synchrotron Radiation 25, 1196 - 1205 (2018). doi:10.1107/S1600577518006574.

(6) M. Reichardt, J. Frohn, M. Töpperwien, J.-D. Nicolas, A. Markus, F. Alves, and T. Salditt, "Nanoscale holographic tomography of heart tissue with $\mathrm{x}$ ray waveguide optics", Proc. of SPIE 10391, 1039105 (2018).

doi: $10.1117 / 12.2276648$.

(7) A.D. Surowka, M. Töpperwien, M. Bernhardt, J.-D. Nicolas, M. Osterhoff, T. Salditt, D. Adamek and M. Szczerbowska-Boruchowska, "Combined insitu imaging of structural organization and elemental composition of substantia nigra neurons in the elderly", Talanta 161, 368-376 (2016). doi:10.1016/j.talanta.2016.08.023.

(8) M. Bernhardt, J.-D. Nicolas, M. Eckermann, B. Eltzner, F. Rehfeldt and 
T. Salditt, "Anisotropic x-ray scattering and orientation fields in cardiac tissue cells", New Journal of Physics 19, 013012 (2017).

doi:10.1088/1367-2630/19/1/013012.

(9) D. Gur, J.-D. Nicolas, V. Brumfeld, O. Bar-Elli, D. Oron, G. Levkowitz, "The Dual Functional Reflecting Iris of the Zebrafish", Advanced Science 5, 1800338 (2018). doi:10.1002/advs.201800338.

(10) M. Bernhardt, J.-D. Nicolas, M. Osterhoff, H. Mittelstädt, M. Reuss, B. Harke, A. Wittmeier, M. Sprung, S. Köster and T. Salditt, "Correlative microscopy approach for biology using X-ray holography, X-ray scanning diffraction and STED microscopy", Nature Communications 9, 3641 (2018). doi:10.1038/s41467-018-05885-z.

* shared first authorship 


\section{Acknowledgements}

This work would not have been possible without intensive collaboration. Collaborations occurred on all length scales: within the Institute for X-ray Physics (IRP), between groups from the University of Göttingen and also with institutes inside and outside of Germany. However, foremost I would like to thank Prof. Dr. Tim Salditt for his inspiration, constant guidance and motivational speeches. You, Tim, have clearly been the inspirational powerhouse for this work and I am very happy that I could work with you throughout many past years!

I also thank Prof. Dr. Eberhardt Bodenschatz for co-supervising my thesis and Prof. Dr. Sarah Köster, Prof. Dr. Wolfram-Hubertus Zimmermann, Prof. Dr. Florian Rehfeldt and PD Dr. Martin Wenderoth for refereeing this thesis.

Within the institute I am most indebted to Marten Bernhardt who I could follow up on this project and who always offered his support, who joined me on many beamtimes and with whom I could enjoy many insightful conversations. Thank you for always keeping your door open for me. The same can be said about Johannes Hagemann and Aike Ruhlandt who have become two invaluable friends throughout the years at the IRP. Much office time was also shared with Sebastian Aeffner and I am very glad that we can finally work together on our mutual interest of radiation damage. Much of this work would also not have been possible without the help by Markus Osterhoff, who implemented the continuous scanning modality at beamline P10 alongside many other things that made me and the live of many other members of the IRP easier. The BioBio-team Susanne Hengst and Kristin Müller dedicated much of their time to prepare samples for and even during beamtimes. I am grateful for assistance in my work on holographic imaging and tomography by Martin Krenkel, Mareike Töpperwien, Malte Vassholz and Marina Eckermann. I thank you and all other members and alumni of the IRP for providing such a good work environment!

During my doctoral studies we also reached out for assistance by other groups at the Göttingen Campus and I am very happy that our call for collaboration was always met with great enthusiasm. From the group of Stefan Luther, Tina Althaus, Marion Kunze and Claudia Richter provided us with cardiomyocytes. When the 
work started to include structural analysis of tissue, we found in the group of Frauke Alves an invaluable partner. Frauke Alves, Andrea Markus and Amara Khan have supported us in many ways and I was always very happy to meet and discuss with them. The collaboration with Wolfram-Hubertus Zimmermann and his team offered new and exciting possiblities. I thank Malte Tiburcy for his help in the organization of our collaboration and for providing the engineered heart muscle samples. The iPS cells were prepared by Susanne Schlick who has always been enviably motivated and helpful! Our most recent collaboration was a small landmark for this project and I am very glad we got into contact with Dr. Karl Toischer to receive adult cardiomyocytes that have proved a valuable sample for us. The isolation of the cells was performed by Alessya Jacqueline Kretzschmar. I also thank Wiebke Möbius and Torben Ruhwedel for helpful discussions, their support in isolating optical nerve fibers and in supplying Karlsson-Schulz fixation solution.

I thank Michael Sprung and Eric Stellamanns from beamline P10, DESY for providing such a wonderful experimental endstation and their corresponding laboratories. I was greatly impressed by the trimmed state of all of their equipment. The optical stretcher experiment that was conducted at this beamline would not have been possible without the generous borrowing of the optical stretcher and further help and guidance in the configuration and operation of the setup given by Roland Stange.

As I spent many months of my doctoral studies at the ESRF in Grenoble, France, I have made many contacts that I would like thank at this point for their motivational support and scientific influence. First, I would like to thank Diego Pontoni for making our collaboration with the ESRF through the Partnership for Soft Condensed Matter (PSCM) possible. He always offered the assistance of the PSCM during beamtimes and even for building a cryogenic chamber. Scientifically, this project was motivated by our need for cryogenic protection of cells during scanning but much of was a rather coarse idea was made more concrete by the help of Martin Rosenthal from beamline ID13 who even had the idea of combining AFM scanning with a cryogenic chamber. As the project converged, all the expertise in the design of the final product came from Peter van der Linden from the PSCM without whom none of the chamber would at any point have been built!

I am also very much indebted to Manfred Burghammer from ID13 for his fantastic enthousiasm and a support that I could have never asked for. I thank all other members of ID13, especially Anastasya Shilova, Andreas Walter Johannes, Tilman Grünewald and Britta Weinhausen for making me feel highly welcomed at the 
ESRF. I thank Felix Roosen-Runge for many inspiring conversations. I am most honored to have shared my ESRF office with Pierre Lloria, Victoria Kabanova and Alexandre Even. Vous avez été si indescriptiblement accueillant et je suis extrêmement chanceux de vous avoir comme amis!

At last, I would like to thank Jana Utermöhlen, Helge Busmann, the Göttingen Lindy Hoppers and my family and friends who have supported me during the course of my studies.

Funding by the Sonderforschungsbereich 937 "Collective behavior of soft and biological matter" (project A11) and the cluster of excellence 171 "Nanoscale Microscopy and Molecular Physiology of the Brain" by the German science foundation (DFG) as well as through grant O5K16M62 by the Federal Ministry of Education and Research (BMBF) is greatfully acknowledged. 



\section{Curriculum Vitae}

\section{Personal Information}

Jan-David Nicolas

Born: $\quad$ 17. August 1988 in Bad Pyrmont

Nationality: German

\section{Studies}

since $03 / 2015$

Doctoral studies in the group of Prof. Tim Salditt at the Institute for X-Ray Physics of Göttingen University, Germany, with intermittent stays at the European Synchrotron Radiation Facility (ESRF), France

$09 / 2014-03 / 2015$

Teaching and administration at the Tadlo Computer Education Center in Lao PDR as a scholar of the ASA-Program by Engagement Global gGmbH - Service für Entwicklungsinitiativen, Germany

$03 / 2014-08 / 2014$

Research assistant at the Institute for X-Ray Physics of Göttingen University, Germany

$02 / 2014$

Master's thesis: Time-resolved coherent diffractive imaging on surface acoustic waves

$10 / 2012-02 / 2014$

Master of science in physics with major in biophysics and complex systems at Göttingen University, Germany 
$06 / 2012-10 / 2012$

Internship at Philips Research Eindhoven, Netherlands

$08 / 2011-06 / 2012$

Exchange studies at Stockholm University, Sweden

$09 / 2011$

Bachelor's thesis: Phase-contrast imaging of black lipid membranes

$07 / 2010-10 / 2010$

Internship at the National Institute of Standards and Technology in Gaithersburg, MD, USA as scholar of the German Academic Exchange Service

$10 / 2008-09 / 2011$

Bachelor of science in physics at Göttingen University, Germany

$06 / 2008$

Abitur (general qualification for university entrance)

Staatliches Landschulheim Marquartstein, Germany 